Florida International University FIU Digital Commons

3-29-2010

\title{
Tailoring Heme-Thiolate Proteins into Efficient Biocatalysts with High Specificity and Selectivity
}

Hui Tian

Florida International University, htian001@fiu.edu

DOI: $10.25148 /$ etd.FI10041624

Follow this and additional works at: https://digitalcommons.fiu.edu/etd

\section{Recommended Citation}

Tian, Hui, "Tailoring Heme-Thiolate Proteins into Efficient Biocatalysts with High Specificity and Selectivity" (2010). FIU Electronic Theses and Dissertations. 172.

https://digitalcommons.fiu.edu/etd/172

This work is brought to you for free and open access by the University Graduate School at FIU Digital Commons. It has been accepted for inclusion in FIU Electronic Theses and Dissertations by an authorized administrator of FIU Digital Commons. For more information, please contact dcc@fiu.edu. 


\section{FLORIDA INTERNATIONAL UNIVERSITY}

Miami, Florida

TAILORING HEME-THIOLATE PROTEINS INTO EFFICIENT BIOCATALYSTS WITH HIGH SPECIFICITY AND SELECTIVITY

A dissertation submitted in partial fulfillment of the requirements for the degree of DOCTOR OF PHILOSOPHY

in

CHEMISTRY

by

Hui Tian 
To: Dean Kenneth Furton

College of Arts and Sciences

This dissertation, written by Hui Tian, and entitled Tailoring Heme-Thiolate Proteins into Efficient Biocatalysts with High Specificity and Selectivity, having been approved in respect to style and intellectual content, is referred to you for judgment.

We have read this dissertation and recommend that it be approved.

John Landrum

David Chatfield

Fenfei Leng

Martin Tracey

Xiaotang Wang, Major Professor

Date of Defense: March 29, 2010

The dissertation of Hui Tian is approved.

\begin{tabular}{r}
$\begin{array}{r}\text { Dean Kenneth Furton } \\
\text { College of Arts and Sciences }\end{array}$ \\
\hline Interim Dean Kevin O'Shea \\
University Graduate School
\end{tabular}

Florida International University, 2010 
(C) Copyright 2010 by Hui Tian

All rights reserved. 


\section{DEDICATION}

I dedicate this dissertation to my husband Xiao and my parents. Without their understanding, patience, support, and most of all love, the completion of this work would not have been possible. 


\section{ACKNOWLEDGMENTS}

My graduate study and research at FIU in the past few years have been an amazing and rewarding experience in my life. I am indebted to so many people that have helped me to grow professionally and personally during the journey.

First of all, I wish to express my sincerest and deepest gratitude to my major professor, Dr. Xiaotang Wang, for his incredible guidance, support, patience and encouragement through the years of my graduate study. I thank him for having faith in me and providing me with such an excellent atmosphere for doing research. I cannot ask for a better mentor and cannot thank him enough for what he has done for me.

I would like to extend my appreciation to my committee members: Dr. Fenfei Leng who helped me at the very beginning of my research projects and gave me so many valuable pieces of advices and guidance; Dr. John Landrum who has guided and supported my research and study in a lot of ways; and Dr. David Chatfield and Dr. Tracey Martin who have given me so many critical comments and helpful suggestions.

Special thanks go to Dr. Andrew Terentis at Florida Atlantic University who helped me with the resonance Raman experiments for more than two years which are indispensable parts of this work. His knowledge and guidance have led me to a new technique area. Many thanks to Dr. Chenzhong Li for his support and help with the electrochemical studies, and valuable suggestions with my original proposal. 
I would like to extend my heartfelt appreciation to Dr. Stanislaw Wnuk for all the care, support and encouragement he has given me along the years.

Special thanks must go to Yali HSu for the help with operation and maintenance of CD and NMR instruments, and Dr. Yucheng Jiang, Dr Yazhong Xiao, and Dr. Hedong Bian, the visiting professors in our department, for their kind assistance and inspiration to my research projects.

I thank all members of the Wang lab for their support, help, advice and friendship. I am really happy and lucky to spend the years with them who I have learned a lot from. I extend my thanks to all my friends in the chemistry department, biology department and biomedical engineering department for their generous help and encouragement, especially Shradha Prabhulkar for her kind help with the electrochemistry experiments.

I would like to acknowledge the organizations that have helped fund my research: National Science Foundation, Department of Chemistry \& Biochemistry and University Graduate School at Florida International University for the financial support of a Dissertation Evidence Acquisition Fellowship. Last, I offer my regards and gratitude to all of those who have supported me in any respect during the completion of this dissertation. 
ABSTRACT OF THE DISSERTATION

TAILORING HEME-THIOLATE PROTEINS INTO EFFICIENT BIOCATALYSTS

WITH HIGH SPECIFICITY AND SELECTIVITY

by

Hui Tian

Florida International University, 2010

Miami, Florida

Professor Xiaotang Wang, Major Professor

Cytochrome P450 monooxygenases, one of the most important classes of heme-thiolate proteins, have attracted considerable interest in the biochemical community because of its catalytic versatility, substrate diversity and great number in the superfamily. Although P450s are capable of catalyzing numerous difficult oxidation reactions, the relatively low stability, low turnover rates and the need of electron-donating cofactors have limited their practical biotechnological and pharmaceutical applications as isolated enzymes. The goal of this study is to tailor such heme-thiolate proteins into efficient biocatalysts with high specificity and selectivity by protein engineering and to better understand the structure-function relationship in cytochromes P450.

In the effort to engineer P450cam, the prototype member of the P450 superfamily, into an efficient peroxygenase that utilizes hydrogen peroxide via the "peroxide-shunt" pathway, site-directed mutagenesis has been used to elucidate the critical roles of 
hydrophobic residues in the active site. Various biophysical, biochemical and spectroscopic techniques have been utilized to investigate the wild-type and mutant proteins. Three important P450cam variants were obtained showing distinct structural and functional features. In P450camV247H mutant, which exhibited almost identical spectral properties with the wild-type, it is demonstrated that a single amino acid switch turned the monooxygenase into an efficient preoxidase by increasing the peroxidase activity nearly one thousand folds. In order to tune the distal pocket of P450cam with polar residues, Leu 246 was replaced with a basic residue, lysine, resulting in a mutant with spectral features identical to $\mathrm{P} 420$, the inactive species of $\mathrm{P} 450$. But this inactive-species-like mutant showed catalytic activities without the facilitation of any cofactors. By substituting Gly 248 with a histidine, a novel Cys-Fe-His ligation set was obtained in P450cam which represented the very rare case of His ligation in heme-thiolate proteins. In addition to serving as a convenient model for hemoprotein structural studies, the G248H mutant also provided evidence about the nature of the axial ligand in cytochrome P420 and other engineered hemoproteins with thiolate ligations. Furthermore, attempts have been made to replace the proximal ligand in sperm whale myoglobin to construct a heme-thiolate protein model by mimicking the protein environment of cytochrome P450cam and chloroperoxidase. 
I LITERATURE REVIEW .1

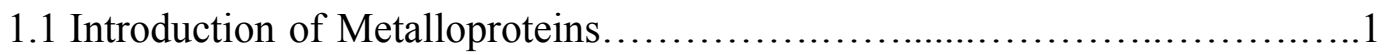

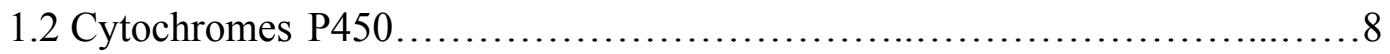

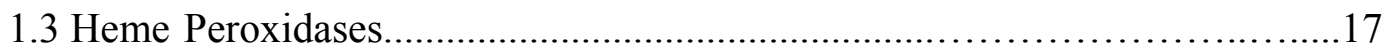

1.4 Protein Engineering of P450 and Heme Peroxidases ..........................24

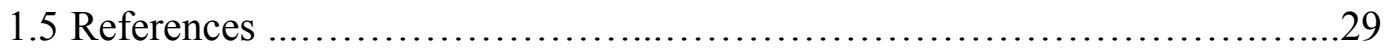

II SINGLE AMINO ACID SWITHCH TURNS P450CAM FROM A MONOOXYGENASE INTO AN EFFICIENT PEROXIDASE..................42

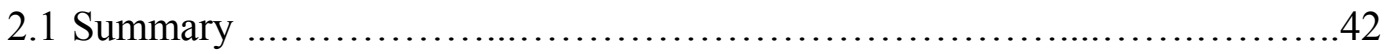

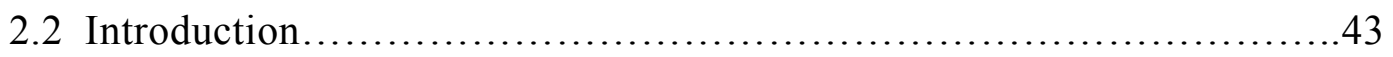

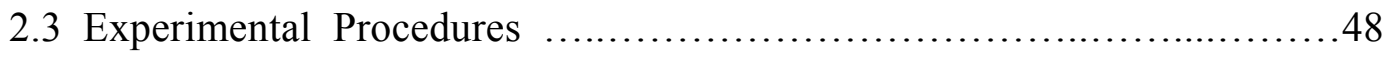

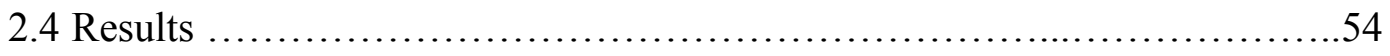

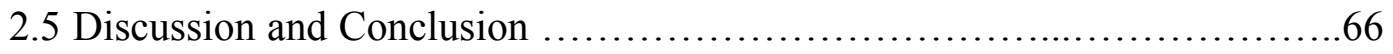

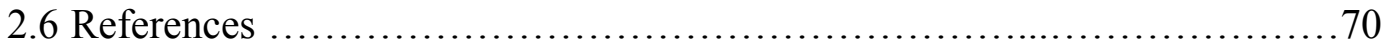

III ENGINEERING CYTOCHROME P450 INTO P420 WITH CATALYTIC

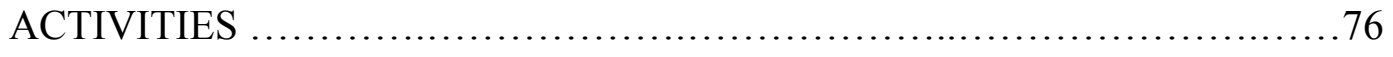

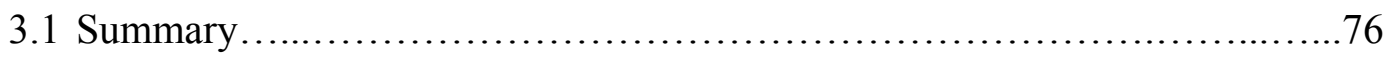

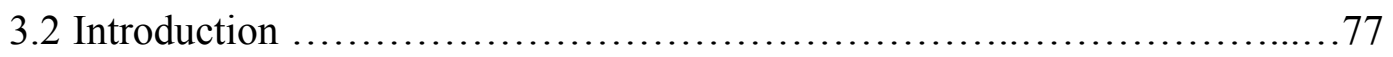

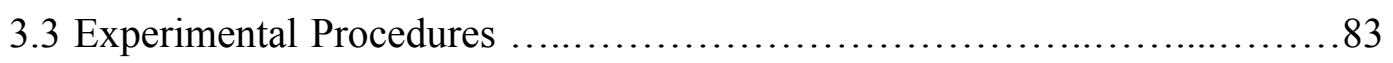

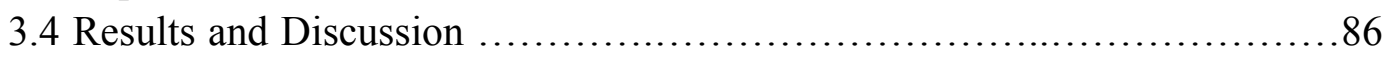

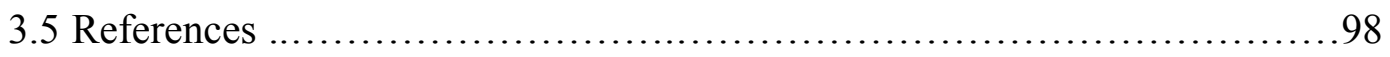

IV SPECTROSCOPIC STUDY OF A NOVEL HEME IRON LIGAND SET IN

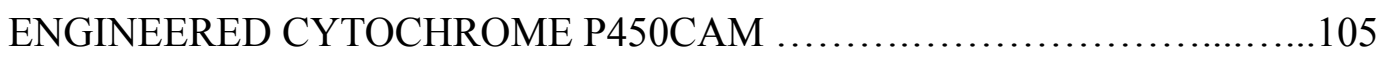

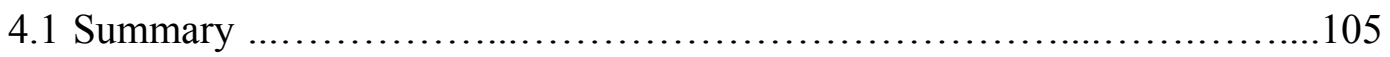

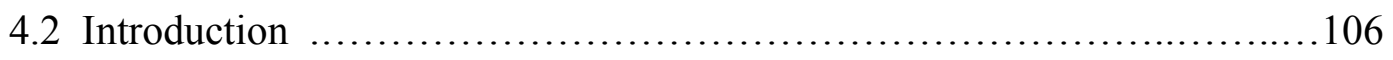

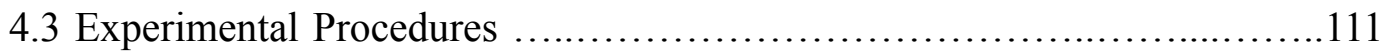

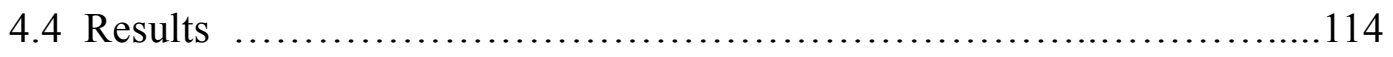

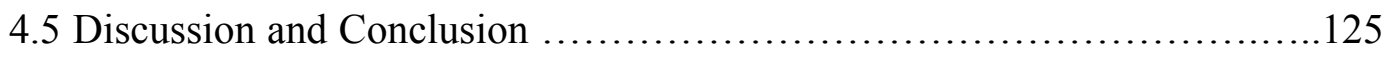

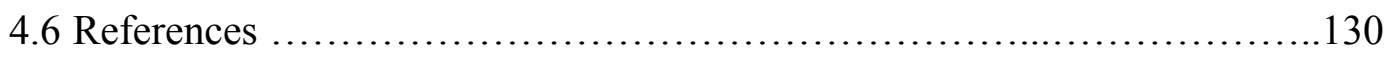

V PROTEIN ENGINEERING OF MYOGLOBIN WITH THIOLATE

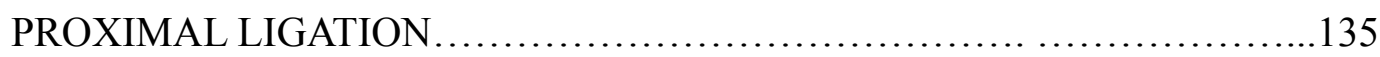

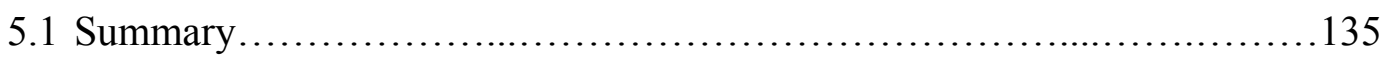

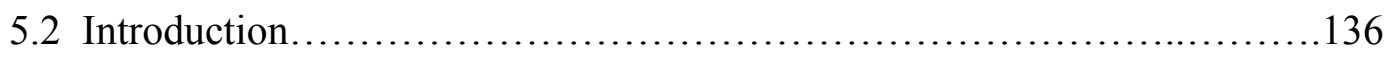


5.3 Experimental Procedures ................................................. 142

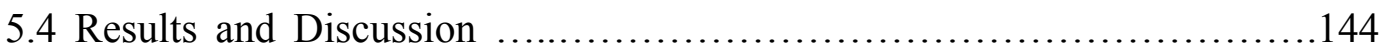

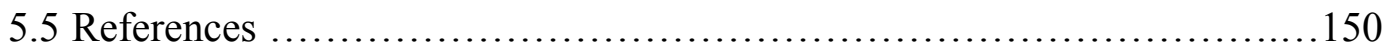

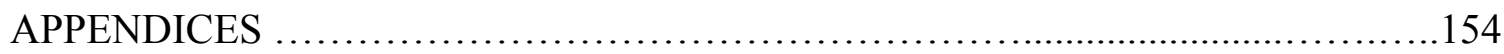

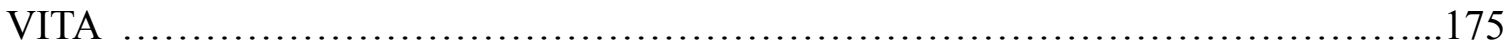




\section{LIST OF TABLES}

TABLE

PAGE

1.1 Heme-thiolate proteins and heme-thiolate protein mimics

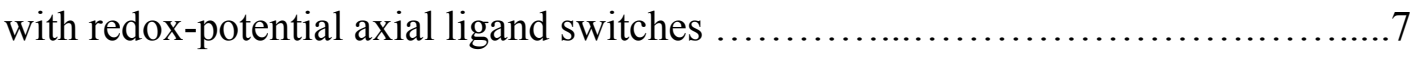

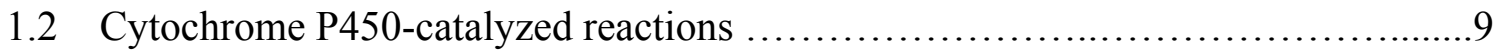

2.1 Wavelength and extinction coefficients of absorption maxima for wild type and $\mathrm{V} 247 \mathrm{H}$ mutant of P450cam ..................................56

3.1 Wavelength and extinction coefficients of absorption maxima for P450cam, P420cam, L246K and L246K 3H mutants

4.1 Wavelength and extinction coefficients of absorption maxima for wild type and $\mathrm{G} 248 \mathrm{H}$ mutant of P450cam .................................... 117

5.1 Proximal sequence alignment of $\mathrm{Mb}$ and its $\mathrm{P} 450$ and $\mathrm{CPO}$ mimics 142

5.2 Wavelength and extinction coefficients of absorption maxima for P450cam, Mb, MbP, and MbP H64G 


\section{LIST OF FIGURES}

FIGURE

PAGE

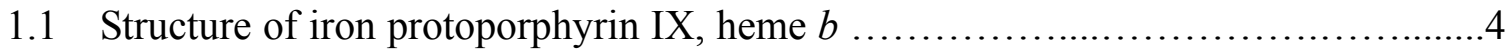

1.2 Heme centers of heme- thiolate proteins and

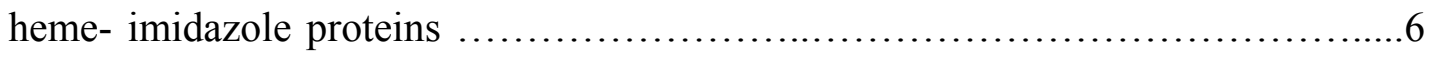

1.3 The cytochrome P450 catalytic cycle including the "peroxide-shunt" pathway

1.4 Cytochrome P450cam putative oxygen complex, Compound I ..................13

1.5 Crystal structure of cytochrome P450cam ................................... 16

1.6 Mechanism of Compound I formation in

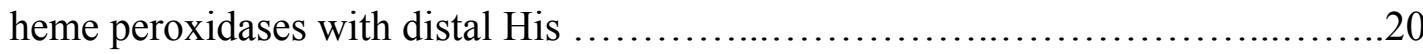

1.7 "Push-pull" mechanisms for O-O bond cleavage of both heme-imidazole proteins and heme-thiolate proteins.

1.8 Proposed mechanism of Compound I formation catalyzed by chloroperoxidase .23

2.1 The active site structure of camphor-bound cytochrome P450cam (V247)........47

2.2 UV-vis spectra of P450camV247H in various oxidation states ..................55

2.3 UV spectra of P450camV247H and its KCN bound complex ..................57

2.4 Far-UV CD and visible CD spectra of substrate-bound forms of the WT and V247H mutant of P450cam

2.5 Resonance Raman spectra of ferric and ferrous WT P450cam and $\mathrm{V} 247 \mathrm{H}$ mutant with and without camphor binding

$2.6 \quad 600-\mathrm{MHz}{ }^{1} \mathrm{H}$ Nuclear Magnetic Resonance spectra of the low spin cyanide complexes of WT P450 and V247H mutant

2.7 Cyclic voltammograms of WT P450cam and V247H mutant in the presence and absence of substrate camphor 
2.8 ABTS assay of WT P450cam, V247H and CPO at different $\mathrm{pH}$ values

3.1 Heme environmental structure of ferric cytochrome P450cam and chloroperoxidase

3.2 UV-visible spectra of $\mathrm{P} 450 \mathrm{H}$ in various oxidation states

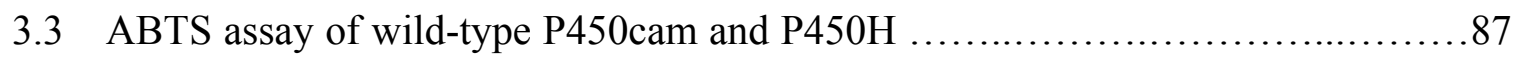

3.4 UV-visible spectra of P450camL246K in various oxidation states

3.5 Resonance Raman spectra of ferric and ferrous WT P450cam (with and without camphor), P420, L246K, L246K 3H mutants

3.6 Resonance Raman spectra of $\mathrm{Fe}^{\mathrm{II}}-\mathrm{CO}$ complexes of $\mathrm{P} 450$ cam L246K and L246K $3 \mathrm{H}$ mutants in low and high frequency regions

3.7 $\mathrm{Fe}^{2+}-\mathrm{CO}-\mathrm{Fe}^{2+}-{ }^{13} \mathrm{C}^{18} \mathrm{O}$ difference spectra of $\mathrm{P} 450$ camL246K in both low and high frequency regions

3.8 Correlation of $\mathrm{Fe}-\mathrm{CO}$ and $\mathrm{C}-\mathrm{O}$ stretching frequencies for different heme proteins and porphyrin derivatives (P450L246K)

3.9 ABTS assay of P450camL246K, P420 and CPO

4.1 The active site structure of camphor-bound cytochrome P450cam (G248)......110

4.2 UV-vis spectra of P450camG248H in various oxidation states ..................115

4.3 Resonance Raman spectra of ferric and ferrous WT P450cam and $\mathrm{G} 248 \mathrm{H}$ mutant with and without camphor binding

4.4 Resonance Raman spectra of ferrous-CO complexes of WT $\mathrm{P} 450$ cam and $\mathrm{G} 248 \mathrm{H}$ mutant in both low and high frequency

4.5 CD spectra of both camphor-free and camphor-bound forms of the WT and $\mathrm{G} 248 \mathrm{H}$ mutant of P450cam

4.6 Cyclic voltammograms of P450cam and G248H .124

5.1 A ribbon diagram of sperm whale myoglobin crystal structure 140

5.2 Crystal structure of sperm whale myoglobin active site .141 
5.3 UV spectra of $\mathrm{Mb}$ and $\mathrm{P} 450$ cam in various oxidation states

5.4 UV spectra of MbP in various oxidation states ..............................147

5.5 UV spectra of MbP H64G in various oxidation states ....................... 148 


\section{LIST OF ABBREVIATIONS}

ACRONYM

CYP

$\mathrm{CPO}$

HRP

CIP

CBS

$\mathrm{CcP}$

NOS

iNOS

CooA

$\mathrm{Pdx}$

Cpd 0

Cpd I

Cpd II

WT

$\mathrm{CO}$

SDS

PAGE

ITPG
FULL NAME

cytochromes P450

chloroperoxidase

horseradish peroxidase

Coprinus cinereus peroxidase

cystathionine $\beta$-synthase

cytochrome c peroxidase

nitric oxide synthase

inducible nitric oxide synthase

CO-sensing transcriptional activator

Putidaredoxin

compound 0

compound I

compound II

wild type

carbon monoxide

sodium dodecyl sulfate

polyacrylamide gel electrophoresis

isopropyl $\beta$-D-1-thiogalactopyranoside 


\begin{tabular}{|c|c|}
\hline PMSF & phenylmethylsulfonyl fluoride \\
\hline DEAE & diethylaminoethyl (cellulose) \\
\hline DTT & dithiothreitol \\
\hline NMR & nuclear magnetic resonance \\
\hline RR & resonance Raman \\
\hline $\mathrm{NAD}(\mathrm{P}) \mathrm{H}$ & nicotinamide adenine dinucleotide (phosphate), \\
\hline & reduced \\
\hline NHE & normal hydrogen electrode \\
\hline Tris & Tris-(hydroxymethyl)-aminomethane \\
\hline SWCNT & single-walled carbon nanotubes \\
\hline $\mathrm{Mb}$ & myoglobin \\
\hline $\mathrm{MbP}$ & myoglobin P450cam mimic \\
\hline $\mathrm{MbO}$ & myoglobin CPO mimic \\
\hline $\mathrm{Hb}$ & hemoglobin \\
\hline Ala/A & alanine \\
\hline Arg/R & arginine \\
\hline Asp/D & aspartic acid \\
\hline $\mathrm{Cys} / \mathrm{C}$ & cysteine \\
\hline Glu/E & glutamic acid \\
\hline Gly/G & glycine \\
\hline
\end{tabular}


His/H

Leu/L

Lys/K

Ser/S

$\mathrm{Thr} / \mathrm{T}$

$\mathrm{Val} / \mathrm{V}$ histidine

leucine

lysine

serine

threonine

valine 



\section{CHAPTER I. LITERATURE REVIEW}

\subsection{INTRODUCTION OF METALLOPROTEINS}

It is an old wisdom that metals are indispensable for life. Nature uses metal ions extensively for both structural and functional roles. By incorporating metal ions into proteins, a diverse class of biologically significant macromolecules termed metalloproteins is created. In fact, about one third to one half of all proteins require metals to carry out their functions (1). Metalloproteins can catalyze some of the most difficult and yet important transformations in nature, such as photosynthesis, respiration, electron transfer, water oxidation, molecular oxygen transport and reduction, and nitrogen fixation (2). The significance of iron, copper, and zinc in biological systems together with other metals including cobalt, manganese, molybdenum, and nickel has been evident for decades (3). The catalytic activities of these metal-containing systems depend on both the nature of the metal and the influence of the protein environment including the type of ligands provided by the protein, the geometry of the coordination sphere and the protein structure in the vicinity of the metal ion.

A great deal of effort has been devoted to the understanding of the structure and function of metalloproteins because of their indispensable roles in biological systems and their ability to catalyze some of the most difficult chemical reactions under mild and environmentally friendly conditions. Furthermore, such enzymes are able to catalyze 
regio- and stereo-selective transformations and thus offer products that are critical to the producrion of fine chemicals, pharmaceuticals, and many other value-added materials. To better understand the critical roles of metalloproteins, protein engineering has been used to study their structure-function relationships by either rational design or directed evolution.

\subsubsection{Heme-containing proteins}

Heme is comprised of a tetrapyrrole ring system complexed with an iron atom in the center. One or two axial ligands complete the coordination requirement of the heme iron. A heme protein (also known as hemoprotein or haemoprotein) contains a heme prosthetic group, bound either covalently or non-covalently to the protein. As one of the largest classes of metalloproteins, the heme-containing proteins have attracted a great deal of attention from the scientific community since the beginning of modern enzymology. They have diverse biological functions and are able to catalyze a broad spectrum of biological processes ranging from electron transfer (cytochromes) to oxygen storage (myoglobin) / transport (hemoglobin) / activation (P450) and molecular sensing (CooA) $(4-6)$.

Many biochemical and biophysical studies of heme proteins and their variants have suggested that the type of reaction catalyzed by different hemoproteins is normally controlled by: 1) the nature of the proximal ligand and its surrounding residues, 2) the distal environment including conserved significant residues, and 3) the type of heme (7). 
The most common heme prosthetic group is iron protoporphyrin IX also known as heme $b$ (Fig. 1.1), which is the prosthetic group found in all the heme proteins discussed in this dissertation. The ferriprotoporphyrin IX is made up of four pyrrol rings joined by methine bridges with ferric iron in the center of the molecule replacing the two hydrogen atoms attached to pyrrol nitrogens. The remaining two axial ligands to the heme iron are located above (distal) and below (proximal) the porphyrin plane respectively. Different types of heme have different side chains. There are eight side chains in ferriprotoporphyrin IX (heme $b$ ): four methyl groups at positions $1,3,5$, and 8 , two vinyl groups at positions 2 and 4, and two propionate groups at positions 6 and 7. The carbon atoms of the methine bridges are symbolized as $\alpha, \beta, \gamma$, and $\delta(8)$. In many heme proteins, oxygen or hydrogen peroxide will bind to the heme iron in the distal site as the proximal site is normally occupied by certain amino acid heteroatoms. For example, in most heme peroxidases, there is a histidine nitrogen serving as the proximal ligand; while chloroperoxidase, as an exception, possesses a cysteinate sulfur ligated to the heme iron. The distal side in these cases is the catalytic center, and its coordination state varies in different states of the enzyme during catalytic cycles. In some other heme proteins with no directly bound oxygen or hydrogen peroxide, both of the axial coordination sites are occupied by heteroatoms from the nearby amino acids, like cytochrome $\mathrm{c}$ which has a histidine and methionine as the axial ligands (9). 


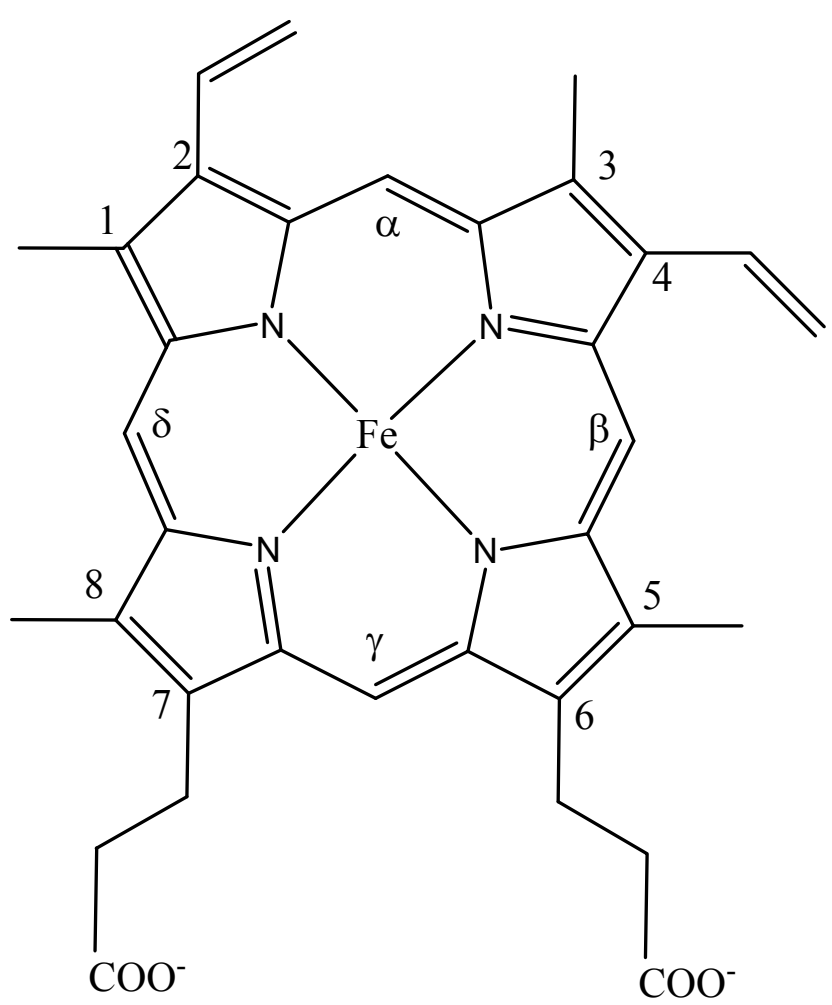

Figure 1.1 Structure of iron protoporphyrin IX, heme $b$. A-D indicate the location of each pyrrole ring and $\alpha$ to $\delta$ identify the site of each methane bridge.

One of the most important questions about heme proteins is how different heme proteins achieve such structural and functional diversity by using the same heme prosthetic group (5-7). For instance, hemoglobin and myoglobin are $\mathrm{O}_{2}$ carriers and can bind $\mathrm{O}_{2}$ reversibly $(10,11)$; heme oxygenase $(\mathrm{HO})$ and cytochromes $\mathrm{P} 450$ activate $\mathrm{O}_{2}$, and transfer the oxygen atom to the heme prosthetic group or to other organic molecules (12-15); FixL protein, CooA protein and guanylate cyclase serve as biological sensors for $\mathrm{O}_{2}, \mathrm{CO}$ and $\mathrm{NO}$ respectively (16). The protein environment, especially near the active site, clearly plays an essential role in governing the reactivity of the metal iron, and it could be the determining factor that controls the functionalities of the distinct hemoproteins with 
essentially identical metal centers. Therefore, the redesign of heme proteins, from one type into another, not only provides a test of the known factors governing their structure and function but also allows the direct comparison of two different heme proteins in the same framework to achieve protein variants with better efficiency and selectivity (7).

\subsubsection{Heme-Thiolate Proteins and their structural mimics}

Heme-thiolate proteins are a group of heme proteins with a thiolate anion from a deprotonated cysteine residue serving as the proximal heme ligand (Fig. 1.2 (A)). They constitute one of the two major categories of heme-containing proteins found in living organisms. The other group is the heme-imidazole proteins whose axial ligand is an imidazole group from a hisitidine residue (Fig. 1.2 B). Their difference in the axial ligand is also indicated in the optical properties of their reduced forms with carbon monoxide bound: the heme-thiolate proteins usually show a characteristic absorption maximum at around $450 \mathrm{~nm}$, whereas the corresponding absorption spectrum of heme-imidazole proteins give a Soret peak at around $420 \mathrm{~nm}$ (17). Therefore, the optical features of CO complexes have generally been used as a probe for the nature of proximal ligation in hemoproteins.

There are several classes of heme-thiolate proteins reported such as cytochromes P450, chloroperoxidase (CPO), nitric oxide synthases (NOS), cystathionine $\beta$-synthase, the sensor protein CooA and eIF2 $\alpha$ kinase (17). Heme-thiolate proteins are essentially among the most versatile biocatalysts of the hemoprotein family. They serve as 

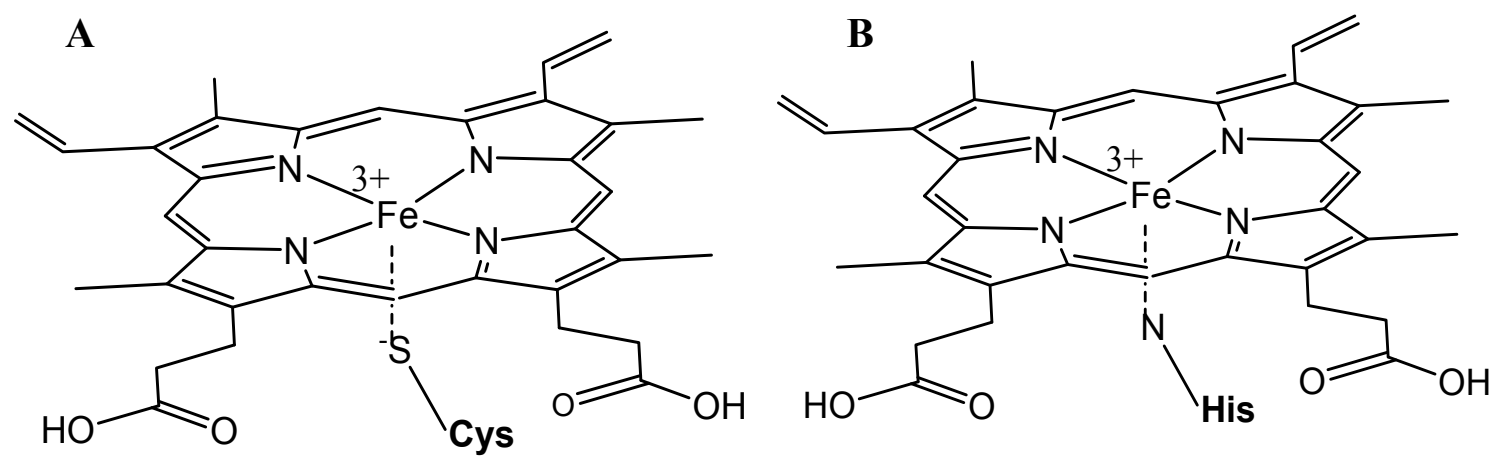

Figure 1.2 Heme centers of heme-thiolate proteins with Cys as the proximal ligand (A), and heme-imidazole proteins with His as the proximal ligand (B).

oxidoreductases that perform essential functions in the biological systems. The hallmark reaction catalyzed by these enzymes is the substrate oxidation, converting $\mathrm{R}-\mathrm{H}$ to $\mathrm{R}-\mathrm{OH}$ (18). It has been suggested that the identity of the axial ligands to the heme iron, the nature of the heme environment, and the accessibility of the substrate to the active site combine to play crucial roles in determining the enzyme activities. Because of the similarity of the protein environment that heme-thiolate proteins and heme-imidazole proteins share, many studies have been carried out to engineer the heme-imidazole proteins by replacing the proximal histidine with a cysteine residue to mimic heme-thiolate proteins, in order to better understand the structure-function relationships in these heme-containing enzymes.

In fact, the proximal thiolate ligation is not kept intact in various oxidation states in all heme-thiolate proteins, and in some cases the Fe-thiolate ligation is lost upon reduction and carbon monoxide binding. That also happens in many hemoproteins with 
engineered iron-thiolate ligands. Table 1.1 listed some examples of the heme-thiolate proteins and their mimics with redox-dependent axial ligands in various oxidation states.

Table 1.1. Heme-thiolate proteins and their mimics with redox-dependent axial ligand switches.

\begin{tabular}{lllll}
\hline Protein & Fe (III) & Fe (II) & Fe (II)-CO & Ref \\
\hline Heme-thiolate proteins & & & & \\
\hline P450 & Cys/OH & Cys & Cys/CO & $(13)$ \\
CPO & Cys/OH & Cys & Cys/CO & $(19-21)$ \\
NOS & Cys/OH & Cys & Cys/CO & $(22,23)$ \\
CooA & Cys/Pro & His/Pro & His/CO & $(24,25)$ \\
CBS & Cys/His & Cys/His & His/CO & $(26,27)$ \\
\hline Engineered heme-thiolate & protein mimics & & \\
\hline Mb H93C & Cys & His & His/CO & $(28,29)$ \\
CcP H175C/D235L & Cys & Cysteic acid & Cysteic acid/CO & $(30)$ \\
Cytochrome c M80C & Cys/His & His & His/CO & $(9)$ \\
Heme oxygenase H25C & Cys/H2O & & CO & $(31)$ \\
NP1 H60C & Cys & H $2 \mathrm{O}$ & CO & $(32)$ \\
Cytochrome $b 5$ H39C & Cys/His & His & His/CO & $(33)$ \\
\hline
\end{tabular}

For instance, in the CO-sensing transcriptional activator CooA, the Cys75 axial ligand of the ferric heme is exchanged to His77 upon reduction and $\mathrm{CO}$ activation. Therefore, the electronic absorption spectrum of CO-complex of CooA exhibits the Soret band at around $420 \mathrm{~nm}$ instead of $450 \mathrm{~nm}$, typical of thiolate-ligated hemoproteins (25). In the listed hemoproteins with engineered heme-thiolate ligations in their ferric forms (Table 1.1), upon reduction the iron-thiolate ligands are all lost either by oxidation, dissociation or protonation, resulting in ferrous-CO complexes with 420-nm Soret 
absorption maxima. Heme oxygenase uses His-25 as its proximal iron ligand. The substitution of His- 25 with a cysteine results in a 6-coordinated ligation of the iron to a cysteinate and water molecule, but the Fe-Cys bond is lost upon being reduced and the ferrous heme is only loosely bound to a postulated water molecule (31).

\section{2 CYTOCHROMES P450}

Cytochrome P450 (CYP) monooxygenase, a red pigment first found in liver microsomes and named for its spectral properties, is one of the most important heme-thiolate proteins. The ability of P450s to functionalize unactivated hydrocarbon at physiological conditions has attracted considerable interests from the chemical and biological communities (13). There are several books dedicated to the superfamily of P450 enzymes $(14,34-39)$. Many reviews have elaborated the chemistry, structure and functions of P450s $(13,40-44)$. The P450 enzymes have been identified in all domains of life and more than 11,500 distinct CYP proteins had been known and named as of Feb. 2009 (45). In all P450s, the heme-thiolate ligation is highly conserved in the proximal side of the active site and is considered to play critical roles in the catalytic mechanisms. The cytochromes P450 perform regio- and stereo-specific reactions under physiologial conditions and the most common reaction catalyzed by cytochrome P450 is a 
monooxygenase reaction in which one atom of oxygen is inserted into an organic substrate (RH) while the other oxygen atom is reduced to water (Eq. 1).

$$
\mathrm{R}-\mathrm{H}+\mathrm{NAD}(\mathrm{P}) \mathrm{H}+\mathrm{H}^{+}+\mathrm{O}_{2} \stackrel{\mathrm{P} 450}{\longrightarrow} \mathrm{R}-\mathrm{OH}+\mathrm{NAD}(\mathrm{P})^{+}+\mathrm{H}_{2} \mathrm{O}
$$

The P450s generally require two electrons from either $\mathrm{NADH}$ or $\mathrm{NAD}(\mathrm{P}) \mathrm{H}$ to activate $\mathrm{O}_{2}$, and the electrons are transferred to the heme of $\mathrm{P} 450$ via a protein electron transport system, usually a reductase and an iron-sulfur protein, depending on the type of P450s. In addition to hydroxylation, P450s are able to catalyze a number of other significant reactions as listed in Table 1.2. The catalytic versatility, substrate diversity and sheer number of P450s have lead to considerable interest over the last few decades in the engineering of P450 enzymes for a variety of purposes.

Table 1.2 Cytochrome P450-catalyzed reactions $(34,46)$.

C-H hydroxylation

Epoxidation

Allylic Rearrangement

Desaturation

Reductive Dehalogenation
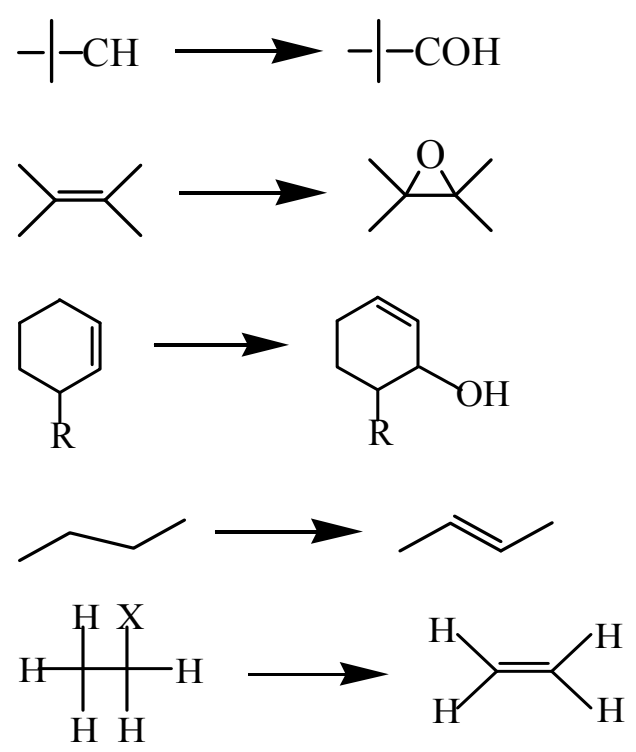
Oxidative Dehalogenation

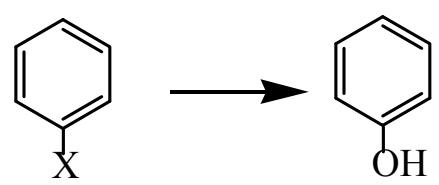

Alcohols oxidation<smiles>CC=CCCC=O</smiles>

Aldehyde oxidation

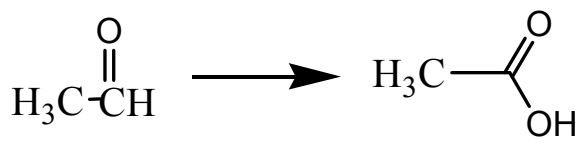

Deformylation

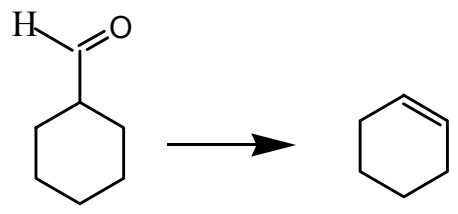

Oxidative deformylation

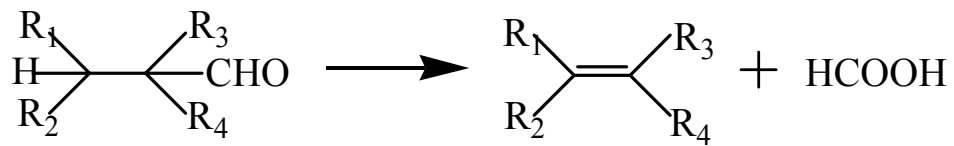

Sulfoxidation

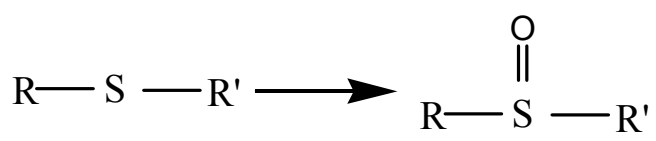

N-Dealkylation

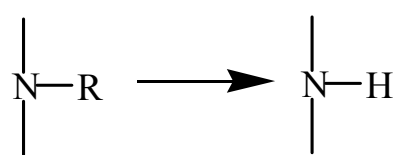

O-Dealkylation

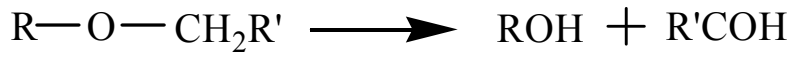

N-Oxygenation

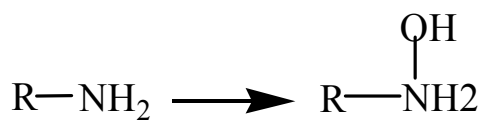

Peroxidase-type oxidation

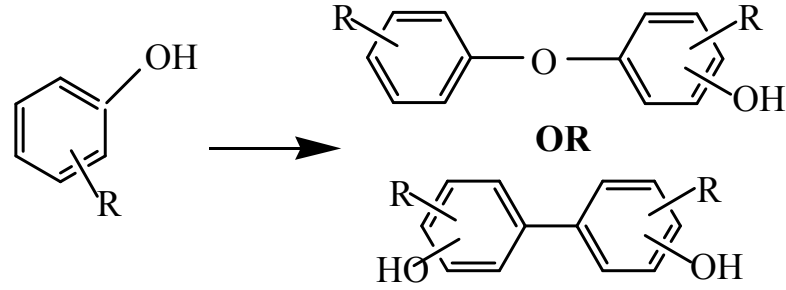

NO Synthase-type oxidation

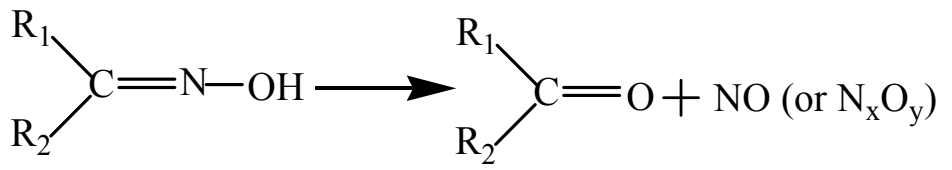




\subsubsection{Catalytic cycle of P450s}

The generally accepted catalytic cycle of P450 enzymes is shown in Fig. 1.3 (34). The resting state (1) is a six-coordinate, low-spin ferric heme, with poryphyrin nitrogen atoms occupying the four equatorial position and cysteine and water serving as the axial ligands at the proximal and distal sides, respectively. The catalytic cycle is initiated by the substrate binding in the protein active site followed by the displacement of the distal water molecule, converting the six-coordinate low-spin ferric heme to a predominantly five-coordinate high-spin form (intermediate (2)) (47). In the meantime, the reduction potential is increased by greater than $+100 \mathrm{mV}$ (substrate-dependent) upon substrate binding, which has been shown to initiate electron transfer to the heme from NAD(P)H via native reductase proteins (48). The first electron transfer leads to the reduction of ferric to ferrous heme (intermediate (3)), followed by dioxygen binding at the ferrous heme iron, yielding an unstable ferrous-oxy species (intermediate (4)). The second electron transfer is suggested to be the rate- limiting step in the cycle followed by protonation and the formation of the hydroperoxo-iron complex (intermediate (6), Compound 0). Then this species is further protonated to generate highly reactive Compound I (49), ferryl-oxo porphyrin $\pi$-radical cation, the key intermediate (7) in the catalysis (Fig. 1.4). This step involves the heterolytic splitting of the oxygen-oxygen bond and the releasing of a water molecule. A highly conserved threonine residue near the heme has been suggested to play an essential role to relay protons from the solvent to 
the heme $(50,51)$. Finally, the Fe-ligated oxygen atom is transferred to the substrate through intermediate (8) followed by hydroxylation and regeneration of the resting state.

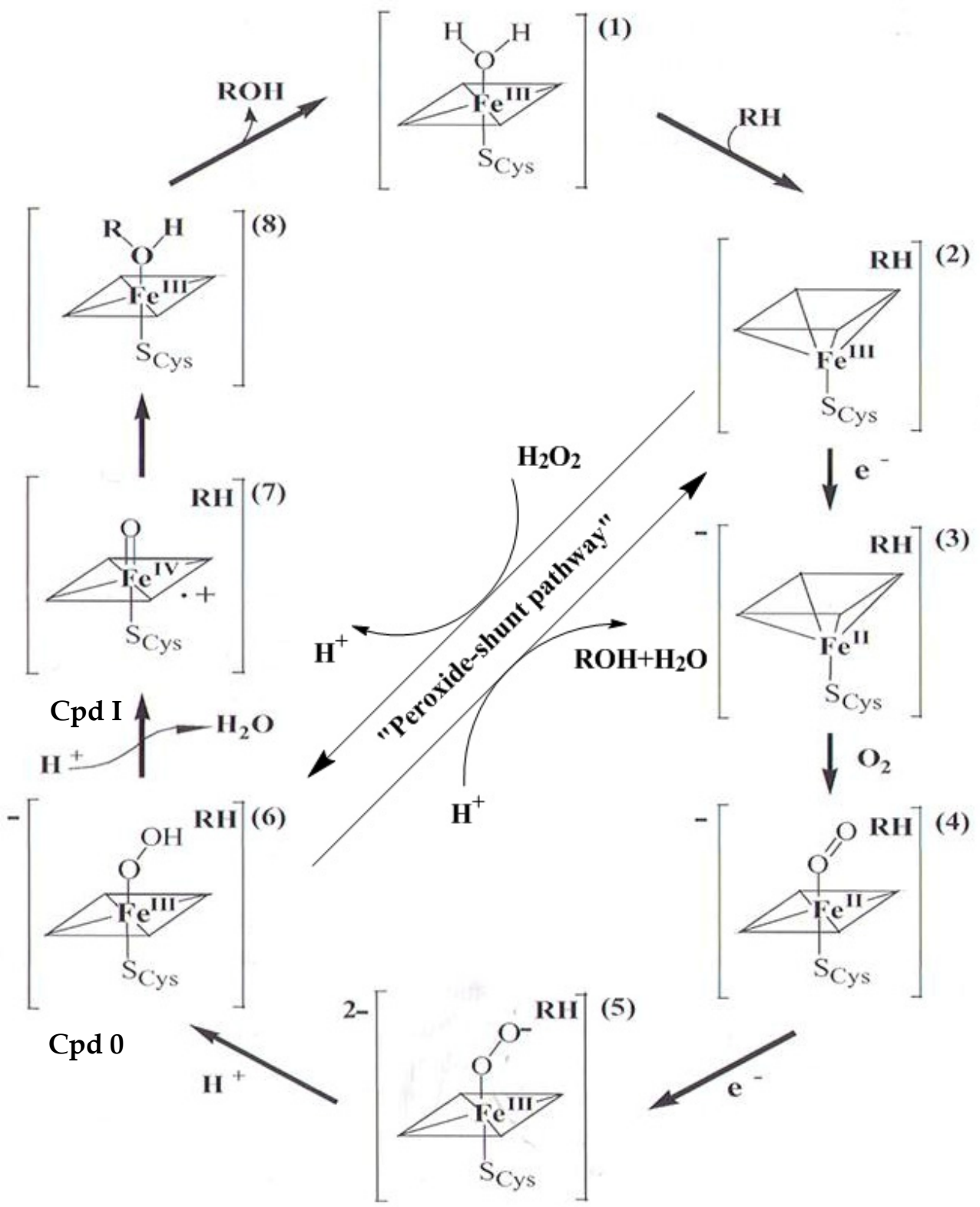

Figure 1.3 The cytochrome P450 catalytic cycle including the "peroxide-shunt pathway". RH is substrate and $\mathrm{ROH}$ is the product. The porphyrin molecule is represented as a parallelogram. Intermediates (6) and (7) are Compound 0 and Compound I respectively. 


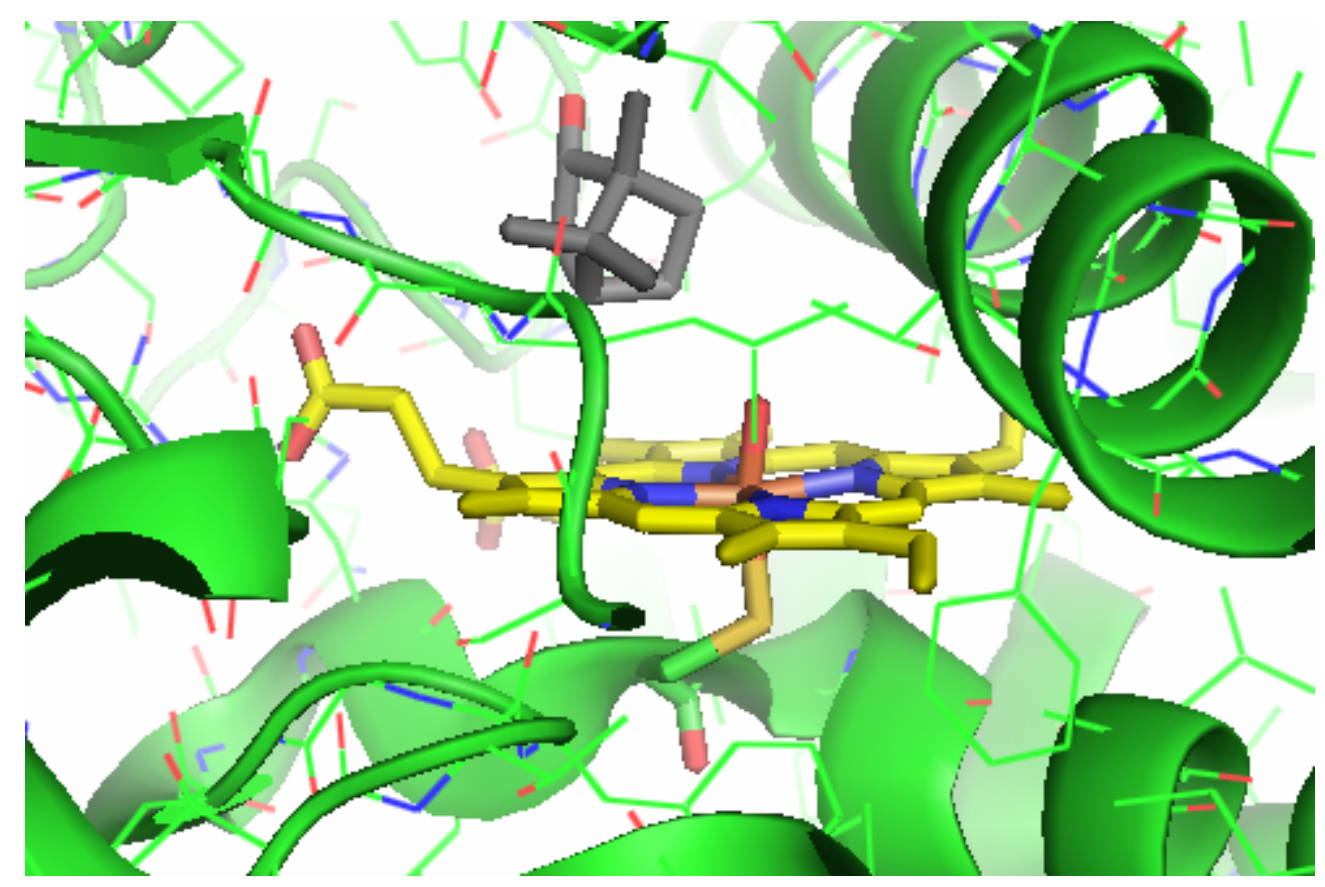

Figure 1.4 P450cam putative oxygen complex, Compound I. The heme prosthetic group is colored in yellow, the substrate is in gray and the bound dioxygen is in red. All other amino acids are depicted in lines and ribbons. This image was created from the crystal data (PDB code 1DZ9) using PYMOL (52).

In the standard catalytic cycle, Compound I would hydroxylate the substrate and regenerate the $\mathrm{P} 450$ resting state. Although the main steps mentioned above are similar for all P450 enzymes, each step is far from being simple and many side reactions exist leading to the leakage of the cycle (34). 


\subsection{2 "Peroxide-shunt" pathway}

In the absence of reducing cofactors, partner proteins and dioxygen, P450s are capable of utilizing hydrogen peroxide or organic peroxy compound as the oxidant to hydroxylate substrates through the "peroxide-shunt" pathway (Eq. 2) (14):

$$
\mathrm{RH}+\mathrm{R}^{\prime} \mathrm{OOH} \stackrel{\mathrm{P} 450}{\longrightarrow} \mathrm{ROH}+\mathrm{R}^{\prime} \mathrm{OH}
$$

As illustrated in Fig. 1.3, the shunt pathway bypasses a large portion of the standard catalytic cycle by eliminating the rate-limiting electron transfer steps and directly results in the generation of the $\mathrm{Fe}^{\mathrm{III}}-\mathrm{OOH}-$ complex (Cpd. 0). There are many studies about the P450 peroxide shunt pathway (53-59). This type of reaction is not suggested to be physiological, since the turnover rate is quite low and high concentrations of peroxide are needed to achieve the optimal reaction rate. But on the other hand, some unique P450 enzymes have been reported to function as peroxygenases by utilizing hydrogen peroxide or the peroxide groups of their subsbtrates as the oxygen donor with high catalytic turnover rates, compared with a normal monooxygenation reaction or the peroxide-shunt reaction catalyzed by common P450s (60-66). The "peroxygenase" activity of P450s has definitely broadened the versatility of the superfamily and offers a possibility to utilize cell-free P450-dependent catalysis without the requirement of $\mathrm{NAD}(\mathrm{P}) \mathrm{H}$, and auxiliary proteins. 


\subsubsection{P450 Structure}

In the massive superfamily of cytochrome P450s, there are more than 11,500 distinct CYP proteins known to date, while the sequence identities between the P450 families are generally low (about 10-30\%) (45, 67). Despite the sequence diversities, P450 in many different families and many different organisms share a high degree of structural conservation in their secondary and tertiary folds $(34,68,69)$. So far, there are over 300 unique P450 structures available in the Protein Data Bank. Most P450 enzymes are normally described as consisting of $\alpha$ helical and $\beta$ sheet domains. Unlike peroxidases which clearly fold into $\mathrm{N}$ - and C-terminal helical-rich domains with the heme sandwiched between the helices from each domain, P450s do not fold into distinctly divided $\mathrm{N}$ - and C-terminal domains. For example, cytochrome P450cam, the first protein to be crystallized in the P450 superfamily, divides into a helix-rich domain on the right side and a $\beta$-sheet-rich domain on the left side (Fig. 1.5). The heme group is bracketed between the proximal helix $\mathrm{L}$ and distal helix $\mathrm{I}$ in the $\mathrm{C}$ terminal half of the molecule, while a majority of the $\beta$-structure is also in the $\mathrm{C}$-terminal half but located far from heme $(70,71)$.

The heme prosthetic group is deeply embedded in the hydrophobic interior, which itself provides the largest hydrophobic surface for interactions with the substrates. The heme is oriented nearly parallel to the surfaces between helices L and I with no significant exposure to the protein surface (13). The most distinct structural feature of 
P450 is the presence of an unusual proximal ligation, thiolate sulfur provided by a deprotonated cysteine residue. It has been generally agreed that the Cys thiolate ligand to the heme iron and its surrounding protein environment play unique and critical roles in P450 catalysis. In P450s, the protein architecture surrounding the proximal Fe-Cys ligation is highly conserved. In almost all P450s with known structures, the peptide NH of the Cys ligand is the proton donor in an $\mathrm{H}$-bond with a peptide carbonyl $\mathrm{O}$ atom, which forms a tight structure placing the Cys sulfur atom in a position surrounded by

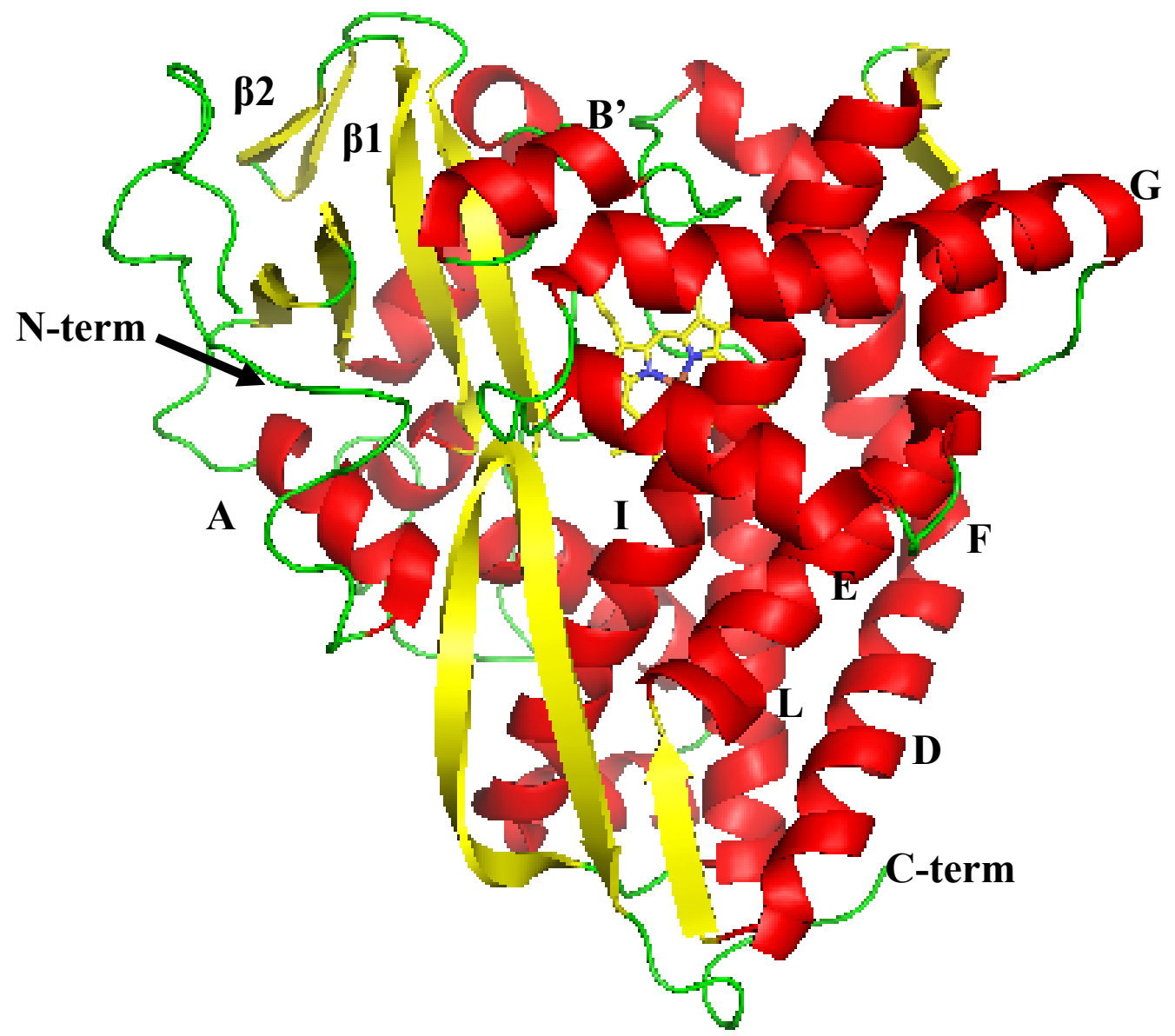

Figure 1.5 Crystal Structure of cytochrome P450cam showing the key elements of secondary structure in different colors. This figure was generated from the crystal data (PDB code 2CPP) using PYMOL (70). 
peptide NH groups forming H-bonds with the thiolate ligand (34). For instance in cytochrome P450cam, the sulfur from Cys357 is surrounded by three amide protons from Leu358, Gly359 and Gln360, which have been reported to stabilize the coordination of the anionic thiolate ligand to the reduced heme iron and also control the "push" effect of the thiolate ligand $(70,72)$. Other P450 enzymes including P450BM3, P450eryF, and P450terp also possess such NH-S hydrogen bonds $(67,73,74)$. This characteristic feature has also been found in other heme thiolate enzymes, such as CPO utilizing two of the three NH-S hydrogen bonds and NOS accepting an additional H-bond from the indole NH of Trp $(20,75)$.

\subsection{HEME PEROXIDASES}

Nonmammalian heme peroxidases and cytochrome P450s are often grouped together because they both contain a single low-potential ferriprotoporphyrin $I X$ (Fig. 1.1) and share similar intermediates in their respective catalytic cycles (76). The first heme peroxidase crystal structure solved was yeast cytochrome $c$ peroxidase $(\mathrm{CcP})$ (77) followed by the first P450 structure (70). Heme peroxidases have played a key role in the development of modern enzymology. They are ubiquitous enzymes that catalyze one electron oxidation of various micro- and macromolecular substrates by utilizing hydrogen peroxide or lipid hydroperoxides as the oxidant, except for chloroperoxidase which is a heme-thiolate protein and performs two-electron oxidations as well $(8,78-80)$. Almost all 
functionally related peroxidases, such as horseradish peroxidase or cytochrome $c$ peroxidase, possess a histidine proximal ligand. The crystal structure studies of the heme peroxidases have firmly established the similarity in their active site architecture: histidine and arginine in the distal side serving as a proton acceptor from hydrogen peroxide and a charge stabilizer, respectively, and heme-bound histidine and hydrogen-bonded aspartic acid in the proximal side of the heme prosthetic group (81-85).

\subsubsection{Catalytic cycles}

Most heme peroxidases follow the reaction scheme as shown in equations (3-5) and share common reaction intermediates, Compound I and Compound II $(8,86,87)$

$$
\begin{aligned}
& \text { Resting state }\left(\mathrm{Fe}^{3+}\right)+\mathrm{H}_{2} \mathrm{O}_{2} \rightarrow \text { Compound } \mathrm{I}\left(\mathrm{Fe}^{4+}=\mathrm{O}, \mathrm{R} \cdot\right)+\mathrm{H}_{2} \mathrm{O} \\
& \text { Compound I }\left(\mathrm{Fe}^{4+}=\mathrm{O}, \mathrm{R} \cdot\right)+\mathrm{SH} \rightarrow \text { Compound } \mathrm{II}\left(\mathrm{Fe}^{4+}=\mathrm{O}\right)+\mathrm{S} \\
& \text { Compound II }\left(\mathrm{Fe}^{4+}=\mathrm{O}\right)+\mathrm{SH} \rightarrow \text { Resting state }\left(\mathrm{Fe}^{3+}\right)+\mathrm{S} \cdot+\mathrm{H}_{2} \mathrm{O}
\end{aligned}
$$

The catalytic cycle begins with the coordination of one equivalent of hydrogen peroxide to the resting ferric peroxidase. The bound peroxide undergoes rapid heterolytic cleavage to produce the active intermediate Cpd I, the ferryl-oxo porphyrin cation radical, where $\mathrm{R}$ is the organic moiety of the heme or an amino acid residue, similar to cytochrome P450s (88). This is a two-electron oxidation/reduction reaction, in which the enzyme is oxidized and hydrogen peroxide is reduced to water. In the following steps, Cpd I is reduced by an organic substrate, usually an aromatic molecule to give a substrate radical, to the resting state in two sequential one-electron transfer reactions via a second 
active intermediate, Cpd II, a ferryl-oxo porphyrin complex $(87,89)$. It is different from P450s which oxidize substrates by inserting one $\mathrm{O}_{2}$-derived oxygen atom into a substrate C-H bond.

The ability to react rapidly with hydrogen peroxide to form Cpd I has distinguished peroxidases from other hemoproteins. This characteristic reactivity has a great deal to do with the influence of the protein environment around the heme center. In most heme peroxidases, there is a highly conserved hisitidine residue in the distal pocket of the active site $(82-85,87,90-93)$. Many studies have shown that the distal His plays a critical role as a general acid-base catalyst in the formation of Cpd I in the peroxidase catalytic cycles $(87,94-97)$. It is proposed that the conserved His facilitates the generation of the initial Fe-OOH complex by deprotonating an approaching hydrogen peroxide as a base and subsequently heterolytically cleaving the $\mathrm{O}-\mathrm{O}$ bond by protonating the departing distal oxygen as an acid (Fig .1.6) (98). Also, a network of hydrogen bonds is reported to assist the distal histidine in its catalytic role in many heme peroxidases such as horseradish peroxidase (Asn-70 H-bonded to His-42), cytochrome $c$ peroxidase, lignin peroxidase and Arthromyces rhamosus peroxidase, and others $(81,84,91,99)$. 


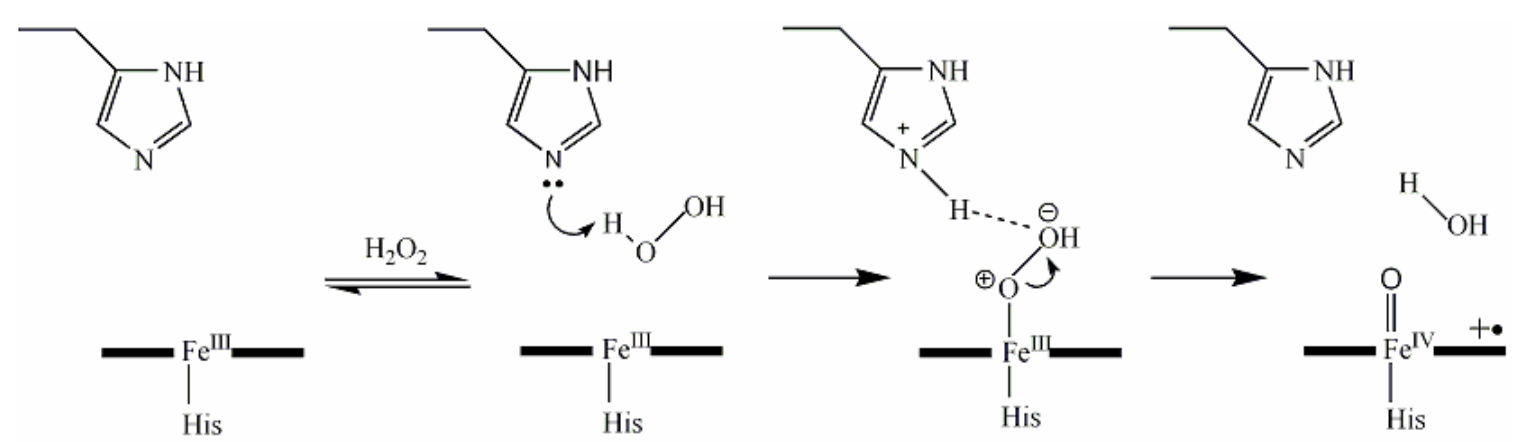

Figure 1.6 Mechanism of Compound I formation in heme peoxidases with distal His.

\subsection{2 "Push-Pull" mechanism compared with P450s}

It is intriguing that heme peroxidases and cytochrome P450 enzymes perform distinct physiological functions while using the same cofactor. Nevertheless, they do share the same key, highly reactive intermediate in their catalytic cycles, Cpd I. It has been reported that the proximal ligand and some distal residues in these heme proteins play significant roles in the promotion of the hereolytic rupture of the O-O bond of dioxygen or peroxides and the following formation of $\mathrm{Cpd} \mathrm{I}$, but through different mechanisms because of different structural features. For many heme peroxidases, a general "push-pull" mechanism has been proposed for this process (Fig. 1.7 (A)) $(80,88$, 100). The "push" effect is provided by the proximal histidine ligand whose electron donor capabilities are enhanced through the H-bonding to a nearby carboxylate group (e.g., Asp). Meanwhile, the distal histidine accepts a proton from the oxygen atom of hydrogen peroxide that binds to the heme iron and transfers it to the other oxygen to form a leaving group. A nearby cationic residue (e.g., protonated Arg) in the distal pocket 
works in concert with the hisitidine to facilitate the O-O bond cleavage. The combination of the distal histidine and arginine has been suggested to provide the "pull" effect (13).

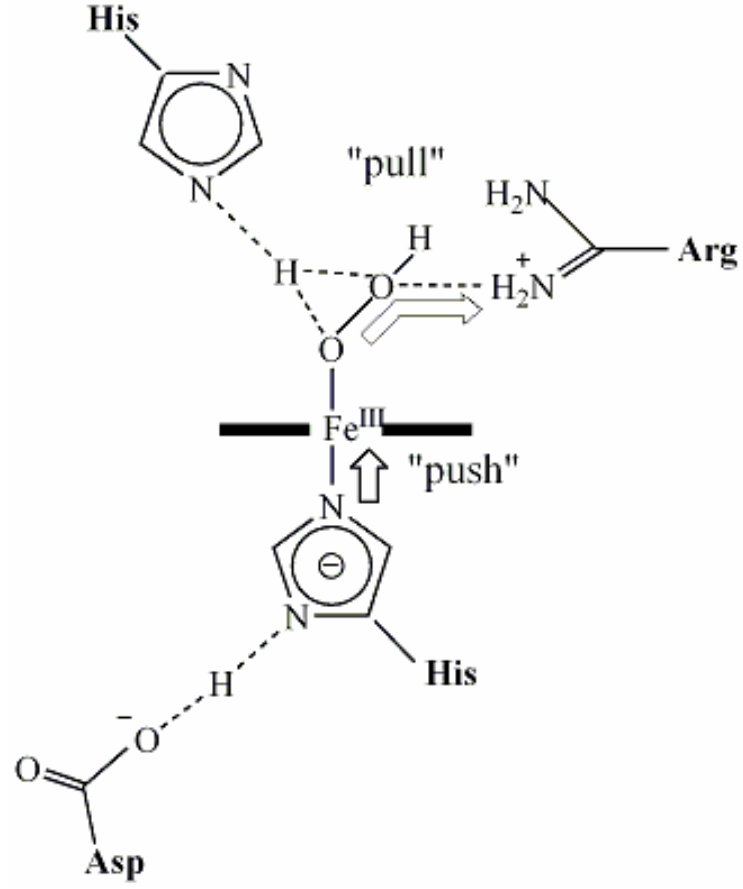

$\mathbf{A}$

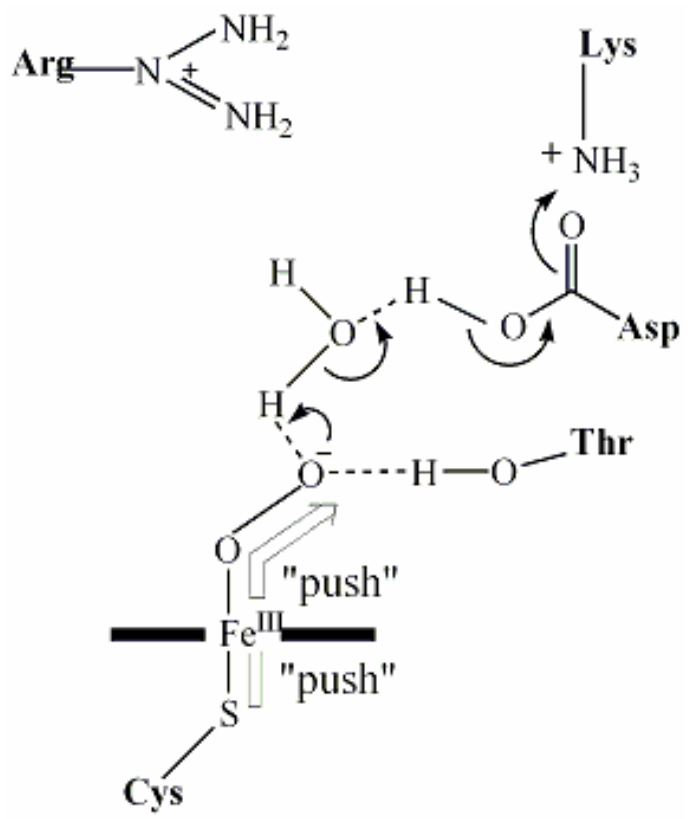

B

Figure 1.7 "Push-pull" mechanisms for O-O bond cleavage of both heme-imidazole protein (A) and heme-thiolate protein (B), such as horseradish peroxidase and P450 respectively. (13)

However, cytochrome P450 lacks such distal machinery. It possesses a cysteine proximal ligand, rather than a histidine typical of most peroxidases. This proximal thiolate ligand has been implicated as a crucial factor to facilitate the heterolytic scission of the O-O bond through its "push" effect by serving as a strong internal electron donor (Fig. 1.7 (B)) $(13,80,101-104)$. The cysteine thiolate enables the protonation of $\mathrm{Cpd} 0$ by the facilitation of Asp and Thr that exist in the protein pocket (14). Once the O-O bond is 
heterolytically cleaved, the electron-releasing axial thiolate ligand would help to stabilize the high-valent nature of Cpd I (13).

\subsubsection{Chloroperoxidase: a P450-peroxidase hybrid}

Chloroperoxidase is an enzyme discovered by Hager and coworkers about 50 years ago from the marine fungus Calduriomyces fumugo (105). CPO serves as one of the most diverse and versatile known heme peroxidases. CPO and cytochromes P450 share a number of physiochemical properties, but they do have clear functional and structural differences (106). In addition to its biological role in halogenation of organic molecules, CPO is also able to use hydrogen peroxide as the oxidant to catalyze one- and two-electron classical peroxidase reactions and P450- and catalase-type reactions under acidic conditions. These enzymatic properties have made CPO a very promising industrial biocatalyst. Cytochrome P450, on the other hand, is a monooxygenase that activates dioxygen to catalyze the hydroxylation of hydrocarbons. Therefore, it is important to define the structural details that differentiate the chemical activities demonstrated by $\mathrm{CPO}$ and $\mathrm{P} 450$.

CPO, with 299 amino acids, is a single polypeptide consisting of eight helical segments and one small $\beta$ pair. Structurally CPO has a P450-like proximal heme environment with cysteinate sulfur as the axial ligand and a peroxidase-like distal heme pocket in that polar residues form the peroxide-binding site, and was thus considered as a P450-peroxidase hybrid (20). Despite the functional properties shared with other 
peroxidases and $\mathrm{P} 450 \mathrm{~s}$, the tertiary structure of $\mathrm{CPO}$ bears little resemblance to either peroxidases or $\mathrm{P} 450 \mathrm{~s}$. In the $\mathrm{CPO}$ distal pocket, though polar as in other peroxidases, a glutamic acid instead of a histidine is responsible for the cleavage of the $\mathrm{O}-\mathrm{O}$ bond in the formation of Cpd I (Fig. 1.8).
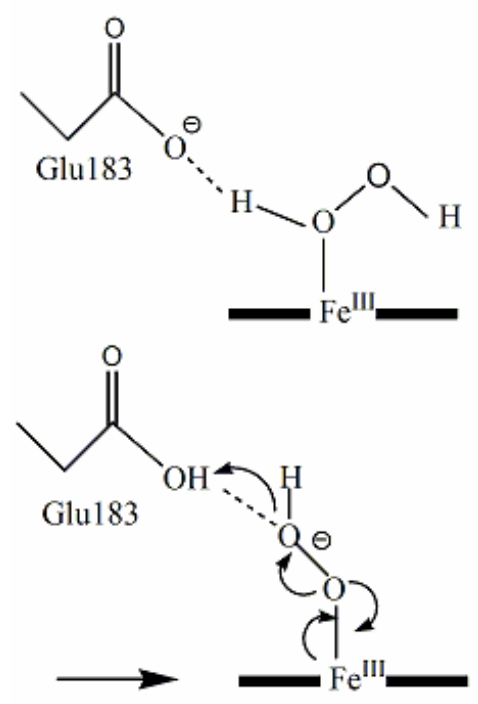
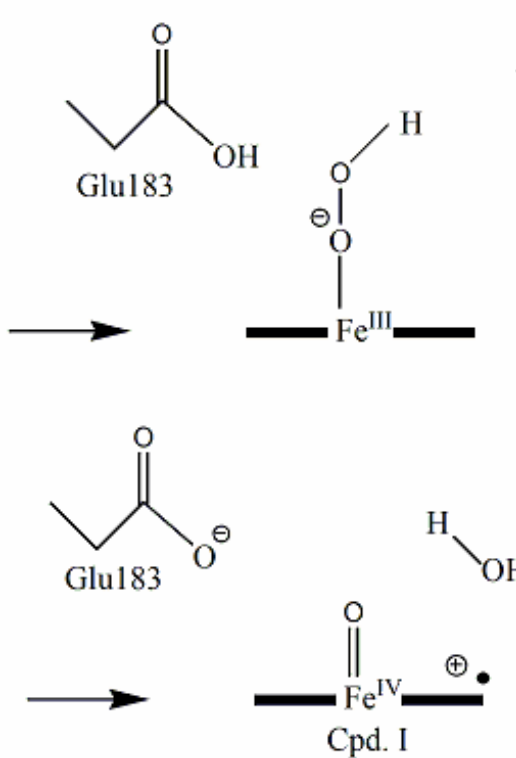

Figure 1.8 Proposed mechanism of compound I formation catalyzed by Chloroperoxidase.

In this mechanism, the activation of hydrogen peroxide is initiated by its binding to the heme in the distal side. The deprotonated Glu183 abstracts a proton from the substrate hydrogen peroxide, and then the formed hydroperoxo-anion binds to the heme iron, yielding a ferric-hydroperoxo species known as Compound 0. Glu183 then protonates the distal oxygen of $\mathrm{Cpd} 0$ and the peroxide $\mathrm{O}-\mathrm{O}$ is cleaved heterolytically to generate $\mathrm{Cpd} \mathrm{I}$ and release a water molecule, similar to other peroxidase mechanisms $(107,108)$. CPO is adept in catalyzing a number of chiral oxidations with high yields and high 
enantioselectivity, in addition to alkyne hydroxylation and heteroatom dealkylation (109-114). The stereoselective ability of CPO in catalysis is suggested to be based on its unique active site structure and unique catalytic mechanism, which make it the promising candidate for industrial catalysts.

\subsection{PROTEIN ENGINEERING OF P450S AND HEME PEROXIDASES}

Heme-containing proteins are primary targets of enzyme engineering because of their distinct activities and versatile functions. The diversity of function and spectroscopic characteristics of this class of proteins have been attributed to the structural components and interactions around the heme prosthetic group, especially the identity of the proximal axial ligation and the residues located in the distal pocket. The hemoproteins have been extensively studied by protein engineering to help test structure-based mechanistic hypotheses and evaluate the roles of important residues in enzyme catalysis, which often involves the change of substrate specificity, reaction selectivity and efficiency of the enzymes.

As mentioned before, cytochromes, globins, and most peroxidases utilize histidine as the proximal ligand, while cytochromes $\mathrm{P} 450$, chloroperoxidase and nictric oxide synthase use a cyseine thiolate ligand. Together with biochemical, biophysical and X-ray crystallographic techniques, site-directed mutagenesis studies have greatly contributed to the understanding of the structure-function relationships of hemoproteins and the key 
structural features governing the variability by achieving structural mimics of different proteins. Substitution of the amino acids in the active site could make it possible to alter the protein axial ligand and to explore the interconversion of functions among hemoproteins, based on structure comparisons. In the following section, the mutagenesis results that demonstrate the importance of protein structure in controlling heme activity and the ability to engineer functions in some important examples of peroxidases and P450 are described.

\subsubsection{Cytochrome $c$ peroxidase H52L \& H175C}

Like many other heme peroxidases, cytochrome $c$ peroxidase $(\mathrm{CcP})$ belongs to the heme-imidazole protein family with a histidine serving as the axial ligand (H175) to the heme iron. In the distal side of the heme group, there is a conserved histidine residue (His52) (81). To investigate the role that histidine plays in the reaction of $\mathrm{CcP}$ with peroxide, His52 was replaced with a nonpolar leucine residue. Although no dramatic changes in the structure or in the accessibility of heme binding sites were observed, the H52L mutant displayed a $10^{5}$-fold decrease in catalytic rate compared with that of the wild type (115). In addition, the reactivity of the H52L mutant with peroxide was $\mathrm{pH}$ dependent and affected by the buffer. The pH-dependent study of this mutant has indicated that the distal histidine plays crucial roles in the rapid reaction of $\mathrm{CcP}$ with peroxide and it is more complex than simply being a base catalyst during the initial formation of Cpd I (116). 
On the other hand, the proximal histidine ligand has been substituted by a cysteine. The successful introduction of a thiolate ligand into a peroxidase system naturally with histidine axial liagtion would shed light on the roles of His and Cys in the catalytic activities of peroxidases by comparing the structure and function of the two heme ligations in similar surroundings. In addition, by monitoring the changes in the structural, functional and spectroscopic properties of the switched thiolate ligation could offer insight into the question about how the thiolate-heme center performs such distinct and diverse functions in various hemoproteins. The initial attempt to prepare a thiolate-ligated $\mathrm{C} c \mathrm{P} \mathrm{H} 175 \mathrm{C}$ mutant led to Cys oxidation to cysteic acid (117). In the crystal structure studies of $\mathrm{P} 450$ and $\mathrm{C} c \mathrm{P}$, it has been shown that $\mathrm{P} 450$ has a hydrophobic environment surrounding the proximal thiolate ligand provided by a conserved Phe residue, while in $\mathrm{C} c \mathrm{P}$ there is a negatively charged Asp which forms hydrogen bonds to the proximal His175 ligand. The Asp235 residue was proposed to be critical in forming a stable heme-thiolate ligation in the resting state of the $\mathrm{C} c \mathrm{P} \mathrm{H} 175 \mathrm{C}$ variant (30).

\subsubsection{P450cam C357H}

In cytochrome P450cam, the crystal structure has demonstrated that the specific cysteine bound to the heme iron is Cys357 (70). To investigate the functional and structural roles of the proximal thiolate ligand in P450cam, the axial Cys357 has been replaced by a histidine $(118,119)$. The importance of the thiolate coordination for the P450 activities has been exemplified by their inactive forms P420, in which the thiolate 
ligation is lost $(120,121)$. The $\mathrm{P} 450$ cam $\mathrm{C} 357 \mathrm{H}$ mutant exhibits greatly decreased camphor oxidation activity, and the heterolytic/ homolytic ratio of the $\mathrm{O}-\mathrm{O}$ bond cleavage in the reaction with cumene hydroperoxide, also the electron transfer rate is much slower than that of the wild type. However, the mutant shows greater peroxidase activity because of the axial ligand alteration. To clarify the roles of the thiolate coordination in the heme-thiolate proteins, the axial histidine of myoglobin has been replaced with a cysteine $(\mathrm{Mb} \mathrm{H} 93 \mathrm{C})$ and the altered thiolate ligand accelerates the heterolytic scission of peroxide O-O bond and enhances the styrene epoxidation activity aided by hydrogen peroxide. These results have confirmed the important roles of the thiolate ligand plays in hemoproteins play in facilitating the O-O bond cleavage to form the active intermediate Cpd I and in protein folding, substrate binding and electron transfer.

\subsubsection{CPO C29H \& CPO E183H}

Chloroperoxidase (CPO) is unique in structure since it shares features with both peroxidases and P450 enzymes. As in cytochromes P450, CPO has a cysteine heme ligand (Cys29) in the proximal side of the heme prosthetic group. However, in contrast to the results of $\mathrm{P} 450 \mathrm{cam} \mathrm{C} 357 \mathrm{H}$, the thiolate coordination in chloroperoxidase is suggested to be not as important as in P450. After substituting the proximal thiolate ligand (Cys 29) with a histidine, the $\mathrm{CPO} \mathrm{C} 29 \mathrm{H}$ was found to retain most (around $80 \%$ ) of its chlorination, peroxidation, epoxidation and catalase activities compared to those of the wild type protein (114). These are surprising observations in consideration of many suggestions for 
the significance of the proximal thiolate ligand in P450. These unexpected results could be interpreted by the different mechanisms of the heterolytic O-O bond cleavage in $\mathrm{CPO}$ and P450 catalytic cycles.

As the results of $\mathrm{CPO} \mathrm{C} 29 \mathrm{H}$ mutant downplayed the importance of thiolate ligation in $\mathrm{CPO}$ activity, it has been suggested that the distal environment of the heme active site plays the major role in controlling the versatile functions of this enzyme. Although, like peroxidases that have polar distal pocket, $\mathrm{CPO}$ does not possess a histidine residue which is normally conserved in the distal helix as the general acid-base catalyst in the formation of Compound I. Instead, a glutamic acid at position 183 distal to the heme group takes over this function, based on the crystal structure of CPO (20). To investigate the role E183 plays in CPO catalysis, it has been replaced by a histidine residue. The results have shown that this substitution is destructive to $\mathrm{CPO}$ activity, especially its chlorination and dismutation activity, which are reduced by $85 \%$ and $50 \%$, respectively (122). This indicated that Glu-183 is important but not essential to the chlorination and the catalase activity of CPO. However the epoxidation activity of the CPO E183H mutant was surprisingly increased by about 2.5 times than that of the wild type. The crystal structure of CPO identified a proton shuttle among Glu183, His105, and Asp106. Replacement of glutamic acid with a hisitidine could partially destroy the triad, resulting in the inhibition of chlorination activity but acceleration epoxidation activity. 
In summary, mutagenesis studies have shown that the axial ligands in P450s and peroxidases do play essential roles in their catalysis. Key factors that determine the functional specificity of hemoproteins include the nature of the proximal ligands as well as the architecture of the distal site. The remainder of the dissertation details my effort to tailor heme-thiolate proteins, specifically cytochrome P450cam, into efficient biocatalysts with high specificity and selectivity by redesigning the protein through change of residues in the distal pocket, in an effort to elucidate the key structural and functional roles of critical amino acids in this protein.

\subsection{REFERENCES}

1. Thomson, A. J., Gray, H. B. (1998) Bioinorganic chemistry: editorial overview, Curr. Opin. Chem. Biol. 2, 155-158.

2. Lu, Y., Yeung, N., Sieracki, N., and Marshall, N. M. (2009) Design of functional metalloproteins, Nature 460, 855-862.

3. Williams, R. J. P. (1987) Missing information in bio-inorganic chemistry, Coord. Chem. Rev. 79, 175-193.

4. Turano, P., and Lu, Y. (2001) in Handbook on Metalloproteins (Bertini, I., Sigel, H. \& Sigel, A., Ed.), pp 269-356, Dekker, New York.

5. Bertini, I., Gray, H. B., Lippard, S. J. \& Valentine, J. S. (1994) Bioinorganic Chemistry Univ. Sci. Books, Mill Valley, CA.

6. Lippard, S. J., and Berg, J.M. (1994) Principles of Bioinorganic Chemistry, Univ. Sci. Books, Mill Valley, CA. 
7. Lu, Y., Berry, S. M., and Pfister, T. D. (2001) Engineering novel metalloproteins: Design of metal-binding sites into native protein scaffolds, Chemical Reviews 101, 3047-3080.

8. Dunford, H. B. (1999) Heme Peroxidases, John Wiley \& Sons, New York.

9. Raphael, A. L. a. G., H. B. (1991) Semisynthesis of Axial-Ligand (Position 80) Mutants of Cytochrome c, J. Am. Chem. Soc. 113, 1038-1040.

10. Antonini, E. a. B., M. (1971) Hemoglobin and Myoglobin in Their Reactions with Ligands, Elsevier, New York.

11. Perutz, M. F. (1989) Myoglobin and haemoglobin: role of distal residues in reactions with haem ligands, Trends. Biochem. Sci. 14, 42-44.

12. Ortiz de Montellano, P. R. (1998) Heme oxygenase mechanism: Evidence for an electrophilic, ferric peroxide species, Acc. Chem. Res. 31, 543-549.

13. Sono, M. R., M. P.; Coulter, E. D.; and Dawson, J. H. (1996) Heme-Containing Oxygenases, Chemical Reviews 96, 2841-2887.

14. Ortiz de Montellano, P. R. (2005) Cytochrome P450: structure, mechanism, and biochemistry, 3 ed., Kluwer Academic/Plenum Publishers, New York.

15. Poulos, T. L., Li, H. Y., Raman, C. S., and Schuller, D. J. (2001) Structures of gas-generating heme enzymes: Nitric oxide synthase and heme oxygenase, in Advances in Inorganic Chemistry, Vol 51, pp 243-293.

16. Chan, M. K. (2000) Heme protein biosensors, J. Porphyrins Phthalocyanines 4, 358-361.

17. Omura, T. (2005) Heme-thiolate proteins, Biochem. Biophys. Res. Commun. 338, 404-409.

18. Udit, A. K., and Gray, H. B. (2005) Electrochemistry of heme-thiolate proteins, Biochemical and Biophysical Research Communications 338, 470-476. 
19. Sono, M., Dawson. J. H., Hall, K., Hager, L. P. (1986) Ligand and Halide Binding Properties of Chloroperoxidase: Peroxidase-Type Active Site Heme Environment with Cytochrome P-450 Type Endogenous Axial Ligand and Spectroscopic Properties, Biochemistry 25, 347-356.

20. Sundaramoorthy, M., Terner, J., and Poulos, T. L. (1995) The crystal structure of chloroperoxidase: A heme peroxidase-cytochrome P450 functional hybrid, Structure 3, 1367-1377.

21. Hollengerg, P. F., Hager, L. P. (1973) The P450-nature of the carbon monoxide complex of ferrous chloroperoxidase, J. Biol. Chem. 248, 2630-2633.

22. White, K. A., Marletta, M. A. (1992) Nitric oxide synthase is a cytochrome P-450 type hemoprotein, Biochemistry 31, 6627-6631.

23. Sono, M., Stuehr, D. J., Ikeda-Saito, M., Dawson, J. H. (1995) Identification of Nitric Oxide Synthase as a Thiolate-ligated Heme Protein Using Magnetic Circular Dichroism Spectroscopy: COMPARISON WITH CYTOCHROME P-450-CAM AND CHLOROPEROXIDASE, J. Biol. Chem. 270, 19943-19948.

24. Nakajima, H., Nakagawa, E., Kobayashi, K., Tagawa, S., and Aono, S. (2001) Ligand-switching intermediates for the CO-sensing transcriptional activator CooA measured by pulse radiolysis, Journal of Biological Chemistry 276, 37895-37899.

25. Reynolds, M. F., Shelver, D., Kerby, R. L., Parks, R. B., Roberts, G. P., Burstyn, J. N. (1998) EPR and Electronic Absorption Spectroscopies of the CO-Sensing CooA Protein Reveal a Cysteine-Ligated Low-Spin Ferric Heme J. Am. Chem. Soc. 120, 9080-9081.

26. Meier, M., Janosik, M., Kery, V., Kraus, J. P., and Burkhard, P. (2001) Structure of human cystathionine $\beta$-synthase: a unique pyridoxal 5'-phosphate-dependent heme protein., EMBO Journal 20, 3910-3916.

27. Ojha, S., Hwang, J., Kabil, O., Penner-Hahn, J. E., and Banerjee, R. (2000) Characterization of the Heme in Human Cystathionine $\beta$-Synthase by X-ray Absorption and Electron Paramagnetic Resonance Spectroscopies, Biochemistry $39,10542-10547$.

28. Matsui, T., Nagano, S., Ishimori, K., Watanabe, Y., and Morishima, I. (1996) Preparation and reactions of myoglobin mutants bearing both proximal cysteine 
ligand and hydrophobic distal cavity: Protein models for the active site of P-450, Biochemistry 35, 13118-13124.

29. Adachi, S., Nagano, S., Ishimori, K., Watanabe, Y., Morishima, I., Egawa, T., Kitagawa, T., and Makino, R. (1993) Roels of Proximal Ligand in Heme-Proteins - Replacement of Proximal Histidine of Human Myoglobin with Cysteine and Tyrosine by Site-Directed Mutagenesis as Models for P-450, Chloroperoxidase, and Catalase, Biochemistry 32, 241-252.

30. Sigman, J. A., Pond, A. E., Dawson, J. H., and Lu, Y. (1999) Engineering cytochrome c peroxidase into cytochrome P450: A proximal effect on heme-thiolate ligation, Biochemistry 38, 11122-11129.

31. Liu, Y., Moenne-Loccoz, P., Hildebrand, D. P., Wilks, A., Loehr, T. M., Mauk, A. G., and Ortiz de Montellano, P. R. (1999) Replacement of the Proximal Histidine Iron Ligand by a Cysteine or Tyrosine Converts Heme Oxygenase to an Oxidase, Biochemistry 38, 3733-3743.

32. Vetter, S. W., Terentis, A. C., Osborne, R. L., Dawson, J. H., and Goodin, D. B. (2009) Replacement of the axial histidine heme ligand with cysteine in nitrophorin 1: spectroscopic and crystallographic characterization, Journal of Biological Inorganic Chemistry 14, 179-191.

33. Wang, W., Lu, J., Yao, P., Xie, Y., and Huang, Z. (2003) The distinct heme coordination environments and heme-binding stabilities of His39Ser and His39Cys mutants of cytochrome b5, Protein Engineering 16, 1047-1054.

34. Sigel, A., Sigel, H., and Sigel, R. K.O. (2007) The ubiquitous roles of cytochrome P450 proteins in Metal Ions in Life Sciences (Sigel, A., Sigel, H., and Sigel, R. K.O. , Ed.), Wiley, Chichester.

35. Lewis, D. F. V. (1996) Cytochromes P450: Structure, Function, and Mechanism, Taylor \& Francis, London.

36. Lechner, M. C., Ed. (1993) Cytochrome P-450: Biochemistry, Biophysics, and Molecular Biology, John Libbey Eurotext, Paris.

37. Waterman, M. R., Johnson, E. F., Eds. (1991) Cytochrome P450 (Part A), in Methods in Enzymology, Academic Press, San Diego. 
38. Johnson, E. F., Waterman, M. R., Eds. (1996) Cytochrome P450 (Part B), in Methods in Enzymology, Academic Press, San Diego.

39. Phillips, I. R., Shephard, E. A., Eds. (1998) Cytochrome P450 Protocols, in Methods in Molecular Biology, Humana Press, Totowa

40. Denisov, I. G., Makris, T. M., Sligar, S. G., and Schlichting, I. (2005) Structure and chemistry of cytochrome P450, Chemical Reviews 105, 2253-2277.

41. Hasemann, C. A., Kurumbail, R.G., Boddupalli, S.S., Peterson, J.A. and Deisenhofer, J. (1995) Structure and function of cytochromes P450: a comparative analysis of three crystal structures, Structure 3, 41-62.

42. Wong, L. L. (1998) Cytochrome P450 monooxygenases, Curr. Opin. Chem. Biol. $2,263-268$.

43. Guengerich, F. P. (1991) Reactions and Significance of Cytochrome P-450 Enzymes, Journal of Biological Chemistry 266, 10019-10022.

44. Sligar, S. G., Makris, T. M., Denisov, I. G. (2005) Thirty years of microbial P450 monooxygenase research: Peroxo-heme intermediates - The central bus station in heme oxygenase catalysis, Biochemical and Biophysical Research Communications 338, 346-354.

45. Nelson, D. R. (2009) The Cytochrome P450 Homepage, Human Genomics 4, $59-65$.

46. Mansuy, D. (1998) The great diversity of reactions catalyzed by cytochromes P450, Comp Biochem Physiol C Pharmacol Toxicol Endocrinol 121, 5-14.

47. Sligar, S. G., Gunsalus I.C. (1976) A thermodynamic model of regulation: modulation of redox equilibria in camphor monoxygenase, Proc Natl Acad Sci USA 73, 1078-1082.

48. Daff, S. N., Chapman, S. K., Turner, K. L., Holt, R. A., Govindaraj, S., Poulos, T. L., and Munro, A. W. (1997) Redox Control of the Catalytic Cycle of Flavocytochrome P-450 BM3, Biochemistry 36, 13816-13823. 
49. Newcomb, M., Zhang, R., Chandrasena, R. E. P., Halgrimson, J. A., Horner, J. H., Makris, T. M., and Sligar, S. G. (2006) Cytochrome P450 Compound I, Journal of the American Chemical Society 128, 4580-4581.

50. Imai, M., Shimada, H., Watanabe, Y., Matsuthima-Hibiya, Y., Makino, R., Koga, H., Horiuchi, T., Ishimura,Y. (1989) Uncoupling of the cytochrome P-450cam monooxygenase reaction by a single mutation, threonine-252 to alanine or valine: A possible role of the hydroxy amino acid in oxygen activation, Proc Natl Acad Sci USA 86, 7823-7827.

51. Harris, D. L., Loew, G.H. (1994) A Role for Thr 252 in Cytochrome P450cam Oxygen Activation, J. Am. Chem. Soc. 116, 11671-11674.

52. Schlichting, I., Berendzen, J., Chu, K., Stock, A. M., Maves, S. A., Benson, D. E., Sweet, B. M., Ringe, D., Petsko, G. A., and Sligar, S. G. (2000) The catalytic pathway of cytochrome P450cam at atomic resolution, Science 287, 1615-1622.

53. Capdevila, J., Estabrook, R.W., Prough, R.A. (1980) Differences in the mechanism of NADPH and cumene hydroperoxide-supported reactions of cytochrome P-450, Arch. Biochem. Biophys. 200, 186-195.

54. McCarthy, M. B., White, R.E. (1983) Functional differences between peroxidase compound I and the cytochrome $\mathrm{P}-450$ reactive oxygen intermediate, J. Biol. Chem. 258.

55. Coon, M. J., Blake, R.C. 2nd, White, R.E., Nordblom, G.D. (1990) Assays for cytochrome P-450 peroxygenase activity, Methods Enzymol 186, 273-278.

56. Cirino, P. C., and Arnold, F. H. (2002) Regioselectivity and activity of cytochrome P450 BM-3 and mutant F87A in reactions driven by hydrogen peroxide, Advanced Synthesis \& Catalysis 344, 932-937.

57. Joo, H., Lin, Z. L., and Arnold, F. H. (1999) Laboratory evolution of peroxide-mediated cytochrome P450 hydroxylation, Nature 399, 670-673.

58. Cirino, P. C., and Arnold, F. H. (2003) A self-sufficient peroxide-driven hydroxylation biocatalyst, Angew. Chem. Int. Ed. 42, 3299-3301. 
59. Prasad, S., and Mitra, S. (2004) Substrate modulates compound I formation in peroxide shunt pathway of Pseudomonas putida cytochrome P450(cam), Biochemical And Biophysical Research Communications 314, 610-614.

60. Matsui, K., Shibutani, M., Hase, T., Kajiwara, T. (1996) Bell pepper fruit fatty acid hydroperoxide lyase is a cytochrome P450 (CYP74B), FEBS Lett 394, 21-24.

61. Song, W. C., Funk, C.D., Brash, A.R. (1993) Molecular cloning of an allene oxide synthase: a cytochrome P450 specialized for the metabolism of fatty acid hydroperoxides, Proc Natl Acad Sci USA 90, 8519-8523.

62. Miyata, A., Hara, S., Yokoyama, C., Inoue, H., Ullrich, V., Tanabe, T. (1994) Molecular cloning and expression of human prostacyclin synthase, Biochem. Biophys. Res. Commun. 200, 1728-1734.

63. Matsunaga, I., and Shir, Y. (2004) Peroxide-utilizing biocatalysts: structural and functional diversity of heme-containing enzymes, Current Opinion in Chemical Biology 8, 127-132.

64. Shoji, O., Fujishiro, T., Nakajima, H., Kim, M., Nagano, S., Shiro, Y., Watanabe, Y. (2007) Hydrogen Peroxide-Dependent Monooxygenations by Tricking the Substrate Recognition of Cytochrome P450BS $\beta$, Angew. Chem. Int. Ed. 46, 3656-3659.

65. Lee, D., Yamada, A., Sugimoto, H., Matsunaga, I., Ogura, H., Ichihara, K., Adachi, S., Park, S. and Shiro, Y. (2003) Substrate Recognition and Molecular Mechanism of Fatty Acid Hydroxylation by Cytochrome P450 from Bacillus subtilis, J. Biol. Chem. 278, 9761-9767.

66. Matsunaga, I., Sumimoto, T., Ayata, M., Ogura, H. (2002) Functional modulation of a peroxygenase cytochrome P450: novel insight into the mechanisms of peroxygenase and peroxidase enzymes, FEBS Lett 528, 90-94.

67. Hasemann, C. A., Kurumbail, R.G., Boddupalli, S.S., Peterson, J.A., and Deisenhofer, J. (1995) Structure and function of cytochromes P450:a comparative analysis of three crystal structures, Structure 3, 41-62.

68. Graham, S. E., Peterson, J.A. (1999) How Similar Are P450s and What Can Their Differences Teach Us?, Arch. Biochem. Biophys. 369, 24-29. 
69. Johnson, E. F., and Stout, C.D. (2005) Structural diversity of human xenobiotic-metabolizing cytochrome P450 monooxygenases, Biochem. Biophys. Res. Commun. 338, 331-336.

70. Poulos, T. L., Finzel, B. C., and Howard, A. J. (1987) HIGH-RESOLUTION CRYSTAL-STRUCTURE OF CYTOCHROME-P450CAM, Journal of Molecular Biology 195, 687-700.

71. Poulos, T. L., Finzel, B. C., Gunsalus, I. C., Wagner, G. C., and Kraut, J. (1985) THE 2.6-A CRYSTAL-STRUCTURE OF PSEUDOMONAS-PUTIDA CYTOCHROME-P-450, Journal of Biological Chemistry 260, 6122-6130.

72. Poulos, T. L. (1996) The role of the proximal ligand in heme enzymes, J. Biol. Inorg. Chem. 1, 356-359.

73. Ravichandran, K. G., Boddupalli, S. S., Hasemann, C. A., Peterson, J. A., Deisenhofer, J. (1993) Crystal structure of hemoprotein domain of P450BM-3, a prototype for microsomal P450's, Science 261, 731-736.

74. Cupp-Vickery, J., Poulos, T. L. . (1995) Structure of cytochrome P450yp involved in erythromycin biosynthesis, Nat. Struct. Biol. 2, 144-153.

75. Crane, B. R., Arvai, A. S., Gachhui, R., Wu, C., Ghosh, D. K., Getzoff, E.D., Stuehr, D. J., Tainer, J. A. . (1997) The Structure of Nitric Oxide Synthase Oxygenase Domain and Inhibitor Complexes, Science 278, 425-431.

76. Poulos, T. L. (2000) Peroxidase and cytochrome P450 structures, in Porphyrin Handbook (Kadish, K. M., Smith, K. M., Guilard, R., Ed.), pp 189-218, Academic Press, San Diego, Calif.

77. Poulos, T. L., Freer, S. T., Alden, R. A., Edwards, S. L., Skogland, U., Takio, K., Eriksson, B., Xuong, N. H., Yonetani, T., and Kraut, J. (1980) Crystal-Structure of Cytochrome-c Peroxidase, J. Biol. Chem. 255, 575-580.

78. Welinder, K. G. (1992) Superfamily of plant, fungal and bacterial peroxidases, Curr. Opin. Struct. Biol. 2, 388-393.

79. Poulos, T. L. (1987) Heme enzyme crystal structures, Adv. Inorg. Biochem. 7, $1-36$. 
80. Dawson, J. H. (1988) Probing structure-function relations in hemecontaining oxygenases and preoxidases, Science 240, 433-439.

81. Finzel, B. C., Poulos, T. L., and Kraut, J. (1984) Crystal structure of yeast cytochrome c peroxidase refined at 1.7-A resolution, J. Biol. Chem. 259, 13027-13036.

82. Patterson, W. R., and Poulos, T. L. (1995) Crystal structure of recombinant pea cytosolic ascorbate peroxidase, Biochemistry 34, 4331-4341.

83. Gajhede, M., Schuller, D. J., Henriksen, A., Smith, A. T., and Poulos, T. L. (1997) Crystal Structure of Horseradish Peroxidase C at $2.15 \AA$ Resolution, Nat. Struct. Biol. 4, 1032-1038.

84. Poulos, T. L., Edwards, S. L., Wariishi, H., and Gold, M. G. (1993) Crystallographic refinement of lignin peroxidase at 2 A, J. Biol. Chem. 268, 4429-4440.

85. Sundaramoorthy, M., Kishi, K., Gold, M. H., and Poulos, T. L. (1994) The crystal structure of manganese peroxidase from Phanerochaete chrysosporium at 2.06-A resolution, J. Biol. Chem. 269, 32759-32767.

86. Li, H. Y., Poulos, T.L. (1994) Structural variation in heme enzymes: a comparative analysis of peroxidase and P450 crystal structures, Structure 2, 461-464.

87. Tanaka, M., Ishimori, K., Mukai, M., Kitagawa, T., and Morishima, I. (1997) Catalytic activities and structural properties of horseradish peroxidase distal His42-> Glu or Gln mutant, Biochemistry 36, 9889-9898.

88. Al-Mustafa, J., Kincaid, J.R. (1994) Resonance Raman Study of Cyanide-Ligated Horseradish Peroxidase. Detection of Two Binding Geometries and Direct Evidence for the "Push-Pull" Effect, Biochemistry 33, 2191-2197.

89. Yamada, H., and Yamazaki, I. . (1974) Proton balance in conversions between five oxidation-reduction states of horseradish peroxidase, Arch. Biochem. Biophys. $165,728-738$. 
90. Fukuyanma, K., Kunishima, N. Amada, F., Kubota, T., Matsubarai, H. (1995) Crystal Structures of Cyanide- and Triiodide-bound Forms of Arthromyces ramosus Peroxidase at Different pH Values, J. Biol. Chem. 270, 21884-21892.

91. Kunishima, N., Fukuyama, K., Matsubara, H., Hatanaka, H., Shibano, Y., and Amachi, T. (1994) Crystal structure of the fungal peroxidase from Arthromyces ramosus at $1.9 \AA$ resolution: Structural comparisons with the lignin and cytochrome c peroxidases, J. Mol. Biol. 235, 331-344.

92. Edwards, S. L., Raag, R., Wariishi, H., Gold, M. H., and Poulos, T. L. (1993) Crystal structure of lignin peroxidase, Proc. Natl. Acad. Sci. U.S.A. 90, 750-754.

93. Schuller, D. J., Ban, N., van Huystee, R. B., McPherson, A., and Poulos, T. L. (1996) The crystal structure of peanut peroxidase, Structure 4, 311-321.

94. Newmyer, S. L. a. O. d. M., P.R. (1996) Rescue of the Catalytic Activity of an H42A Mutant of Horseradish Peroxidase by Exogenous Imidazoles, J. Biol. Chem. 271, 14891-14896.

95. Howes, B. D., RodriguezLopez, J. N., Smith, A. T., and Smulevich, G. (1997) Mutation of distal residues of horseradish peroxidase: Influence on substrate binding and cavity properties, Biochemistry 36, 1532-1543.

96. Foshay, M. C., Vitello, L.B., and Erman, J.E. (2009) Relocation of the Distal Histidine in Cytochrome c Peroxidase: Properties of $\mathrm{CcP}(\mathrm{W} 51 \mathrm{H})$, $\mathrm{CcP}(\mathrm{W} 51 \mathrm{H} / \mathrm{H} 52 \mathrm{~W})$, and $\mathrm{CcP}(\mathrm{W} 51 \mathrm{H} / \mathrm{H} 52 \mathrm{~L})$, Biochemistry 48, 5417-5425.

97. Poulos, T. L., and Kraut, J. (1980) THE STEREOCHEMISTRY OF PEROXIDASE CATALYSIS, J. Biol. Chem. 255, 8199-8205.

98. Poulos, T. L., and Kraut, J. (1980) The Sterochemistry of Peroxidase Catalysis, J. Biol. Chem. 255, 8199-8205.

99. Newmyer, S. L., and Ortiz de Montellano, P. R. (1996) Rescue of the Catalytic Activity of an H42A Mutant of Horseradish Peroxidase by Exogenous Imidazoles, J. Biol. Chem. 271, 14891-14896.

100. Poulos, T. L. (1988) Heme enzyme crystal structures, Adv. Inorg. Biochem. 7, $1-36$. 
101. Kimata, Y., Shimada, H., Hirose, T., Ishimura, Y. (1995) Role of Thr-252 in cytochrome P450cam: a study with unnatural amino acid mutagenesis, Biochem. Biophys. Res. Commun. 209, 96-102.

102. Gerber, N., C., Sligar, S. G. (1994) A role for Asp-251 in cytochrome P-450cam oxygen activation, J. Biol. Chem. 269, 4260-4266.

103. Gerber, N. C., Sligar, S. G. (1992) Catalytic mechanism of cytochrome P-450: evidence for a distal charge relay, J. Am. Chem. Soc. 114.

104. Dawson, J. H., Holm, R. H., Trudell, J. R., Barth, G., Linder, R. E., Bunnenberg, E., Djerassi, C., Tang, S. C. (1976) Oxidized cytochrome P-450. Magnetic circular dichroism evidence for thiolate ligation in the substrate-bound form. Implications for the catalytic mechanism, J. Am. Chem. Soc. 98, 3707-3709.

105. Morris, D. R., Hager, L. P. (1966) Chloroperoxidase: I. Isolation and Properties of the Crystalline Glycoprotein J. Biol. Chem. 241, 1763-1768.

106. Dawson, J. H., and Sono, M. (1987) Cytochrome P-450 and Chloroperoxidase: Thiolate-Ligated Heme Enzymes. Spectroscopic Determination of Their Active Site Structures and Mechanistic Implications of Thiolate Ligation, Chemical Reviews 87, 1255-1276.

107. Wang, X., Tachikawa, H., Yi, X., Manoj, K. M., and Hager, L. P. (2003) Two-dimensional NMR Study of the Heme Active Site Structure of Chloroperoxidase, J. Biol. Chem. 278, 7765-7774.

108. Kuhnel, K., Blankenfeldt, W., Terner, J., Schlichting, I. (2006) Crystal Structures of Chloroperoxidase with Its Bound Substrates and Complexed with Formate, Acetate, and Nitrate, J. Biol. Chem. 281, 23990-23998.

109. Hu, S. H., Hager, L.P. (1999) Highly enantioselective propargylic hydroxylations catalyzed by chloroperoxidase, J. Am. Chem. Soc. 121, 872-873.

110. Hu, S. H., Hager, L.P. (1999) Asymmetric epoxidation of functionalized cis-olefins catalyzed by chloroperoxidase, Tetrahedron Lett. 40, 1641-1644.

111. Kedderis, G. L., Hollenberg, P.F. (1984) Peroxidase-catalyzed N-demethylation reactions. Substrate deuterium isotope effects, J. Biol. Chem. 259, 3663-3668. 
112. Kedderis, G. L., Koop, D.R., Hollenberg, P.F. (1980) N-Demethylation reactions catalyzed by chloroperoxidase, J. Biol. Chem. 255, 10174-10182.

113. Hager, L. P., Lakner F.J., Basavapathruni, A. (1998) Chiral synthons via chloroperoxidase catalysis, J. Mol. Catal. B: Enzym. 5, 95-101.

114. Yi, X., Mroczko, M., Manoj, K.M., Wang, X., Hager, L.P. (1999) Replacement of the proximal heme thiolate ligand in chloroperoxidase with a histidine residue, Proc Natl Acad Sci USA 96, 12412-12417.

115. Erman, J. E., Vitello, L. B., Miller, M. A., Shaw, A., Brown, K. A., and Kraut, J. (1993) Histidine-52 is A Critical Residue for Rapid Formation of Cytochrome-c Peroxidase Compound-I, Biochemistry 32, 9798-9806.

116. Palamakumbura, A. H., Vitello, L. B., and Erman, J. E. (1999) Oxidation of the His-52 ->Leu Mutant of Cytochrome c Peroxidase by p-Nitroperoxybenzoic Acid: Role of the Distal Histidine in Hydroperoxide Activation, Biochemistry 38, $15653-15658$.

117. Choudhury, K., Sundaramoorthy, M., Hickman, A., Yonetani, T., Woehl, E., Dunn, M. F., and Poulos, T. L. (1994) Role of the Proximal Ligand in Peroxidase Catalysis J. Biol. Chem. 269, 20239-20249.

118. Auclair, K., Moenne-Loccoz, P., and Ortiz de Montellano, P. R. (2001) Roles of the Proximal Heme Thiolate Ligand in Cytochrome P450cam, J. Am. Chem. Soc. $123,4877-4885$.

119. Yoshioka, S., Takahashi, S., Hori, H., Ishimori, K., and Morishima, I. (2001) Proximal cysteine residue is essential for the enzymatic activities of cytochrome P450cam, Eur. J. Biochem. 268, 252-259.

120. Yu, C.-A., Gunsalus, I .C. (1974) Cytochrome P-450cam: II. Interconversion with P-420, J. Biol. Chem. 249, 102-106.

121. Martinis, S. A., Blanke, S. R., Hager, L. P., Sligar, S. G., Hoa, G. H. B., Rux, J. J., and Dawson, J. H. (1996) Probing the heme iron coordination structure of pressure-induced cytochrome P420cam, Biochemistry 35, 14530-14536. 
122. Yi, X., Conesa, A., Punt, J. P., Hager, L. P. (2003) Examining the Role of Glutamic Acid 183 in Chloroperoxidase Catalysis, J. Biol. Chem. 278, 13855-13859. 


\section{Chapter II. SINGLE AMINO ACID SWITCH TURNS P450CAM FROM A MONOOXYGENASE INTO AN EFFICIENT PEROXIDASE}

\subsection{SUMMARY}

Most heme peroxidases contain a highly conserved histidine that serves as the general acid-base catalyst at the distal side of the heme prosthetic group in the formation of compound I. In contract, cytochromes P450 do not have such obvious acid-base groups in their hydrophobic distal heme pockets. To explore the possibility of engineering cytochrome P450cam into an efficient peroxygenase via the "peroxide-shunt" pathway, Val247 located closely to the heme iron in the distal helix has been substituted with a histidine residue. The V247H mutant shows essentially identical optical spectral features compared to those of wild type P450cam in the absence of substrate camphor, suggesting that the heme-Cys ligation in the parent protein is kept intact in the mutant. Surprisingly this mutant does not bind camphor like the wild type, possibly due to unfavorable conformational changes in the distal helix which prevents camphor from entering the substrate binding site in the same way. However, the V247H mutant has acted as a self-sufficient enzyme with significantly higher peroxidase activity compared with wild type protein, showing even higher activity than chloroperoxidase. Various spectroscopic studies including UV, RR, CD and NMR coupled with electrochemical studies have 
provided insights into the structural changes that correlate with the observed catalytic activities of this mutant.

\subsection{INTRODUCTION}

Cytochromes P450 constitute a superfamily of heme-containing monooxygenases that are found ubiquitously in many bacteria, all archaea, fungi and many higher eukaryotes. The P450 enzymes are involved in the activation of dioxygen for the insertion or addition of one atom of oxygen to a wide variety of substrates, making them crucial to many biotransformations such as steroid hormone biosynthesis, drug metabolism, detoxification of xenobiotics, and molecular signaling (1-3). The reactions catalyzed by P450s include epoxidation, dealkylation, hydroxylation, dehalogenation, and heteroatom oxidation (4). The most distinctive structural feature of cytochromes P450 is the coordination of a deprotonated cysteine to the heme iron as the proximal ligand, while many other hemoproteins possess a histidine at the proximal side. Thus P450s belong to the family of so-called heme-thiolate proteins, together with choloroperoxidase (CPO) from Caldariomyces fumago, nitric oxide synthase (NOS), cystathionine $\beta$-synthase, and etc. The proximal thiolate ligand in P450s has been proposed to play a vital mechanistic role in providing the "push" effect which enables the heterolytic O-O bond cleavage to generate activated iron (IV)-oxo porphyrin radical cation species commonly called CpdI, leading to subsequent substrate oxidation (5-7). 
The typical P450 catalytic cycle involves substrate binding, dioxygen binding, two-electron transfers, followed by CpdI formation and substrate oxidation. With a few exceptions, the catalysis of the diverse reactions by $\mathrm{P} 450$ s all require an external source of reducing equivalents and auxiliary proteins (e.g., putidaredoxin, and puridaredoxin reductase for P450cam). The requirement of expensive cofactors and the complication caused by additional proteins is the limitation to the application of P450s as oxygenases. Some P450s are capable of accepting hydrogen peroxide or an organic peroxy compound as an oxidant to catalyze oxygen insertion through the so-called "peroxide-shunt" pathway in the absence of electron transport proteins and the NAD(P)H cofactor $(8-13)$. However, P450s are typically not efficient in their utilization of hydrogen peroxide, the natural substrate for peroxidases, as the source of oxygen atom in the monooxygenation reactions.

Serving as the prototype member in the superfamily, cytochrome P450cam from Pseudomonus putida has been extensively studied using various physical and chemical techniques. It catalyzes the regio- and stereo-specific hydroxylation of its natural substrate $d$-camphor to 5-exo-hydroxy-camphor (15). A great deal of effort has been made to suitably engineer this enzyme by directed evolution and active site modification for the oxygenation of significant unnatural substrates. It is still quite important to understand the roles of the active-site components in the reactivity, stability, specificity and substrate recognition of this enzyme after its discovery decades ago. 
To explore the possibility of converting P450cam into an efficient self-sufficient peroxidase, we attempted to modify the heme distal side elements that play crucial mechanistic roles in this protein's catalytic functions. In most heme peroxidases, a distal histidine is highly conserved in the active site and serves as a general acid-base catalyst that is necessary for the formation of $\mathrm{CpdI}$ by donating a proton to the $\mathrm{Fe}^{3+}-\mathrm{OOH}$ moiety to promote the heterolytic cleavage of the $\mathrm{O}-\mathrm{O}$ bond in the peroxidase reaction cycles (16-19). It has also been proposed that a functional nitrogen (such as the nitrogen of His42 in HRP or His52 in $\mathrm{C} c \mathrm{P}$ ), located at a suitable distance from the heme iron, can behave as an acid-base catalyst in hemoproteins $(16,20)$. Additionally, some unique P450 enzymes have been shown to function as peroxygenases by utilizing hydrogen peroxide as the oxygen donor with extremely high catalytic turnover rates compared to their normal monooxygenation reactions as well as the peroxide shunt reaction catalyzed by the common P450s $(12,21)$. A common structural feature about these proteins is that the distal side of the heme molecule is quite hydrophilic, which allows $\mathrm{H}_{2} \mathrm{O}_{2}$ to enter the active site followed by the formation of CpdI with the interaction of the carboxyl group of substrate and the Arg residue located near the heme (12).

In contrast, P450cam possesses a very hydrophobic distal environment that lacks the distal machinery of the peroxidases mentioned above. Therefore, to probe the roles of the nonpolar amino acids in the distal helix and to make an efficient peroxygenase, we substituted a distal residue with a hydrophilic histidine which is highly conserved in other 
heme peroxidases. In the distal pocket of $\mathrm{P} 450 \mathrm{cam}$, camphor is bound in a specific orientation above the heme iron, with a hydrogen bond between the camphor carbonyl and the phenol side chain of Tyr96 as well as numerous non-covalent contacts with active site residues (22). Val247 is one of the neighboring residues in the distal pocket that have hydrophobic contact with the substrate camphor (23). In light of its proximity to the heme prosthetic group, it served as a logical choice for our target (Fig. 2.1). The substitution of Val247 with a histidine was anticipated to provide a functional nitrogen from the imidazole group of His247 that could act as an acid-base catalyst via the peroxide-shunt pathway to facilitate the generation of Cpd I that affords substrate oxidation.

Various spectroscopic techniques were utilized in the present work for the structural and functional characterizations of the P450cam V247H mutant. In addition to electronic absorption spectroscopy, resonance Raman spectroscopy is well established as a highly sensitive probe of the active-site structure of heme proteins by providing detailed structural information on iron spin and ligation states. Circular dichroism was performed to examine the secondary and tertiary structural changes of the mutant. Proton nuclear magnetic resonance spectroscopy, one of the most powerful methods for high resolution structural characterization of paramagnetic metalloproteins, was also utilized to characterize the active site structure of the cyanide complexes of the wild type and mutant proteins. 

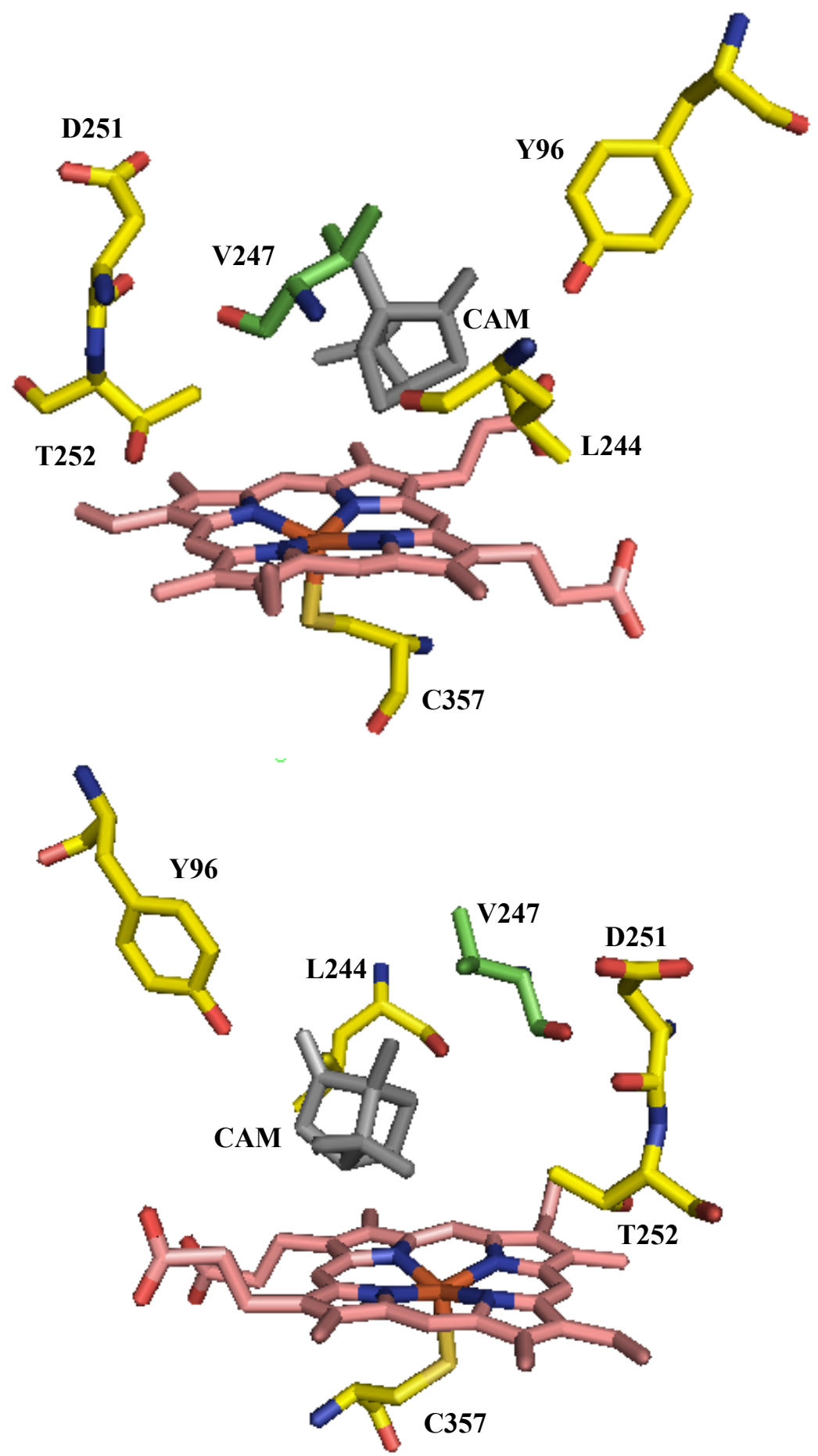

Figure 2.1 The active site structure of camphor-bound cytochrome P450cam: the heme (salmon), camphor (gray) and some selected residues including distal V247 (green) in P450cam are shown. The figures were generated from the crystal data (PDB code 2CPP) using PYMOL (22). 
Additionally, redox potential measurements of the protein were performed to show the effect of the amino acid substitution on the redox property of the heme iron.

\subsection{EXPERIMENTAL PROCEDURES}

\subsubsection{Materials}

The PfuUltra High-Fidelity DNA polymerase used for site-directed mutagenesis was from Stratagene and the primers for the mutation were synthesized by Eurofins MWG Operon (Huntsville, AL). Dithiothreitol (DTT), carbon monoxide, hydrogen peroxide, 2, 2'-Azino-bis (3-Ethylbenzthiazoline-6-Sulfonic Acid), and $\delta$ - aminolevulinic acid were purchased from Sigma-Aldrich (St. Louis, MO). Yeast extract, tryptone, glycerol, sodium chloride, sodium bromide, sodium acetate, sodium dithionite, potassium chloride, potassium phosphate, potassium cyanide, potassium hydroxide, EDTA, Tris-HCl, isopropyl- $\beta$-D-thiogalactopyranoside (IPTG), Ribonuclease A, Deoxyribonuclease I, phenylmethylsulfonyl fluoride (PMSF), lysozyme, hemin, DL-tyrosine and DL-camphor were obtained from Fisher Scientific (Pittsburgh, PA). DEAE sepharose fast flow and Sephadex G-75 were purchased from GE Healthcare (Piscataway, NJ). All chemicals were used without further purification. Choloperoxidase (CPO) from Caldariomyces fumago was prepared in our own lab with Rz value over 1.40. 


\subsubsection{Bacterial strains, plasmids and DNA manipulations}

The bacterial strains used were E. coli NovaBlue and E. coli Rosetta II (DE3) from Novagen (Gibbstown, NJ). The plasmid pUS200, constructed by Unger et al., contains a 1578-bp fragment of $P$. putida (ATCC 17453) P450cam plasmid fragment inserted into the PstI and HindIII sites of pEMBL8+ (24). To increase the protein yield, the P450cam gene was constructed into a T7-based expression vector, pET-30a $(+)$ from Novagen, at the sites of $\mathrm{NdeI}$ and HindIII by polymerase chain reactions and ligation reactions. Bacteria were grown in Luria-broth supplemented with antibiotic (kanamycin, $100 \mu \mathrm{g} / \mathrm{ml}$ ) for the DNA manipulation. Site-directed mutagenesis was performed by using this T7-based expression vector, pET-30a $(+)$. The V247H mutant of P450 cam was prepared with the 5'-sense oligonucleotide 5'- CTG TTA CTG CAT GGC GGC CTG GAT-3' coding for LLLEGGLD. The reconstituted plasmids of both wild type and V247H mutant were transformed into E. coli strain NovaBlue and verified by DNA sequencing.

\subsubsection{Protein Expression and Purification}

The wild type P450cam and mutant V247H were expressed in Escherichia coli strain Rosetta II (DE3). Cells were grown on terrific broth supplemented with $100 \mathrm{mg} / \mathrm{L}$ of kanamycin at $37{ }^{\circ} \mathrm{C}$ and induced with $1 \mathrm{mM}$ IPTG and $1 \mathrm{mM}$ camphor at $\mathrm{OD}_{600}$ around 0.8 , followed by further growth at $30^{\circ} \mathrm{C}$ for another $18 \mathrm{~h}$ prior to harvesting cells. As for

the mutant, the addition of $\delta$-aminolevulinic acid to the growth media is necessitated to facilitate holoenzyme production. 
Cells were lysed at $4^{\circ} \mathrm{C}$ in $50 \mathrm{mM}$ Tris- $\mathrm{HCl}$ buffer at $\mathrm{pH} 7.4$ containing $50 \mathrm{mM} \mathrm{KCl}$, $0.5 \mathrm{mM}$ DTT, $1 \mathrm{mM}$ camphor, $1 \mathrm{mM}$ EDTA, $200 \mu \mathrm{M}$ PMSF, $40 \mathrm{U} / \mathrm{ml}$ Dnase I, $3 \mathrm{U} / \mathrm{ml}$ Rnase A, and lysozyme. Following sonication and centrifugation, the lysate were concentrated by Amicon cell with 30,000 Da cut-off ultrafiltration membrane. The protein solution was applied onto a fast flow DEAE Sepharose column $(50 \mathrm{ml})$ equilibrated with $50 \mathrm{mM}$ Tris-HCl buffer (pH 7.4). After initial elution, the column was eluted with a linear $0-0.5 \mathrm{M} \mathrm{KCl}$ gradient in the same buffer. The red fractions containing the $\sim 45 \mathrm{kDa}$ proteins were pooled for further purification by size exclusion chromatography. Sephadex G-75 matrix $(500 \mathrm{ml})$ was used for the gel filtration and the buffer used was $100 \mathrm{mM}$ potassium phosphate containing $1 \mathrm{mM}$ camphor at $\mathrm{pH}$ 7.4. Substrate-free WT P450cam and its mutant were prepared by dialyzing the protein samples against phosphate buffer without camphor, followed by centrifugation with a microconcentrator (Centricon-30). Unless otherwise specified, all the steps in the protein purification were performed at $4{ }^{\circ} \mathrm{C}$.

The Rz values of pure wild-type P450cam and V247H mutant were over 1.4 and 1.3 respectively. The protein concentration was determined using the Soret extinction coefficient determined by the pyridine hemochrome method following the standard protocol $(25,26)$. The extinction coefficients for the camphor bound WT at $392 \mathrm{~nm}$ and mutant enzyme at $416 \mathrm{~nm}$ were 102 and $115 \mathrm{mM}^{-1} \mathrm{~cm}^{-1}$ respectively. These values were found to be 115 and $116 \mathrm{mM}^{-1} \mathrm{~cm}^{-1}$ for the camphor-free forms of the WT and mutant enzyme at $418 \mathrm{~nm}$. 


\subsubsection{Spectroscopic characterization}

Optical characterization of the wild type $\mathrm{P} 450_{\text {cam }}$ and its mutant was performed at room temperature on a Cary 300 spectrophotometer (Varian). The ferrous proteins were prepared by the addition of an excess amount of sodium dithionite and $\mathrm{CO}$ adducts were obtained by $\mathrm{CO}$ gas bubbling. The buffer used for the absorption measurements was $100 \mathrm{mM}$ potassium phosphate buffer with $1 \mathrm{mM}$ camphor at $\mathrm{pH}$ 7.4.

CD spectra were measured on a JASCO J-815 spectrometer at room temperature. The secondary-structure spectral region $(200-260 \mathrm{~nm})$ of the spectra was recorded in a quartz cuvette of $1 \mathrm{~mm}$ path length using about $2 \mu \mathrm{M}$ enzyme. As for the tertiary-structure spectral region $(260-650 \mathrm{~nm})$, it was recorded using about $20 \mu \mathrm{M}$ enzyme in a quartz cuvette of $1 \mathrm{~cm}$ path length with or without camphor.

For resonance Raman data collection, the wild-type P450 and V247H mutant were prepared in a septum-sealed, cylindrical quartz cell. Samples were reduced to the ferrous form by first flushing the sample with argon and then injecting a molar excess of buffered sodium dithionite solution. The rotating sample cell was irradiated with $4 \mathrm{~mW}$ of 413.1 nm laser light using a mixed krypton/argon ion laser (Spectra Physics, Beamlok 2060). The spectral acquisition time was $5 \mathrm{~min}$. The scattered light was collected at right angles to the incident beam and focused onto the entrance slit $(125 \mu \mathrm{m})$ of a $0.8 \mathrm{~m}$ spectrograph, where it was dispersed by a 600 groove/mm grating and detected by a liquid- $\mathrm{N}_{2}$-cooled CCD camera (Horiba-JY). Spectral calibration was performed against the lines of 
mercury. UV-vis spectra of each sample were collected before and after each RR measurement to ensure the sample integrity.

Proton nuclear magnetic resonance $\left({ }^{1} \mathrm{H}\right.$ NMR) spectra for the cyanide-bound proteins were recorded at $298 \mathrm{~K}$ on a Bruker Avance 600 (Ultrashield) spectrometer operating at a proton frequency of $599.97 \mathrm{MHz}$. Protein samples for NMR experiments were prepared in $\mathrm{D}_{2} \mathrm{O}$ buffer solutions containing $100 \mathrm{mM}$ potassium phosphate at $\mathrm{pH}$ 7.4, by at least four isotope exchanges of the protein solution in $\mathrm{H}_{2} \mathrm{O}$ with $\mathrm{D}_{2} \mathrm{O}$ buffer. The isotope exchanges were carried out in a microconcentrator (Centricon-30) at $4{ }^{\circ} \mathrm{C}$. All NMR samples contained more than $2 \mathrm{mM}$ protein and the cyanide adducts of the protein were prepared by the addition of a 10 -fold to 20 -fold molar excess of cyanide from a freshly made $1 \mathrm{M}$ stock solution of $\mathrm{KCN}$ in $99.9 \% \mathrm{D}_{2} \mathrm{O}$. The residual solvent signal was suppressed with presaturation during relaxation delay. Chemical shift values were referenced to the residual HDO signal at $4.76 \mathrm{ppm}$.

Phase-sensitive NOESY spectra for the cyanide-bound derivatives of P450cam and its $\mathrm{V} 247 \mathrm{H}$ mutant were acquired with mixing times ranging from 1.5 to $25 \mathrm{~ms}$. Typical NOESY spectra were collected with 256 experiments in the F1 dimension using the hypercomplex method (27). In general, 1600-2000 scans were accumulated for each F1 experiment, which was acquired with 4096 complex points in the F2 dimension over a spectral width of 27 or $60 \mathrm{kHz}$. The residual solvent signal in all NOESY experiments was suppressed using a 200-ms presaturation with a weak decoupler power. 
All the spectroscopic experiments were done with freshly prepared samples in $100 \mathrm{mM}$ potassium phosphate buffer $(\mathrm{pH} 7.4)$.

\subsubsection{Redox measurements}

The electrochemical measurements of wild type P450cam and V247H mutant were performed with a $\mathrm{CHI} 660$ electrochemical analyzer (CH Instruments) at room temperature by using a conventional three electrode cell, with a glassy carbon (GC) electrode as the working electrode, platinum wire as counter electrode and $\mathrm{Ag} / \mathrm{AgCl}$ electrode as the reference electrode. The carbon electrode has been modified with single-walled carbon nanotubes (SWCNT). The preparation of the SWCNTs was carried out as reported (28) and SWCNT suspension was cast onto the electrode and dried under infrared lamp. The SWCNT modified electrode is electrochemically activated by cyclical scanning between -1.0 and $+1.5 \mathrm{~V}$ at a scan rate of $1 \mathrm{~V} / \mathrm{sec}$ for 2 minutes to improve the sensitivity of the electrode. The redox potentials of P450camV247H mutant and the wild type protein were obtained and normalized with the reference to NHE.

\subsubsection{Peroxidase activity}

The peroxidation activity of the $\mathrm{V} 247 \mathrm{H}$ mutant was measured by using 2,2'-azino-bis-3-ethy-benzthiazoline-6-sulfonic acid (ABTS) as the electron donor and hydrogen peroxide as the sole oxidant (29). The reaction was initiated by the addition of $\mathrm{H}_{2} \mathrm{O}_{2}(3.3 \mathrm{mM})$ into the reaction solution of enzyme $(2 \mu \mathrm{M})$ and ABTS $(9.1 \mathrm{mM})$ in

$100 \mathrm{mM}$ potassium buffer from $\mathrm{pH} 5.0$ to $\mathrm{pH}$ 7.4. Wild type P450cam and 
chloroperoxidase were also examined at $\mathrm{pH}$ 5.0. Chloroperoxidase from Caldariomyces fumago was prepared in our own lab, and the $\mathrm{Rz}$ value of the protein used in this assay was over 1.4. The peroxidase activity was monitored at $405 \mathrm{~nm}$, and the initial rate of oxidized ABTS product formation was used to calculate the activity.

\subsection{RESULTS}

\subsubsection{UV-visible Spectroscopy study}

The UV-vis spectra for the ferric, ferrous, and ferrous-CO forms of V247H mutant were obtained to examine the coordination structure of the heme iron both in the absence and presence of camphor (Fig. 2.2A and 2.2B). The substrate-free V247H exhibited essentially identical spectra to that of the wild type enzyme. However, the camphor binding was perturbed in this mutant as compared to that in wild type according to the optical characterizations. Instead of having the Soret band shifted from $418 \mathrm{~nm}$ to $392 \mathrm{~nm}$ upon camphor binding, which is characteristic of the conversion of low-spin to high-spin Fe (III), the mutant only displayed a $2 \mathrm{~nm}$ blue shift from $418 \mathrm{~nm}$ to $416 \mathrm{~nm}$. Therefore, the substrate-bound $\mathrm{V} 247 \mathrm{H}$ mutant remained predominantly in the six-coordinate, low-spin ferric iron state, similarly as reported in the G248E mutant of cytochrome P450cam (30). The shape of the Soret band of camphor-bound V247H was also different from that of the camphor-free protein, with less symmetry and a less prominent $\delta$-band 
A

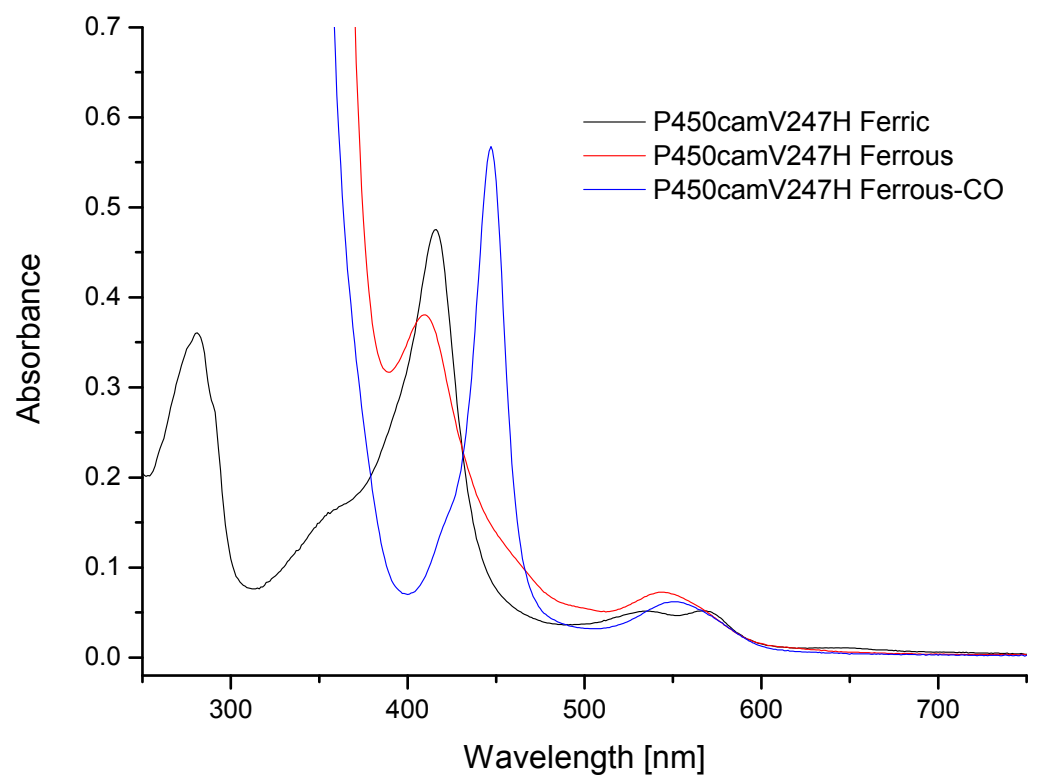

B

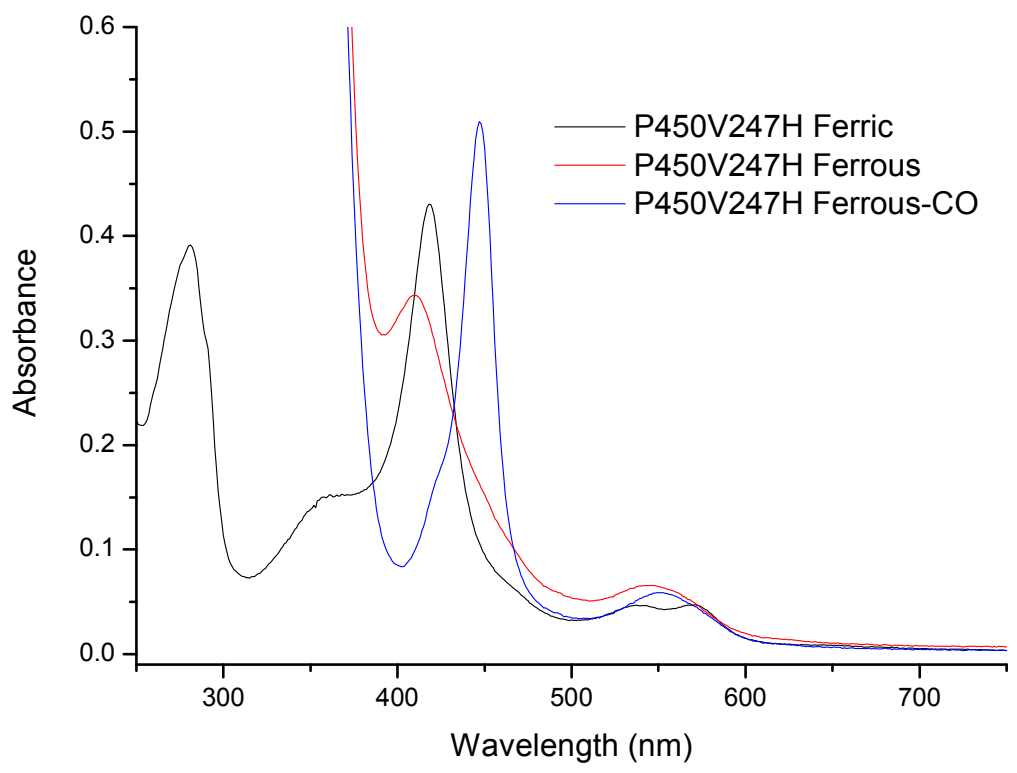

Figure 2.2. UV-vis spectra of $P 450$ camV247H in various oxidation states: (A) camphor-free and (B) camphor-bound forms in 100mM phosphate buffer ( $\mathrm{pH} 7.4)$ at room temperature. 
feature at around $360 \mathrm{~nm}$, which is an indication of a sixth water ligand bound to the heme iron. Otherwise, there were only very small differences in the visible absorption bands (Table 2.1).

Table 2.1. Wavelength and extinction coefficients of absorption maxima for wild type and V247H mutant of cytochrome P450cam

\begin{tabular}{lllll}
\hline Protein & Oxidation state & Soret $(\mathrm{nm})$ & $\delta$-band $(\mathrm{nm})$ & Visible $(\mathrm{nm})$ \\
\hline WTP450 & $\mathrm{Fe}^{3+}$ & $418(392)$ & 360 & 535,569 \\
(camphor-free) & $\mathrm{Fe}^{2+}$ & 408 & 540 \\
& $\mathrm{Fe}^{2+}-\mathrm{CO}$ & 446 & 550 \\
\hline V247H & $\mathrm{Fe}^{3+}$ & $418(\varepsilon=116)$ & 361 & 537,569 \\
camphor-free & $\mathrm{Fe}^{2+}$ & 409 & 540 \\
\hline $\mathrm{Fe}^{2+}-\mathrm{CO}$ & 447 & 548 \\
V247H & $\mathrm{Fe}^{3+}$ & $416(\varepsilon=115)$ & 355 & 534,569 \\
camphor-bound & $\mathrm{Fe}^{2+}$ & 409 & 542 \\
\hline
\end{tabular}

It is worth mentioning that for both camphor-free and camphor-bound samples, the ferrous complexes displayed the Soret maximum initially at approximately $412 \mathrm{~nm}$ upon reduction, but the addition of more sodium dithionite or mixing for a longer time period led to the Soret band shifting to $409 \mathrm{~nm}$. That could be an indication of the changed (most 
likely increased) electron density on the thiolate in the reduced state (31). The CO-bound form of $\mathrm{V} 247 \mathrm{H}$ showed a Soret maximum at $447 \mathrm{~nm}$, which supports the presence of an intact iron-sulfur coordination in this mutant (32). In addition, the ligand binding activity of the V247H showed very similar results compared with that of wild type. For example, the $\mathrm{KCN}$ binding of $\mathrm{V} 247 \mathrm{H}$ mutant displayed about a $24 \mathrm{~nm}$ red shift of the Soret band to $440 \mathrm{~nm}$ (Fig. 2.3), almost identical to that of the WT P450cam (33).

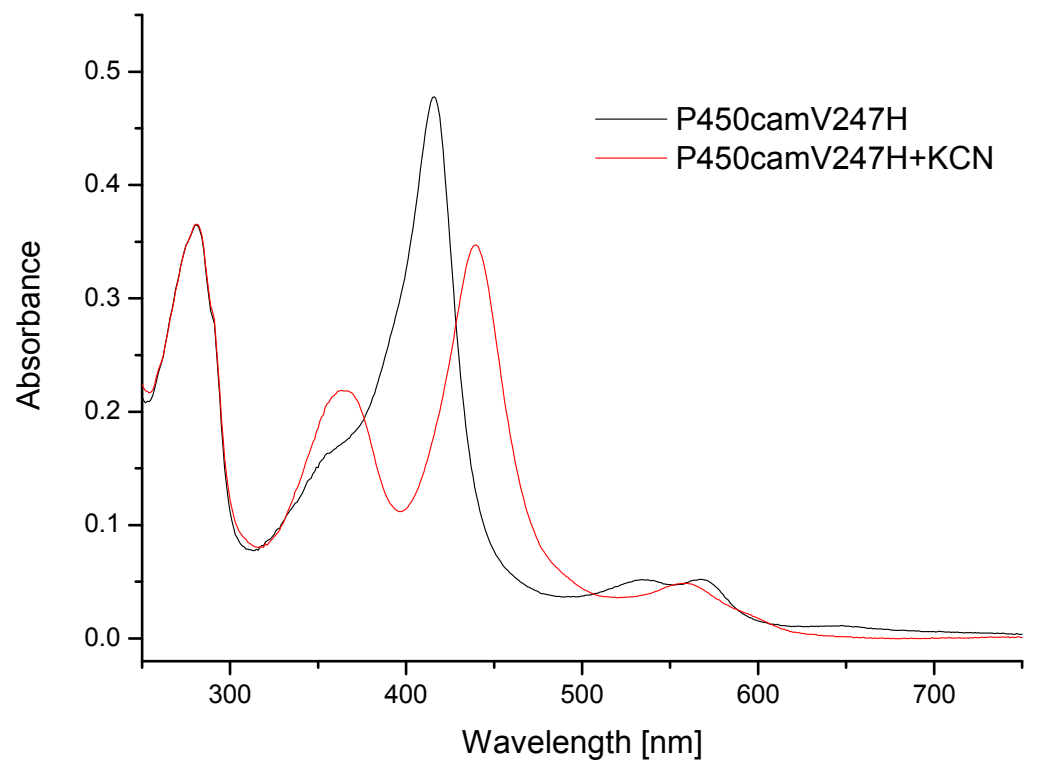

Figure 2.3. UV spectra of $\mathrm{P} 450$ camV247H and its $\mathrm{KCN}$ bound complex. The enzyme concentration employed was $\sim 4 \mu \mathrm{M}$, and $10 \mu 1$ of $1 \mathrm{M} \mathrm{KCN}$ was added to the enzyme.

\subsubsection{Circular Dichrosim spectroscopy study}

Multi-wavelength CD studies of the wild type and V247H mutant P450cam were carried out to investigate the effect of the distal residue substitution on the conformational 

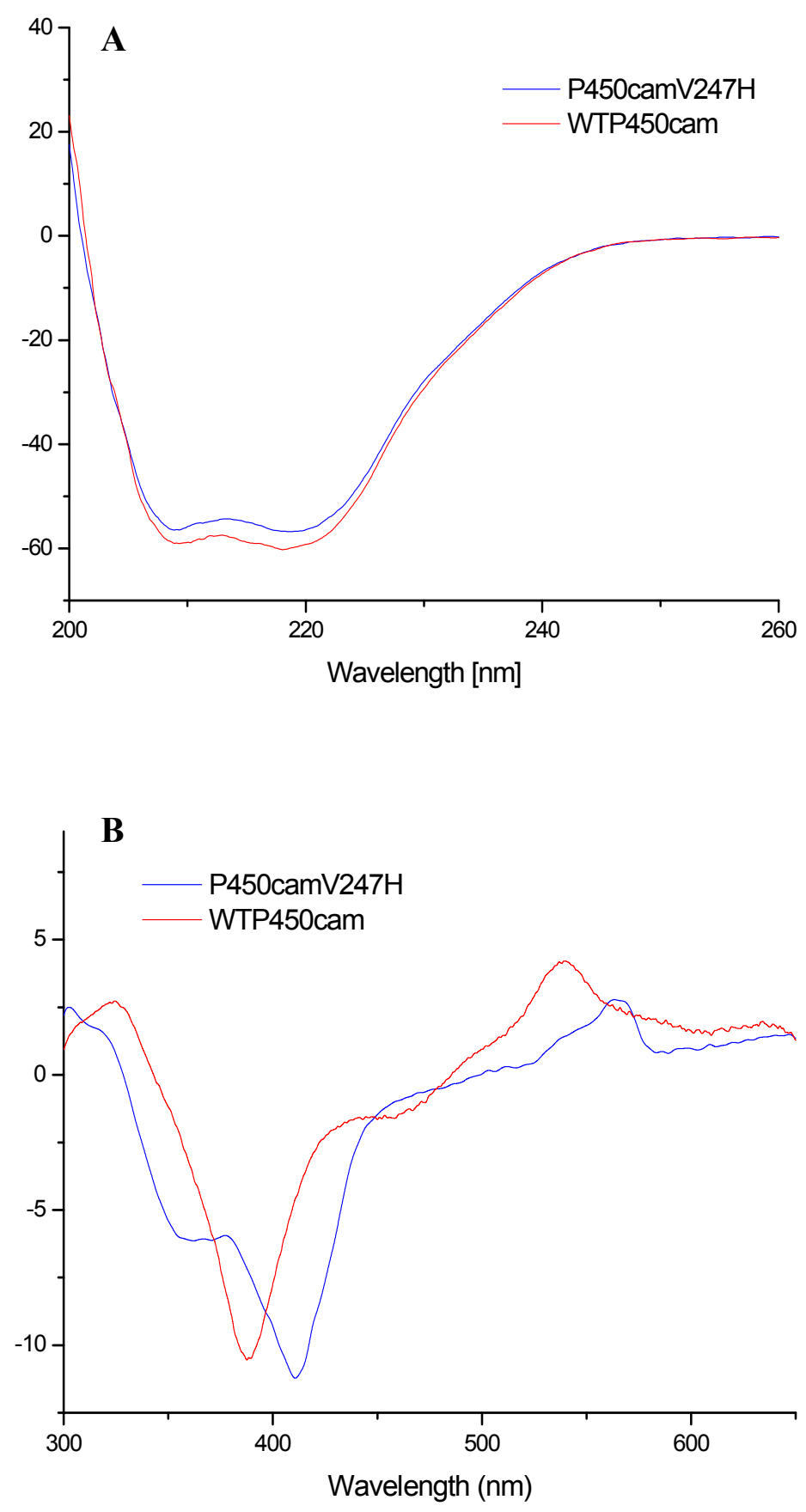

Figure 2.4. Far-UV CD (A) and visible CD (B) spectra of substrate-bound forms of the WT and V247H mutant of cytochrome P450cam. 
properties of the enzyme. As shown in Fig. 2.4 A, the far-UV CD bands from 200-260 nm, which provide information on the secondary-structural features of the proteins, showed no significant difference between the wild type and the mutant, suggesting that the mutation of the valine residue had no detectable effect on the secondary structure of the protein. However, in the near-UV-visible CD region $(300-650 \mathrm{~nm})$, the camphor-bound V247H mutant displayed rather different spectra in its ferric form from those of the wild type (Fig. 2.4 B). This was consistent with the result of optical absorption measurement that exhibited similar properties with the substrate-free wild type protein.

\subsubsection{Resonance Raman Spectroscopy study}

To examine the heme environment and the iron spin and ligation state of wild type P450cam and V247H mutant, we obtained resonance Raman spectra in the absence and presence of camphor. For the camphor-free ferric species, both wild type and V247H mutant displayed a strong oxidation state marker band $v_{4}$ at around $1370 \mathrm{~cm}^{-1}$ typical of ferric $\left(\mathrm{Fe}^{3+}\right)$ heme (Fig. 2.5A). The heme core-size marker bands $v_{3}$ and $v_{2}$ appeared at approximately 1500 and $1580 \mathrm{~cm}^{-1}$, respectively for the camphor-free samples, indicating a six-coordinate (6C), low-spin (LS) heme iron (34-36). For the camphor-bound proteins, the V247H mutant showed a different pattern from the wild type. Whereas the wild type displayed the $v_{3}$ and $v_{2}$ bands at 1486 and $1568 \mathrm{~cm}^{-1}$, indicative of five-coordinate (5C), high-spin (HS) heme iron component, the V247H displayed peaks at 1499 and $1581 \mathrm{~cm}^{-1}$, consistent with a 6C-LS complex, similar to the camphor-free samples. The RR spectra of 
ferrous $\mathrm{P} 450 \mathrm{cam}$ and $\mathrm{V} 247 \mathrm{H}$ with camphor bound showed a $v_{4}$ band at $1344 \mathrm{~cm}^{-1}$, consistent with thiolate proximal ligation (Fig. 2.5B) (37). The $v_{3}$ and $v_{2}$ band positions of these two samples at $1465 \mathrm{~cm}^{-1}$ and $1562 \mathrm{~cm}^{-1}$ confirmed that the ferrous forms of both wild type and mutant complexes were 5C-HS. However, the ferrous forms of substrate-free wild type and mutant proteins displayed a different spectral pattern from the camphor-bound forms. Most notably, the $v_{4}$ band appeared at 1358 instead of 1344 $\mathrm{cm}^{-1}$, indicating a loss of or modulation of the proximal thiolate ligation. However, for the wild type, there was a $1344 \mathrm{~cm}^{-1}$ shoulder of the $v_{4}$ band, indicating that a weak population of thiolate proximal ligand was retained. 
A. Ferric

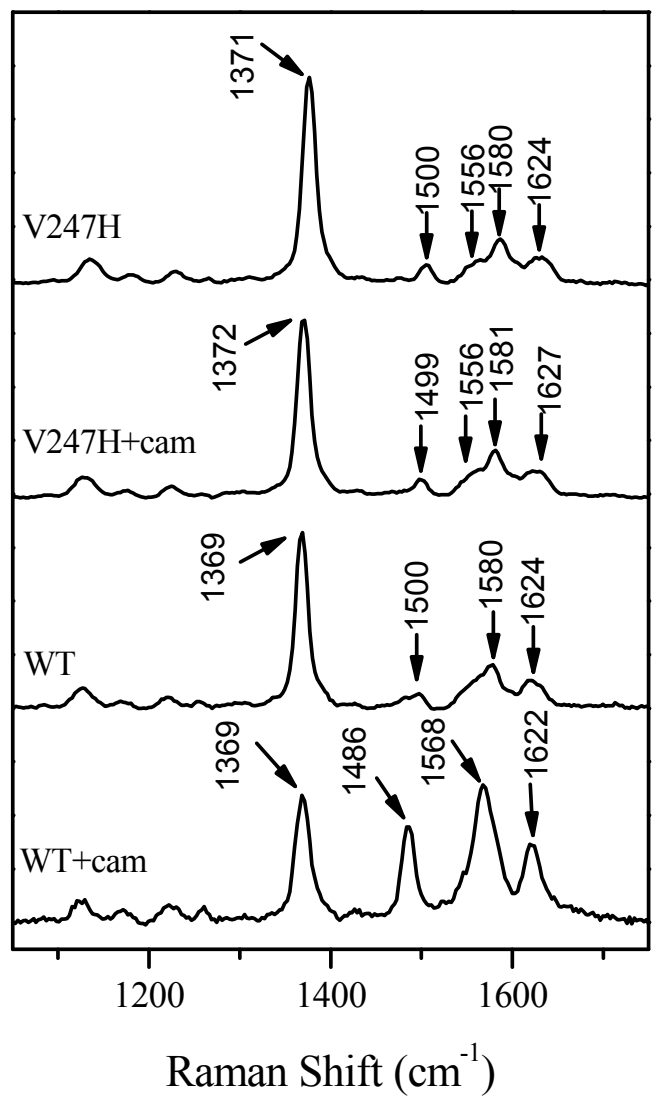

B. Ferrous

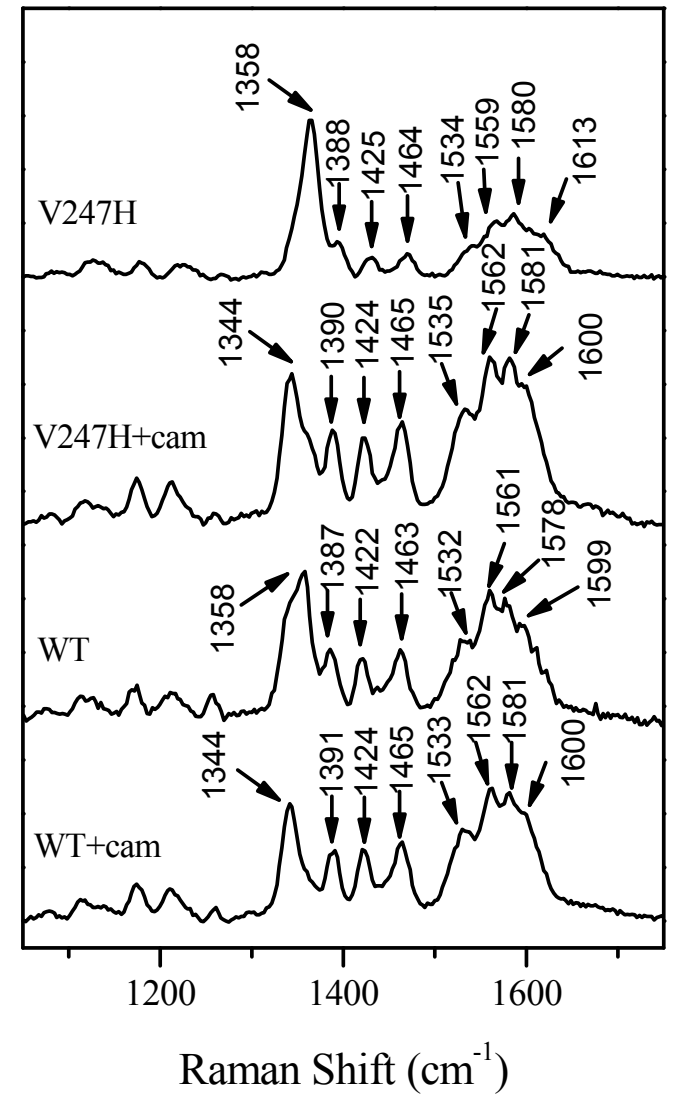

Figure 2.5. Resonance Raman spectra of (A) ferric and (B) ferrous wild-type P450 and $\mathrm{P} 450 \mathrm{~V} 247 \mathrm{H}$ mutant with and without camphor binding. The excitation wavelength $\left(\lambda_{\mathrm{ex}}\right)$ was $413.1 \mathrm{~nm}$. The spectra of $\mathrm{P} 450$ and $\mathrm{V} 247 \mathrm{H}$ samples in the presence and absence of $1 \mathrm{mM}$ camphor are shown.

\subsubsection{NMR Spectroscopic study}

Figure 2.6 showed the 1D proton NMR spectra of ferric low spin, cyanide-bound complexes of both wild type and V247H mutant of P450cam in the presence of camphor. The wild type P450cam exhibited almost identical results as repored in the literature, with two broad, fast relaxing, strongly hyperfine-shifted peaks at $33.1 \mathrm{ppm}$ and $-21.4 \mathrm{ppm}$ respectively, indicative of the $\beta$ protons from the coordinated Cys357 (38). 


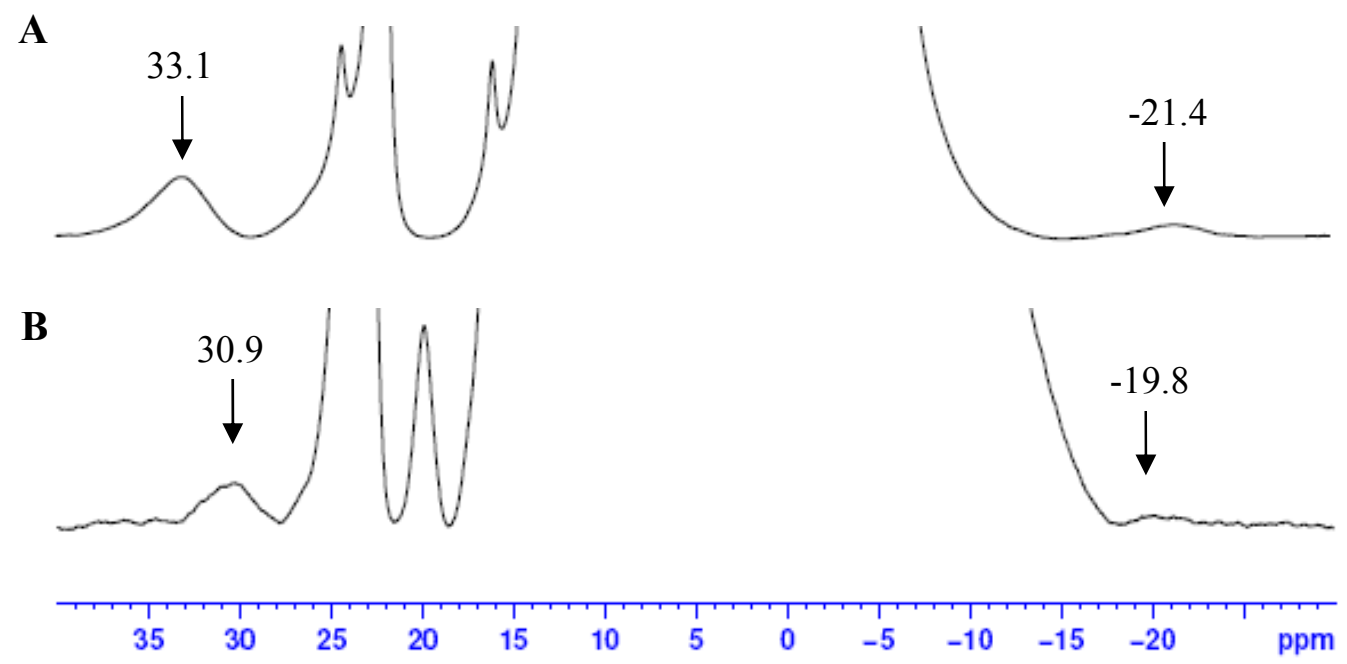

Figure 2.6 600-MHz ${ }^{1} \mathrm{H}$ Nuclear Magnetic Resonance spectra of the low spin cyanide complexes of (A) wild-type P450 and (B) V247H mutant. The spectra are collected at $298 \mathrm{~K}$ in $0.1 \mathrm{M}$ phosphate buffer at $\mathrm{pH} 7.4$.

Chloroperoxidase also shows a similar spectral pattern with that of P450cam with two hyperfine-shifted peaks at 38.3 and $-20.7 \mathrm{ppm}$, the characteristic features of protons in close proximity to the heme iron from Cys29 (27). The P450cam V247H mutant (Fig. 2.6 (B)) displayed similar results, but with a slight shift compared with the wild type, which could be the result of conformational change caused by the substitution of the distal Val247. Nevertheless, the pair of peaks at $30.9 \mathrm{ppm}$ and $-19.8 \mathrm{ppm}$ suggested the thiolate ligation in the $\mathrm{V} 247 \mathrm{H}$ mutant was remained.

\subsubsection{Electrochemistry study}

Various reconstituted hemoproteins with modified heme or associated residues have been measured to analyze the factors that control their functions (39-41). However, the 
A
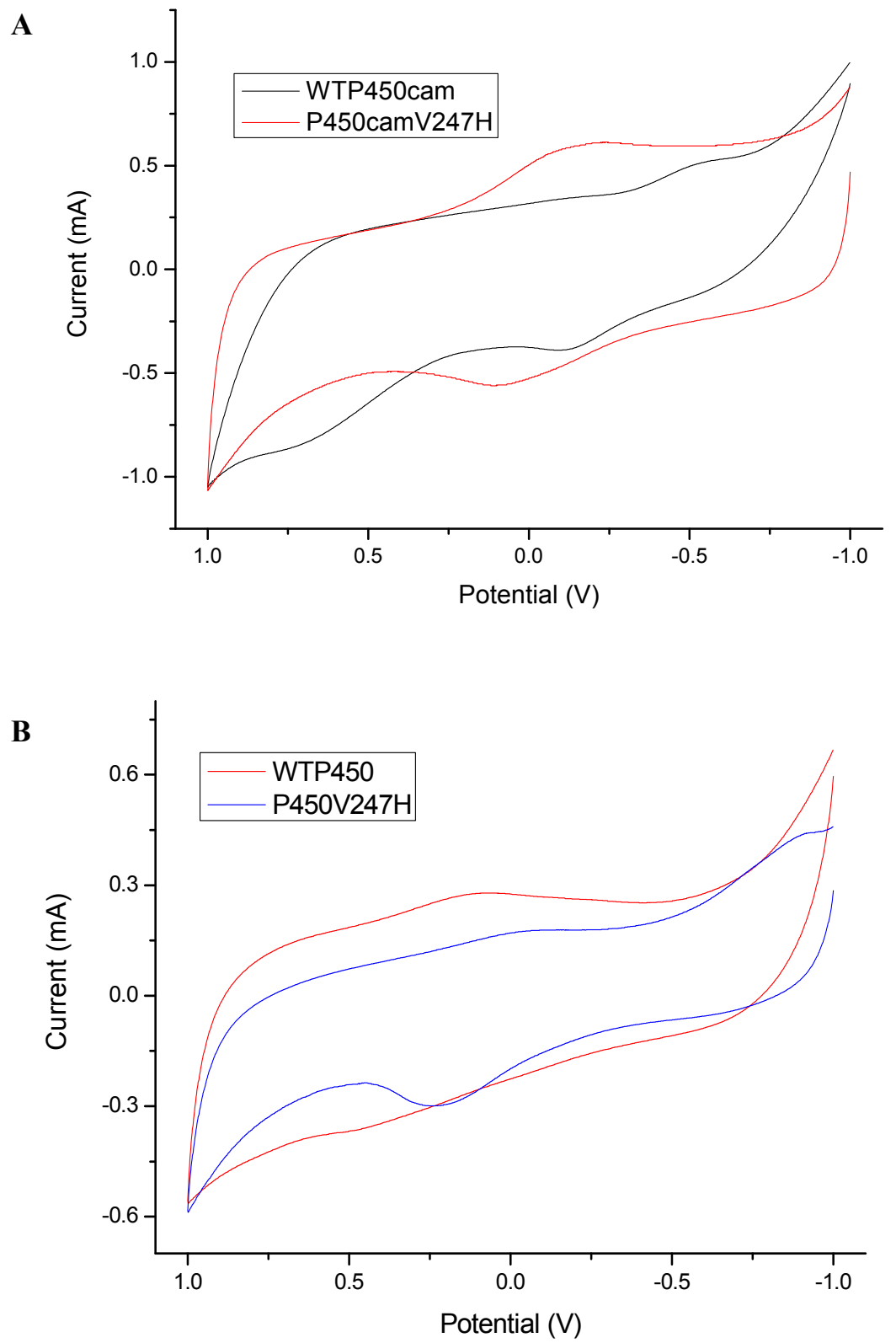

Figure 2.7 Cyclic voltammograms of WT P450cam and V247H mutant on a glass carbon electrode modified with SWNTs in the presence (A) and absence (B) of substrate camphor. Voltammograms were recorded between -1.0 and $+1.5 \mathrm{~V}$ at the scan rate of $1 \mathrm{~V} / \mathrm{sec}$ in $100 \mathrm{mM}$ phosphate buffer (pH7.4). 
direct determination of the redox properties of heme proteins is often rendered difficult due to the strong adsorption of the protein onto electrode surface. The use of SWNT-coated carbon electrodes virtually eliminates this problem.

The reduction potentials for wild type P450cam were measured to be -304 and -135 $\mathrm{mV}$ in the absence and presence of camphor, respectively, both of which were in good agreement with the reported values (42). While the formal potential for the camphor-free V247H mutant was observed, to be much greater than the wild type, as high as $+256 \mathrm{mV}$, upon substrate binding the value was decreased by about $50 \mathrm{mV}$ to $+206 \mathrm{mV}$ (Fig. 2.7). This suggests that the substitution of the distal valine residue with a histidine induced a positive shift of the $\mathrm{Fe}^{3+} / \mathrm{Fe}^{2+}$ redox couple that might be attributed to the conformational change in the distal pocket.

\subsubsection{ABTS assay}

As shown in Fig. 2.8, the V247H mutant exhibited significant peroxidase activity compared with the wild type protein, and its reactivity was enhanced dramatically as $\mathrm{pH}$ increased. The highest peroxidation reactivity of the V247H mutant was observed at $\mathrm{pH}$ 7.4, which is the physiological optimum $\mathrm{pH}$ for P450cam. It displayed even higher peroxidase activity than $\mathrm{CPO}$, and about 700 -fold increase compared with the wild type P450cam at $\mathrm{pH} 5.0$. 
A
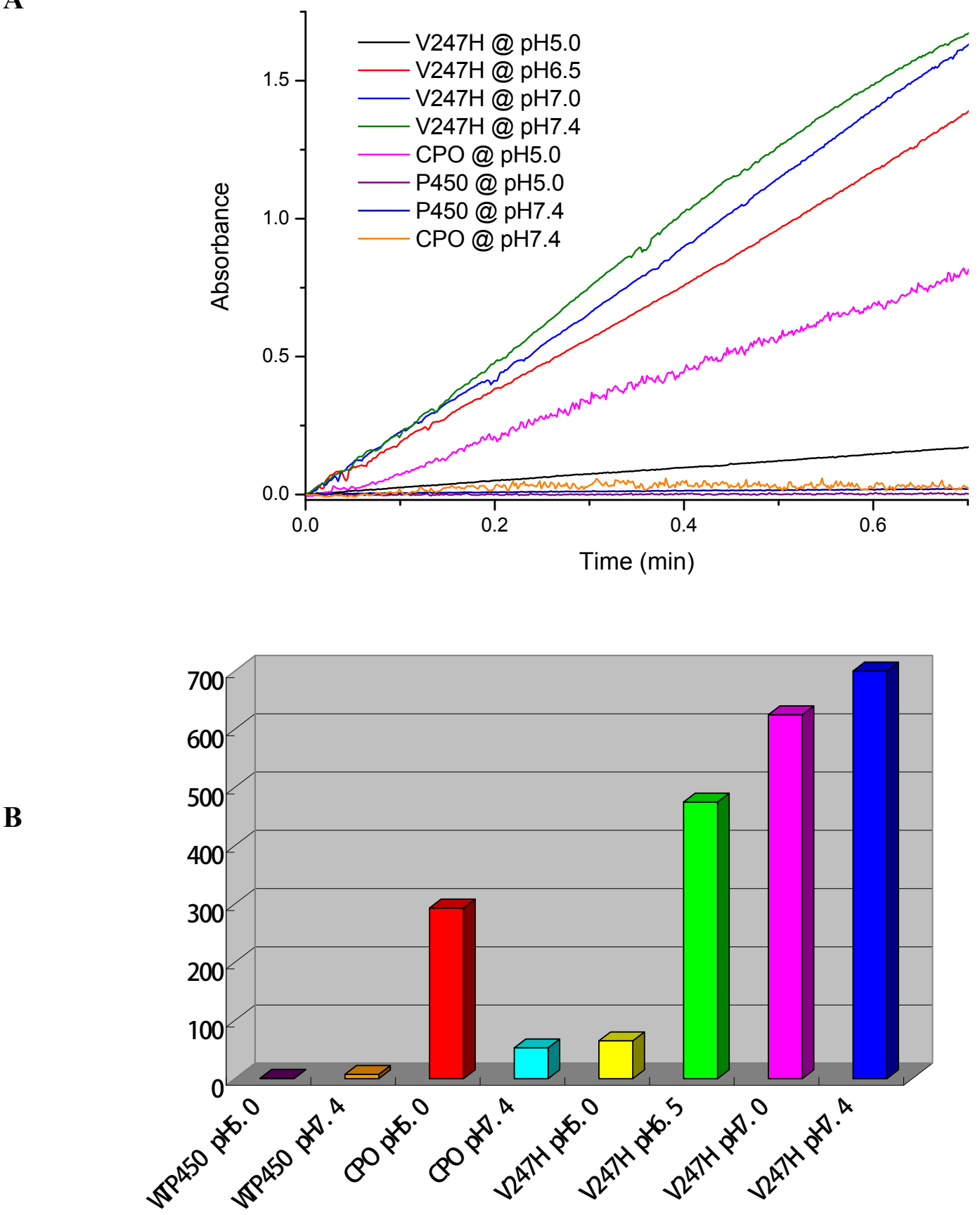

Figure 2.8 ABTS assay of cytochrome P450cam, V247H mutant and CPO at different $\mathrm{pH}$ values. The peroxidase activity was monitored at $405 \mathrm{~nm}$ in the presence of ABTS $(9.1 \mathrm{mM})$, enzyme $(2 \mu \mathrm{M})$, and $\mathrm{H}_{2} \mathrm{O}_{2}(3.3 \mathrm{mM})$ in $100 \mathrm{mM}$ potassium phosphate buffer. The initial slope of the absorbance was used to calculate the peroxidase activity. 


\subsection{DISCUSSION}

Though P450s are potentially significant enzymes for catalyzing the insertion of oxygen into unactivated $\mathrm{C}-\mathrm{H}$ bonds, the practical application of cytochromes $\mathrm{P} 450$ as industrial biocatalysts has been greatly limited by the requirement of expensive cofactors and partner proteins. In this study, we converted the P450cam into an efficient peroxidase without the aid of any auxiliary elements by replacing the significant distal amino acid Val247 with a histidine residue. A distal His is found in many heme peroxidases and thus V247H mimics their distal environment.

The ABTS assay, which serves as a typical method to examine the peroxidase activity, was performed to test the mutant. We showed that after substitution of Val247 with a histidine, the mutant gained significantly higher peroxidase activity driven by $\mathrm{H}_{2} \mathrm{O}_{2}$. Although in many heme peroxidases the distal histidine plays a key role in the formation of CpdI, a nearby arginine residue (also highly conserved) has also been suggested to cooperate with the histidine, thus playing an important role in assisting the catalytic cycle $(17,43,44)$. Considering the possible contribution that a distal arginine might make to the peroxidase activity of $\mathrm{V} 247 \mathrm{H}$, we also replaced the nearby amino acid L244 in the distal helix with an arginine. However this double mutant L244R/V247H did not show higher peroxidase activity than the single mutant under the same conditions of study (data not shown). Previously, the proximal Cys357 was substituted with a 
histidine to mimic heme peroxidases, but the $\mathrm{V} 247 \mathrm{H} / \mathrm{C} 357 \mathrm{H}$ mutant did not exhibit better “peroxidase" activity compared with V247H alone (45).

The $\mathrm{V} 247 \mathrm{H}$ mutant of P450cam was characterized by various spectroscopic techniques. UV-vis spectra showed that the resting state of $\mathrm{V} 247 \mathrm{H}$ mutant without camphor is a six-coordinate, low spin ferric heme complex, similar to wild type P450cam, which has cysteinate and $\mathrm{H}_{2} \mathrm{O}$ serving as the axial ligands. However, in addition to the wild type, V247H remained 6C-LS with addition of camphor. This suggests that the camphor-bound $\mathrm{V} 247 \mathrm{H}$ mutant still remains predominantly in the six-coordinated, low-spin ferric iron state. This indicates that the camphor enters or binds to the heme prosthetic group differently due to the possible conformational changes caused by the replacement of Val247. The substituted bulkier histidine residue might sterically hinder the entrance of the substrate by having the imidazole group oriented in the direction of the heme iron. This was further confirmed with the CD spectroscopic study. Nevertheless, the characteristic Soret band of the ferrous-CO complex of $\mathrm{V} 247 \mathrm{H}$ appeared at around 450nm, suggesting that the $\mathrm{V} 247 \mathrm{H}$ mutant maintains the proximal heme-thiolate coordination. The reduced form of the mutant possesses a Soret maximum at $412 \mathrm{~nm}$ upon initial reduction, which probably implies increased electron density on the thiolate in the reduced state $(46)$.

Resonance Raman spectroscopy has proven to be a powerful probe to investigate the heme environment, especially the coordination, spin state and axial ligations of the heme. 
In this study, both substrate-free and substrate-bound wild type and mutant proteins were examined (Fig. 3.4). Overall the spectra of P450cam with camphor in different oxidation states agree well with previous $\mathrm{RR}$ results $(47,48)$. The RR results of the ferric forms of the mutant protein in the presence and absence of camphor are also consistent with the UV-Vis absorption data discussed earlier.

The $v_{4}$ band in the $\mathrm{RR}$ spectrum of $5 \mathrm{C}-\mathrm{Fe}^{2+}$ heme complexes is a useful diagnostic marker of the identity of the proximal ligand. RR studies of a wide variety of heme proteins over the years have established that $5 \mathrm{C}-\mathrm{Fe}^{2+}$ heme complexes with histidine (imidazole) as proximal ligand produce a $v_{4}$ band in the $1350-1360 \mathrm{~cm}^{-1}$ region whereas those with a cysteine (thiolate) proximal ligand display a $v_{4}$ band in the $1340-1348 \mathrm{~cm}^{-1}$ region $(37,49)$. The ferrous forms of camphor-bound wild type P450cam and V247H mutant display a $v_{4}$ band in the range for $5 \mathrm{C}$ thiolate proximal ligation. Although the $\mathrm{UV}$ absorption spectrum of the ferrous-CO complex of camphor-free V247H indicated that the thiolate proximal ligand is preserved, the RR spectrum of the ferrous form does not indicate the same based on the previously established signatures. Thus, the $v_{4}$ band is located at $1358 \mathrm{~cm}^{-1}$, significantly higher than the expected $1340-1348 \mathrm{~cm}^{-1}$ range for proximal thiolate ligation (37). A $v_{4}$ peak at $1358 \mathrm{~cm}^{-1}$ was also observed for the camphor-free wild type protein, although in this case a shoulder was observed at 1344 $\mathrm{cm}^{-1}$. Evidently, in the absence of camphor, the proximal thiolate ligand is altered either by being fully replaced by a histidine ligand, or by being protonated or elongated. 
Replacement of the cysteine ligand by a histidine appears unlikely because the CD data did not indicate any significant conformational changes upon camphor binding (Fig. 3.4 (A)). Also, we substituted all of the histidine residues present in the distal helix with either glycine or alanine and this species exhibited the same RR spectroscopic features (see Chapter III). Therefore it is likely that the proximal cysteine in the present case is protonated rather than replaced by another proximal residue. Indeed, Sabat et al. recently showed that protonation of the proximal cysteine ligand in iNOS leads to similar RR spectroscopic features as has been traditionally ascribed to a proximal histidine ligand $(50)$.

The electrochemistry of this mutant together with wild type P450cam has shown that the distal environment can regulate the redox potential of the heme iron. For heme-containing oxygenases and peroxidases, the "push-pull" mechanism has been proposed where the generation of Cpd I is facilitated by an anionic proximal ligand and/or positive polar distal residues (51). In peroxidases like horseradish peroxidase or cytochorme $c$ peroxidase, the "push" effect is provided by the proximal histidine and "pull" effect is mainly provided by the distal histidine that serves as a general acid-base catalyst to generate and stabilize the Cpd I $(16,52)$. For P450cam, since it lacks the distal residue that could promote the heterolytic O-O bond cleavage, the strong "push" effect from the proximal thiolate axial ligand is proposed to be responsive for the $\mathrm{O}-\mathrm{O}$ bond heterolysis. It has also been suggested that the total charge near the heme could be 
important for the heme redox potential. In the V247H mutant of P450cam, the redox potential was greatly increased, over $300 \mathrm{mV}$ higher compared with that of the wild type. The increased redox potential of the V247H mutant clearly indicated the enhanced "pull" effect because of the introduction of the distal hisitine residue which would increase the total charge near the heme prosthetic group and facilitate the generation of Cpd I.

In summary, we have shown that the substitution of Val247 in the distal helix with a hydrophilic histidine residue, which is highly conserved in many heme peroxidases, has successfully converted P450cam into an efficient self-sufficient $\mathrm{H}_{2} \mathrm{O}_{2}$-driven peroxidase, with the thiolate ligation intact in the proximal side.

\subsection{REFERENCES}

1. Guengerich, F. P. (1991) Reactions and Significance of Cytochrome P-450 Enzymes, Journal of Biological Chemistry 266, 10019-10022.

2. Ortiz de Montellano, P. R. (2005) Cytochrome P450: structure, mechanism, and biochemistry, 3 ed., Kluwer Academic/Plenum Publishers, New York.

3. Sigel, A., Sigel, H., and Sigel, R. K.O. (2007) The ubiquitous roles of cytochrome P450 proteins in Metal Ions in Life Sciences (Sigel, A., Sigel, H., and Sigel, R. K.O., Ed.), Wiley, Chichester.

4. Sono, M. R., M. P.; Coulter, E. D.; and Dawson, J. H. (1996) Heme-Containing Oxygenases, Chemical Reviews 96, 2841-2847.

5. Denisov, I. G., Makris, T. M., Sligar, S. G., and Schlichting, I. (2005) Structure and chemistry of P450, Chem. Review 105, 2253-2277. 
6. Dawson, J. H. (1988) Probing structure-function relations in heme-containing oxygenases and peroxidases, Science 240, 433-439.

7. Newcomb, M., Zhang, R., Chandrasena, R. E. P., Halgrimson, J. A., Horner, J. H., Makris, T. M., and Sligar, S. G. (2006) Cytochrome P450 Compound I, J. Am. Chem. Soc. 128, 4580-4581.

8. Joo, H., Lin, Z. L., and Arnold, F. H. (1999) Laboratory evolution of peroxide-mediated cytochrome P450 hydroxylation, Nature 399, 670-673.

9. Nordblom, G. D., White, R. E. and Coon, M. J. (1976) Studies on hydroperoxide-dependent substrate hydroxylation by purified liver microsomal cytochrome P450, Arch. Biochem. Biophys. 175, 524-533.

10. Hrycay, E. G., Gustafsson, J.-A., Ingelman-Sundberg, M. and Ernster, L. (1975) Sodium periodate, sodium chlorite, organic hydroperoxides and hydrogen peroxide as hydroxylating agents in steroid hydroxylation reactions catalyzed by partially purified cytochrome P450, Biochem. Biophys. Res. Commun. 66, 209-216.

11. Cirino, P. C., and Arnold, F. H. (2003) A self-sufficient peroxide-driven hydroxylation biocatalyst, Angew. Chem. Int. Ed. 42, 3299-3301.

12. Shoji, O., Fujishiro, T., Nakajima, H., Kim, M., Nagano, S., Shiro, Y., Watanabe, Y. (2007) Hydrogen Peroxide-Dependent Monooxygenations by Tricking the Substrate Recognition of Cytochrome P450BS $\beta$, Angew. Chem. Int. Ed. 46, 3656-3659.

13. Cirino, P. C., and Arnold, F. H. (2002) Regioselectivity and activity of cytochrome P450 BM-3 and mutant F87A in reactions driven by hydrogen peroxide, Advanced Synthesis \& Catalysis 344, 932-937.

14. Poulos, T. L., and Kraut, J. (1980) The Sterochemistry of Peroxidase Catalysis, J. Biol. Chem. 255, 8199-8205.

15. Davydov, R. M., Ledenev, A.N. (1981) Activation mechanism of molecular oxygen by cytochrome P-450, Biofizika 26, 1096. 
16. Tanaka, M., Ishimori, K., Mukai, M., Kitagawa, T., and Morishima, I. (1997) Catalytic activities and structural properties of horseradish peroxidase distal His42-> Glu or Gln mutant, Biochemistry 36, 9889-9898.

17. Howes, B. D., RodriguezLopez, J. N., Smith, A. T., and Smulevich, G. (1997) Mutation of distal residues of horseradish peroxidase: Influence on substrate binding and cavity properties, Biochemistry 36, 1532-1543.

18. Newmyer, S. L., and Demontellano, P. R. O. (1995) Horseradish-peroxidase His-42 -> Ala, His-42 ->Val, and Phe-41 ->Ala mutants - Histidine Catalysis and Control of Substrate Access to the Heme Iron, J. Biol. Chem. 270, 19430-19438.

19. Erman, J. E., Vitello, L. B., Miller, M. A., Shaw, A., Brown, K. A., and Kraut, J. (1993) Histidine 52 is a critical residue for rapid formation of cytochrome c peroxidase compound I, Biochemistry 32, 9798-9806.

20. Poulos, T. L., and Kraut, J. (1980) THE STEREOCHEMISTRY OF PEROXIDASE CATALYSIS, J. Biol. Chem. 255, 8199-8205.

21. Matsunaga, I., and Shir, Y. (2004) Peroxide-utilizing biocatalysts: structural and functional diversity of heme-containing enzymes, Current Opinion in Chemical Biology 8, 127-132.

22. Poulos, T. L., Finzel, B. C., and Howard, A. J. (1987) HIGH-RESOLUTION CRYSTAL-STRUCTURE OF CYTOCHROME-P450CAM, Journal of Molecular Biology 195, 687-700.

23. Poulos, T. L., Finzel, B. C., Gunsalus, I. C., Wagner, G. C., and Kraut, J. (1985) THE 2.6-A CRYSTAL-STRUCTURE OF PSEUDOMONAS-PUTIDA CYTOCHROME-P-450, Journal of Biological Chemistry 260, 6122-6130.

24. Unger, B. P., Gunsalus, I. C., Sligar, S. G. (1986) Nucleotide-sequence of the Pseudomonas-putida Cytochrome P450-cam Gene and Its Expression in Escherichia-coli, J. Biol. Chem. 261, 1158-1163.

25. Paul, K. G., Theorell, H., Akesson, A. (1953) The Molar Light Absorption of Pyridine Ferroprotoporphyrin (Pyridine Haemochromogen), Acta Chem. Scand. 7, 1284-1287. 
26. Berry, E. A., and Trumpower, B. L. (1987) Simultaneous Determination of Hemes-a, Hemes-b, and Hemes-c from Pryidine Hemochrome Spectra, Analytical Biochemistry 161, 1-15.

27. Wang, X., Tachikawa, H., Yi, X., Manoj, K. M., and Hager, L. P. (2003) Two-dimensional NMR Study of the Heme Active Site Structure of Chloroperoxidase, J. Biol. Chem. 278, 7765-7774.

28. Alwarappan, S., Prabhulkar, S., Durygin, A., and Li, C. . (2009) The effect of electrochemical pretreatment on the sensing performance of single walled carbon nanotubes, J. Nanosci. Nanotech. 9, 2991-2996.

29. Yi, X. W., Mroczko, M., Manoj, K. M., Wang, X. T., and Hager, L. P. (1999) Replacement of the proximal heme thiolate ligand in chloroperoxidase with a histidine residue, Proc. Natl. Acad. Sci. U.S.A. 96, 12412-12417.

30. Limburg, J., LeBrun, L. A., and de Montellano, P. R. O. (2005) The P450(cam) G248E mutant covalently binds its prosthetic heme group, Biochemistry 44, 4091-4099.

31. Suzuki, N., Higuchi, T., Urano, Y., Kikuchi, K., Uekusa, H., Ohashi, Y., Uchida, T., Kitagawa, T., and Nagano, T. (1999) Novel Iron Porphyrin-Alkanethiolate Complex with Intramolecular NH...S Hydrogen Bond: Synthesis, Spectroscopy, and Reactivity, J. Am. Chem. Soc. 121, 11571-11572.

32. Gunsalus, I. C., Wagner, G. C. (1978) Bacterial P-450cam methylene monooxygenase components: Cytochrome $\mathrm{m}$, putidaredoxin, and putidaredoxin reductase, Methods Enzymol. 52, 166-188.

33. Dawson, J. H., Andersson, L. A. and Sono, M. (1982) Spectroscopic Investigations of Ferric Cytochrome P-450-CAM Ligand Complexes, J. Biol. Chem. 257, 3606-3617.

34. Spiro, T. G., Stong, J. D., and Stein, P. (1979) Porphyrin core expansion and doming in heme proteins. New evidence from resonance Raman spectra of six-coordinate high-spin iron(III) hemes, J. Am. Chem. Soc. 101, 2648-2655.

35. Andersson, L. A., Mylrajan, M., Sullivan, E. P., Jr., and Strauss, S. H. (1989) J. Biol. Chem. 264, 19099-19102. 
36. Lou, B. S., Snyder, J. K., Marshall, P., Wang, J. S., Wu, G., Kulmacz, R. J., Tsai, A. L., and Wang, J. (2000) Biochemistry 39, 12424-12434.

37. Anzenbacher, P., Evangelista-Kirkup, R., Schenkman, J., Spiro, T. G. (1989) Influence of Thiolate Ligation on the Heme Electronic-Structure in Microsomal Cytochrome-P450 and Model Compounds - Resonance Raman-Spectroscopic Evidence, Inorganic Chemistry 28, 4491-4495.

38. Wakasugi, K., Ishimori, K., and Morishima, I. (1996) NMR studies of recombinant cytochrome P450cam mutants, Biochimie 78, 763-770.

39. Li, C., Taniguchi, I., Mulchandani, A. . (2009) Redox properties of engineered ruthenium myoglobin, Bioelectrochemistry 75, 182-188.

40. Li, C., Nishiyama, K. and Taniguchi, I. (2000) Electrochemical and spectroelectrochemical studies on cobalt myoglobin, Electriochim. Acta. 45, 2883-2888.

41. Li, C. Z., Taniguchi, I., Mulchandani, A. (2009) Redox properties of engineered ruthenium myoglobin, Bioelectrochemistry 75, 182-188.

42. Yoshioka, S., Takahashi, S., Ishimori, K., Morishima, I. (2000) Roles of the axial push effect in cytochrome P450cam studied with the site-directed mutagenesis at the heme proximal site, J. Inorg. Biochem. 81, 141-151.

43. Schiodt, C. B., Veitch, N. C., and Welinder, K. G. (2007) Roles of distal arginine in activity and stability of Coprinus cinereus peroxidase elucidated by kinetic and NMR analysis of the Arg51Gln, -Asn, -Leu, and -Lys mutants, Journal of Inorganic Biochemistry 101, 336-347.

44. Bujons, J., Dikiy, A., Ferrer, J. C., Banci, L., and Mauk, A. G. (1997) Charge reversal of a critical active-site residue of cytochrome-c peroxidase Characterization of the Arg48->Glu variant, European Journal of Biochemistry $243,72-84$.

45. Auclair, K., Moenne-Loccoz, P., Ortiz de Montellano, P. R. (2001) Roles of the Proximal Heme Thiolate Ligand in Cytochrome P450cam, J. Am. Chem. Soc. 123, 4877-4885. 
46. Yoshioka, S., Tosha, T., Takahashi, S., Ishimori, K., Hori, H., and Morishima, I. (2002) Roles of the Proximal Hydrogen Bonding Network in Cytochrome P450cam-Catalyzed Oxygenation, J. Am. Chem. Soc. 124, 14571-14579.

47. Champion, P. M., Gunsalus, I. C., and Wagner, G. C. (1978) Resonance Raman Investigations of Cytochrome P450cam from Pseudomonas-putida, J. Am. Chem. Soc. $100,3743-3751$.

48. Wells, A. V., Li, P. S., Champion, P. M., Martinis, S. A., and Sligar, S. G. (1992) Resonance Raman Investigations of Escherichia-coli Expressed Pseudomonas-putida Cytochrome-P450 and Cytochrome P420, Biochemistry 31, 4384-4393.

49. Wang, J., Caughey, W. S., Rousseau, D. L. (1996) Resonance Raman Scattering: a Probe of Heme Protein-bound Nitric Oxide., in Methods in Nitric Oxide Research (Feelisch, M., Stamler, J., Ed.), pp 427-454, J. Wiley, Chichester ; New York.

50. Sabat, J., Stuehr, D. J., Yeh, S. R., and Rousseau, D. L. (2009) Characterization of the Proximal Ligand in the P420 Form of Inducible Nitric Oxide Synthase, Journal of the American Chemical Society 131, 12186-12192.

51. Takahashi, S., Wang, J., Rousseau, D. L., Ishikawa, K., Yoshida, T., Host, J. R., and Ikeda-Saitot, M. (1994) Heme-Heme Oxygenase Complex, J. Biol. Chem. 269, 1010-1014.

52. Newmyer, S. L., and Ortiz de Montellano, P. R. (1996) Rescue of the Catalytic Activity of an H42A Mutant of Horseradish Peroxidase by Exogenous Imidazoles, J. Biol. Chem. 271, 14891-14896. 


\section{CHAPTER III. ENIGNEERING CYTOCHROME P450 INTO P420 WITH CATALYTIC ACTIVITIES}

\subsection{SUMMARY}

In an effort to engineer cytochrome P450cam into a self-sufficient biocatalyst, key structural components within the heme active site of P450cam and chloroperoxidase (CPO) were evaluated and the P450cam L246K mutant was constructed. L246K works as a self-sufficient peroxidase demonstrating the importance of polarity of the distal heme environment in regulating the catalytic property of P450cam. The similar UV-vis and resonance Raman (RR) spectra of ferric L246K and substrate-free ferric P450cam support a hexacoordinate, low-spin heme with thiolate ligation in this form of the mutant. Surprisingly, the reduced L246K and its CO complex display spectral properties similar to those of the inactivated species of P450cam (P420), suggesting the proximal ligand of L246K was changed or modified during reduction and CO binding. Contrary to what has been generally thought, formation of a myoglobin-like heme structure in L246K via histidine coordination is ruled out by the expression of a P450cam mutant (L246K-3H, $\mathrm{H} 352 \mathrm{G} / \mathrm{H} 355 \mathrm{G} / \mathrm{H} 361 \mathrm{~A}$ ) where all three histidines located in the vicinity of the proximal ligand helix were replaced by glycine or alanine. On the basis of the spectral similarities and functional differences among L246K, the native P450cam, and the inactive P420, it is proposed that upon reduction and $\mathrm{CO}$ binding, the proximal cysteine ligand of P420 becomes protonated due to harsh conditions employed in its preparation while the proximal ligand of L246K retains the parent protein's thiolate character but with a longer Fe-S bond due to conformational changes induced by the mutation. 


\subsection{INTRODUCTION}

The cytochromes P450 are a ubiquitous class of heme-containing monooxygenases named for their characteristic optical absorption maximum near $450 \mathrm{~nm}$ upon reduction in the presence of carbon monoxide (1). They catalyze a variety of difficult chemical transformations with high specificity and selectivity under physiological conditions, making them attractive candidates for large-scale production of fine chemicals and pharmaceuticals that are difficult to synthesize with standard organic chemistry (2-4).

Cytochrome P450cam (CYP101) from Pseudomonas putida catalyzes the hydroxylation of camphor and has been extensively studied as the prototype of P450 monooxygenases because of its solubility, availability and extensive mechanistic and structural characterizations. It was the first to be characterized by crystallographic techniques among P450 enzymes (5). Previous study has shown that the resting state of P450cam is a predominately low spin Fe (III) porphyrin with water cluster occupying the sixth coordination position, upon substrate binding the water is replaced by the substrate camphor and the complex becomes high spin followed by reduction to Fe (II) and subsequent binding of $\mathrm{O}_{2}(6)$.

The P450s and chloroperoxidase (CPO) from Caldariomyces fumago are distinct from most heme proteins in possessing a deprotonated cysteine residue at the proximal side serving as the axial ligand to the heme iron (Fig. 3.1) (7-9). The role of axial-thiolate ligation in P450s has been widely studied and the thiolate ligand is suggested to serve as 
a strong internal electron donor, facilitating the heterolytic cleavage of the dioxygen bond to generate the activated iron (IV)-oxo species known as Cpd I $(10,11)$. Also the presence of the cysteinate thiolate proximal heme iron ligand is believed to be responsible for the red-shift of the Soret absorption band at 450nm. Although P450s and CPO share similar metal binding sites, their heme environments are quite different in terms of polarity (12). The active site of P450 is highly hydrophobic in nature with the lack of obvious acid-base catalytic residues in close proximity to the oxygen binding pocket, while $\mathrm{CPO}$ has evolved a more polar distal heme pocket analogous to that of the hydro-peroxidases (13). Another dissimilarity between P450cam and CPO is that CPO only needs hydrogen peroxide to be catalytically active, while P450cam requires dioxygen, cofactor NADH and two electron-transfer proteins-putidaredoxin reductase and putidaredoxin which are known as the "physiological partners" (14). These requirements for activity are believed to be the major factors that hinder the application of P450s in biotechnological processes. The goal of this study is to understand the structure-functional relationships in hemoproteins and to investigate the feasibility of converting P450cam to an active catalyst without cofactors and explore the correlation and possible conversion between P450s and peroxidases (such as CPO).

Most P450 enzymes would undergo a universal denaturing transition to a stable but inactive species known as $\mathrm{P} 420 \mathrm{~s}$ because the absorption maximum of the resulting $\mathrm{Fe}^{\mathrm{II}}-\mathrm{CO}$ complex is at $420 \mathrm{~nm}$ rather than at $450 \mathrm{~nm}$ as is characteristic of P450 (15). 
Cytochrome P420 could be formed from all known types of P450 by numerous chemical and physical techniques, such as incubation in organic solvents (16), exposure to high temperature, pressure $(17,18)$, neutral salts $(19)$, or to very low and high $\mathrm{pH}$ buffer $(20)$. P420 can sometimes, depending on how it is prepared, be converted back to P450 by incubation with thiols, spermine, dimethyl sulfoxide, polyols, and native substrates ( 16 , 22-25) . Therefore, knowledge about the structural relationship between the inactive P420 and the native P450 is of paramount importance to our understanding of the catalytic activity and the nature of the putative transient intermediates involved in the catalytic cycle of P450 enzymes. Furthermore, the structural alterations that occur during the conversion of P450 to P420 will not only shed light on the conformational modification in many hemoprotein variants with thiolate axial ligands, but also greatly help us in the rational design of self sufficient P450 mutants with enhanced activity and stability.

In fact, there are some other P420 forms of the heme-thiolate proteins besides P450. As a member of P450 family, inducible nitric oxide synthase (iNOS) possesses a negatively charged thiolate ligand from a deprotonated cysteine residue proximal to the heme iron (26). Like other P450 proteins, the reduced iNOS exhibits the absorption Soret band at around 450nm upon CO binding (iNOSP450), which can spontaneously convert to an inactive species like "P420" (iNOSP420) with the Soret band at around 420nm (27-30). The spectroscopic studies have shown that the iNOSP420 species is inactivated by protonation of the proximal thiolate ligand to a neutral thiol, instead of by ligand 
A

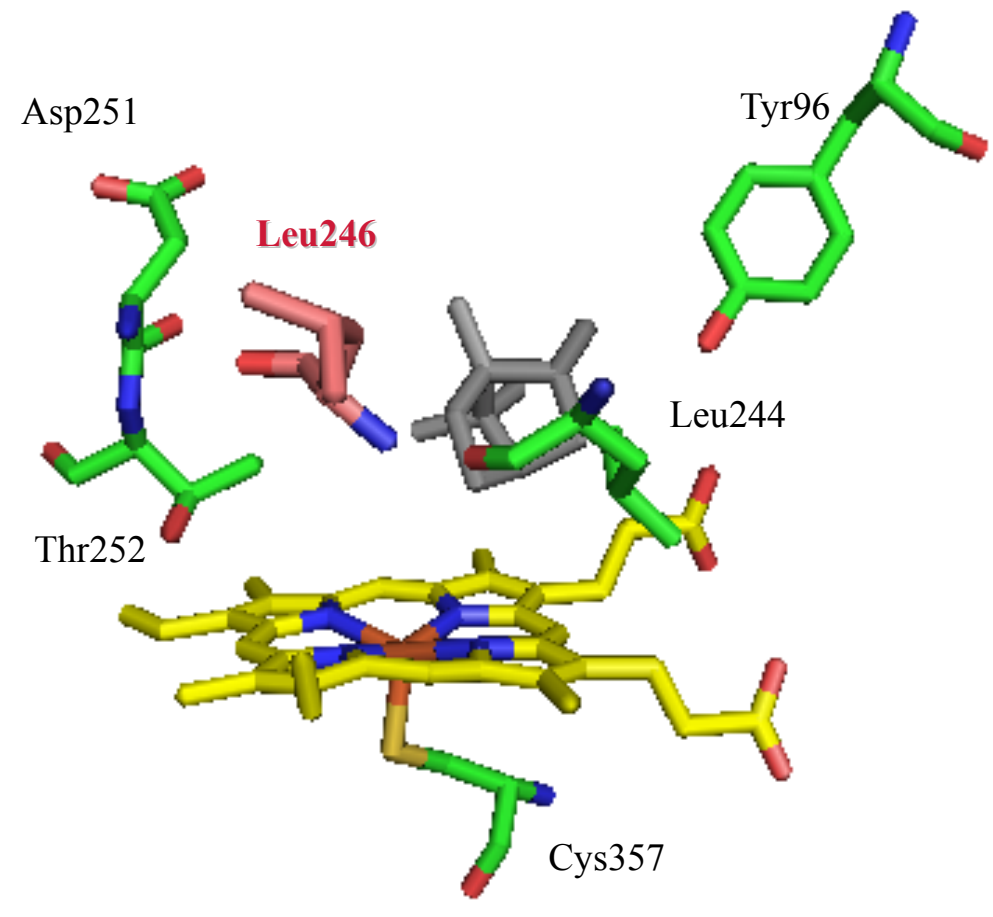

B
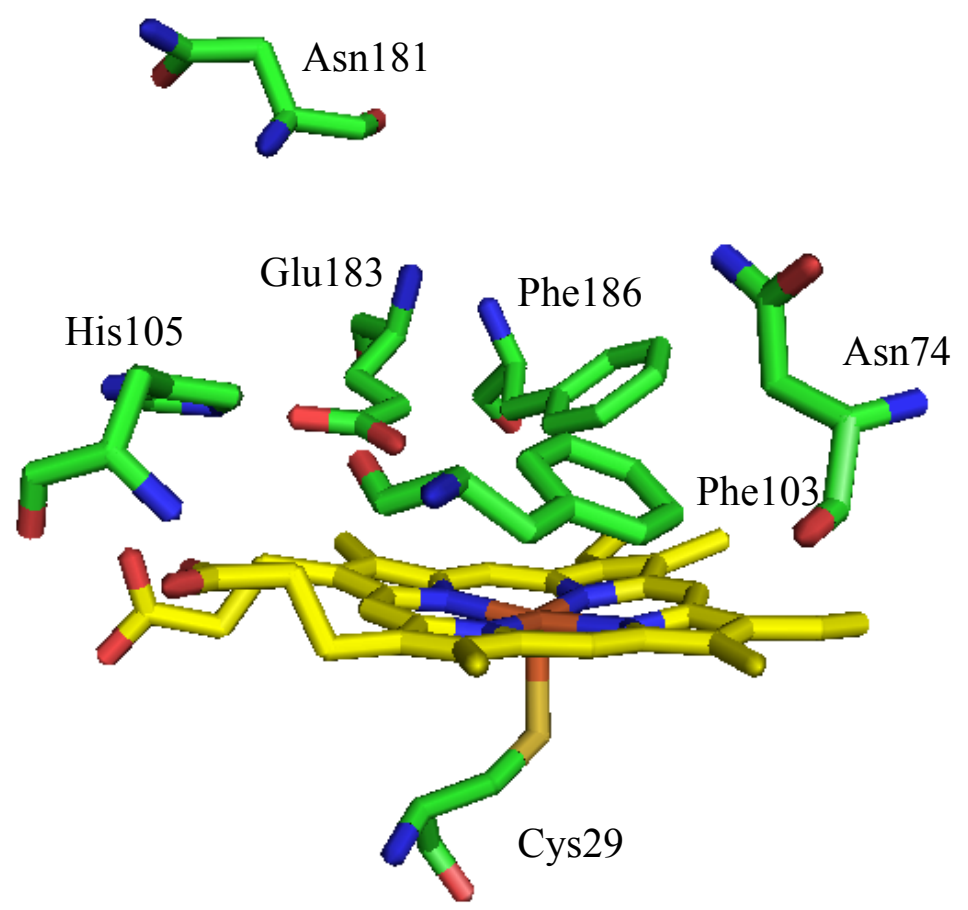

Figure 3.1 Heme environmental structure of ferric cytochrome P450cam (A) and chloroperoxidase (CPO: B): the heme (yellow), camphor (gray) and some selected residues including distal Leu246 (salmon) in P450cam are shown. The figures were generated from the crystal data (PDB code 2CPP and 1CPO) using PYMOL $(5,21)$. 
switching (e.g. to a histidine) (31). The iNOSP450 to iNOSP420 transition is controlled by the conformational state of the enzyme. In addition chloroperoxidase, under alkaline conditions, could also undergo a denaturating transition to an inactive species that has a spectrum essentially identical to that of cytochrome P420, and is named C420 (32). Although the conversion of $\mathrm{CPO}$ to $\mathrm{C} 420$ can be stopped at any point by lowering the $\mathrm{pH}$, it is not likely to convert $\mathrm{C} 420$ back to native $\mathrm{CPO}$ by adjusting the complex to lower $\mathrm{pH}$ values or by adding natural sulfhydryl compounds such as mercaptides (33). The heme iron oxidation state has been suggested to influence the transition to $\mathrm{C} 420$ which triggers the conformational change. In ferric C420, the proximal ligand C29 appears at least partially retained in a thiolate/imidazole coordination sphere. However the ferrous C420 loses the thiolate ligand and has a bis-histidine coordination structure instead. Upon $\mathrm{CO}$ binding, one of the axial histidine liagnds remains trans to the carbon monoxide (32).

Although many physical and chemical methods have been employed to investigate the structural features of P420 and its counterparts in iNOS and CPO, the structural relationship between $\mathrm{P} 450$ and P420 remains controversial (22, 34-36). Postulation concerning the structure of P420 has been focused on the nature of the proximal heme iron ligand. In particular, it has been suggested that a conformational change results in replacement of the thiolate ligand by histidine or exogenous ligand, yielding a myoglobin (Mb)-like heme coordination structure (36-38). Alternatively, theoretical calculations and recent studies with $\mathrm{Mb}$ H93G cavity mutant have indicated that the proximal thiolate may 
be protonated, resulting in a weaker thiol ligand $(39,40)$, or that the Fe-S bond might be weakened by lengthening (41). Unfortunately, there is little experimental evidence in the literature to distinguish these hypotheses $(35,42)$. Therefore, it is desirable to make a P450 variant that does not need harsh physical and chemical treatment but displays P420-like spectral features.

In an effort to engineer P450cam into a self sufficient catalyst, we designed several mutants focused on modulating the polarity of the distal heme environment. As predicted by the structural comparison of $\mathrm{P} 450$ with most heme peroxidases, introducing polar residues to the heme distal environment indeed improved the catalytic efficiency of P450 dramatically. First, multiple mutations were carried out in P450cam to replace the hydrophobic amino acids in the distal side of the heme group with polar amino acids. The P450 variant was named as $\mathrm{P} 450 \mathrm{H}$ with the substitutions of L244K, L245K, L246K, L250K, T252H, V253A and V254R. The spectroscopic studies of $\mathrm{P} 450 \mathrm{H}$ showed P420-like properties in its ferrous and ferrous-CO complexes. To elucidate which one or ones of the nonpolar residues in the distal pocket induced such transition, single mutation and multiple mutations were performed. It turned out that the L246K mutant of P450 was the first example that demonstrated typical spectral features of P420s but acted as a self-sufficient catalyst. Through the process, the introduction of polar residues to the heme distal environment indeed improved the catalytic efficiency of P450 dramatically. More significantly, structural and biochemical studies of this P420-P450 hybrid allowed 
us to discern the nature of the proximal heme iron ligand in the active P450 and the inactive $\mathrm{P} 420$ species.

\subsection{EXPERIMENTAL PROCEDURES}

\subsubsection{Site-directed mutagenesis and protein expression.}

The pUS200 plasmid used in this work, containing a 1578-bp CAM plasmid fragment of Pseudomonas putida (ATCC 17453), was subcloned into the PstI and HindIII sites of vector pEMBL8+. To increase the protein yield, the P450cam gene was constructed into pET-30a (+), a high copy number vector, at the sites of NdeI and HindIII by polymerase chain reactions and ligation reactions. The $\mathrm{L} 246 \mathrm{~K}$ mutant of $\mathrm{P} 450_{\text {cam }}$ was

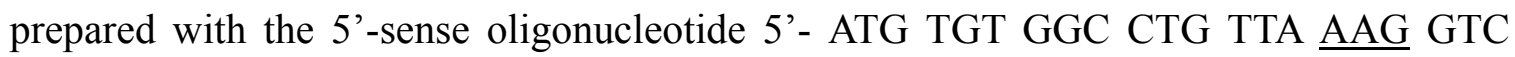
GGC coding for MCGLLKVG. The L246K 3H mutant was obtained based on the L246K mutant with the 5'-sense oligonucleotide 5'- TTT GGC GGT GGC AGC GGT CTG TGC

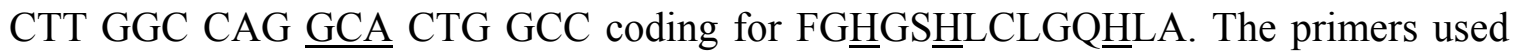
for $\mathrm{P} 450 \mathrm{H}$, and other mutants were listed in Appendix C. The reconstructed plasmids containing wild type P450cam and its $\mathrm{P} 450 \mathrm{H}, \mathrm{L} 246 \mathrm{~K}$ and L246K 3H mutants were transformed into E. coli strain NovaBlue and verified by DNA sequencing. Then the proteins were expressed in E. coli strain Rosetta II (DE3). Cells were grown on terrific broth supplemented with $100 \mathrm{mg} / \mathrm{L}$ of kanamycin at $37{ }^{\circ} \mathrm{C}$ and induced with $1 \mathrm{mM}$ IPTG and $1 \mathrm{mM}$ camphor at $\mathrm{OD}_{600}$ around 0.8 , followed by further growth at $30^{\circ} \mathrm{C}$ for another 
$18 \mathrm{~h}$ prior to harvesting cells. As for the mutants, the addition of $\delta$-aminolevulinic acid to the growth media is necessitated to facilitate holoenzyme production. The cells were then harvested and frozen at $-80^{\circ} \mathrm{C}$.

\subsubsection{Protein purification}

Cells were lysed at $4^{\circ} \mathrm{C}$ in $50 \mathrm{mM}$ Tris- $\mathrm{HCl}$ buffer at $\mathrm{pH} 7.4$ containing $50 \mathrm{mM} \mathrm{KCl}$, $0.5 \mathrm{mM}$ DTT, $1 \mathrm{mM}$ camphor (buffer A), $1 \mathrm{mM}$ EDTA, $200 \mu \mathrm{M}$ PMSF, $40 \mathrm{U} / \mathrm{ml}$ Dnase I, $3 \mathrm{U} / \mathrm{ml}$ Rnase A, and lysozyme. Following sonication and centrifugation, the lysed cells were concentrated by Amicon cell with 30,000 Da cut-off ultrafiltration membrane. The protein solution was first applied on a fast flow DEAE Sepharose column. After initial elution, the column was eluted with a linear $0-0.5 \mathrm{M} \mathrm{KCl}$ gradient in the same buffer. As for the L246K and L246K 3H mutants, they were expressed as apo-proteins. So the fractions containing the $\sim 45 \mathrm{kDa}$ proteins were pooled for heme reconstitution. Hemin was added to the apo-protein dropwise and stirred overnight (43). After heme reconstitution, the protein was again applied on the fast flow DEAE Sepharose column. The reddish fractions of correct molecular weight were pooled and concentrated using a Centriprep with an YM30 membrane for further purification by size exclusion chromatography. The buffer applied on the gel filtration was $0.1 \mathrm{M}$ potassium phosphate buffer at $\mathrm{pH} 7.4$. 
P420 was prepared as reported by adding $30 \%$ acetone and incubate at room temperature for 30 minutes (16). Unless otherwise specified, all the steps in the protein purification were performed at $4{ }^{\circ} \mathrm{C}$.

\subsubsection{Electronic absorption spectroscopy}

The UV-visible spectra of the P450cam mutants were recorded on a Varian Cary 300 Bio UV-visible spectrophotometer at room temperature. The ferrous proteins were prepared by the addition of excess amount of sodium dithionite and $\mathrm{CO}$ adducts were obtained by $\mathrm{CO}$ gas bubbling. The buffer used for the absorption measurements was $100 \mathrm{mM}$ potassium phosphate buffer at $\mathrm{pH} 7.4$.

\subsubsection{Resonance Raman spectroscopy}

For the resonance Raman data collection, the wild-type P450 and its mutant samples with concentration about $50 \mu \mathrm{M}$ were prepared in a septum-sealed, cylindrical quartz cell. Samples were reduced to the ferrous form by first flushing the sample with argon and then injecting a molar excess of buffered sodium dithionite solution. The rotating sample cell was irradiated with $4 \mathrm{~mW}$ of $413.1 \mathrm{~nm}$ laser light using a mixed krypton/argon ion laser. The spectral acquisition time was $5 \mathrm{~min}$. The scattered light was collected at right angles to the incident beam and focused onto the entrance slit $(125 \mu \mathrm{m})$ of a $0.8 \mathrm{~m}$ spectrograph, where it was dispersed by a 600 groove $/ \mathrm{mm}$ grating and detected by a liquid- $\mathrm{N}_{2}$-cooled CCD camera (Horiba-JY). Spectral calibration was performed against 
the lines of mercury. UV-vis spectra of each sample were collected before and after each RR measurement to ensure the sample integrity.

\subsubsection{Peroxidase activity}

2, 2'-azino-bis (3-ethylbenzthiazoline-6-sulfonic acid) (ABTS) was used as the substrate for the enzymatic assay of peroxidase activity of wild type P450cam, $\mathrm{P} 450 \mathrm{H}$, CPO, P420 and the P450cam L246K mutant protein. 9.1mM ABTS substrate solution was prepared in $50 \mathrm{mM}$ phosphate buffer at $\mathrm{pH} 5.0$. Hydrogen peroxide was added to initiate the reaction, and the increase of the absorbance was monitored at $405 \mathrm{~nm}$ for 2 min. The spectrophotometer cell holder was equipped with a magnetic stirrer and the incubation mixture was stirred throughout the reaction.

\subsection{RESULTS AND DISCUSSION}

\subsubsection{Optical properties and catalytic activity of $\mathrm{P} 450 \mathrm{H}$}

The P450H mutant of cytochrome P450cam, in which seven hydrophobic amino acids were replaced by polar residues, showed very different electronic absorption properties with those of the wild type in all oxidation states (Fig. 3.2). Especially, the ferrous- $\mathrm{CO}$ complex of the $\mathrm{P} 450 \mathrm{H}$ mutant displayed the absorption maximum at $420 \mathrm{~nm}$ instead of 450nm which is characteristic of P450. The blue shift of the spectrum could be caused by the change of polarity in the distal pocket and some distinct conformational alteration in the active site. To examine whether the $\mathrm{P} 450 \mathrm{H}$ mutant with increased 


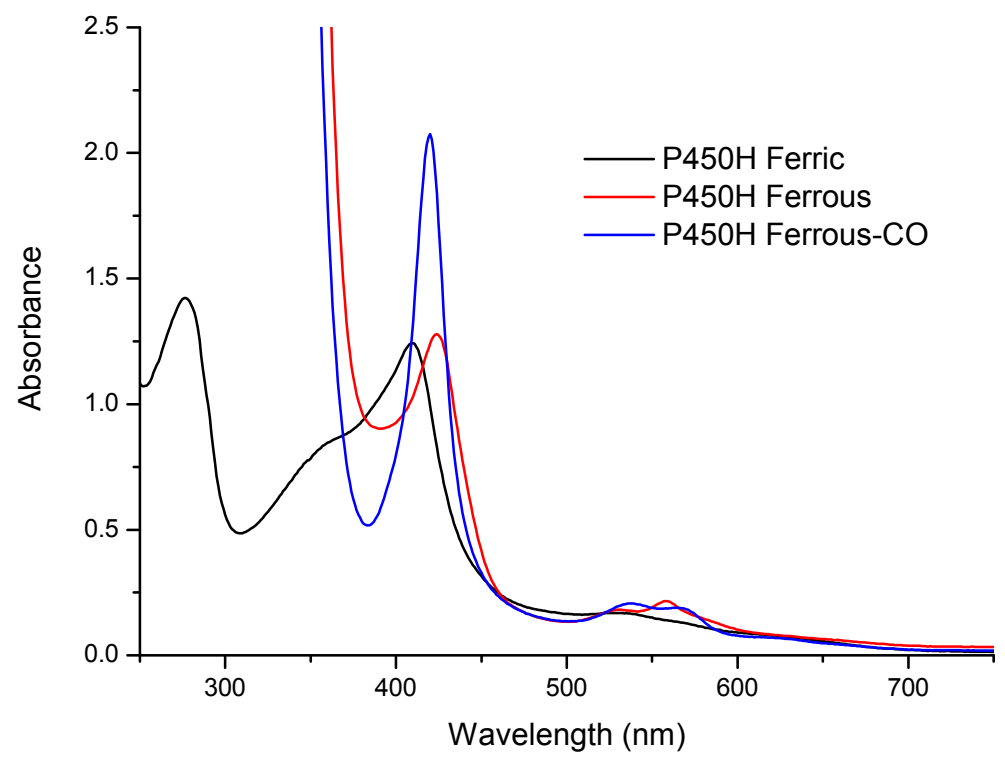

Figure 3.2 UV-visible spectra of $\mathrm{P} 450 \mathrm{H}$ in various oxidation states.

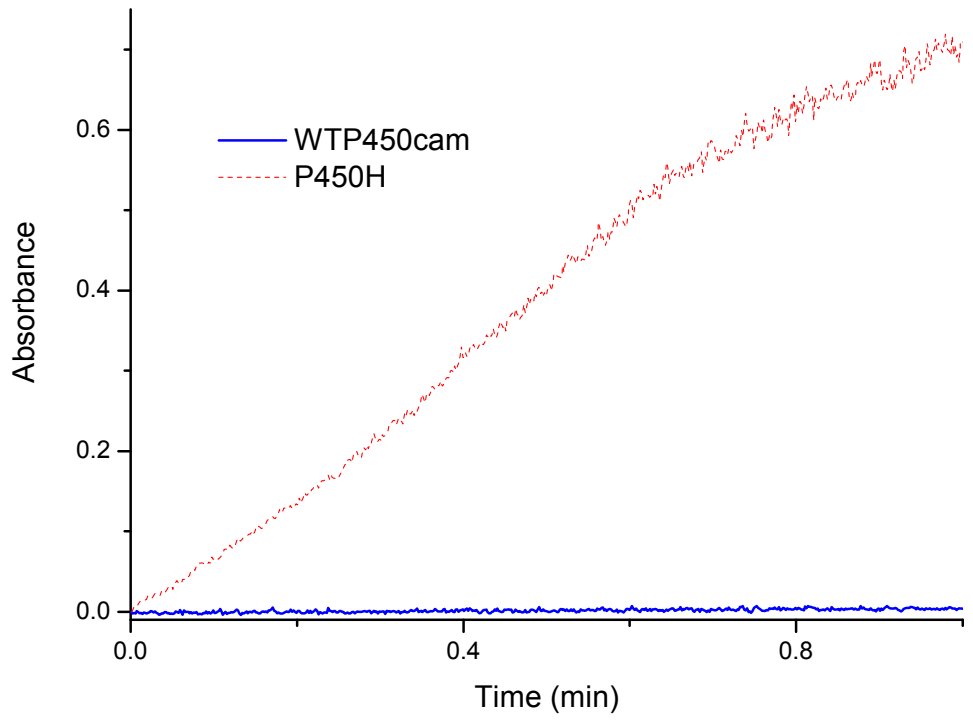

Figure 3.3 Peroxidase activities of wild type $\mathrm{P} 450$ cam and its $\mathrm{P} 450 \mathrm{H}$ mutant determined by monitoring the oxidation of ABTS at $405 \mathrm{~nm}$ in the presence of $\mathrm{H}_{2} \mathrm{O}_{2}(3.3 \mathrm{mM})$, ABTS $(9.1 \mathrm{mM})$, and enzyme $(2 \mu \mathrm{M})$. 
polarity in distal side gained some peroxidase activities, a peroxidase assay using ABTS as the substrate was performed that showed greatly increased activity of $\mathrm{P} 450 \mathrm{H}$ compared with that of wild type protein (Fig. 3.3). These results suggested the modified distal environment of P450cam induced some marked changes both structurally and functionally.

\subsubsection{UV-visible spectroscopic studies of P450L246K}

To examine specifically which one or which ones of the seven substituted residues in the distal pocket caused the dramatic change, single mutation and multiple mutations were carried out. It turned out that the single mutant L246K exhibited quite similar properties with those of $\mathrm{P} 450 \mathrm{H}$. The identity of the mutant proteins was verified by SDS-PAGE. Changing L246, a non-polar residue located on the distal heme of the protein, to lysine, a basic and polar residue resulted in a mutant typical of ferric low spin, six-coordinate heme proteins as demonstrated by the electronic absorption spectra of the mutant (Fig. 3.4). The absorption maxima of the spectra in Figure 3.4 are essentially identical to those of the substrate-free wild-type P450cam and P420cam (Table 3.1), suggesting that the proximal heme iron ligand, Cys357, in wild-type P450cam was retained in the L246K mutant $(34,44)$. However, the electronic absorption spectra of the ferrous and the ferrous-CO complex of the L246K mutant displayed absorption maximum at 423 and $419 \mathrm{~nm}$, rather than at 408 and $450 \mathrm{~nm}$, respectively, expected for 
most heme thiolate proteins. It has been generally believed that these spectral features are characteristics of hemoproteins with proximal histidine ligand (45).

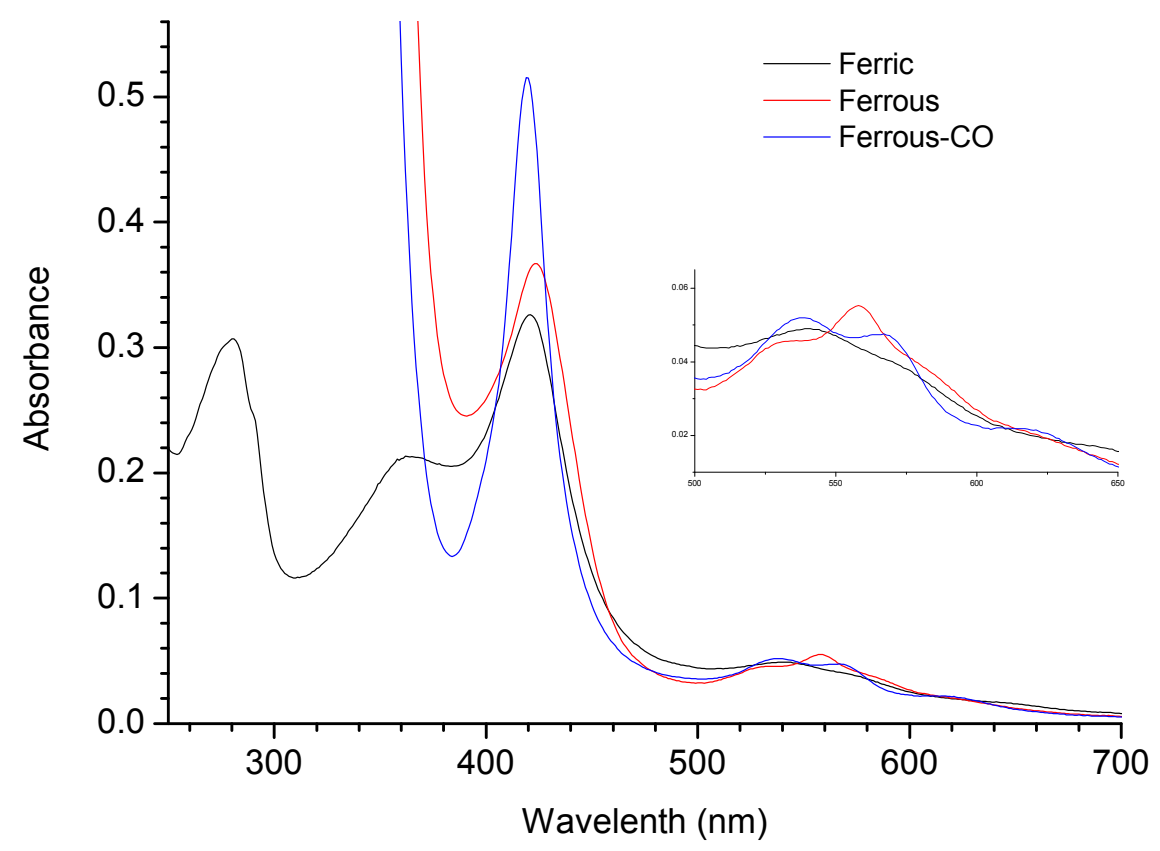

Figure 3.4 UV-vis spectra of P450camL246K in various oxidation states. The inset shows a magnification of the spectra in the $\alpha / \beta$ band region. The enzyme concentration employed was $4 \mu \mathrm{M}$.

However, histidine ligation in this case has been ruled out due to the following two considerations. First, there is no histidine in the distal pocket of P450cam (9). Second, the ferrous and ferrous-CO forms of the P450 L246K-3H mutant $(\mathrm{H} 352 \mathrm{G} / \mathrm{H} 355 \mathrm{G} / \mathrm{H} 361 \mathrm{~A})$ where all three histidines that locate in the vicinity of the proximal helix have been replaced by glycine or alanine, display virtually identical optical absorption features to those of P420 and L246K (Table 3.1). Therefore, it is proposed that the observed absorption maximum at $420 \mathrm{~nm}$ in L246K and L246K-3H mutants as well as in P420 is 
due to milder changes such as protonation of the proximal ligand or lengthening of the Fe-S bond caused by conformational changes induced by mutation or conditions used in the preparation of $\mathrm{P} 420$ rather than more drastic changes such as ligand switching.

Table 3.1 Wavelength and extinction coefficients of absorption maxima for WT P450 cam, $\mathrm{P} 420_{\text {cam }}$, $\mathrm{P} 450_{\text {cam }} \mathrm{L} 246 \mathrm{~K}$ and $\mathrm{P} 450_{\text {cam }} \mathrm{L} 246 \mathrm{~K} 3 \mathrm{H}$

\begin{tabular}{lllll}
\hline Protein & & Soret $(\mathbf{n m})$ & $\boldsymbol{\delta}$-band (nm) & Visible (nm) \\
\hline \multirow{4}{*}{ WT P450cam } & $\mathrm{Fe}^{3+}$ & 417 & 360 & 535,569 \\
& $\mathrm{Fe}^{2+}$ & 408 & & 540 \\
& $\mathrm{Fe}^{2+}-\mathrm{CO}$ & 446 & & 550 \\
\hline \multirow{3}{*}{ P420cam (22) } & $\mathrm{Fe}^{3+}$ & 422 & 367 & $541,566,651$ \\
& $\mathrm{Fe}^{2+}$ & 424 & & 530,558 \\
& $\mathrm{Fe}^{2+}-\mathrm{CO}$ & 420 & & 540,572 \\
\hline \multirow{4}{*}{ P450camL246K } & $\mathrm{Fe}^{3+}$ & 421 & 362 & 540 \\
& $\mathrm{Fe}^{2+}$ & 423 & & 532,558 \\
& $\mathrm{Fe}^{2+}-\mathrm{CO}$ & 419 & & $539,567,620$ \\
\hline \multirow{2}{*}{ P450camL246K } & $\mathrm{Fe}^{3+}$ & 411 & 360 & 535,569 \\
3H & $\mathrm{Fe}^{2+}$ & 423 & & 531,558 \\
& $\mathrm{Fe}^{2+}-\mathrm{CO}$ & 420 & & $540,569,621$ \\
\hline
\end{tabular}

Indeed, thiol-ligated ferrous heme-CO adducts of both synthetic heme model compounds and biomimetic protein models display Sort absorption maximum at $420 \mathrm{~nm}$ $(39,46)$ Alternatively, it has been reported that lengthening of the Fe-S bond by $0.2 \AA$ could induce the optical spectral transition of ferrous-CO complex of heme thiolate proteins from $\sim 450 \mathrm{~nm}$ to $\sim 420 \mathrm{~nm}(34,41)$. Alternatively, protonation of the native proximal thiolate ligand to a neutral thiol could be responsible for the P420-like spectral 
pattern of both L246K and L246K-3H mutants. In fact, neutral thiol-ligated ferrous heme-CO adducts of both synthetic heme model compounds and biomimetic protein models displayed absorption maximum at $420 \mathrm{~nm}(39,46,47)$.

\subsubsection{Resonance Raman spectroscopic study}

The resonance Raman (RR) spectra of heme proteins are characterized in the high frequency region by strong porphyrin in-plane vibrational bands, which are diagnostic of the oxidation-, spin-, and coordination-state of the heme iron (48-50). To further examine the heme environment of L246K and L246K-3H mutants together with wild type P450cam and P420, we obtained resonance Raman spectra in various oxidation states. The RR spectra of all the ferric protein samples displayed a strong oxidation state marker band $v_{4}$ at around $1370 \mathrm{~cm}^{-1}$ that is typical for ferric $\left(\mathrm{Fe}^{3+}\right)$ heme (Fig. $3.5 \mathrm{~A}$ ). The camphor-bound P450cam heme core-size marker bands $v_{3}$ and $v_{2}$ appeared at $1486 \mathrm{~cm}^{-1}$ and $1568 \mathrm{~cm}^{-1}$, respectively, indicating a five-coordinate (5C), high-spin (HS) heme iron (51-53). While for all other samples, the spectra displayed the $v_{3}$ and $v_{2}$ bands at around $1500 \mathrm{~cm}^{-1}$ and $1580 \mathrm{~cm}^{-1}$, suggesting a six-coordinate (6C), low-spin (LS) heme iron component. Overall the camphor-free, camphor-bound P450cam and P420 RR spectra agree well with previous RR studies $(54,55)$. The spectra of both ferric L246K and L246K-3H closely resemble that of ferric P420, with mainly 6C-LS heme iron in each case (Fig. 3.5 A). 

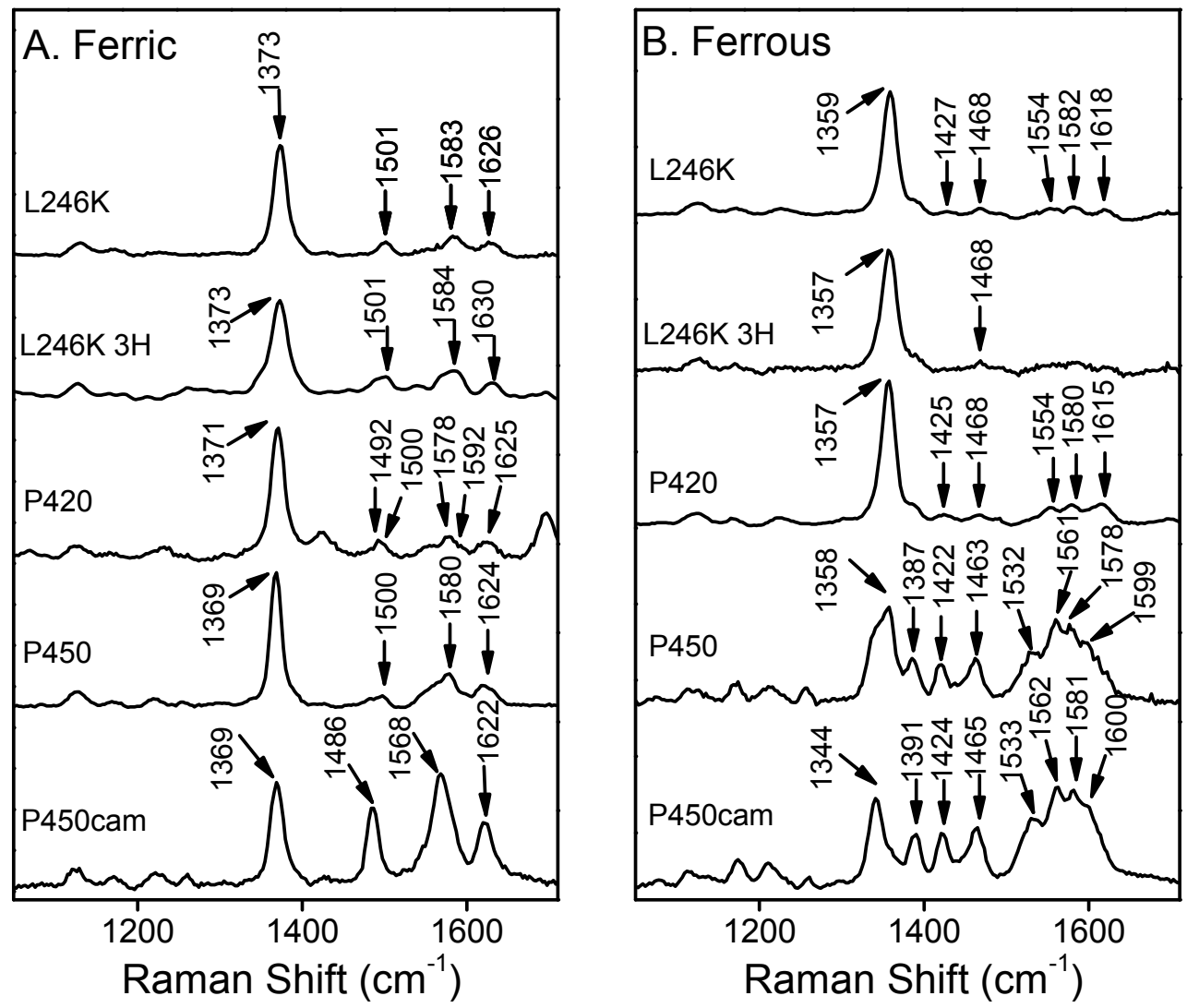

Figure 3.5 Resonace Raman spectra of (A) ferric and (B) ferrous wild-type P450 (in the presence and absence of $1 \mathrm{mM}$ camphor), P420, P450L246K mutant and P450L246K 3H mutant. The excitation wavelength $\left(\lambda_{\text {ex }}\right)$ was $413.1 \mathrm{~nm}$.

The $v_{4}$ band in the RR spectrum for $5 \mathrm{C}-\mathrm{Fe}^{2+}$ heme complexes is a useful diagnostic marker of the identity of the proximal ligand. RR studies of a wide variety of heme proteins have shown that $5 \mathrm{C}-\mathrm{Fe}^{2+}$ heme complexes with histidine (imidazole) as proximal ligand produce a $v_{4}$ band in the $1350-1360 \mathrm{~cm}^{-1}$ region whereas those with a cysteine (thiolate) proximal ligand display a $v_{4}$ band in the $1340-1348 \mathrm{~cm}^{-1}$ region $(56,57)$. The RR spectrum of ferrous P450cam shows a $v_{4}$ band at at $1344 \mathrm{~cm}^{-1}$ that is consistent with thiolate proximal ligation (Fig. 3.5B). The $v_{3}$ and $v_{2}$ band at $1465 \mathrm{~cm}^{-1}$ and $1562 \mathrm{~cm}^{-1}$ 
confirm that the ferrous $\mathrm{P} 450$ cam complex is 5C-HS. The RR spectrum of ferrous $\mathrm{P} 420$ also indicates a mainly 5C-HS complex but in contrast to P450cam the $v_{4}$ band occurs at $1357 \mathrm{~cm}^{-1}$, which is consistent with proximal histidine ligation (Fig. 3.5B). The RR spectra of ferrous L246K and L246K-3H mutants are almost identical to that of P420, thus ruling out the possibility that the high value for the position of the $v_{4}$ band is associated with proximal histidine ligation in this case.

An alternative possibility is that for ferrous P420, L246K and L246K 3H mutants the proximal ligand is the protonated cysteine (neutral thiol) form (58). Assignment of the

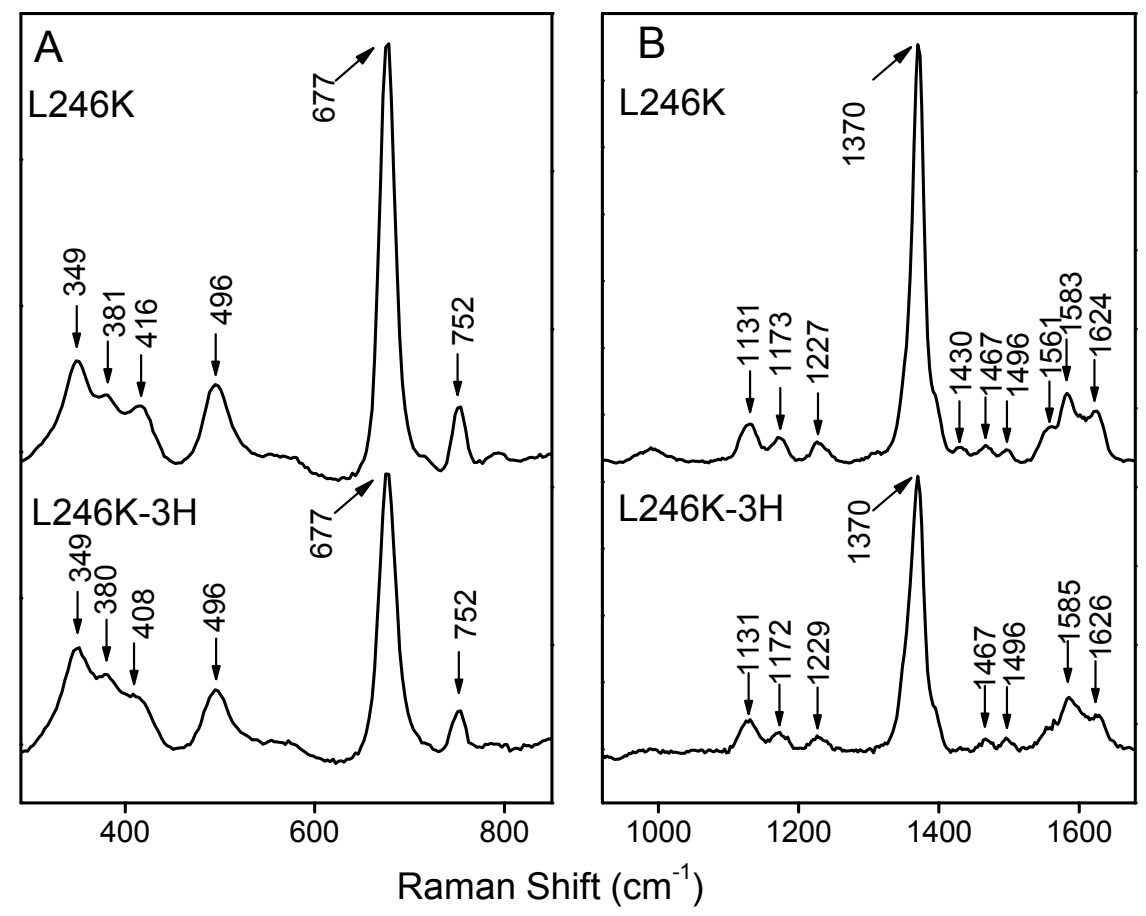

Figure 3.6 Resonace Raman spectra of $\mathrm{Fe}^{\mathrm{II}}-\mathrm{CO}$ complexes of $\mathrm{P} 450$ camL246K and L246K-3H mutants in both low frequency region (A) and high frequency region (B). 
$\mathrm{Fe}-\mathrm{CO}$ and $\mathrm{C}-\mathrm{O}$ stretching modes of the $\mathrm{Fe}{ }^{\mathrm{II}}-\mathrm{CO}$ complexes is useful because they are influenced by the nature of proximal ligand and the polarity of the distal pocket environment $(59,60)$. The RR spectra of the $\mathrm{Fe}^{\mathrm{II}}-\mathrm{CO}$ complex of $\mathrm{L} 246 \mathrm{~K}$ mutant in the high-frequency region displayed a characteristic six-coordinate low-spin pattern, with peaks at around $1,370 \mathrm{~cm}^{-1}(v 4), 1,496 \mathrm{~cm}^{-1}$ (v3), and 1,583 $\mathrm{cm}^{-1}$ (v2) (Fig. $3.6 \mathrm{~B}$ ). The L246K-3H showed almost identical six-coordinate features, indicating that it shared very similar heme environment with L246K. The $v_{\mathrm{Fe}-\mathrm{CO}}$ bands for both of them were both of them were seen at $496 \mathrm{~cm}^{-1}$. Confirmation of these assignments was provided by the observed shifts in the presence of ${ }^{13} \mathrm{C}^{18} \mathrm{O}$ (Fig. 3.7 A). In addition, $v_{\mathrm{C}-\mathrm{O}}$ for L246K was at approximately $1960 \mathrm{~cm}^{-1}$ in the ${ }^{12} \mathrm{C}^{16} \mathrm{O}-{ }^{13} \mathrm{C}^{18} \mathrm{O}$ difference spectrum (Fig. $3.7 \mathrm{~B}$ ). Relating the frequencies of $v_{\mathrm{Fe}-\mathrm{CO}}$ and $v_{\mathrm{C}-\mathrm{O}}$ can provide valuable information about the proximal 


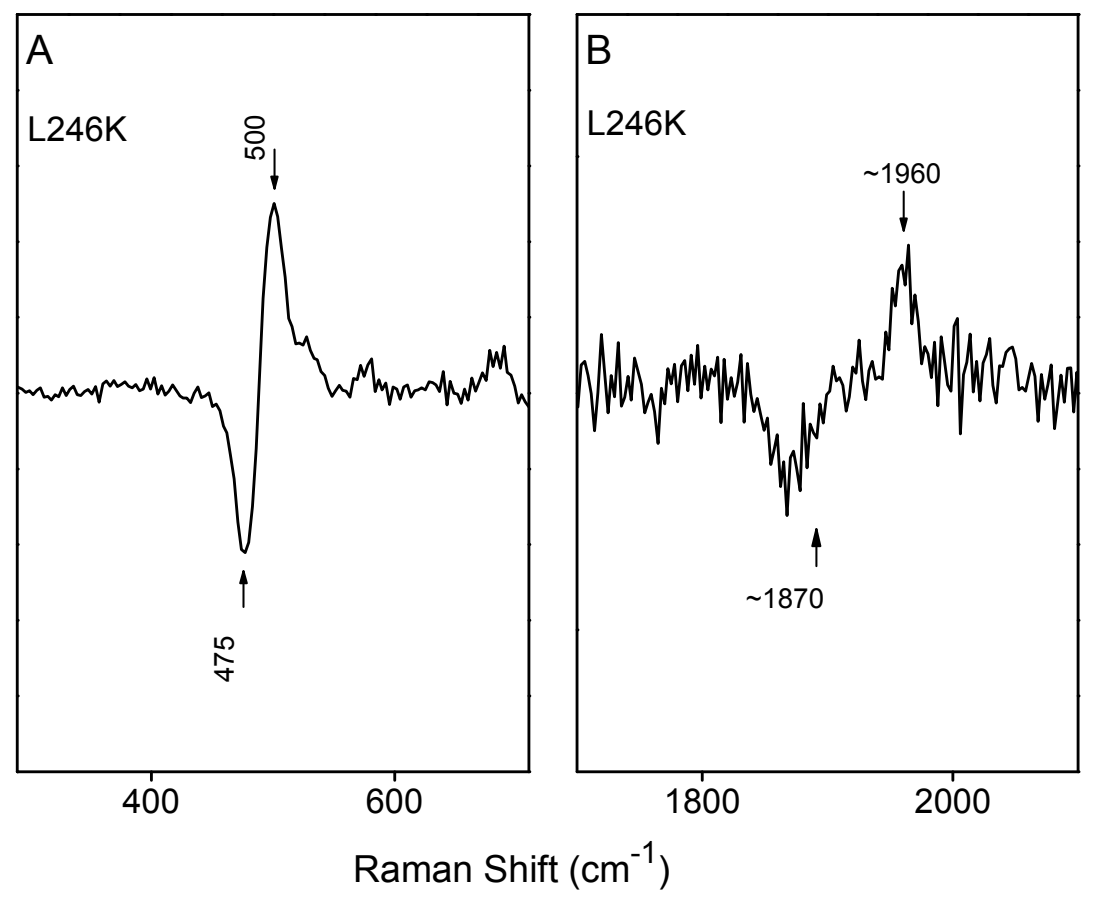

Figure 3.7 $\mathrm{Fe}^{2+}-\mathrm{CO}-\mathrm{Fe}^{2+}-{ }^{13} \mathrm{C}^{18} \mathrm{O}$ difference spectra $\mathrm{P} 450$ camL246K in both low frequency region (A) and high frequency region (B).

ligand identity and polarity of the distal environment of hemoprtoeins (61-63). Plots of $v_{\mathrm{Fe}-\mathrm{CO}}$ versus $v_{\mathrm{C}-\mathrm{O}}$ for a wide variety of heme-CO complexes are shown in Figure 3.8. The observed 500 and $1960 \mathrm{~cm}^{-1}$ paring frequency for the CO complex of L246K mutant placed it in the region of histidine proximal ligand and a hydrophobic distal environment compared with myoglobin and peroxidases. Since the proximal histidine ligand has been ruled out in the $\mathrm{L} 246 \mathrm{~K}$ mutant, the $\mathrm{RR}$ results of the $\mathrm{CO}$ complex could be indicative of a protonated thiolate proximal ligation or an elongated iron-thiolate ligand. 


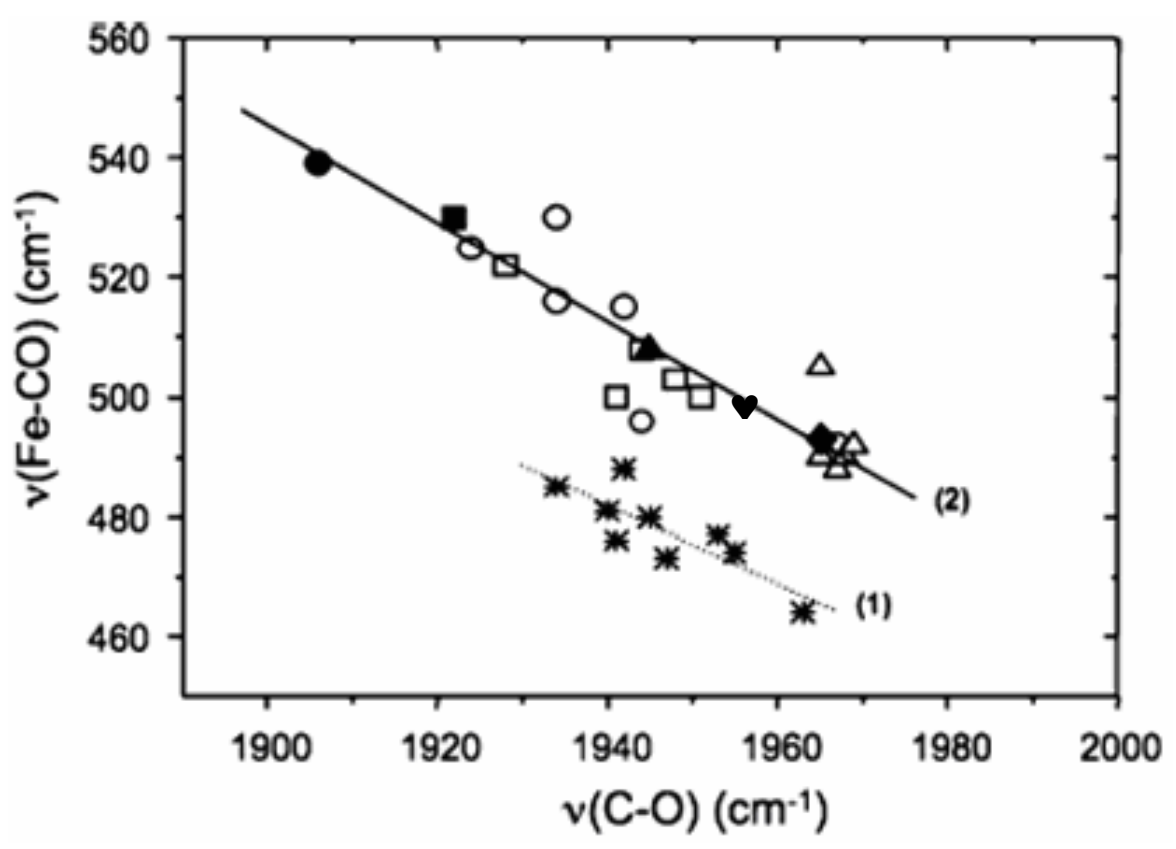

Figure 3.8 Correlation of $\mathrm{Fe}-\mathrm{CO}$ and $\mathrm{C}-\mathrm{O}$ stretching frequencies for different heme proteins and porphyrin derivatives. Lines 1 and 2 are for complexes in which the proximal ligand is thiolate and histidine, respectively. The open circles, open squares, and open triangles correspond to data are obtained for mutant and $\mathrm{pH}$-variant forms of horseradish peroxidase ( $\mathrm{O})$, cytochrome $\mathrm{c}$ peroxidase ( $\square$ ), and myoglobin $(\Delta)$, respectively. The corresponding filled symbols represent data are obtained from the wild-type proteins. The filled heart corresponds to P450L246K data measured in this work. This figure is adapted from (61-63).

\subsubsection{Catalytic activity of P450L246K}

While it still remains challenging to distinguish between a protonated neutral thiol and an elongated thiolate ligand in heme proteins that display abnormal spectroscopic properties, it is generally agreed that the deprotonation of the proximal cysteine to cysteinate that provides the necessary "push" effect throughout the reaction cycle is indispensable for the catalytic activity of all known heme thiolate proteins. $(1,7-9,47,64)$ Therefore, an activity assay should provide a convenient measure for the identification of 


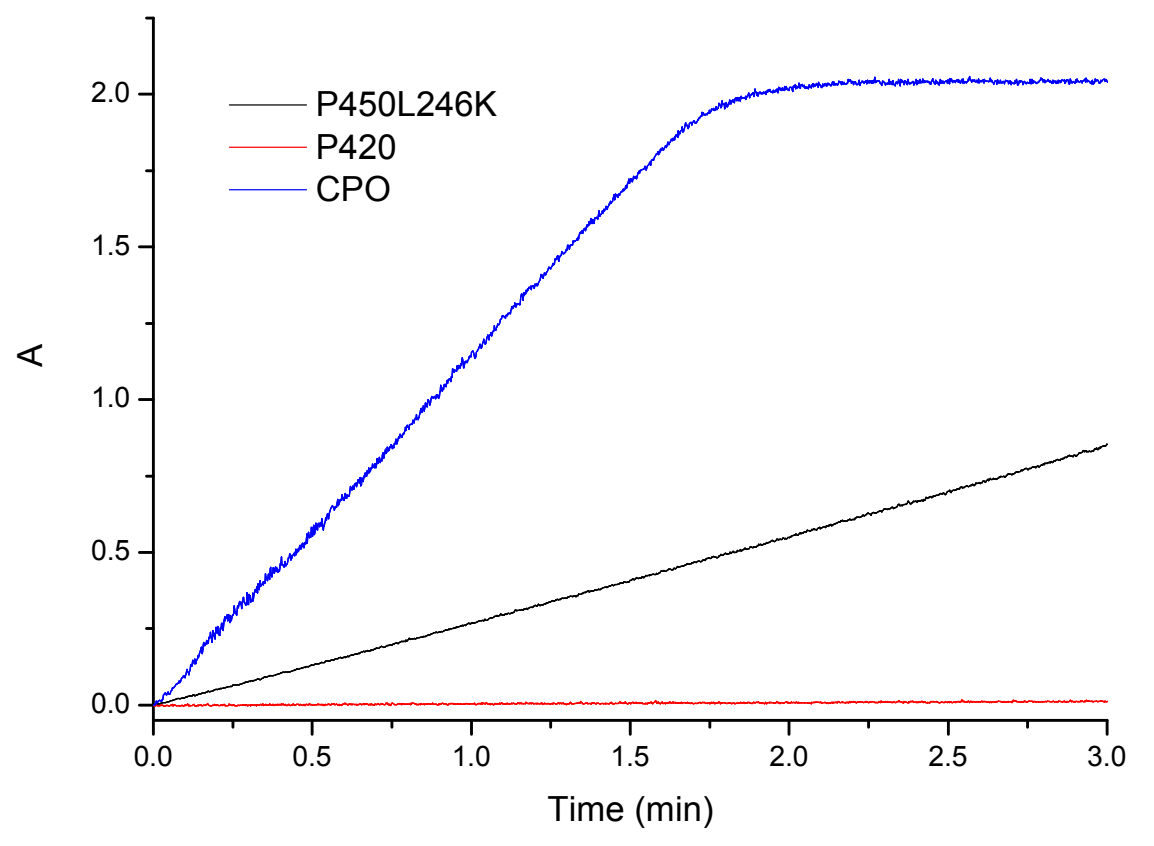

Figure 3.9 Peroxidase activity of P450camL246K mutant, P420 and CPO determined by monitoring the oxidation of ABTS at $405 \mathrm{~nm}$ in the presence of $\mathrm{H}_{2} \mathrm{O}_{2}(3.3 \mathrm{mM})$, ABTS $(9.1 \mathrm{mM})$, and enzyme $(2 \mu \mathrm{M})$.

the nature of the proximal ligand in these proteins. Although both P420 and L246K display essentially identical UV-vis spectral patterns, the proximal ligand to the heme iron may not necessarily be the same. The fact that P420 is devoid of any catalytic activity suggests a protonated cysteine as the proximal heme ligand in this inactivated form of P450. However, the ABTS assay of L246K has shown that the mutant is able to act as an efficient peroxygenase (Fig. 3.9), with comparable peroxidase activity with CPO, via the peroxide shunt pathway. That implies that a deprotonated cysteinate ligates weakly to the heme iron of the mutant with a lengthened Fe-S bond. The ability of L246K to oxidize ABTS, a standard peroxidase substrate, in the presence of $\mathrm{H}_{2} \mathrm{O}_{2}$ alone 
strongly supports the importance of polarity of heme distal environment in regulating the chemistry of heme proteins as predicted by structural comparison of CPO and P450cam.

In conclusion, our study shows that it is possible to engineer P450cam into a self-sufficient biocatalyst by changing the polarity of the distal heme environment of the protein. Our results provide strong evidence that the inactivated form of P450 (P420) uses a protonated cysteine as its proximal heme iron ligand while L246K keeps the parent protein's thiolate ligand but with a longer Fe-S bond due to conformational changes induced by the mutation. It is therefore concluded that proteins with identical spectral signatures do not necessarily share the same heme environment.

\subsection{REFERENCES}

1. Ortiz de Montellano, P. R., (Ed.) (2005) Cytochrome P450: Structure, Mechanism, and Biochemistry, 3rd ed., Kluwer Academic/Plenum Publishers, New York.

2. Sono, M., Roach, M. P., Coulter, E. D., and Dawson, J. H. (1996) Heme-Containing Oxygenases, Chem. Rev. 96, 2841-2888.

3. Rabe, K., Gandubert, V., Spengler, M., Erkelenz, M., and Niemeyer, C. (2008) Engineering and assaying of cytochrome P450 biocatalysts, Anal. Bioanal. Chem. 392, 1059-1073.

4. Bernhardt, R. (2006) Cytochromes P450 as versatile biocatalysts, J. Biotechnol. 124, 128-145.

5. Poulos, T. L., Finzel, B. C., and Howard, A. J. (1987) High-Resolution Crystal-Structure of Cytochrome-P450cam, Journal of Molecular Biology 195, 687-700. 
6. Sigel, A., Sigel, H., and Sigel, R. K.O. (2007) The ubiquitous roles of cytochrome P450 proteins in Metal Ions in Life Sciences (Sigel, A., Sigel, H., and Sigel, R. K.O. , Ed.), Wiley, Chichester.

7. Dawson, J. H., Holm, R. H., Trudell, J. R., Barth, G., Linder, R. E., Bunnenberg, E., Djerassi, C., and Tang, S. C. (1976) Magnetic circular dichroism studies. 43. Oxidized cytochrome P-450. Magnetic circular dichroism evidence for thiolate ligation in the substrate-bound form. Implications for the catalytic mechanism, $J$. Am. Chem. Soc. 98, 3707-3709.

8. Tarr, G. E., Black, S. D., Fujita, V. S., and Coon, M. J. (1983) Complete amino acid sequence and predicted membrane topology of phenobarbital-induced cytochrome P-450 (isozyme 2) from rabbit liver microsomes, Proc. Natl. Acad. Sci. U. S. A. 80, 6552-6556.

9. Poulos, T. L., Finzel, B. C., Gunsalus, I. C., Wagner, G. C., and Kraut, J. (1985) The 2.6-A crystal structure of Pseudomonas putida cytochrome P-450, J. Biol. Chem. 260, 16122-16130.

10. Sono, M. R., M. P.; Coulter, E. D.; and Dawson, J. H. (1996) Heme-Containing Oxygenases, Chemical Reviews 96, 2841-2847.

11. Behan, R. K., Stone, K. L., Hoffart, L. M., Krebs, C., and Green, M. T. (2006) Evidence for protonated ferryls in compound II of cytochromes P450, J. Am. Chem. Soc. 128, 11471-11474.

12. Dawson, J. H., and Sono, M. (1987) Cytochrome P-450 and Chloroperoxidase: Thiolate-Ligated Heme Enzymes. Spectroscopic Determination of Their Active Site Structures and Mechanistic Implications of Thiolate Ligation, Chemical Reviews 87, 1255-1276.

13. Dawson, J. H. (1988) Probing structure-function relations in hemecontaining oxygenases and preoxidases, Science 240, 433-439.

14. Katagiri, M., Ganguli, B. N., and Gunsalus, I. C. (1968) A Soluble Cytochrome P-450 Functional in Methylene Hydroxylation, J. Biol. Chem. 243, 3543-3546.

15. Omura, T., Sato, R. (1964) The Carbon Monoxide-binding Pigment of Liver Microsomes: I. EVIDENCE FOR ITS HEMOPROTEIN NATURE, J. Biol. Chem. 239, 2370-2378. 
16. Yu, C.-A., Gunsalus, I .C. (1974) Cytochrome P-450cam: II. Interconversion with P-420, J. Biol. Chem. 249, 102-106.

17. Hui Bon Hoa, G., Di Primo, C., Dondaine, I., Sligar, S. G., Gunsalus, I. C., Douzou, P. (1989) Conformational Changes of Cytochromes P-450cam, and P-450lin Induced by High Pressuret, Biochemistry 28, 651-656.

18. FISHER, M. T., SCARLATA, S. F., SLIGAR, S. G. (1985) High-Pressure investigations of Cytochrome P-450 Spin and Substrate Binding Equilibria, Arch. Biochem. Biophys. 240, 456-463.

19. Imai, Y., Sato, R. (1967) Conversion of P-450 to P-420 by Neutral Salts and some other Reagents, Eur. J. Biochem. 1, 419-426.

20. O'Keefe, D. H., Ebel, R. E., Peterson, J. A., Maxwell, J. C., Caughey, W. S. (1978) An infrared spectroscopic study of carbon monoxide bonding to ferrous cytochrome P-450, Biochemistry 17, 5845-5852.

21. Sundaramoorthy, M., Terner, J., and Poulos, T. L. (1995) The crystal structure of chloroperoxidase: A heme peroxidase-cytochrome P450 functional hybrid, Structure 3, 1367-1377.

22. Martinis, S. A., Blanke, S. R., Hager, L. P., Sligar, S. G., Hoa, G. H. B., Rux, J. J., and Dawson, J. H. (1996) Probing the heme iron coordination structure of pressure-induced cytochrome P420cam, Biochemistry 35, 14530-14536.

23. Hui Bon Hoa, G., Di Primo, C., Dondaine, I., Sligar, S. G., Gunsalus, I. C., and Douzou, P. (1989) Conformational changes of cytochromes P-450cam and P-450lin induced by high pressure, Biochemistry 28, 651-656.

24. Hui Bon Hoa, G., Di Primo, C., Geze, M., Douzou, P., Kornblatt, J. A., and Sligar, S. G. (1990) The formation of cytochrome P-450 from cytochrome P-420 is promoted by spermine, Biochemistry 29, 6810-6815.

25. Ichikawa, Y., and Yamano, T. (1967) Reconversion of detergent- and sulfhydryl reagent-produced P-420 to P-450 by polyols and glutathione, Biochim. Biophys. Acta 131, 490-497.

26. Stern, J. O., Peisach, J. J. (1974) A Model Compound Study of the CO-Adduct of Cytochrome P-450, J. Biol. Chem. 249, 7495-7498. 
27. Sono, M., Stuehr, D. J., Ikeda-Saito, M., Dawson, J. H. (1995) Identification of Nitric Oxide Synthase as a Thiolate-ligated Heme Protein Using Magnetic Circular Dichroism Spectroscopy: COMPARISON WITH CYTOCHROME P-450-CAM AND CHLOROPEROXIDASE, J. Biol. Chem. 270, 19943-19948.

28. Dunford, A. J., McLean, K. J., Sabri, M., Seward, H. E., Heyes, D. J., Scrutton, N. S., Munro, A. W. . (2007) Rapid P450 Heme Iron Reduction by Laser Photoexcitation of Mycobacterium tuberculosis CYP121 and CYP51B1: ANALYSIS OF CO COMPLEXATION REACTIONS AND REVERSIBILITY OF THE P450/P420 EQUILIBRIUM, J. Biol. Chem. 282, 24816-24824.

29. Hui Bon Hoa, G., McLean, M. A., Sligar, S. G. (2002) High pressure, a tool for exploring heme protein active sites, Biochim. Biophys. Acta 1595, 297-308.

30. White, K. A., Marletta, M. A. (1992) Nitric oxide synthase is a cytochrome P-450 type hemoprotein, Biochemistry 31, 6627-6631.

31. Sabat, J., Stuehr, D. J., Yeh, S. R., and Rousseau, D. L. (2009) Characterization of the Proximal Ligand in the P420 Form of Inducible Nitric Oxide Synthase, Journal of the American Chemical Society 131, 12186-12192.

32. Blanke, S. R., Martinis, S. A., Sligar, S. G., Hager, L. P., Rux, J. J., Dawson, J. H. (1996) Probing the Heme Iron Coordination Structure of Alkaline Chloroperoxidase, Biochemistry 35, 14537-14543.

33. Hollengerg, P. F., Hager, L. P. (1973) The P450-nature of the carbon monoxide complex of ferrous chloroperoxidase, J. Biol. Chem. 248, 2630-2633.

34. Martinis, S. A., Blanke, S. R., Hager, L. P., Sligar, S. G., Hui Bon Hoa, G., Rux, J. J., and Dawson, J. H. (1996) Probing the Heme Iron Coordination Structure of Pressure-Induced Cytochrome P420cam, Biochemistry 35, 14530-14536.

35. Jiang, Y., Trnka, M. J., Medzihradszky, K. F., Ouellet, H., Wang, Y., and Ortiz de Montellano, P. R. (2009) Covalent heme attachment to the protein in human heme oxygenase-1 with selenocysteine replacing the His 25 proximal iron ligand, $J$. Inorg. Biochem. 103, 316-325. 
36. Vetter, S., Terentis, A., Osborne, R., Dawson, J., and Goodin, D. (2009) Replacement of the axial histidine heme ligand with cysteine in nitrophorin 1: spectroscopic and crystallographic characterization, J. Biol. Inorg. Chem. 14, 179-191.

37. Dawson, J. H., Trudell, J. R., Linder, R. E., Barth, G., Bunnenberg, E., and Djerassi, C. (1978) Magnetic circular dichroism of purified forms of rabbit liver cytochromes P-450 and P-420, Biochemistry 17, 33-42.

38. Wells, A. V., Li, P., Champion, P. M., Martinis, S. A., and Sligar, S. G. (1992) Resonance Raman investigations of Escherichia coli-expressed Pseudomonas putida cytochrome P450 and P420, Biochemistry 31, 4384-4393.

39. Perera, R., Sono, M., Sigman, J. A., Pfister, T. D., Lu, Y., and Dawson, J. H. (2003) Neutral thiol as a proximal ligand to ferrous heme iron: Implications for heme proteins that lose cysteine thiolate ligation on reduction, Proc. Natl. Acad. Sci. U. S. A. 100, 3641-3646.

40. Hanson, L. K., Eaton, W. A., Sligar, S. G., Gunsalus, I. C., Gouterman, M., and Connell, C. R. (1976) Origin of the anomalous Soret spectra of carboxycytochrome P-450, J. Am. Chem. Soc. 98, 2672-2674.

41. Jung, C. (1985) Quantum chemical explanation of the "hyper" spectrum of the carbon monoxide complex of cytochrome P-450, Chem. Phys. Lett. 113, 589-596.

42. Liu, Y., Moenne-Loccoz, P., Hildebrand, D. P., Wilks, A., Loehr, T. M., Mauk, A. G., and Ortiz de Montellano, P. R. (1999) Replacement of the Proximal Histidine Iron Ligand by a Cysteine or Tyrosine Converts Heme Oxygenase to an Oxidase, Biochemistry 38, 3733-3743.

43. Wagner, G. C., Perez, M., Toscano, W. A. Jr., and Gunsalus, I. C. (1981) Apoprotein Formation and Heme Reconstitution of Cytochrome P-450cam, $J$. Biol. Chem. 256, 6262-6265.

44. Dawson, J. H., Andersson, L. A., and Sono, M. (1982) Spectroscopic investigations of ferric cytochrome P-450-CAM ligand complexes. Identification of the ligand trans to cysteinate in the native enzyme, J. Biol. Chem. 257, 3606-3617. 
45. Omura, T. (2005) Heme-thiolate proteins, Biochem. Biophys. Res. Commun. 338, 404-409.

46. Collman, J. P., Sorrell, T. N., Dawson, J. H., Trudell, J. R., Bunnenberg, E., and Djerassi, C. (1976) Magnetic circular dichroism of ferrous carbonyl adducts of cytochromes P-450 and P-420 and their synthetic models: further evidence for mercaptide as the fifth ligand to iron, Proc. Natl. Acad. Sci. U. S. A. 73, 6-10.

47. Sabat, J., Stuehr, D. J., Yeh, S.-R., and Rousseau, D. L. (2009) Characterization of the Proximal Ligand in the P420 Form of Inducible Nitric Oxide Synthase, J. Am. Chem. Soc. 131, 12186-12192.

48. Andersson, L. A., Mylrajan, M., Sullivan, E. P., and Strauss, S. H. (1989) Modeling low-pH hemoproteins, J. Biol. Chem. 264, 19099-19102.

49. Hu, S. Z., Morris, I. K., Singh, J. P., Smith, K. M., and Spiro, T. G. (1993) Complete Assignment of Cytochrome-C Resonance Raman-Spectra Via Enzymatic Reconstitution with Isotopically Labeled Hemes, J. Am. Chem. Soc. $115,12446-12458$.

50. Hu, S. Z., Smith, K. M. and Spiro, T. G. . (1996) Assignment of protoheme Resonance Raman spectrum by heme labeling in myoglobin, J. Am. Chem. Soc. $118,12638-12646$.

51. Andersson, L. A., Mylrajan, M., Sullivan, E. P., Jr., and Strauss, S. H. (1989) Modeling low-pH hemoproteins, J Biol Chem 264, 19099-19102.

52. Lou, B. S., Snyder, J. K., Marshall, P., Wang, J. S., Wu, G., Kulmacz, R. J., Tsai, A. L., and Wang, J. (2000) Resonance Raman studies indicate a unique heme active site in prostaglandin H synthase, Biochemistry 39, 12424-12434.

53. Spiro, T. G., Stong, J. D., and Stein, P. (1979) Porphyrin Core Expansion and Doming in Heme-Proteins - New Evidence from Resonance Raman-Spectra of 6-Coordinate High-Spin Iron(Iii) Hemes, Journal of the American Chemical Society 101, 2648-2655.

54. Wells, A. V., Li, P., Champion, P. M., Martinis, S. A., and Sligar, S. G. (1992) Resonance Raman investigations of Escherichia coli-expressed Pseudomonas putida cytochrome P450 and P420, Biochemistry 31, 4384-4393. 
55. Champion, P. M., Gunsalus, I. C., and Wagner, G. C. (1978) Resonance Raman Investigations of Cytochrome P450cam from Pseudomonas-Putida, Journal of the American Chemical Society 100, 3743-3751.

56. Anzenbacher, P., Evangelista-Kirkup, R., Schenkman, J., and Spiro, T. G. (1989) Influence of thiolate ligation on the heme electronic structure in microsomal cytochrome P-450 and model compounds: resonance Raman spectroscopic evidence, Inorg. Chem. FIELD Full Journal Title:Inorganic Chemistry 28, 4491-4495.

57. Wang, J., Caughey, W. S., and Rousseau, D. L. (1996) Resonance Raman Scattering: a Probe of Heme Protein-bound Nitric Oxide, in Methods in Nitric Oxide Research (Feelisch, M., and Stamler, J., Eds.), pp 427-454, J. Wiley, Chichester ; New York.

58. Perera, R., Sono, M., Sigman, J. A., Pfister, T. D., Lu, Y., and Dawson, J. H. (2003) Neutral thiol as a proximal ligand to ferrous heme iron: implications for heme proteins that lose cysteine thiolate ligation on reduction, Proc Natl Acad Sci U S A 100, 3641-3646.

59. Spiro, T. G., Kozlowski, P. (2001) Acc. Chem. Res. 34, 137-144.

60. Vogel, K. M., Kozlowski, P.M., Zgierski, M.Z., Spiro T.G. (2000) Inorg. Chim. Acta. 297, 11-17.

61. Li, T., Quillin, M.L., Phillips, G.N., Olson, J.S. (1994) Structural Determinants of the Stretching Frequency of $\mathrm{CO}$ Bound to Myoglobin, Biochemistry 33, 1433-1446.

62. Feis, A., Rodriguez-Lopez, J.N., Thorneley, R.N.F., Smulevich, G. (1998) The Distal Cavity Structure of Carbonyl Horseradish Peroxidase As Probed by the Resonance Raman Spectra of His 42 Leu and Arg 38 Leu Mutants, Biochemistry $37,13575-13581$.

63. Terentis, A. C., Thomas, S. R., Takikawa, O., Littlejohn, T. K., Truscott, R. J. W., Armstrong, R. S., Yeh, S. R., and Stocker, R. (2002) The heme environment of recombinant human indoleamine 2,3-dioxygenase - Structural properties and substrate-ligand interactions, Journal of Biological Chemistry 277, 15788-15794. 
64. Schlichting, I., Berendzen, J., Chu, K., Stock, A. M., Maves, S. A., Benson, D. E., Sweet, R. M., Ringe, D., Petsko, G. A., and Sligar, S. G. (2000) The Catalytic Pathway of Cytochrome P450cam at Atomic Resolution, Science 287, 1615-1622. 


\section{CHAPTER IV. SPECTROSCOPIC STUDY OF A NOVEL HEME IRON LIGAND SET IN ENGINEERED CYTOCHROME P450CAM}

\subsection{SUMMARY}

Substitution of glycine-248, the distal residue closest to the heme iron of cytochrome P450cam, with a histidine leads to the formation of a mutant protein with a novel Cys-Fe-His ligand set. This is one of the rarest cases of His ligation in heme-thiolate proteins, and represents the first example observed for P450cam. The G248H mutant shows very unique electronic absorption properties. The ferric form of G248H displays a characteristic Soret band at $425 \mathrm{~nm}$, while its ferrous-CO complex shows the distinctive absorption maximum at approximately $450 \mathrm{~nm}$ indicative of the intact Cys thiolate ligation. Surprisingly, this mutant does not bind any substrates or exogenous ligands and is inert toward $\mathrm{pH}$ changes, suggesting the replacement of the distal water molecule with the introduced His residue as the endogenous ligand. Resonance Raman and circular dichroism spectroscopy results also support this model. The spectrum of the ferric form of $\mathrm{G} 248 \mathrm{H}$ displayed a six-coordinate low spin pattern. The spectrum of ferrous mutant showed a mixture of six-coordinate low spin and five-coordinate high spin, indicating that the distal His-Fe bond is weakened in the ferrous form. The results have also provided evidence about the nature of proximal 
ligation of the P420 species, in which the nearby histidine is not a possible alternative for the heme iron ligand.

\subsection{INTRODUCTION}

Cytochromes P450, a superfamily of heme $b$-containing monooxygenases distributed in all domains of life, are involved in a wide range of physiologically and biotechnologically significant transformations, including steroid synthesis, drug metabolism, xenobiotic detoxification, and molecular signaling (1-4). The P450 enzymes typically activate dioxygen and insert one oxygen atom into a wide variety of organic substrates. The catalytic versatility makes these enzymes of great interest to the biochemical and potentially industrial applications. The most distinctive structural feature of cytochromes P450 is the coordination of a deprotonated cysteine to the heme iron as the proximal ligand, while many other hemoproteins possess a histidine at the proximal side. The strong electron-releasing character of the thiolate ligand has been reported to serve as the "push" effect to facilitate the heterolytic O-O bond cleavage to generate the activated intermediate Cpd I (iron-(IV)-oxo species) (5). With a few exceptions, the diverse reactions catalyzed by $\mathrm{P} 450 \mathrm{~s}$ all requires an external source of reducing equivalents like $\mathrm{NAD}(\mathrm{P}) \mathrm{H}$ and auxiliary proteins to transfer electrons to $\mathrm{P} 450$. The requirement of expensive cofactors and the complication caused by additional proteins is

the limitation to the application of P450s as oxygenases. Some P450s are capable of 
accepting hydrogen peroxide or organic peroxy compound as an oxidant to catalyze oxygen insertion through the so-called "peroxide-shunt" pathway in the absence of electron transport proteins and the $\mathrm{NAD}(\mathrm{P}) \mathrm{H}$ cofactor (6-11). However, P450s are typically not efficient in their utilization of hydrogen peroxide, the natural substrate for peroxidases, as the source of oxygen atom in the monooxygenation reactions.

Cytochrome P450cam from Pseudomonus putida is one of the most well studied members in the superfamily which catalyzes the region- and stereospecific hydroxylation of its natural substrate $d$-camphor to 5-exo-hydroxy-camphor (12). A great deal of effort has been made to suitably engineer this enzyme by directed evolution and active site modification for the oxygenation of significant unnatural substrates. It is still quite important to understand the roles of the active-site components in the reactivity, stability, specificity and substrate recognition of this enzyme after its discovery for over 50 years.

To explore the possibility of converting the cytochrome P450cam into an efficient self-sufficient heme peroxidase, we attempted to modify the heme distal side elements which play crucial mechanistic roles and regulate the enzyme functions. In most heme peroxidases, the distal histidine is highly conserved in the active site and has been considered to serve as a general acid-base catalyst necessary for the formation of Cpd I by donating a proton to the $\mathrm{Fe}^{3+}-\mathrm{OOH}$ moiety to promote the heterolytic cleavage of the $\mathrm{O}-\mathrm{O}$ bond in the peroxidase reaction cycles (13-16). It has been proposed that a functional nitrogen (such as the nitrogen of His42 in HRP and His52 in $\mathrm{CcP}$ ), located at a 
suitable distance from the heme iron, could behave as an acid-base catalyst in hemoproteins $(13,17)$.

Therefore, in order to probe the roles of the nonpolar amino acids in the distal helix and to make an efficient peroxygenase, we tried to substitute a distal residue with a hydrophilic histidine. Given the proximity of the Gly248 residue to the heme group in P450cam, it is a rational to choose this residue as the target for our purpose (Fig. 4.1). The Gly248 residue, serving as a hydrogen bond partner with the side chain of Thr252 and resulting in an I-helix kink $(18,19)$, is conserved as a small residue (Gly or Ala) in many P450s. In the previous study of P450 variants with the Gly or Ala substituted by a carboxylate reside (Glu), the carboxylate group was reported to coordinate with the heme iron, resulting in a six-coordinate ferric resting state in both P450cam and P450BM3 (20-22). Thus, Gly248 in P450cam has been chosen as a good candidate to explore the consequences of functional alteration of the heme distal pocket on P450 structure and catalysis. Surprisingly, the results of the G248H variant did not show any catalytic activities at all because of the novel ligate set generated with the mutation. It is demonstrated to contain a similar heme coordination with the P450BM3 A264H mutant that was reported to possess a unique Cys-Fe-His heme ligand set (23). However, the G248H mutant of P450cam was rather different from its parallel model in the P450BM3 variant, especially the heme coordination in its ferrous and ferrous- $\mathrm{CO}$ complexes. It is shown for the first time that thiolate ligation remains intact without being displaced by 

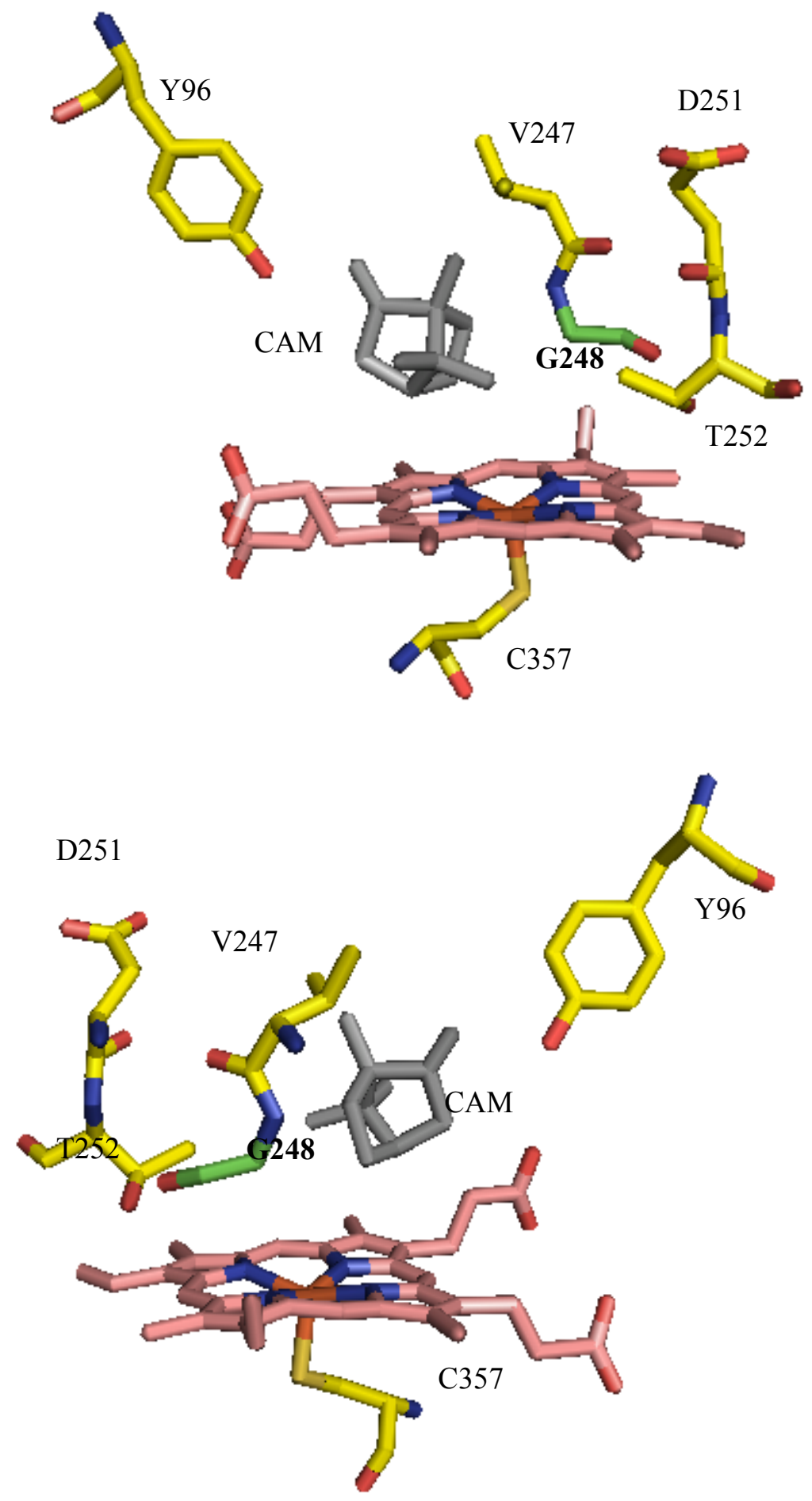

Figure 4.1 The active site structure of camphor-bound cytochrome P450cam showing the position of some important residues including G248. The figures were generated from the crystal data (PDB code 2CPP) using PYMOL (18). 
another coordination group or deprotonated in various oxidation states either in modified heme-thiolate proteins or in other hemoproteins with engineered proximal thiolate ligation.

In this study various spectroscopic methods, such as electronic absorption, resonance Raman, and circular dichroism have been applied to investigate the heme coordination and ligation state of the heme active site in the G248H mutant. It has always been difficult to identify a ligand trans to thiolate in the absence of structural detail, but here we successfully presented sufficient spectroscopic results that identify axial ligand trans to the cysteinate in this distinctive mutant of P450cam.

\subsection{EXPERIMENTAL PROCEDURES}

\subsubsection{Site-directed Mutagenesis}

Site-directed mutagenesis was performed by using this T7-based expression vector, pET-30a $(+)$. The $\mathrm{G} 248 \mathrm{H}$ mutant of $\mathrm{P} 450_{\text {cam }}$ was prepared with the 5 '-sense

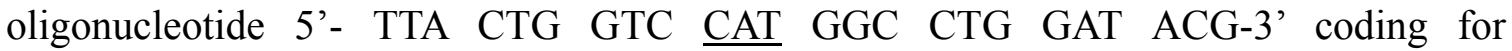
LLV HGLDT. The reconstituted plasmids of both wild type and G248H mutant were transformed into E. coli strain NovaBlue and verified by DNA sequencing.

\subsubsection{Protein Expression and Purification}

The wild type P450cam and mutant G248H were expressed in Escherichia coli strain Rosetta II (DE3). Cells were grown on terrific broth supplemented with $100 \mathrm{mg} / \mathrm{L}$ 
of kanamycin at $37{ }^{\circ} \mathrm{C}$ and induced with $1 \mathrm{mM}$ IPTG and $1 \mathrm{mM}$ camphor at $\mathrm{OD}_{600}$ around 0.8 , followed by further growth at $30{ }^{\circ} \mathrm{C}$ for another $18 \mathrm{~h}$ prior to harvesting cells. As for the mutant, the addition of $\delta$-aminolevulinic acid to the growth media is necessitated to facilitate holoenzyme production.

Cells were then lysed at $4{ }^{\circ} \mathrm{C}$ in $50 \mathrm{mM}$ Tris- $\mathrm{HCl}$ buffer at $\mathrm{pH} 7.4$ containing $50 \mathrm{mM}$ $\mathrm{KCl}, 0.5 \mathrm{mM}$ DTT, $1 \mathrm{mM}$ camphor (buffer A), 1 mM EDTA, $200 \mu \mathrm{M}$ PMSF, $40 \mathrm{U} / \mathrm{ml}$ Dnase I, $3 \mathrm{U} / \mathrm{ml}$ Rnase A, and lysozyme. Following sonication and centrifugation, the lysed cells were concentrated by Amicon cell with 30,000 Da cut-off ultrafiltration membrane. The protein solution was applied on a fast flow DEAE Sepharose column (50ml) equilibrated with buffer A. After initial elution, the column was developed with a linear $0-0.5 \mathrm{M} \mathrm{KCl}$ gradient in the same buffer. The red fractions containing the $\sim 45 \mathrm{kDa}$ proteins were pooled for further purification by size exclusion chromatography. Sephadex G-75 matrix $(500 \mathrm{ml})$ was used for the gel filtration and the buffer applied was $100 \mathrm{mM}$ potassium phosphate buffer containing $1 \mathrm{mM}$ camphor at $\mathrm{pH}$ 7.4. Substrate-free WTP450cam and its mutant were prepared in the same way except for no camphor was added during the expression and purification procedures. Unless otherwise specified, all the steps in the protein purification were performed at $4{ }^{\circ} \mathrm{C}$.

\subsubsection{Spectroscopic characterization}

Optical characterization of the wild type $\mathrm{P} 450_{\text {cam }}$ and its mutant was performed at room temperature on a Cary 300 spectrophotometer (Varian). The ferrous proteins were prepared by the addition of excess amount of sodium dithionite and $\mathrm{CO}$ adducts were 
obtained by $\mathrm{CO}$ gas bubbling. The buffer used for the absorption measurements was 100 $\mathrm{mM}$ potassium phosphate buffer with $1 \mathrm{mM}$ camphor at $\mathrm{pH}$ 7.4.

CD spectra were measured on a JASCO J-815 spectrometer at room temperature. The secondary-structure region $(200-260 \mathrm{~nm})$ of the spectra was recorded in a quartz cuvette of $1 \mathrm{~mm}$ path length using about $2 \mu \mathrm{M}$ enzyme. As for the tertiary-structure region (260-650 nm), they were recorded using about $20 \mu \mathrm{M}$ enzyme in a quartz cuvette of $1 \mathrm{~cm}$ path length with and without camphor.

For the resonance Raman data collection, the wild-type P450 and G248H mutant samples (about $30 \mu \mathrm{M}$ ) were prepared in a septum-sealed, cylindrical quartz cell. Samples were reduced to the ferrous form by first flushing the sample with argon and then injecting a molar excess of buffered sodium dithionite solution. The rotating sample cell was irradiated with $4 \mathrm{~mW}$ of $413.1 \mathrm{~nm}$ laser light using a mixed krypton/argon ion laser (Spectra Physics, Beamlok 2060). The spectral acquisition time was 5 min. The scattered light was collected at right angles to the incident beam and focused onto the entrance slit $(125 \mu \mathrm{m})$ of a $0.8 \mathrm{~m}$ spectrograph, where it was dispersed by a 600 groove/mm grating and detected by a liquid- $\mathrm{N}_{2}$-cooled $\mathrm{CCD}$ camera (Horiba-JY). Spectral calibration was performed against the lines of mercury. UV-vis spectra of each sample were collected before and after each RR measurement to ensure the sample integrity. 


\subsubsection{Redox potential measurements}

The electrochemical measurements of the $\mathrm{G} 248 \mathrm{H}$ mutant were performed with a CHI 660 electrochemical analyzer (CH Instruments) at room temperature by using a conventional three electrode cell, with a glassy carbon (GC) electrode as the working electrode, platinum wire as counter electrode and $\mathrm{Ag} / \mathrm{AgCl}$ electrode as the reference electrode. The carbon electrode has been modified with single-walled carbon nanotubes (SWCNT). The SWCNT modified electrode is electrochemically activated by cyclical scanning between -1.0 and $+1.5 \mathrm{~V}$ at a scan rate of $1 \mathrm{~V} / \mathrm{sec}$ for 2 minutes to improve the sensitivity of the electrode. The redox potentials of P450cam G248H mutant was obtained and normalized with the reference to NHE.

\subsection{RESULTS}

\subsubsection{Protein expression and purification}

The G248H mutant of P450cam was expressed as a holoprotein with the facilitation of heme precursor. The $\mathrm{Rz}$ values of pure wild-type P450cam and G248H mutant were both over 1.4. The protein concentration was determined using the Soret extinction coefficient determined by the pyridine hemochrome method following the standard protocol $(24,25)$. The extinction coefficients for camphor-bound and camphor-free forms of the mutant

were found to be 113 and $125 \mathrm{mM}^{-1} \mathrm{~cm}^{-1}$, respectively, comparing with 102 and 115 $\mathrm{mM}^{-1} \mathrm{~cm}^{-1}$ of the wild type protein. 


\subsubsection{UV-vis spectroscopy}

A

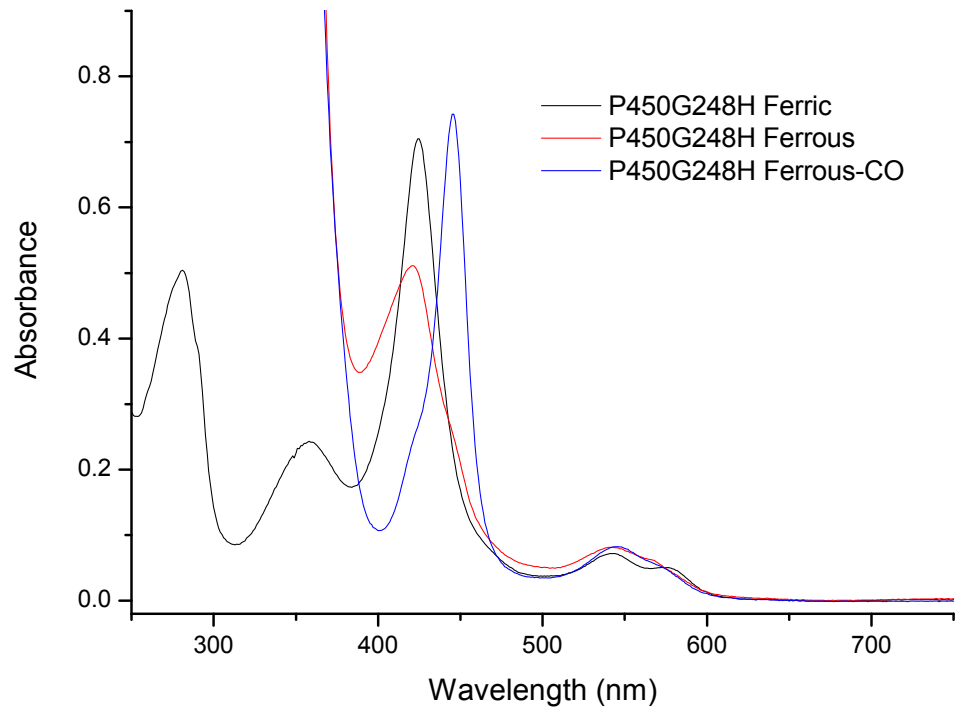

B

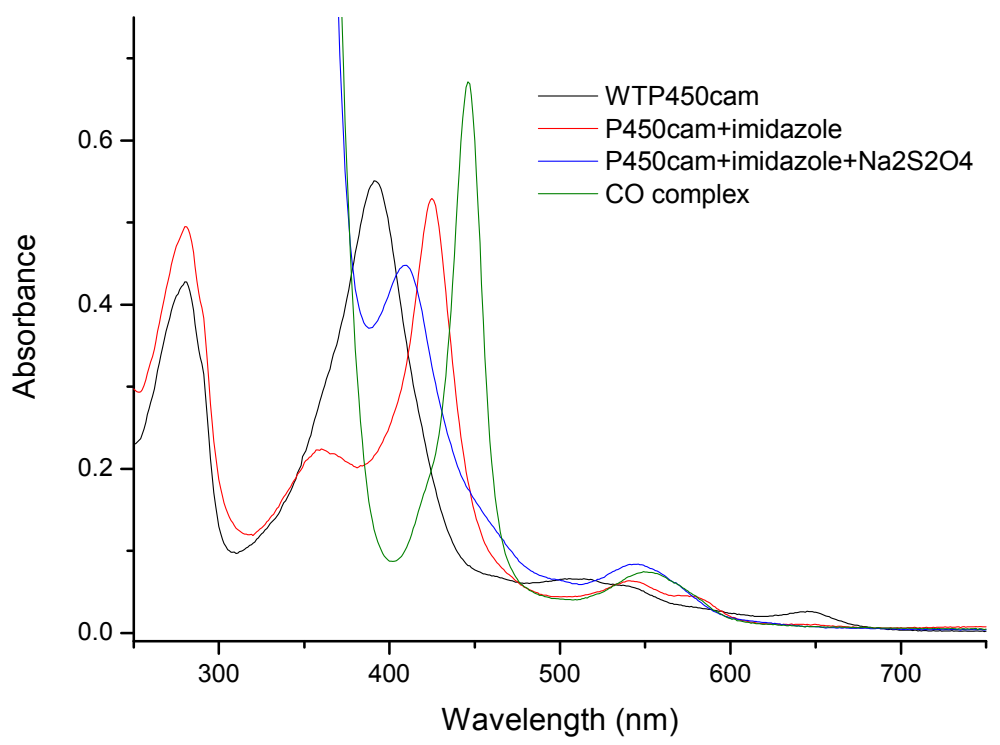

Figure 4.2 UV-vis spectra of ferric, ferrous and ferrous-CO complexes of P450camG248H: (A) camphor-free; (B) camphor-bound in 100mM phosphate buffer ( $\mathrm{pH} 7.4)$ at room temperature. 
The UV-vis spectra of wild type P450cam and G248H mutant were recorded in all ferric, ferrous and ferrous carbon monoxide bound forms (Table 4.1). In contrast to the substrate-free wild type protein in its oxidized state that has the Soret maximum at 417 $\mathrm{nm}$, the G248H mutant exhibited a Soret band at $425 \mathrm{~nm}$, red shifted by $8 \mathrm{~nm}$ (Fig. 4.2) Notably, the ferric form of the mutant displayed an almost identical spectral pattern with that of the wild type protein complexed with imidazole (26) and that of the P450BM3 A264H mutant (23), indicating that the substitution of the Gly248 with a histidine residue similar to those proteins in these proteins. Furthermore, the UV-vis spectrum of ferric $\mathrm{P} 450$ camG248H did not show any change upon any ligand or substrate binding, and is independent of $\mathrm{pH}$ change as well. However, the ferrous form of the mutant was quite different from the wild type and its imidazole complex. Instead of showing the Soret band at around $408 \mathrm{~nm}$ upon reduction, the G248H mutant had its Soret band maximum at $421 \mathrm{~nm}$. The ferrous-CO complex of the G248H mutant retained the characteristic Soret band at around 450nm like the wild type P450cam, which suggested that despite the introduction of a sterically larger group and also a stronger ligand, the thiolate ligation to the heme iron in the proximal side was preserved.

Another distinct UV-vis spectral difference between the mutant and wild type lies in the spectra of their camphor-bound forms. In wild type P450cam, the Soret band shifts from $417 \mathrm{~nm}$ to $392 \mathrm{~nm}$ upon substrate binding, indicating the conversion of low-spin to high-spin Fe (III). But the camphor binding was perturbed in the mutant since there was 
no detectable change in its camphor-bound form. Thus, in the presence of camphor, the G248H mutant remained predominantly in the six-coordinate low-spin Fe (III) state.

Table 4.1 Wavelength and extinction coefficients of absorption maxima for wild type and G248H mutant of cytochrome P450cam

\begin{tabular}{lllll}
\hline Protein & Oxidation state & Soret $(\mathrm{nm})$ & $\delta$-band (nm) & Visible (nm) \\
\hline WTP450 & $\mathrm{Fe}^{3+}$ & $418(\varepsilon=115)$ & 360 & 535,569 \\
(camphor-free) & $\mathrm{Fe}^{2+}$ & 408 & 540 \\
& $\mathrm{Fe}^{2+}-\mathrm{CO}$ & 446 & 550 \\
\hline WTP450+ & $\mathrm{Fe}^{3+}$ & $425(\varepsilon=98)$ & 360 & 541,578 \\
Imidazole (26) & $\mathrm{Fe}^{2+}$ & 409 & 543 \\
\hline G248H & $\mathrm{Fe}^{2+}-\mathrm{CO}$ & 446 & 549 \\
(camphor-free) & $\mathrm{Fe}^{3+}$ & $425(\varepsilon=123)$ & 359 & 542,573 \\
& $\mathrm{Fe}^{2+}$ & 421 & 541,568 \\
\hline G248H & $\mathrm{Fe}^{2+}-\mathrm{CO}$ & 446 & 545 \\
\hline (camphor-bound) & $\mathrm{Fe}^{3+}$ & $424(\varepsilon=122)$ & 359 & 542,574 \\
& $\mathrm{Fe}^{2+}-\mathrm{CO}$ & 446 & 542,569 \\
\hline
\end{tabular}




\subsubsection{Resonance Raman Spectroscopy}

To further examine its heme environment and the iron spin and ligation state, we obtained RR spectra in various oxidation and coordination states of wild type and G248H mutant in the absence and presence of substrate camphor. The RR spectrum of ferric G248H with no camphor bound was very similar with to that of the wild type, displaying a strong oxidation state marker band $v_{4}$ at around $1370 \mathrm{~cm}^{-1}$ that is typical of ferric $\left(\mathrm{Fe}^{3+}\right)$ heme (Fig. $4.3 \mathrm{~A}$ ). The heme core-size marker bands $v_{3}$ and $v_{2}$ appeared at proximately 1500 and $1580 \mathrm{~cm}^{-1}$, respectively for both camphor-free samples, indicating a six-coordinate (6C), low-spin (LS) heme iron (27-29). However for the camphor-bound proteins, the G248H mutant was markedly different from the wild type. The wild type displayed the $v_{3}$ and $v_{2}$ bands at $1486 \mathrm{~cm}^{-1}$ and $1568 \mathrm{~cm}^{-1}$, respectively, typical of highspin, five-coordinate (5C), heme iron component. On the contrary, the camphor-bound G248H was demonstrated to be a six-coordinate low-spin complex, as indicated by the appearance of the $v_{3}$ and $v_{2}$ bands at 1500 and $1586 \mathrm{~cm}^{-1}$, almost identical to the camphorfree samples. This could be the result of an unusual axial ligation of the heme iron in the G248H mutant. 
A: Ferric

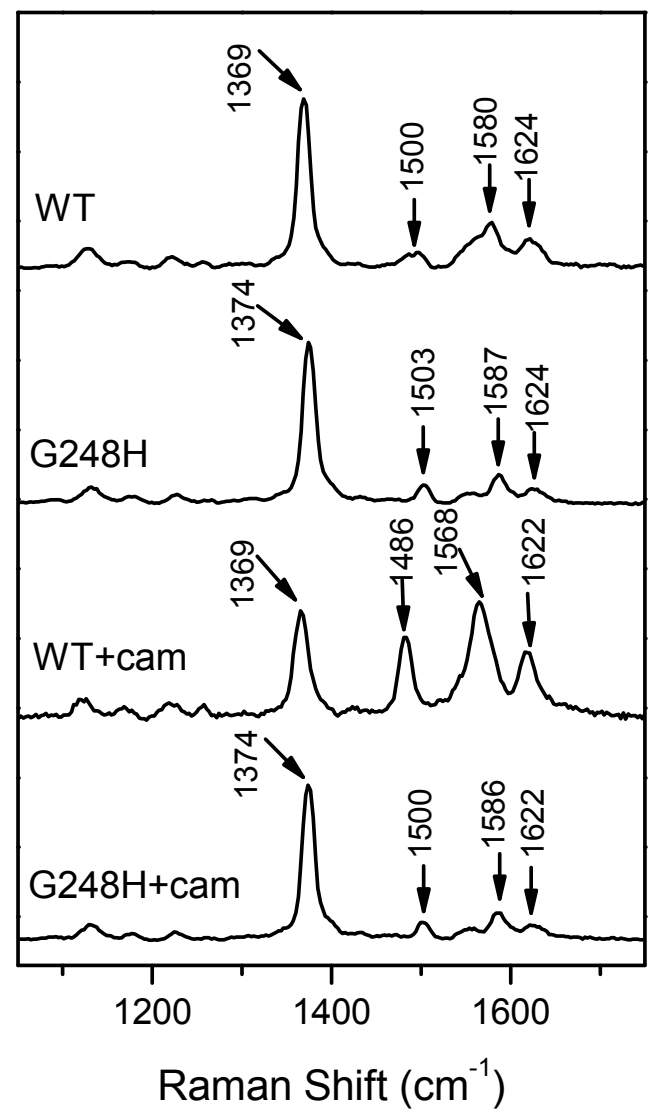

B: Ferrous

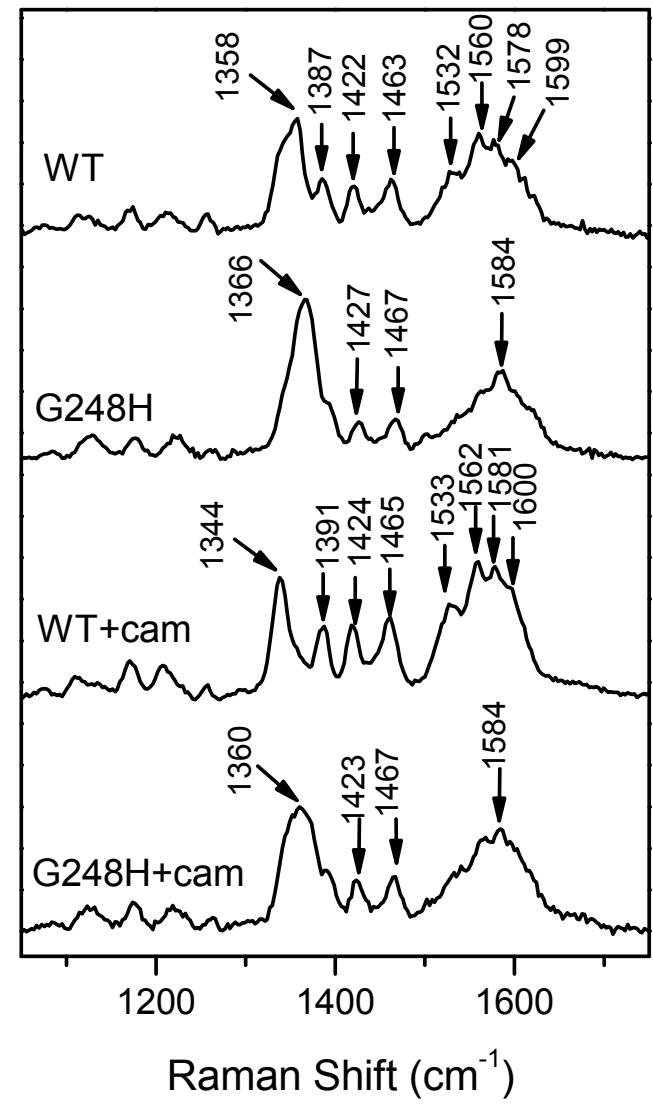

Figure 4.3 Resonance Raman spectra of (A) ferric and (B) ferrous wild-type P450 and P450G248H mutant with and without camphor binding. The excitation wavelength $\left(\lambda_{\text {ex }}\right)$ was $413.1 \mathrm{~nm}$. The spectra of P450 and G248H samples in the presence and absence of $1 \mathrm{mM}$ camphor are shown.

In the ferrous form, the mutant also showed rather different spectral pattern from that of the wild type in the absence and presence of camphor. The RR spectrum of the camphor-free ferrous $\mathrm{G} 248 \mathrm{H}$ had a significantly broadened $v_{4}$ band at $1366 \mathrm{~cm}^{-1}$ that implied a six-coordinate complex and rather different from the wild type (Fig. 4.3 B). 

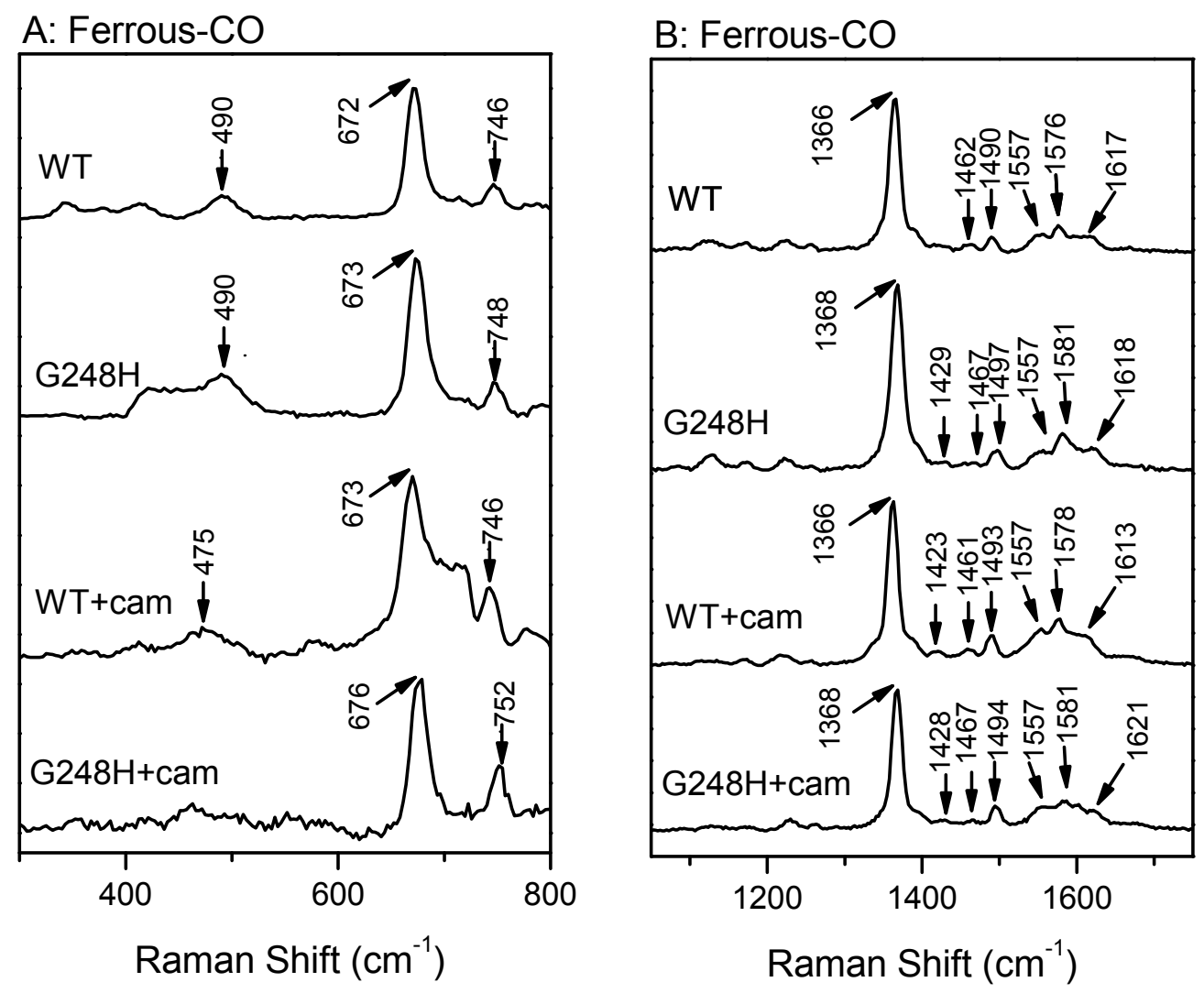

Figure 4.4 Resonance Raman spectra of ferrous-CO complexes of wild-type P450 and P450G248H mutant with and without camphor binding in both low frequency (A) and high frequency (B). The excitation wavelength $\left(\lambda_{\text {ex }}\right)$ was $413.1 \mathrm{~nm}$. The spectra of P450 and G248H samples in the presence and absence of $1 \mathrm{mM}$ camphor are shown.

Typically the $v_{4}$ band appears slightly higher $\left(\sim 1370 \mathrm{~cm}^{-1}\right)$ for six-coordinate ferrous complexes (e.g., CO and NO complexes), which suggested that in the case of G248H the sixth ligand was bonded rather weakly to the heme iron. Consistent with this, a very weak shoulder on the low frequency edge of the $v_{4}$ band could be seen in the G248H RR spectrum that was the result of a small population of the five-coordinate thiolate complex. Compared with the camphor free protein, the camphor-bound mutant demonstrated a 
similar spectral pattern except for the $v_{4}$ band which shifted from $1366 \mathrm{~cm}^{-1}$ to $1360 \mathrm{~cm}^{-1}$, possibly due to the interference of camphor in the His binding to the heme iron in some way.

Assignment of the $\mathrm{Fe}-\mathrm{CO}$ and $\mathrm{C}-\mathrm{O}$ stretching modes of the $\mathrm{Fe}^{\mathrm{II}}-\mathrm{CO}$ complexes is useful because they are influenced by the nature of proximal ligand $(30,31)$. The RR spectra of the $\mathrm{Fe}^{\mathrm{II}}-\mathrm{CO}$ complexes of the wild type and $\mathrm{G} 248 \mathrm{H}$ mutant in the high-frequency region displayed a characteristic six-coordinate low-spin pattern, with peaks at about $1368 \mathrm{~cm}^{-1}(v 4), 1495 \mathrm{~cm}^{-1}(v 3)$, and $1580 \mathrm{~cm}^{-1}(v 2)$, respectively (Fig. 4.4 B). The $v_{\mathrm{Fe}-\mathrm{CO}}$ bands showed some difference between the camphor-free and camphor-bound proteins. Especially for G248H with camphor, the $\mathrm{CO}$ stretch at around $500 \mathrm{~cm}^{-1}$ was missing which was possibly due to the preventive interference of the His ligand in the distal site, suggesting CO could only partially bind to the ferrous heme iron and there was still some portion of histidine ligated to the iron.

\subsubsection{Circular dichroism spectroscopy}

To investigate the effect of the mutation on the conformational change of the protein, multi-wavelength $\mathrm{CD}$ studies of the wild type and G248H mutant of P450cam were carried out. The far-UV CD spectra $(190-260 \mathrm{~nm})$ for both substrate-free and substrate-bound forms of $\mathrm{G} 248 \mathrm{H}$ mutant heme domain exhibited no obvious change compared with that of the wild type, with minima at 219 and $209 \mathrm{~nm}$ respectively, indicating the secondary structure was not significantly altered by the substitution of 
G248 with a histidine. Neither did the addition of camphor have any effect on the secondary structure of the protein in the predominantly $\alpha$-helical heme domain.

However, in the near-UV-visible CD region (300-650 $\mathrm{nm})$, the G248H mutant showed rather different spectra in its resting ferric form from that of the wild type. Furthemore, the substrate free and substrate bound forms of the mutant were markedly distinct from each other. In the absence of camphor, the Soret and $\delta$ bands in the heme CD spectrum of wild type P450cam appeared at 410 and $353 \mathrm{~nm}$ in negative ellipticity (Fig. 4.5A). The Soret band shifted to $388 \mathrm{~nm}$ upon substrate binding, with the $\delta$ band disappearing (Fig. 4.5 B). Thus, the blue shift of the Soret band of P450cam mimicked the optical transition in the UV-vis spectrum to some extent. However, the near-UV-visible CD spectrum of the substrate-free G248H heme domain has its Soret band at $423 \mathrm{~nm}$ and the addition of substrate camphor triggered dramatic change in the Soret band, resulting in split peaks at 405 and $436 \mathrm{~nm}$, respectively. This feature of the substrate-bound G248H mutant was more significantly different than that of the optical absorption spectra. 


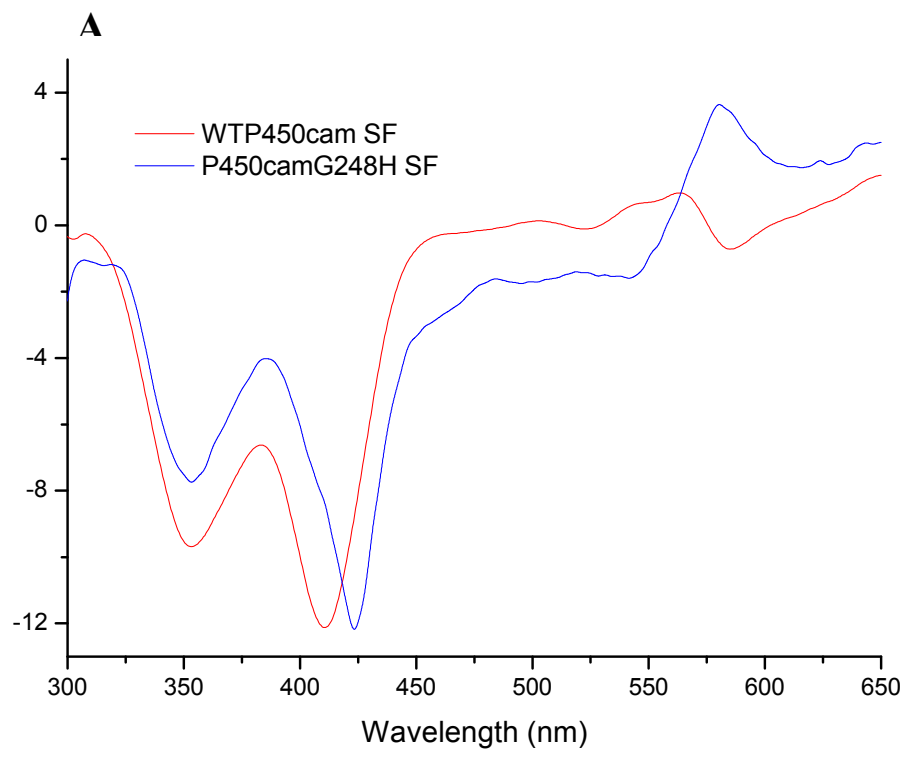

B

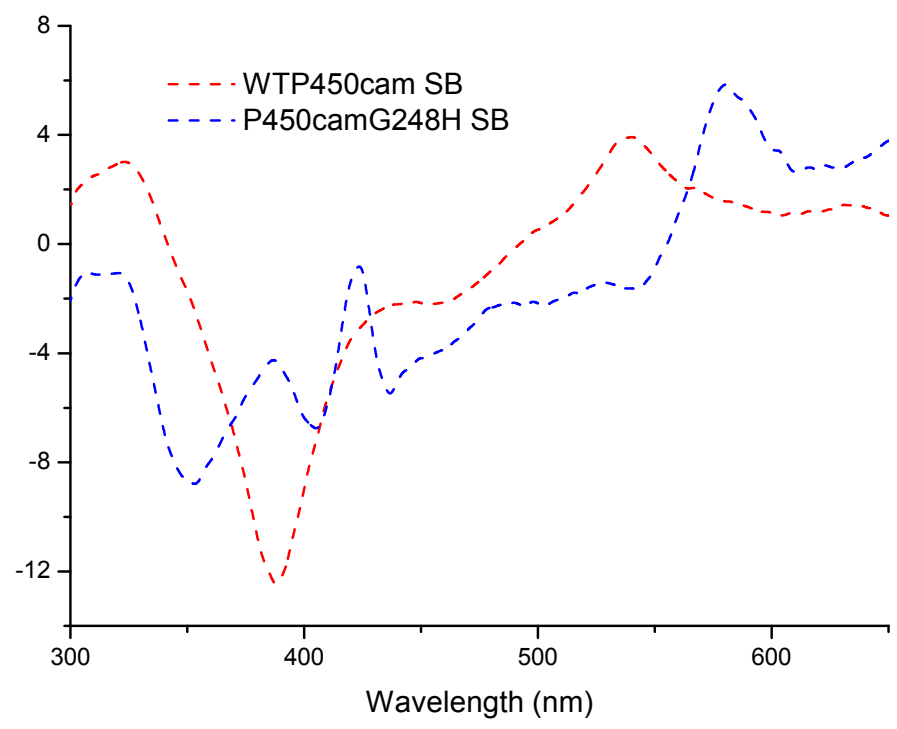

Figure 4.5 CD spectra of both substrate-free (A) and substrate-bound (B) forms of the WT and G248H mutant of cytochrome P450cam. 


\subsubsection{Electrochemical study}

Derived from Figure 4.6, the redox potential for G248H mutant was measured to be about $-92 \mathrm{mV}$, compared with $-135 \mathrm{mV}$ for wild type. There was some positive shift of the $\mathrm{Fe}^{3+} / \mathrm{Fe}^{2+}$ redox couple caused by the substitution of the distal glycine with a histidine residue. The redox potential change could be the result of the introduction of histidine into the distal heme pocket which increased the total charge near the heme group and made it easier to be reduced.

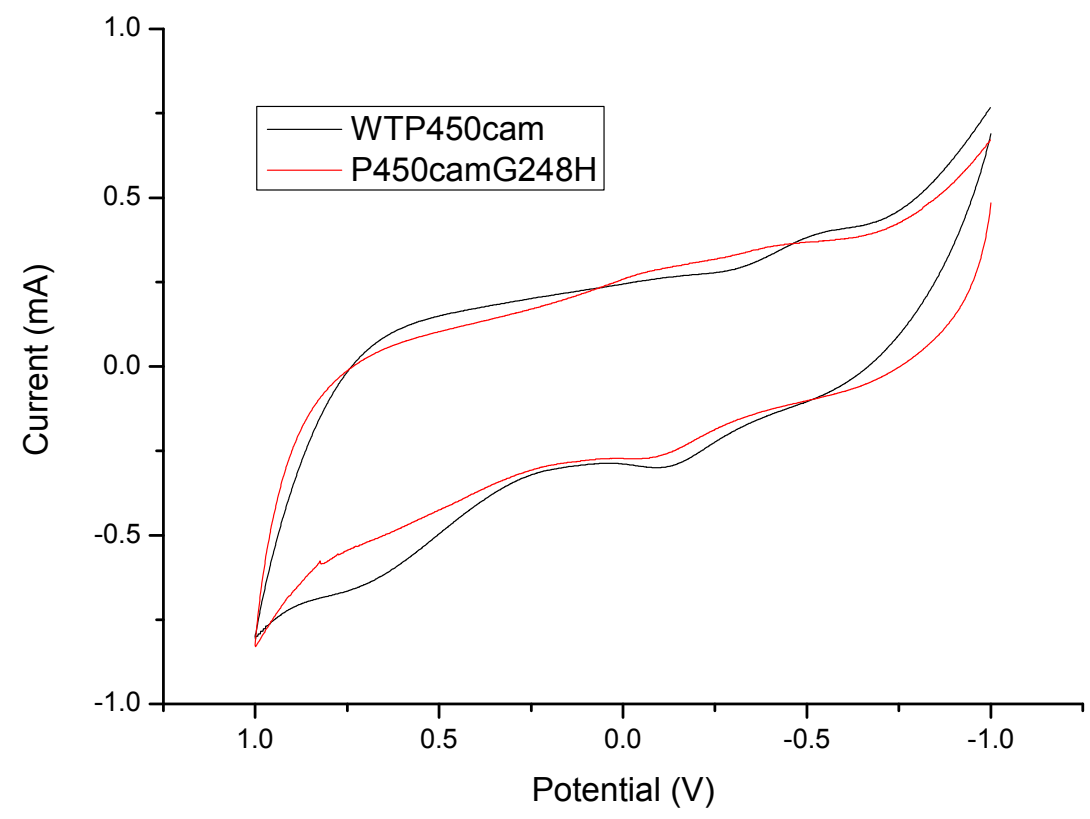

Figure 4.6 Cyclic voltammograms of P450cam and P450camG248H on a glass carbon electrode modified with SWNTs. Voltammograms were recorded between -1.0 and $+1.5 \mathrm{~V}$ at the scan rate of $1 \mathrm{~V} / \mathrm{sec}$ in $100 \mathrm{mM}$ phosphate buffer (pH7.4). 


\subsection{DISCUSSION AND CONCLUSION}

This work has demonstrated an intriguing model of heme-thiolate protein with a histidine serving as the $6^{\text {th }}$ axial ligand at the distal side of the heme group. Up to date, there are several proteins reported to possess such Cys-His coordination. In nature, this ligand set has only been seen in cystathionine $\beta$-synthase (32) and Rhodovulum sulfidophilum SoxAX enzyme (33). Also, a few hemoproteins have been engineered to introduce the distal histidine ligation or proximal cysteinate ligation in the heme pocket, such as the M80C variant of cytochrome $c$ (34), A264H variant of cytochrome P450BM3 (23), and H39C mutant of cytochrome b5 (35). But in all these cases, the proximal cysteinate ligation was only observed in the ferric but not the ferrous oxidation state. Upon reduction the proximal thiolate is either protonated or replaced by an alternative coordinating group except for the P450BM3 A264H mutant which was not even able to be completely reduced and form any significant amount of $\mathrm{CO}$ complex. We have presented here the first example of hemoprotein with a Cys-Fe-His ligand set which is preserved in various oxidation states.

In the electronic absorption spectroscopic studies, the UV-visible spectra of the G248H mutant of P450cam displayed features that are typical of six-coordinate, low-spin heme complexes in the absence of substrate. The Soret band of the camphor-free mutant was shown red shifted from $417 \mathrm{~nm}$ to $425 \mathrm{~nm}$, similar to what was observed in the imidazole-coordinated wild type protein and cytochrome P450BM3 A264H mutant, 
indicating the heme iron ligation is complete with distal water ligand replaced by histidine, a stronger field ligand. Interestingly the significant spin and coordination state changes that occur in wild type P450cam upon camphor binding were not observed in the mutant, and the UV spectra of G248H mutant in the absence and presence of camphor were virtually indistinguishable. This implied the substrate binding was perturbed in the variant, and camphor was not able to displace the axial heme ligand and alter the heme environment in the case of the wild type protein. Because of the complete axial ligation, it made the mutant unable to bind any ligands. This was confirmed by the resonance Raman spectroscopic spectra in which the ferric G248H variant showed LS, 6C with His and Cys as the distal and proximal ligand respectively, regardless of whether the substrate camphor is bound or not.

Comparing the ferrous form of the imidazole complex of wild type, the G248H mutant was different not only in its Soret band maximum but also in the $\mathrm{Q}$ band region. The mutant displayed two $\alpha$ and $\beta$ bands in the ferrous form, which was strong evidence of six coordination of the heme iron. However as for the imidazole complex of wild type protein, it showed only one band like the wild type and many other hemoproteins, indicating the preference of a five-coordination heme upon reduction. Intriguingly, in the study of the P450BM3 A264H mutant, a parallel variant of G248H, the distal ligand remained firmly bound on reduction and CO was not able to displace the His264 ligand. In $\mathrm{G} 248 \mathrm{H}$ mutant, its $\mathrm{CO}$ complex exhibited the characteristic Soret band at around 
$450 \mathrm{~nm}$ as the wild type, strongly indicating the preserved thiolate ligation in its ferrous form. Also, the RR spectra displayed distinct features of the mutant in its ferrous and $\mathrm{CO}$ complexes. The $v_{4}$ band of the ferrous G248H shifted to 1366 and $1360 \mathrm{~cm}^{-1}$, respectively for the substrate-free and substrate-bound forms, which were not in the region of either 5C cysteine proximal ligation $\left(1340-1348 \mathrm{~cm}^{-1}\right)$ or $5 \mathrm{C}$ histidine proximal ligation $\left(1350-1358 \mathrm{~cm}^{-1}\right)(36,37)$. This suggests that upon reduction, part of the histidine ligand was lost, resulting in a mixture of $5 \mathrm{C}$ and $6 \mathrm{C}$ coordination. Once $\mathrm{CO}$ was introduced, $\mathrm{CO}$ could partially bind to ferrous heme iron, but not dominantly.

According to the far-UV CD spectra, the secondary structure of the protein was not affected by the substitution of G248 with a histidine. However, there were indeed distinct changes in the tertiary structure around the heme pocket in the mutant compared with that of the wild type. The CD spectra showed markedly in different pattern in the presence and absence of camphor, especially in the substrate-bound form, which implied more significant conformational change. Except for the shift of Soret band, a set of split peaks was observed, indicative of strong six-coordinate ligation. Thus the binding of camphor makes the distal pocket more compact like the wild type and results in a stronger histidine ligation to the heme iron.

Taken together, the data of the various spectroscopic studies have demonstrated that the substitution of G248 with a histidine induces conformational change in the active site, yeilding a histidine ligation to the heme iron and alteration of the access of the substrate 
camphor to the heme prosthetic group. When the variant is reduced and CO is introduced, the proximal thiolate ligation remained intact while part of the distal histidine was displaced. This is quite different from other reported proteins with such Cys-Fe-His ligation set, whether it is naturally occurring or exogenously introduced. The thiolate ligand tends to be lost by protonation or displaced by another coordination group in the reduced form. This phenomenon has also been reported in many other hemoproteins with thiolate ligation in their ferric forms such as CO-sensing CooA protein (38), H93C mutant of myoglobin (Mb) (39), H175C mutant of cytochrome $c$ peroxidase (40) and inactive forms of cytochrome P450 (P420) and chloroperoxidase (C420) (41, 42). Except for CooA, which has been reported that the proximal ferrous ligand switches from Cys75 to His77, other hemoproteins has been proposed to use neutral cysteine thiol as the possible ligand (43) or the proximal cysteinate ligand could be replaced by a nearby histidine residue.

For instance, in the study of heme iron coordination of $\mathrm{C} 420$, it was suggested that ferric C420 appeared to retain a thiolate proximal ligand but not in its ferrous form with the distal histidine serving as a potential ligand to the heme iron. It has also been proposed in the probe of ferrous P420 that histidine ligation might be involved. All the hypotheses imply that histidine serves as an axial ligand to heme iron more readily than cysteinate in the hemoproteins. However, our results failed to support these hypotheses. Our study unequivocally demonstrated that the endogenous thiolate ligand is retained in 
all oxidation states in our $\mathrm{G} 248 \mathrm{H}$ mutant. It is evident that with both His and Cys ligated to the heme iron at the ferric state, it is the His rather than the Cys that would fall of up reduction and $\mathrm{CO}$ binding, suggesting the cysteinate ligation is more stable in this case, i.e. the iron-sulfur bond is stronger than the iron-nitrogen bond.

The crystal structural study of wild type P450cam complexed with imidazole has shown that the imidazole group was bound perpendicular to the plane of the heme (44). In the P450BM3 variant $\mathrm{A} 264 \mathrm{H}$, the angle between the plane of heme and imidazole ring of His-264 is almost exactly $90^{\circ}$ and the imidazole is orientated at about $45^{\circ}$ to the pyrrole nitrogens to minimize unfavorable interactions.(23) Therefore, we propose here that in the P450cam G248H mutant, the binding of the hisitidine ligand involves coordination of the imidazole group to the heme iron in a similar way to the two models mentioned above.

We conclude from the data presented in this work that the P450cam G248H mutant is the first model of complete axial coordination with cysteinate and histidine serving as the ligands to the heme iron, in which the thiolate ligation remains intact upon reduction and $\mathrm{CO}$ binding. We also propose that for hemoproteins with endogenous cysteine ligation, the thiolate ligand would not be easily displaced by another ligand provided by nearby residues or solvent and that cysteine serves as a stronger ligand than histidine. The study of this "locking" heme model could lead to the promising development of a diagnostic spectroscopic method for thiolate-ligated hemoproteins to recognize such 
coordination when it occurs elsewhere in nature or in other pertinent engineered protein systems.

\subsection{REFERENCES}

1. Sigel, A., Sigel, H., and Sigel, R. K.O. (2007) The ubiquitous roles of cytochrome P450 proteins in Metal Ions in Life Sciences (Sigel, A., Sigel, H., and Sigel, R. K.O. , Ed.), Wiley, Chichester.

2. Guengerich, F. P. (1991) Reactions and Significance of Cytochrome P-450 Enzymes, Journal of Biological Chemistry 266, 10019-10022.

3. Ortiz de Montellano, P. R. (2005) Cytochrome P450: structure, mechanism, and biochemistry, 3 ed., Kluwer Academic/Plenum Publishers, New York.

4. $\quad$ Sono, M. R., M. P.; Coulter, E. D.; and Dawson, J. H. (1996) Heme-Containing Oxygenases, Chemical Reviews 96, 2841-2847.

5. Sono, M., Roach, M. P., Coulter, E. D., and Dawson, J.H. (1996) Heme-Containing Oxygenases, Chemical Reviews 96, 2841-2847.

6. Joo, H., Lin, Z. L., and Arnold, F. H. (1999) Laboratory evolution of peroxide-mediated cytochrome P450 hydroxylation, Nature 399, 670-673.

7. Nordblom, G. D., White, R. E. and Coon, M. J. (1976) Studies on hydroperoxide-dependent substrate hydroxylation by purified liver microsomal cytochrome P450, Arch. Biochem. Biophys. 175, 524-533.

8. Hrycay, E. G., Gustafsson, J.-A., Ingelman-Sundberg, M. and Ernster, L. (1975) Sodium periodate, sodium chlorite, organic hydroperoxides and hydrogen peroxide as hydroxylating agents in steroid hydroxylation reactions catalyzed by partially purified cytochrome P450, Biochem. Biophys. Res. Commun. 66, 209-216.

9. Cirino, P. C., and Arnold, F. H. (2003) A self-sufficient peroxide-driven hydroxylation biocatalyst, Angew. Chem. Int. Ed. 42, 3299-3301. 
10. Shoji, O., Fujishiro, T., Nakajima, H., Kim, M., Nagano, S., Shiro, Y., Watanabe, Y. (2007) Hydrogen Peroxide-Dependent Monooxygenations by Tricking the Substrate Recognition of Cytochrome P450BS $\beta$, Angew. Chem. Int. Ed. 46, 3656-3659.

11. Cirino, P. C., and Arnold, F. H. (2002) Regioselectivity and activity of cytochrome P450 BM-3 and mutant F87A in reactions driven by hydrogen peroxide, Advanced Synthesis \& Catalysis 344, 932-937.

12. Davydov, R. M., Ledenev, A.N. (1981) Activation mechanism of molecular oxygen by cytochrome P-450, Biofizika 26, 1096.

13. Tanaka, M., Ishimori, K., Mukai, M., Kitagawa, T., and Morishima, I. (1997) Catalytic activities and structural properties of horseradish peroxidase distal His42-> Glu or Gln mutant, Biochemistry 36, 9889-9898.

14. Howes, B. D., RodriguezLopez, J. N., Smith, A. T., and Smulevich, G. (1997) Mutation of distal residues of horseradish peroxidase: Influence on substrate binding and cavity properties, Biochemistry 36, 1532-1543.

15. Newmyer, S. L., and Demontellano, P. R. O. (1995) Horseradish-peroxidase His-42 -> Ala, His-42 ->Val, and Phe-41 ->Ala mutants - Histidine Catalysis and Control of Substrate Access to the Heme Iron, J. Biol. Chem. 270, 19430-19438.

16. Erman, J. E., Vitello, L. B., Miller, M. A., Shaw, A., Brown, K. A., and Kraut, J. (1993) Histidine 52 is a critical residue for rapid formation of cytochrome c peroxidase compound I, Biochemistry 32, 9798-9806.

17. Poulos, T. L., and Kraut, J. (1980) THE STEREOCHEMISTRY OF PEROXIDASE CATALYSIS, J. Biol. Chem. 255, 8199-8205.

18. Poulos, T. L., Finzel, B. C., and Howard, A. J. (1987) High-Resolution Crystal-Structure of Cytochrome-P450cam, Journal of Molecular Biology 195, 687-700.

19. Schlichting, I., Berendzen, J., Chu, K., Stock, A. M., Maves, S. A., Benson, D. E., Sweet, B. M., Ringe, D., Petsko, G. A., and Sligar, S. G. (2000) The catalytic pathway of cytochrome P450cam at atomic resolution, Science 287, 1615-1622. 
20. Limburg, J., LeBrun, L. A., and de Montellano, P. R. O. (2005) The P450(cam) G248E mutant covalently binds its prosthetic heme group, Biochemistry 44, 4091-4099.

21. Joyce, M. G., Girvan, H. M., Munro, A. W., and Leys, D. (2004) A single mutation in cytochrome P450BM3 induces the conformational rearrangement seen upon substrate binding in the wild-type enzyme, J. Biol. Chem. 279, 23287-23293.

22. Girvan, H. M., Marshall, K. R., Lawson, R. J., Leys, D., Joyce, M. G., Clarkson, J., Smith, W. E., Cheesman, M. R., and Munro, A. W. (2004) Flavocytochrome P450BM3 mutant A264E undergoes substrate-dependent formation of a novel heme iron ligand set, J. Biol. Chem. 279, 23274-23286.

23. Girvan, H. M., Seward, H. Ed., Toogood, H. S., Cheesman, M. R., Leys, D. and Munro, A. W. (2007) Structural and Spectroscopic Characterization of P450 BM3 Mutants with Unprecedented P450 Heme Iron Ligand Sets, J. Biol. Chem. 282, $564-572$.

24. Paul, K. G., Theorell, H., Akesson, A. (1953) The Molar Light Absorption of Pyridine Ferroprotoporphyrin (Pyridine Haemochromogen), Acta Chem. Scand. 7, 1284-1287.

25. Berry, E. A., and Trumpower, B. L. (1987) Simultaneous Determination of Hemes-a, Hemes-b, and Hemes-c from Pryidine Hemochrome Spectra, Analytical Biochemistry 161, 1-15.

26. Dawson, J. H., Andersson, L. A. and Sono, M. (1982) Spectroscopic Investigations of Ferric Cytochrome P-450-CAM Ligand Complexes, J. Biol. Chem. 257, 3606-3617.

27. Spiro, T. G., Stong, J. D., and Stein, P. (1979) J. Am. Chem. Soc. 101, 2648-2655.

28. Andersson, L. A., Mylrajan, M., Sullivan, E. P., Jr., and Strauss, S. H. . (1989) J. Biol. Chem. 264, 19099-19102.

29. Lou, B. S., Snyder, J. K., Marshall, P., Wang, J. S., Wu, G., Kulmacz, R. J., Tsai, A. L., and Wang, J. (2000) Biochemistry 39, 12424-12434.

30. Spiro, T. G., Kozlowski, P. (2001) Acc. Chem. Res. 34, 137-144. 
31. Vogel, K. M., Kozlowski, P.M., Zgierski, M.Z., Spiro T.G. (2000) Inorg. Chim. Acta. 297, 11-17.

32. Meier, M., Janosik, M., Kery, V., Kraus, J. P., and Burkhard, P. (2001) Structure of human cystathionine $\beta$-synthase: a unique pyridoxal 5'-phosphate-dependent heme protein., EMBO Journal 20, 3910-3916.

33. Cheesman, M. R., Little, P. J., and Berks, B. C. (2001) Novel Heme Ligation in a c-type Cytochrome Involved in Thiosulfate Oxidation:EPR and MCD of SoxAX from RhodoVulum sulfidophilum, Biochemistry 40, 10562-10569.

34. Raphael, A. L. a. G., H. B. (1991) Semisynthesis of Axial-Ligand (Position 80) Mutants of Cytochrome c, J. Am. Chem. Soc. 113, 1038-1040.

35. Wang, W.-H., Lu, J-X, Yao, P., Xie, Y., Huang, Z-X. (2003) The distinct heme coordination environments and heme-binding stabilities of His39Ser and His39Cys mutants of cytochrome b5, Protein Eng. 16, 1047-1054.

36. Anzenbacher, P., Evangelista-Kirkup, R., Schenkman, J., Spiro, T. G. (1989) Influence of Thiolate Ligation on the Heme Electronic-Structure in Microsomal Cytochrome-P450 and Model Compounds - Resonance Raman-Spectroscopic Evidence, Inorganic Chemistry 28, 4491-4495.

37. Wang, J., Caughey, W. S., Rousseau, D. L. (1996) Resonance Raman Scattering: a Probe of Heme Protein-bound Nitric Oxide., in Methods in Nitric Oxide Research (Feelisch, M., Stamler, J., Ed.), pp 427-454, J. Wiley, Chichester ; New York.

38. Nakajima, H., Nakagawa, E., Kobayashi, K., Tagawa, S., and Aono, S. (2001) Ligand-switching intermediates for the CO-sensing transcriptional activator CooA measured by pulse radiolysis, Journal of Biological Chemistry 276, 37895-37899.

39. Adachi, S., Nagano, S., Ishimori, K., Watanabe, Y., Morishima, I., Egawas, T., Kitagawa, T., Makino, R. (1993) Roles of Proximal Ligand in Heme Proteins: Replacement of Proximal Histidine of Human Myoglobin with Cysteine and Tyrosine by Site-Directed Mutagenesis as Models for P-450, Chloroperoxidase, and Catalase, Biochemistry 32, 241-252. 
40. Sigman, J. A., Pond, A. E., Dawson, J. H., and Lu, Y. (1999) Engineering cytochrome $\mathrm{c}$ peroxidase into cytochrome P450: A proximal effect on heme-thiolate ligation, Biochemistry 38, 11122-11129.

41. Martinis, S. A., Blanke, S. R., Hager, L. P., Sligar, S. G., Hoa, G. H. B., Rux, J. J., and Dawson, J. H. (1996) Probing the heme iron coordination structure of pressure-induced cytochrome P420cam, Biochemistry 35, 14530-14536.

42. Blanke, S. R., Martinis, S. A., Sligar, S. G., Hager, L. P., Rux, J. J., Dawson, J. H. (1996) Probing the Heme Iron Coordination Structure of Alkaline Chloroperoxidase, Biochemistry 35, 14537-14543.

43. Perera, R., Sono, M., Sigman, J. A., Pfister, T. D., Lu, Y., and Dawson, J. H. (2003) Neutral thiol as a proximal ligand to ferrous heme iron: Implications for heme proteins that lose cysteine thiolate ligation on reduction, Proc. Natl. Acad. Sci. 100, 3641-3646.

44. Verras, A., Alian, A., and de Montellano, P. R. O. (2006) Cytochrome P450 active site plasticity: attenuation of imidazole binding in cytochrome P450(cam) by an L244A mutation, Protein Engineering Design \& Selection 19, 491-496. 


\section{CHAPTER V. PROTEIN ENGINEERING OF MYOGLOBIN WITH THIOLATE PROXIMAL LIGATION}

\subsection{SUMMARY}

Myoglobin has been used as a model in the investigations of protein folding, ligand binding and conformational transitions of heme-containing proteins. Many studies of heme-imidazole proteins including myoglobin have been carried out to replace the proximal axial histidine ligand with a cysteine residue to gain the heme-thiolate protein structural character. Although some of the protein variants achieved the iron-thiolate ligation in their ferric forms, the iron-thiolate ligand failed to retain upon reduction. Therefore, to explore the possibility of converting myoglobin to a heme-thiolate protein in all oxidation states, I tried to mimic the protein environment of both cytochrome P450cam and chloroperoxidase respectively in the proximal side of sperm whale myoglobin by replacing eight amino acids around the iron-histidine ligand. UV-visible spectroscopic studies were performed to examine the axial ligation in the wild type and mutant proteins of $\mathrm{Mb}$. Neither the P450 mimic nor the CPO mimic showed the characteristic Soret absorption maxima around $450 \mathrm{~nm}$ when reduced in the presence of carbon monoxide, suggesting the thiolate ligation was not reserved in the ferrous forms. Considering the possible interference of distal histidine as a competitive candidate ligand to the heme iron, the His64 was substituted with an alanine and glutamic acid based on 
comparisons with the structure of $\mathrm{P} 450$ and $\mathrm{CPO}$, respectively. The optical spectral properties showed no difference. It happened again in the further mutation by replacing the His 97 which is located in the proximal vicinity with an alanine.

\subsection{INTRODUCTION}

Myoglobin is a monomeric heme protein found mainly in muscle tissues where it acts as an intracellular primary storage site for oxygen by reversibly binding oxygen molecules (1). This protein has been the target of extensive biochemical and biophysical studies for many years because of its physiological importance, availability and simplicity compared with other bulky heme proteins. It serves as a prototype for elucidating the roles of critical active site residues in the interactions of hemoproteins with small molecules such as dioxygen, carbon monoxide, and cyanide (2-5). In myoglobin, there is a conserved histidine residue (His93) serving as the proximal axial ligand to the heme iron, anchoring the heme group in the globin, and a conserved hisitidine residue (His64) in the distal pocket, conferring a large measure of specificity for $\mathrm{O}_{2}(6)$. Both of these histidine residues are important in terms of protein structure and function. Quite a few studies have been dedicated to examine their roles in the active site of myoglobins from various sources by site-directed mutagenesis in the last few decades $(7-11)$ 
Cysteine plays a key role as a metal ligand in metalloproteins, especially in heme-containing proteins. A distinctive feature of heme-thiolate proteins is a Soret absorption band at around $450 \mathrm{~nm}$ in the $\mathrm{CO}$ difference spectrum of the reduced forms. The anionic cysteinate that coordinates to the heme iron in heme-thiolate proteins has been claimed to serve as a strong electron donor to facilitate the heterolytic O-O bond cleavage and the formation of $\mathrm{Cpd}$, the highly active reaction intermediate responsible for the catalytic reactions $(12,13)$. To clarify the critical role of the anionic axial ligands of heme proteins on the $\mathrm{O}-\mathrm{O}$ bond scission reaction and the contributions of proximal Cys ligation to the functional and catalytic properties of cytochrome P450 and CPO, the proximal histidine 93 of human myglobins and horse heart myoglobin has been replaced with a cysteine residue $(9,10)$. The $\mathrm{Mb} \mathrm{H} 93 \mathrm{C}$ mutants resulted in altered axial ligations analogous to those of $\mathrm{P}-450$ or $\mathrm{CPO}$. The cysteine coordination to the ferric heme iron is confirmed by various biophysical methods, with the mutants showing almost identical spectroscopic properties to those of heme-thiolate proteins. The Mb H93C mutants were revealed to be in the ferric high-spin, five-coordinate state with the proximal cysteine ligated to the heme iron (9). Therefore, it is proved that the axial ligand of the heme iron is responsible for the spectroscopic features of hemoproteins, which has been indicated by various hemoproteins and their ligand-bound complexes.

For catalytic activities, the cysteine must be deprotonated to cysteinate throughout the reaction cycle as the iron changes oxidation state from ferric to ferrous and ferryl 
states. The role of the anionic axial ligands on the $\mathrm{O}-\mathrm{O}$ bond cleavage is examined by reactions utilizing peroxides. The results of $\mathrm{Mb} \mathrm{H93C}$ mutants showed that the thiolate ligand enhanced the heterolytic $\mathrm{O}-\mathrm{O}$ bond scission of the oxidant. This mutant also displayed about a 5-fold increase in epoxidation activity (9). Although the ferric forms of the mutants showed iron-cysteine ligation, upon reduction of the H93C variants with sodium dithionite under a $\mathrm{CO}$ atmosphere, the $\mathrm{CO}$ complexes displayed a similar spectrum to that of wild type $\mathrm{Mb}$, the typical heme-imidazole protein, with the Soret band at around $420 \mathrm{~nm}$ instead of $450 \mathrm{~nm}$. Thus, it is unlikely that the cysteine thiolate remains as the proximal ligand to the ferrous heme iron. The spectroscopic study of the mutants confirms that in $\mathrm{Mb} \mathrm{H93C} \mathrm{the} \mathrm{distal} \mathrm{histidine} \mathrm{is} \mathrm{coordinated} \mathrm{to} \mathrm{the} \mathrm{heme} \mathrm{iron,} \mathrm{despite} \mathrm{the}$ suggestion of a neutral thiol serving as the proximal ligand upon reduction $(10,14)$. On the basis of the H93C single mutant, several studies have been carried out to replace the distal histidine with a glycine, valine or isoleucine as well. The ferric state of these variants closely resembled the spectroscopic properties of the corresponding ferric cytochrome P450. However, the ferrous and ferrous-CO complexes of these variants remained virtually the same as for the $\mathrm{H} 93 \mathrm{C}$ single mutant. According to these results, it is apparent that while simple mutagenesis modifications of the $\mathrm{Mb}$ active site can mimic the characteristics of ferric cytochrome $\mathrm{P} 450$ or $\mathrm{CPO}$, the reproduction of the spectroscopic properties of their ferrous forms would require more subtle modifications though, which is the ultimate goal of this study. 
Sperm whale myoglobin $(\mathrm{Mb})$, a single-chain globin protein of 154 amino acids has been chosen because it reprents one of the most extensively studied heme-containing proteins by biochemical, biophysical and spectroscopic techniques. It was the first protein whose crystal structure was determined to high resolution by $\mathrm{x}$-ray crystallographic analysis in 1960 (15). Sperm whale myoglobin is composed of eight $\alpha$ helices (labeled A-H in Fig. 5.1) that form an amphipathic pocket with the heme prosthetic group bound between the helices. The heme iron is coordinated to the protein via a histidine ligand located in the F helix at the proximal site. The majority of other heme-protein contacts are hydrophobic except for the two solvent- exposed propionate side chains of the heme group that interact electrostatically with the protein residues around the prosthetic group.

Figure 5.2 illustrates the active site structure of sperm whale myoglobin with histidine residues in both proximal and distal sides along with several important amino acids in the proximal helix $\mathrm{F}$ from Leu89 to His97, with His 93 in the middle. To engineer sperm whale myoglobin into a heme-thiolate protein model in various oxidation states, all eight proximal residues around the axial ligand were replaced by the corresponding amino acids in P450cam and $\mathrm{CPO}$, resulting in the P450 mimic of $\mathrm{Mb}$ $(\mathrm{MbP})$ and $\mathrm{CPO}$ mimic of $\mathrm{Mb}(\mathrm{MbO})$. Furthermore, the distal histidine was substituted with an alanine and glutamic acid, respectively, for $\mathrm{MbP}$ and MbO. Finally, as the distal H97 that could be a potential competitive ligand to the heme iron in the ferrous forms of the $\mathrm{Mb}$ variants was also replaced. UV-visible spectroscopic studies have been carried out 
to examine the optical properties and structural characteristics of the generated mutants of sperm whale myoglobin.

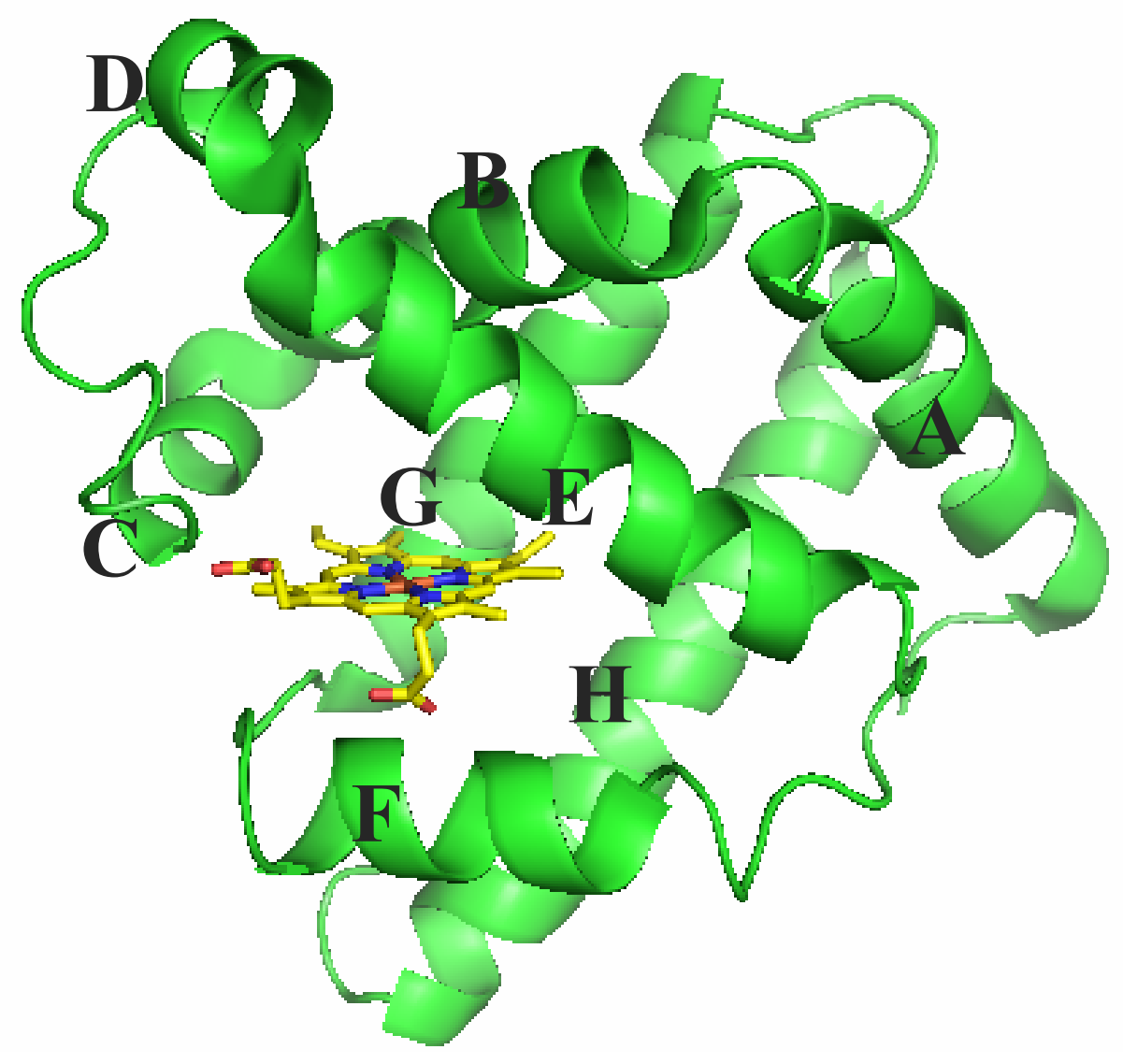

Figure 5.1 A ribbon diagram of the sperm whale myoglobin crystal structure showing the arrangement of eight $\alpha$ helices and the heme group (shown in yellow). This figure was generated from the crystal data (PDB code 1VXH) using PYMOL (6). 
A
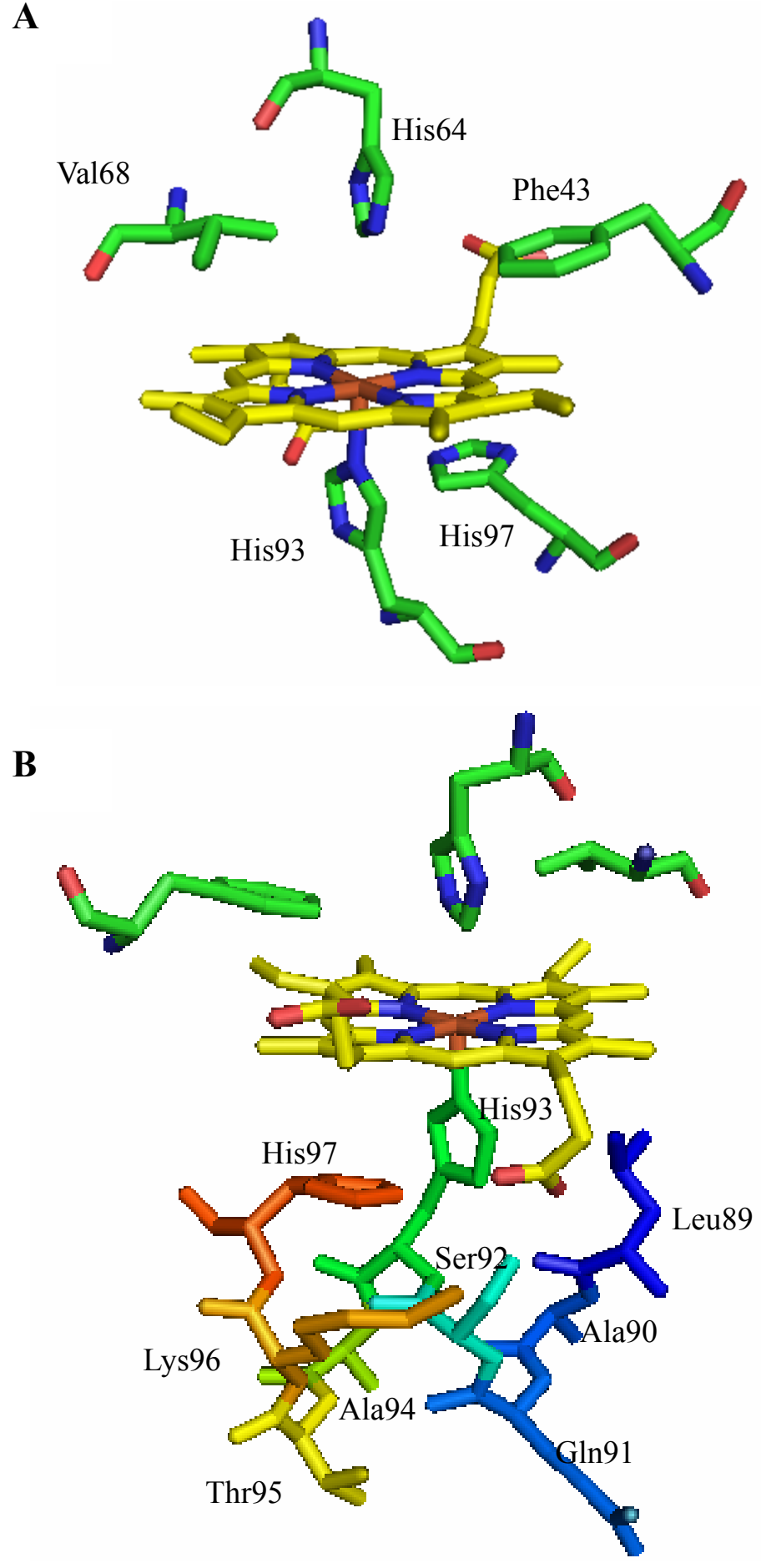

Figure 5.2 Crystal structure of sperm whale myglobin active site with some important amino acids around heme group (yellow) viewed from different angles. In (B) the proximal amino acids were colored in rainbow. The figures were generated from the crystal data (PDB code 1VXH) using PYMOL (1). 


\subsection{EXPERIMENTAL PROCEDURES}

\subsubsection{Bacterial strains, plasmids and DNA manipulations}

The bacterial strains used were E. coli NovaBlue and E. coli Rosetta II (DE3) from Novagen (Gibbstown, NJ). The used plasmid pMb122 containing wild type sperm whale myoglobin gene in a pUC19 vector is identical to pMb413a used by Springer et al. except for a correction in the coding sequence (N122D) due to a mistake in the original sequence (16). To increase the protein yield, the $\mathrm{Mb}$ gene was constructed into a T7-based expression vector, pET-30a $(+)$ from Novagen, at the sites of NdeI and KpNI by polymerase chain reactions and ligation reactions. Bacteria were grown in Luria-broth supplemented with antibiotic (kanamycin $100 \mu \mathrm{g} / \mathrm{ml}$ ) for DNA manipulation.

\subsubsection{Site-directed mutagenesis and protein expression.}

Table 5.1 Proximal sequence alignment of $\mathrm{Mb}$ and its $\mathrm{P} 450$ and $\mathrm{CPO}$ mimics

\begin{tabular}{lcccccccc}
\hline Protein & Proximal sequence & & & & \\
\hline WT Mb & L & A & Q & S & H93 & A & T & K \\
MbP (P450 mimic) & G & S & R & L & C93 & L & G & Q \\
MbO (CPO mimic) & S & R & A & P & C93 & P & A & L \\
\hline
\end{tabular}

The proximal sequence alignment of sperm whale $\mathrm{Mb}$ and its $\mathrm{P} 450$ and $\mathrm{CPO}$ mimics is listed in Table 5.1. The MbP mutant was prepared with the 5'-sense oligonucleotide 5'$\underline{\text { GGT }} \underline{\text { TCT }} \underline{\text { CGT }} \underline{\text { CTG }} \underline{\text { TGC }} \underline{\text { CTG }} \underline{\text { GGT }} \underline{\text { CAG }}$ CAT AAG ATC CCG ATC AA coding for GSRLCLGQHKIPI (P450mimic). The MbO mutant was obtained with the 5'-sense 


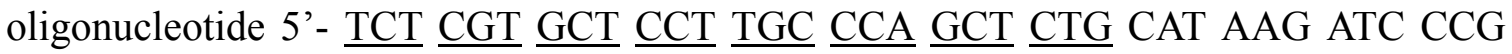
ATC AA coding for SRAPCPALHKIPI. Primers used for all other Mb variants are listed in appendix $\mathrm{C}$. The reconstituted plasmids containing $\mathrm{Mb}$ and the mutants were transformed into E. coli strain NovaBlue and verified by DNA sequencing (Macrogen, MD). Proteins were expressed in E. coli strain Rosetta II (DE3). Cells were grown on Luria broth supplemented with $100 \mathrm{mg} / \mathrm{L}$ of kanamycin at $37{ }^{\circ} \mathrm{C}$ and induced with $1 \mathrm{mM}$ IPTG when $\mathrm{OD}_{600}$ reashes around 1.2, followed by further growth for 4 hours prior to harvest. As for the mutants, the addition of $\delta$-aminolevulinic acid to the growth media is necessitated to facilitate holoenzyme production. The cells were then harvested and frozen at $-80{ }^{\circ} \mathrm{C}$.

\subsubsection{Protein purification}

Cells were lysed at $4^{\circ} \mathrm{C}$ in $25 \mathrm{mM}$ Tris- $\mathrm{HCl}$ buffer at $\mathrm{pH} 8.0$ containing $0.5 \mathrm{mM}$ DTT, 1 mM EDTA, $200 \mu \mathrm{M}$ PMSF, 40 U/ml Dnase I, 3 U/ml Rnase A, and lysozyme for overnight in a shaker. After freezing at $-80^{\circ} \mathrm{C}$ for the second time, the cells were thawed again followed by centrifugation and concentration using an Amicon cell with 10,000 Da cut-off ultrafiltration membrane. The protein solution was first applied on a fast flow DEAE Sepharose column equilibrated with the $10 \mathrm{mM}$ Tris- $\mathrm{HCl}$ buffer at $\mathrm{pH}$ 8.2. After initial wash, proteins were eluted with a linear $0-0.5 \mathrm{M} \mathrm{NaCl}$ gradient in the same buffer. All $\mathrm{Mb}$ mutants were expressed as apo-proteins and converted to holoproteins via heme reconstitution. Typically, hemin was added to the apo-protein drop wise which was 
mixing on a stirrer for overnight (17). After heme reconstitution, the protein sample was again applied onto a fast flow DEAE Sepharose column. The reddish fractions of correct molecular weight were pooled and concentrated using a Centriprep with an YM10 membrane for further purification by size exclusion chromatography. The buffer used for gel filtration was $0.1 \mathrm{M}$ potassium phosphate buffer at $\mathrm{pH}$ 7.0. Unless otherwise specified, all steps in the protein purification procedure were performed at $4{ }^{\circ} \mathrm{C}$.

\subsubsection{Electronic absorption spectroscopy}

The UV-visible spectra of the Mb and its mutants were recorded on a Varian Cary 300 Bio UV-visible spectrophotometer at room temperature. The ferrous proteins were prepared by the addition of excess amount of sodium dithionite and $\mathrm{CO}$ adducts were

obtained by CO gas bubbling. The buffer used for the absorption measurements was 100 $\mathrm{mM}$ potassium phosphate buffer at $\mathrm{pH}$ 7.0.

\subsection{RESULTS AND DISCUSSION}

There are two major groups of hemoprotein found in living organisms, namely "heme-thiolate proteins" and "heme-imidazole proteins". The difference between these two classes of proteins is that when carbon monoxide binds to the reduced forms, heme-thiolate proteins usually give a characteristic optical absorption spectrum with a prominent Soret peak at around $450 \mathrm{~nm}$, whereas the corresponding absorption spectrum of heme-imidazolate proteins shows a Soret peak at around $420 \mathrm{~nm}$. This work intended 

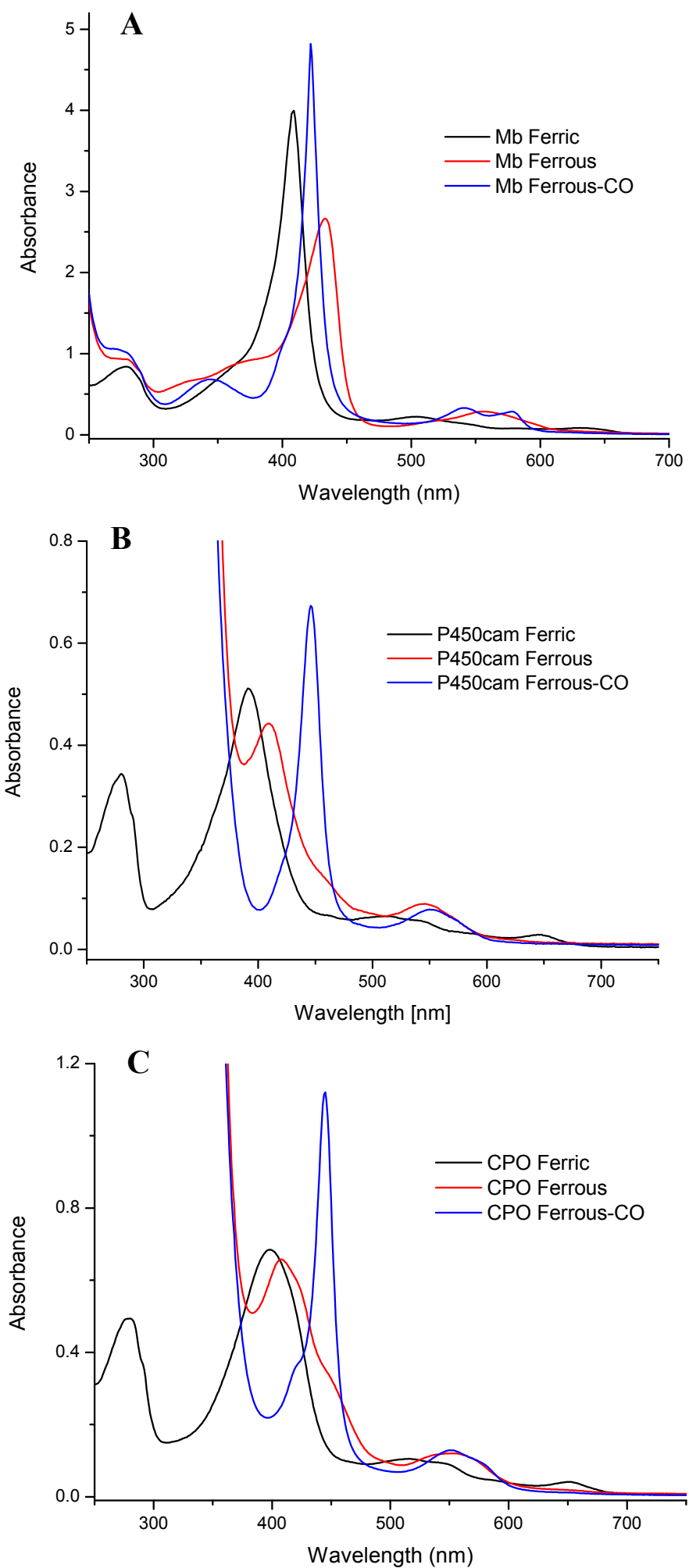

Figure 5.3. UV-visible spectra of wild type $\mathrm{Mb}(\mathbf{A})$, P450cam (B) and CPO (C) in various oxidation states 
to investigate the role of proximal ligands in heme-thiolate proteins by changing the amino acids at the proximal side of sperm whale myoglobin, a typical heme-imidazole protein, to that of $\mathrm{P} 450$ or $\mathrm{CPO}$.

First of all, wild type myoglobin, cytochrome P450cam and CPO were expressed and purified according to reported procedures (18-20). Figure 5.3 showed the UV-vis spectroscopic results of these proteins. In agreement with the literature, the ferrous-CO complexes of $\mathrm{Mb}$ displayed Soret absorption maximum at $420 \mathrm{~nm}$, while P450cam and $\mathrm{CPO}$ exhibited the Soret band at $450 \mathrm{~nm}$. By changing the proximal helix residues to mimic those of P450 and $\mathrm{CPO}$, the resulted mutants were expected to show spectral properties similar to such heme-thiolate proteins.

\subsubsection{Mb mutant: $\mathrm{MbP} / \mathrm{MbO}$}

Eight amino acids, at the heme proximal side of sperm whale $\mathrm{Mb}$, including His 93 have been replaced to mimic the protein environment of cytochrome P450cam and CPO, respectively. The mutants were named $\mathrm{MbP}$ and $\mathrm{MbO}$. The primers were designed as one containing all the mutations at the end and the other one having the gene sequence at the opposite direction. The PCR and self-ligation reactions were performed which directly produced the mutated recombinant plasmid with the facilitation of T4 polynucleotide kinase. Both of the mutants were over expressed in E. coli. Since they showed quite similar results, only the electronic absorption spectra of the P450 mimic were included. As shown in Fig. 5.4, the UV-vis spectra of Mb exhibited very similar results with those 
of wild type $\mathrm{Mb}$ with the Soret band of the ferrous heme-CO complex at $420 \mathrm{~nm}$, instead of $450 \mathrm{~nm}$ expected for Fe-thiolate ligation. This indicated that the thiolate sulfur might not be ligated to the heme iron upon reduction and $\mathrm{CO}$ binding. There could be other possible factors governing the spectral properties of the mutant, for instance the influence of the distal histidine. Therefore, the distal histidine was replaced by a glycine and glutamic acid in the P450 and CPO mimics, respectively.

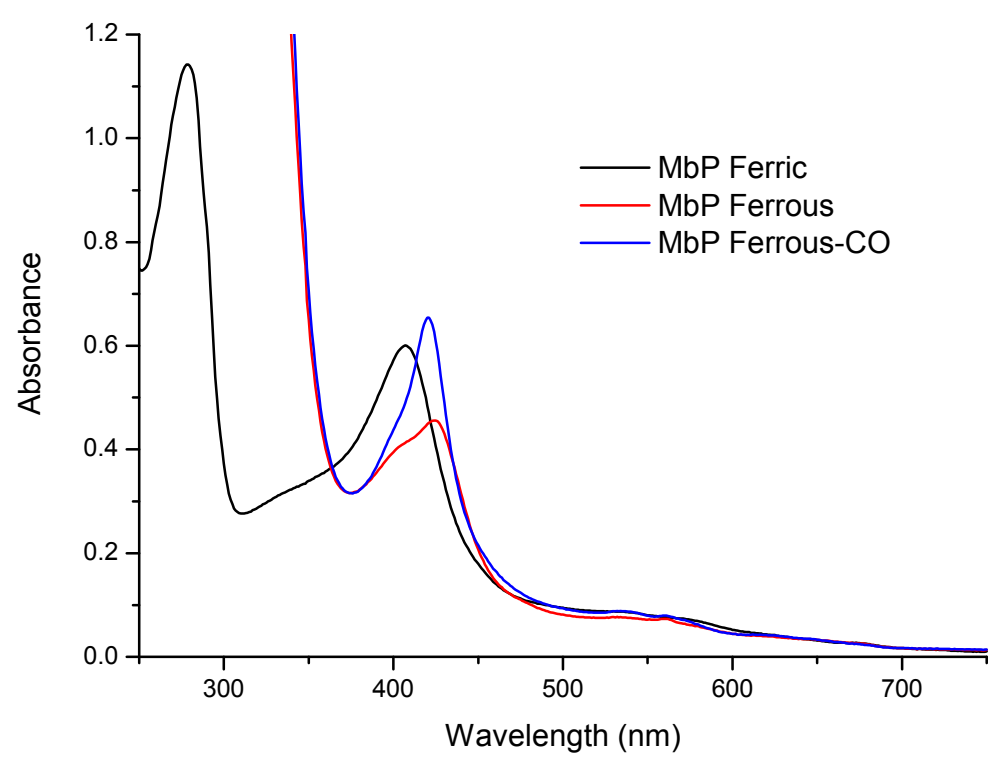

Figure 5.4 UV-visible spectra of MbP in various oxidation states. 


\subsubsection{Mb mutant: MbP H64G/MbO H64E}

To investigate the possible interference of the distal histidine, MbP H64G and MbO H64E mutants were obtained. Fig. 5.5 shows the UV-vis results of the MbP H64G mutant. Unexpectedly, the Soret peak of the ferrous- $\mathrm{CO}$ complex of this $\mathrm{Mb}$ mutant remained at around $420 \mathrm{~nm}$ (Table 5.2). Thus, further modifications are needed to achieve a thiolate proximal ligand in the $\mathrm{Mb}$ variant at reduced state.

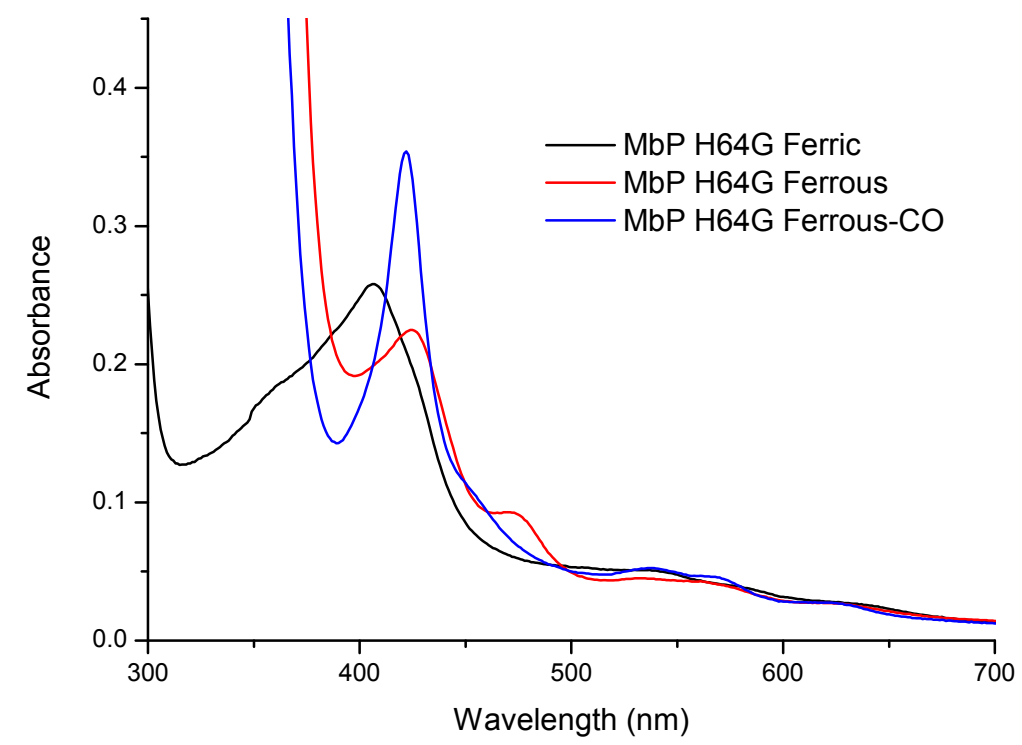

Figure 5.5 UV-visible spectra of MbP H64G in various oxidation states. 
Table 5.2 Wavelength and extinction coefficients of absorption maxima for P450cam, Mb, MbP, and MbP H64G.

\begin{tabular}{llll}
\hline Protein & Oxidation state & Soret band $(\mathrm{nm})$ & Visible bands $(\mathrm{nm})$ \\
\hline \multirow{4}{*}{ P450cam } & $\mathrm{Fe}^{3+}$ & $392(\varepsilon=102)(21)$ & 535,569 \\
& $\mathrm{Fe}^{2+}$ & 408 & 540 \\
& $\mathrm{Fe}^{2+-} \mathrm{CO}$ & $\mathbf{4 4 6}$ & 550 \\
\hline \multirow{3}{*}{$\mathrm{CPO}$} & $\mathrm{Fe}^{3+}$ & $398(\varepsilon=91.2)(22)$ & $516,550,650$ \\
& $\mathrm{Fe}^{2+}$ & 408 & 550 \\
& $\mathrm{Fe}^{2+-} \mathrm{CO}$ & $\mathbf{4 4 5}$ & 550 \\
\hline \multirow{3}{*}{$\mathrm{Mb}$} & $\mathrm{Fe}^{3+}$ & $408(\varepsilon=184)(11)$ & 502,631 \\
& $\mathrm{Fe}^{2+}$ & 433 & 556 \\
& $\mathrm{Fe}^{2+-} \mathrm{CO}$ & $\mathbf{4 2 2}$ & 541,578 \\
\hline \multirow{3}{*}{$\mathrm{MbP}$} & $\mathrm{Fe}^{3+}$ & 407 & 534,569 \\
& $\mathrm{Fe}^{2+}$ & 425 & 532,559 \\
& $\mathrm{Fe}^{2+-} \mathrm{CO}$ & $\mathbf{4 2 1}$ & $533,561,619$ \\
\hline \multirow{3}{*}{$\mathrm{MbP} \mathrm{H64G}$} & $\mathrm{Fe}^{3+}$ & 407 & 537,576 \\
& $\mathrm{Fe}^{2+}$ & 425 & 533,561 \\
& $\mathrm{Fe}^{2+-} \mathrm{CO}$ & $\mathbf{4 2 2}$ & $538,568,620$ \\
\hline
\end{tabular}

\subsubsection{Mb mutant: MbP H64G H97A/MbO H64E H97A}

Considering that the proximal histidine 97 is located in the helix F closely to the heme iron and the iron-histidine ligand (His93) (Fig. 5.2), it could be a possible competitor to interference the altered thiolate ligation in the proximal side of MbP H64G mutant. Therefore, it was replaced by an alanine, which is a good helix promoter, for both the P450 and CPO mimics. Unfortunately, the UV-vis results showed nothing different from the MbP H64G mutant (data not shown). In conclusion, the attempt to engineer 
myoglobin with a proximal thiolate ligand that can be retained in all oxidation states to achieve a P450-like variant was not successful. This difficulty was also reported by several previous studies, in which the proximal histidine was replaced by a cysteine but the thiolate ligation was lost upon reduction (23-26). It is apparent that the heme-thiolate proteins and heme-imidazole proteins have distinct structural and functional differences and it would take more than just mimicking the active site residues to convert one to another.

\subsection{REFERENCES}

1. Yang, F., and Phillips, G. N. (1996) Crystal structures of CO-, deoxy- and met-myoglobins at various $\mathrm{pH}$ values, $J$. Mol. Biol. 256, 762-774.

2. Brancaccio, A., Cutruzzolá, F., Allocatelli, C.T., Brunori, M., Smerdon, S.J., Wilkinson, A.J., Dou, Y., Keenan, D., Ikeda-Saito, M., and Brantley, R.E. (1994) Structural factors governing azide and cyanide binding to mammalian metmyoglobins, J. Biol. Chem. 269, 13843-13853.

3. $\quad$ Springer, B. A., Egeberg, K.D., Sligar, S.G., Rohlfs, R.J., Mathews, A.J., and Olson, J.S. (1989) Discrimination between oxygen and carbon monoxide and inhibition of autooxidation by myoglobin. Site-directed mutagenesis of the distal histidine, J. Biol. Chem. 264, 3057-3060.

4. Olson, J. S., and Phillips, J. N. (1996) Kinetic Pathways and Barriers for Ligand Binding to Myoglobin, J. Biol. Chem. 271, 17593-17596.

5. Dou, Y., Olson, J.S., Wilkinson, A.J., and Ikeda-Saito, M. (1996) Mechanism of Hydrogen Cyanide Binding to Myoglobin, Biochemistry 35, 7107-7113.

6. Springer, B. A., Sligar, S. G., Olson, J. S., Phllips, G. N. (1994) Mechanisms of ligand recognition in myoglobin, Chem. Rev. 94, 699-714. 
7. Egeberg, K. D., Springer, B. A., Martinis, S. A., Sligar, S. G., Morikis, D., and Champion, P. M. (1990) Alteration of sperm whale myoglobin heme axial ligation by site-directed mutagenesis, Biochemistry 29, 9783-9791.

8. Matsui, T., Ozaki, S., and Watanabe, Y. (1999) Formation and catalytic roles of compound I in the hydrogen peroxide-dependent oxidations by His64 myoglobin mutants, Journal of the American Chemical Society 121, 9952-9957.

9. Adachi, S., Nagano, S., Ishimori, K., Watanabe, Y., Morishima, I., Egawa, T., Kitagawa, T., and Makino, R. (1993) Roels of Proximal Ligand in Heme-proteins-Replacement of Proxmial Histidine of Human Myoblobin with Cysteine and Tyrosine by Site-directed Mutagenesis as Models for P-450, Chloroperoxidase, and Catalase, Biochemistry 32, 241-252.

10. Hildebrand, D. P., Ferrer, J. C., Tang, H.-L., Smith, M., Mauk, A. G. (1995) Trans Effects on Cysteine Ligation in the Proximal His93Cys Variant of Horse Heart Myoglobin, Biochemistry 34, 11598-11605.

11. Matsui, T., Nagano, S., Ishimori, K., Watanabe, Y., and Morishima, I. (1996) Preparation and reactions of myoglobin mutants bearing both proximal cysteine ligand and hydrophobic distal cavity: Protein models for the active site of P-450, Biochemistry 35, 13118-13124.

12. Ortiz de Montellano, P. R. (2005) Cytochrome P450: structure, mechanism, and biochemistry, 3 ed., Kluwer Academic/Plenum Publishers, New York.

13. Newcomb, M., Zhang, R., Chandrasena, R. E. P., Halgrimson, J. A., Horner, J. H., Makris, T. M., and Sligar, S. G. (2006) Cytochrome P450 Compound I, Journal of the American Chemical Society 128, 4580-4581.

14. Perera, R., Sono, M., Sigman, J. A., Pfister, T. D., Lu, Y., and Dawson, J. H. (2003) Neutral thiol as a proximal ligand to ferrous heme iron: Implications for heme proteins that lose cysteine thiolate ligation on reduction, Proc. Natl. Acad. Sci. $100,3641-3646$.

15. Kendrew, J. C., Dickerson, R. E., Strandberg, B. E., Hart, R. G., Davis, D. R., Phillips, D. C., and Shore, V. C. (1960) Structure of Myoglobin: A Three-Dimensional Fourier Synthesis at $2 \AA$ Resolution, Nature 185, 422-427. 
16. Phillips, G. N., Arduini, R. M., Springer, B. A., and Sligar, S. G. (1990) Crystal Structure of Myoglobin From a Synthetic Gene, Proteins-Structure Function and Genetics 7, 358-365.

17. Wagner, G. C., Perez, M., Toscano, W. A. Jr., and Gunsalus, I. C. (1981) Apoprotein Formation and Heme Reconstitution of Cytochrome P-450cam, $J$. Biol. Chem. 256, 6262-6265.

18. Springer, B. A., and Sligar, S. G. (1987) High-Level Expression of Sperm Whale Myoglobin in Escherichia-coli, Proc. Natl. Acad. Sci. U. S. A. 84, 8961-8965.

19. Morris, D. R., Hager, L. P. (1966) Chloroperoxidase: I. Isolation and Properties of the Crystalline Glycoprotein J. Biol. Chem. 241, 1763-1768.

20. Unger, B. P., Gunsalus, I. C., Sligar, S. G. (1986) Nucleotide-sequence of the Pseudomonas-putida Cytochrome P450-cam Gene and Its Expression in Escherichia-coli, J. Biol. Chem. 261, 1158-1163.

21. Yu, C.-A., Gunsalus, I. C., Katagiri, M., Suhara, K., and Takemori, S. (1974) Cytochrome P-450cam: I. Crystallization and Properties J. Biol. Chem. 249, 94-101.

22. Chiang, R., Makino, R., Spomer, W. E., Hager, L. P. (1975) Chloroperoxidase. P-450 type absorption in the absence of sulfhydryl groups, Biochemistry 14, 4166-4171.

23. Sigman, J. A., Pond, A. E., Dawson, J. H., and Lu, Y. (1999) Engineering cytochrome c peroxidase into cytochrome P450: A proximal effect on heme-thiolate ligation, Biochemistry 38, 11122-11129.

24. Liu, Y., Moenne-Loccoz, P., Hildebrand, D. P., Wilks, A., Loehr, T. M., Mauk, A. G., and Ortiz de Montellano, P. R. (1999) Replacement of the Proximal Histidine Iron Ligand by a Cysteine or Tyrosine Converts Heme Oxygenase to an Oxidase, Biochemistry 38, 3733-3743.

25. Raphael, A. L. a. G., H. B. (1991) Semisynthesis of Axial-Ligand (Position 80) Mutants of Cytochrome c, J. Am. Chem. Soc. 113, 1038-1040. 
26. Wang, W., Lu, J., Yao, P., Xie, Y., and Huang, Z. (2003) The distinct heme coordination environments and heme-binding stabilities of His39Ser and His39Cys mutants of cytochrome b5, Protein Engineering 16, 1047-1054. 


\section{APPENDIX A}

\section{Sequences of P450cam and pET-30a $(+)$ Vector}

\section{General information}

The cytochrome P450cam protein includes 414 amino acids.

Figure A.1 lists the amino acid sequence of full-length, wild-type cytochrome P450cam.

Figure A.2 lists the complete nucleotide sequence of the 1578-bp PstI-Hind111 fragment, including the wild-type cytochrome P450cam structural gene from Pseudomonas putida, $155 \mathrm{bp}$ of $5^{\prime}$ flanking sequence, and $175 \mathrm{bp}$ of 3' DNA.

Figure A.3 lists the nucleotide sequence of vector pET-30a $(+)$ from Novagen.

Figure A.4 displays a map of plasmid pET-30a (+)_P450cam, which is the pET-30a (+) vector with the P450cam gene cloned in.

Figure A.5 lists the nucleotide sequence of plasmid pET-30a $(+)$ P450cam containing vector pET-30a(+) and wild-type cytochrome P450cam gene cloned into NdeI (5'-end, nucleotide position 1596 in the pET-30a (+) vector sequence) and HindIII (3'-end, nucleotide position 173 in the pET-30a $(+)$ vector sequence). The numbering is correspondent to that of Figure A.5. 
Figure A.1 Amino acid sequence of full-length of WT cytochrome P450cam.

1 MTTETIQSNA NLAPLPPHVP EHLVFDFDMY NPSNLSAGVQ EAWAVLQESN 51 VPDLVWTRCN GGHWIATRGQ LIREAYEDYR HFSSECPFIP REAGEAYDFI 101 PTSMDPPEQR QFRALANQVV GMPVVDKLEN RIQELACSLI ESLRPQGQCN 151 FTEDYAEPFP IRIFMLLAGL PEEDIPHLKY LTDQMTRPDG SMTFAEAKEA 201 LYDYLIPIIE QRRQKPGTDA ISIVANGQVN GRPITSDEAK RMCGLLLVGG 251 LDTVVNFLSF SMEFLAKSPE HRQELIERPE RIPAACEELL RRFSLVADGR 301 ILTSDYEFHG VQLKKGDQIL LPQMLSGLDE RENACPMHVD FSRQKVSHTT 351 FGHGSHLCLG QHLARREIIV TLKEWLTRIP DFSIAPGAQI QHKSGIVSGV 401 QALPLVWDPA TTKAV 
Figure A.2 Nucleotide sequence of full-length WT cytochrome P450cam from Pseudomonas putida (5 bp upstream of PstI site, total 1578bp).

1 CTGCAGGATC GTTATCCGCT GGCCGATCTG ATCACCCAGC GTTTTTCCAT 51 CGACGAGGCC AGCAAGGCAC TTGAACTGGT CAAGGCAGGA GCACTGATCA 101 AACCCGTGAT CGACTCCACT CTTTAGCCAA CCCGCGTTCC AGGAGAACAA 151 CAACAATGAC GACTGAAACC ATACAAAGCA ACGCCAATCT TGCCCCTCTG 201 CCACCCCATG TGCCAGAGCA CCTGGTATTC GACTTCGACA TGTACAATCC 251 GTCGAATCTG TCTGCCGGCG TGCAGGAGGC CTGGGCAGTT CTGCAAGAAT 301 CAAACGTACC GGATCTGGTG TGGACTCGCT GCAACGGCGG ACACTGGATC 351 GCCACTCGCG GCCAACTGAT CCGTGAGGCC TATGAAGATT ACCGCCACTT 401 TTCCAGCGAG TGCCCGTTCA TCCCTCGTGA AGCCGGCGAA GCCTACGACT 451 TCATTCCCAC CTCGATGGAT CCGCCCGAGC AGCGCCAGTT TCGTGCGCTG 501 GCCAACCAAG TGGTTGGCAT GCCGGTGGTG GATAAGCTGG AGAACCGGAT 551 CCAGGAGCTG GCCTGCTCGC TGATCGAGAG CCTGCGCCCG CAAGGACAGT 601 GCAACTTCAC CGAGGACTAC GCCGAACCCT TCCCGATACG CATCTTCATG 651 CTGCTCGCAG GTCTACCGGA AGAAGATATC CCGCACTTGA AATACCTAAC 701 GGATCAGATG ACCCGTCCGG ATGGCAGCAT GACCTTCGCA GAGGCCAAGG 751 AGGCGCTCTA CGACTATCTG ATACCGATCA TCGAGCAACG CAGGCAGAAG 801 CCGGGAACCG ACGCTATCAG CATCGTTGCC AACGGCCAGG TCAATGGGCG 851 ACCGATCACC AGTGACGAAG CCAAGAGGAT GTGTGGCCTG TTACTGGTCG 901 GCGGCCTGGA TACGGTGGTC AATTTCCTCA GCTTCAGCAT GGAGTTCCTG 951 GCCAAAAGCC CGGAGCATCG CCAGGAGCTG ATCGAGCGTC CCGAGCGTAT 1001 TCCAGCCGCT TGCGAGGAAC TACTCCGGCG CTTCTCGCTG GTTGCCGATG 1051 GCCGCATCCT CACCTCCGAT TACGAGTTTC ATGGCGTGCA ACTGAAGAAA 1101 GGTGACCAGA TCCTGCTACC GCAGATGCTG TCTGGCCTGG ATGAGCGCGA 1151 AAACGCCTGC CCGATGCACG TCGACTTCAG TCGCCAAAAG GTTTCACACA 1201 CCACCTTTGG CCACGGCAGC CATCTGTGCC TTGGCCAGCA CCTGGCCCGC 1251 CGGGAAATCA TCGTCACCCT CAAGGAATGG CTGACCAGGA TTCCTGACTT 1301 CTCCATTGCC CCGGGTGCCC AGATTCAGCA CAAGAGCGGC ATCGTCAGCG 1351 GCGTGCAGGC ACTCCCTCTG GTCTGGGATC CGGCGACTAC CAAAGCGGTA 1401 TAAACACATG GGAGTGCGTG CTAAGTGAAC GCAAACGACA ACGTGGTCAT 1451 CGTCGGTACC GGACTGGCTG GCGTTGAGGT CGCCTTCGGC CTGCGCGCCA 1501 GCGGCTGGGA AGGCAATATC CGGTTGGTGG GGGATGCGAC GGTAATTCCC 1551 CATCACCTAC CACCGCTATC CAAAGCTT 
Figure A.3 Nucleotide sequence of vector pET-30a (+).

1 ATCCGGATAT AGTTCCTCCT TTCAGCAAAA AACCCCTCAA GACCCGTTTA 51 GAGGCCCCAA GGGGTTATGC TAGTTATTGC TCAGCGGTGG CAGCAGCCAA 101 CTCAGCTTCC TTTCGGGCTT TGTTAGCAGC CGGATCTCAG TGGTGGTGGT 151 GGTGGTGCTC GAGTGCGGCC GCAAGCTTGT CGACGGAGCT CGAATTCGGA 201 TCCGATATCA GCCATGGCCT TGTCGTCGTC GTCGGTACCC AGATCTGGGC 251 TGTCCATGTG CTGGCGTTCG AATTTAGCAG CAGCGGTTTC TTTCATACCA 301 GAACCGCGTG GCACCAGACC AGAAGAATGA TGATGATGAT GGTGCATATG 351 TATATCTCCT TCTTAAAGTT AAACAAAATT ATTTCTAGAG GGGAATTGTT 401 ATCCGCTCAC AATTCCCCTA TAGTGAGTCG TATTAATTTC GCGGGATCGA 451 GATCGATCTC GATCCTCTAC GCCGGACGCA TCGTGGCCGG CATCACCGGC 501 GCCACAGGTG CGGTTGCTGG CGCCTATATC GCCGACATCA CCGATGGGGA 551 AGATCGGGCT CGCCACTTCG GGCTCATGAG CGCTTGTTTC GGCGTGGGTA 601 TGGTGGCAGG CCCCGTGGCC GGGGGACTGT TGGGCGCCAT CTCCTTGCAT 651 GCACCATTCC TTGCGGCGGC GGTGCTCAAC GGCCTCAACC TACTACTGGG 701 CTGCTTCCTA ATGCAGGAGT CGCATAAGGG AGAGCGTCGA GATCCCGGAC 751 ACCATCGAAT GGCGCAAAAC CTTTCGCGGT ATGGCATGAT AGCGCCCGGA 801 AGAGAGTCAA TTCAGGGTGG TGAATGTGAA ACCAGTAACG TTATACGATG 851 TCGCAGAGTA TGCCGGTGTC TCTTATCAGA CCGTTTCCCG CGTGGTGAAC 901 CAGGCCAGCC ACGTTTCTGC GAAAACGCGG GAAAAAGTGG AAGCGGCGAT 951 GGCGGAGCTG AATTACATTC CCAACCGCGT GGCACAACAA CTGGCGGGCA 1001 AACAGTCGTT GCTGATTGGC GTTGCCACCT CCAGTCTGGC CCTGCACGCG 1051 CCGTCGCAAA TTGTCGCGGC GATTAAATCT CGCGCCGATC AACTGGGTGC 1101 CAGCGTGGTG GTGTCGATGG TAGAACGAAG CGGCGTCGAA GCCTGTAAAG 1151 CGGCGGTGCA CAATCTTCTC GCGCAACGCG TCAGTGGGCT GATCATTAAC 1201 TATCCGCTGG ATGACCAGGA TGCCATTGCT GTGGAAGCTG CCTGCACTAA 1251 TGTTCCGGCG TTATTTCTTG ATGTCTCTGA CCAGACACCC ATCAACAGTA 1301 TTATTTTCTC CCATGAAGAC GGTACGCGAC TGGGCGTGGA GCATCTGGTC 1351 GCATTGGGTC ACCAGCAAAT CGCGCTGTTA GCGGGCCCAT TAAGTTCTGT 1401 CTCGGCGCGT CTGCGTCTGG CTGGCTGGCA TAAATATCTC ACTCGCAATC 1451 AAATTCAGCC GATAGCGGAA CGGGAAGGCG ACTGGAGTGC CATGTCCGGT 1501 TTTCAACAAA CCATGCAAAT GCTGAATGAG GGCATCGTTC CCACTGCGAT 1551 GCTGGTTGCC AACGATCAGA TGGCGCTGGG CGCAATGCGC GCCATTACCG 1601 AGTCCGGGCT GCGCGTTGGT GCGGACATCT CGGTAGTGGG ATACGACGAT 1651 ACCGAAGACA GCTCATGTTA TATCCCGCCG TTAACCACCA TCAAACAGGA 1701 TTTTCGCCTG CTGGGGCAAA CCAGCGTGGA CCGCTTGCTG CAACTCTCTC 1751 AGGGCCAGGC GGTGAAGGGC AATCAGCTGT TGCCCGTCTC ACTGGTGAAA 1801 AGAAAAACCA CCCTGGCGCC CAATACGCAA ACCGCCTCTC CCCGCGCGTT 1851 GGCCGATTCA TTAATGCAGC TGGCACGACA GGTTTCCCGA CTGGAAAGCG 
1901 GGCAGTGAGC GCAACGCAAT TAATGTAAGT TAGCTCACTC ATTAGGCACC 1951 GGGATCTCGA CCGATGCCCT TGAGAGCCTT CAACCCAGTC AGCTCCTTCC 2001 GGTGGGCGCG GGGCATGACT ATCGTCGCCG CACTTATGAC TGTCTTCTTT 2051 ATCATGCAAC TCGTAGGACA GGTGCCGGCA GCGCTCTGGG TCATTTTCGG 2101 CGAGGACCGC TTTCGCTGGA GCGCGACGAT GATCGGCCTG TCGCTTGCGG 2151 TATTCGGAAT CTTGCACGCC CTCGCTCAAG CCTTCGTCAC TGGTCCCGCC 2201 ACCAAACGTT TCGGCGAGAA GCAGGCCATT ATCGCCGGCA TGGCGGCCCC 2251 ACGGGTGCGC ATGATCGTGC TCCTGTCGTT GAGGACCCGG CTAGGCTGGC 2301 GGGGTTGCCT TACTGGTTAG CAGAATGAAT CACCGATACG CGAGCGAACG 2351 TGAAGCGACT GCTGCTGCAA AACGTCTGCG ACCTGAGCAA CAACATGAAT 2401 GGTCTTCGGT TTCCGTGTTT CGTAAAGTCT GGAAACGCGG AAGTCAGCGC 2451 CCTGCACCAT TATGTTCCGG ATCTGCATCG CAGGATGCTG CTGGCTACCC 2501 TGTGGAACAC CTACATCTGT ATTAACGAAG CGCTGGCATT GACCCTGAGT 2551 GATTTTTCTC TGGTCCCGCC GCATCCATAC CGCCAGTTGT TTACCCTCAC 2601 AACGTTCCAG TAACCGGGCA TGTTCATCAT CAGTAACCCG TATCGTGAGC 2651 ATCCTCTCTC GTTTCATCGG TATCATTACC CCCATGAACA GAAATCCCCC 2701 TTACACGGAG GCATCAGTGA CCAAACAGGA AAAAACCGCC CTTAACATGG 2751 CCCGCTTTAT CAGAAGCCAG ACATTAACGC TTCTGGAGAA ACTCAACGAG 2801 CTGGACGCGG ATGAACAGGC AGACATCTGT GAATCGCTTC ACGACCACGC 2851 TGATGAGCTT TACCGCAGCT GCCTCGCGCG TTTCGGTGAT GACGGTGAAA 2901 ACCTCTGACA CATGCAGCTC CCGGAGACGG TCACAGCTTG TCTGTAAGCG 2951 GATGCCGGGA GCAGACAAGC CCGTCAGGGC GCGTCAGCGG GTGTTGGCGG 3001 GTGTCGGGGC GCAGCCATGA CCCAGTCACG TAGCGATAGC GGAGTGTATA 3051 CTGGCTTAAC TATGCGGCAT CAGAGCAGAT TGTACTGAGA GTGCACCATA 3101 TATGCGGTGT GAAATACCGC ACAGATGCGT AAGGAGAAAA TACCGCATCA 3151 GGCGCTCTTC CGCTTCCTCG CTCACTGACT CGCTGCGCTC GGTCGTTCGG 3201 CTGCGGCGAG CGGTATCAGC TCACTCAAAG GCGGTAATAC GGTTATCCAC 3251 AGAATCAGGG GATAACGCAG GAAAGAACAT GTGAGCAAAA GGCCAGCAAA 3301 AGGCCAGGAA CCGTAAAAAG GCCGCGTTGC TGGCGTTTTT CCATAGGCTC 3351 CGCCCCCCTG ACGAGCATCA CAAAAATCGA CGCTCAAGTC AGAGGTGGCG 3401 AAACCCGACA GGACTATAAA GATACCAGGC GTTTCCCCCT GGAAGCTCCC 3451 TCGTGCGCTC TCCTGTTCCG ACCCTGCCGC TTACCGGATA CCTGTCCGCC 3501 TTTCTCCCTT CGGGAAGCGT GGCGCTTTCT CATAGCTCAC GCTGTAGGTA 3551 TCTCAGTTCG GTGTAGGTCG TTCGCTCCAA GCTGGGCTGT GTGCACGAAC 3601 CCCCCGTTCA GCCCGACCGC TGCGCCTTAT CCGGTAACTA TCGTCTTGAG 3651 TCCAACCCGG TAAGACACGA CTTATCGCCA CTGGCAGCAG CCACTGGTAA 3701 CAGGATTAGC AGAGCGAGGT ATGTAGGCGG TGCTACAGAG TTCTTGAAGT 3751 GGTGGCCTAA CTACGGCTAC ACTAGAAGGA CAGTATTTGG TATCTGCGCT 3801 CTGCTGAAGC CAGTTACCTT CGGAAAAAGA GTTGGTAGCT CTTGATCCGG 3851 CAAACAAACC ACCGCTGGTA GCGGTGGTTT TTTTGTTTGC AAGCAGCAGA 
3901 TTACGCGCAG AAAAAAAGGA TCTCAAGAAG ATCCTTTGAT CTTTTCTACG 3951 GGGTCTGACG CTCAGTGGAA CGAAAACTCA CGTTAAGGGA TTTTGGTCAT 4001 GAACAATAAA ACTGTCTGCT TACATAAACA GTAATACAAG GGGTGTTATG 4051 AGCCATATTC AACGGGAAAC GTCTTGCTCT AGGCCGCGAT TAAATTCCAA 4101 CATGGATGCT GATTTATATG GGTATAAATG GGCTCGCGAT AATGTCGGGC 4151 AATCAGGTGC GACAATCTAT CGATTGTATG GGAAGCCCGA TGCGCCAGAG 4201 TTGTTTCTGA AACATGGCAA AGGTAGCGTT GCCAATGATG TTACAGATGA 4251 GATGGTCAGA CTAAACTGGC TGACGGAATT TATGCCTCTT CCGACCATCA 4301 AGCATTTTAT CCGTACTCCT GATGATGCAT GGTTACTCAC CACTGCGATC 4351 CCCGGGAAAA CAGCATTCCA GGTATTAGAA GAATATCCTG ATTCAGGTGA 4401 AAATATTGTT GATGCGCTGG CAGTGTTCCT GCGCCGGTTG CATTCGATTC 4451 CTGTTTGTAA TTGTCCTTTT AACAGCGATC GCGTATTTCG TCTCGCTCAG 4501 GCGCAATCAC GAATGAATAA CGGTTTGGTT GATGCGAGTG ATTTTGATGA 4551 CGAGCGTAAT GGCTGGCCTG TTGAACAAGT CTGGAAAGAA ATGCATAAAC 4601 TTTTGCCATT CTCACCGGAT TCAGTCGTCA CTCATGGTGA TTTCTCACTT 4651 GATAACCTTA TTTTTGACGA GGGGAAATTA ATAGGTTGTA TTGATGTTGG 4701 ACGAGTCGGA ATCGCAGACC GATACCAGGA TCTTGCCATC CTATGGAACT 4751 GCCTCGGTGA GTTTTCTCCT TCATTACAGA AACGGCTTTT TCAAAAATAT 4801 GGTATTGATA ATCCTGATAT GAATAAATTG CAGTTTCATT TGATGCTCGA 4851 TGAGTTTTTC TAAGAATTAA TTCATGAGCG GATACATATT TGAATGTATT 4901 TAGAAAAATA AACAAATAGG GGTTCCGCGC ACATTTCCCC GAAAAGTGCC 4951 ACCTGAAATT GTAAACGTTA ATATTTTGTT AAAATTCGCG TTAAATTTTT 5001 GTTAAATCAG CTCATTTTTT AACCAATAGG CCGAAATCGG CAAAATCCCT 5051 TATAAATCAA AAGAATAGAC CGAGATAGGG TTGAGTGTTG TTCCAGTTTG 5101 GAACAAGAGT CCACTATTAA AGAACGTGGA CTCCAACGTC AAAGGGCGAA 5151 AAACCGTCTA TCAGGGCGAT GGCCCACTAC GTGAACCATC ACCCTAATCA 5201 AGTTTTTTGG GGTCGAGGTG CCGTAAAGCA CTAAATCGGA ACCCTAAAGG 5251 GAGCCCCCGA TTTAGAGCTT GACGGGGAAA GCCGGCGAAC GTGGCGAGAA 5301 AGGAAGGGAA GAAAGCGAAA GGAGCGGGCG CTAGGGCGCT GCAAGTGTA 5351 GCGGTCACGC TGCGCGTAAC CACCACACCC GCCGCGCTTA ATGCGCCGCT 5401 ACAGGGCGCG TCCCATTCGC CA 


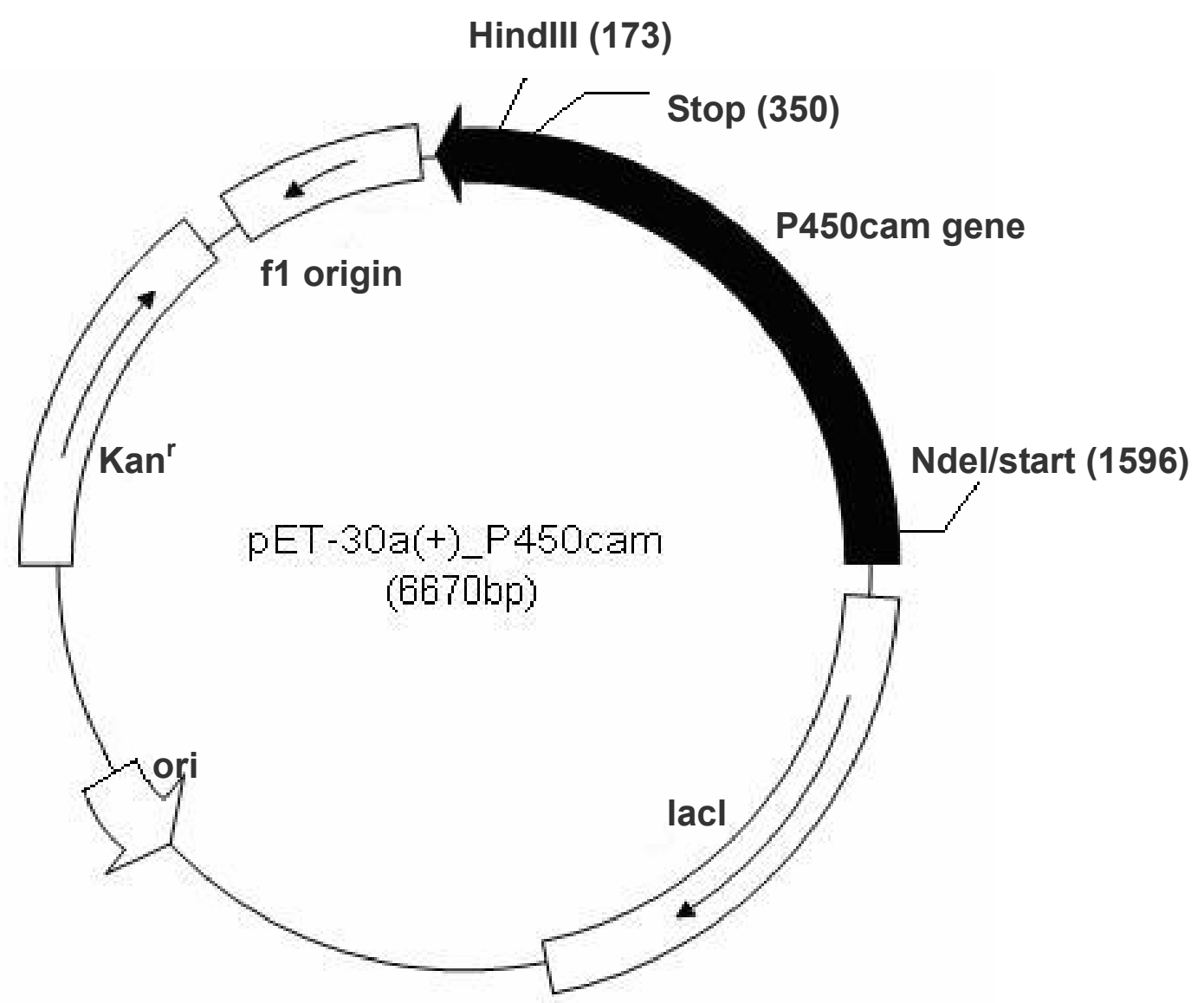

Figure A.4 Plasmid map of pET-30a (+)_P450cam. 
Figure A.5 Nucleotide sequence of plasmid containing vector pET-30a(+) and WT cytochrome P450cam gene. $(152+1426+5072=6670 \mathrm{bp})$

1 ATCCGGATAT AGTTCCTCCT TTCAGCAAAA AACCCCTCAA GACCCGTTTA 51 GAGGCCCCAA GGGGTTATGC TAGTTATTGC TCAGCGGTGG CAGCAGCCAA 101 CTCAGCTTCC TTTCGGGCTT TGTTAGCAGC CGGATCTCAG TGGTGGTGGT 151 GGTGGTGCTC GAGTGCGGCC GCAAGCTTTG GATAGCGGTG GTAGGTGATG 201 GGGAATTACC GTCGCATCCC CCACCAACCG GATATTGCCT TCCCAGCCGC 251 TGGCGCGCAG GCCGAAGGCG ACCTCAACGC CAGCCAGTCC GGTACCGACG 301 ATGACCACGT TGTCGTTTGC GTTCACTTAG CACGCACTCC CATGTGTTTA 351 TACCGCTTTG GTAGTCGCCG GATCCCAGAC CAGAGGGAGT GCCTGCACGC 401 CGCTGACGAT GCCGCTCTTG TGCTGAATCT GGGCACCCGG GGCAATGGAG 451 AAGTCAGGAA TCCTGGTCAG CCATTCCTTG AGGGTGACGA TGATTTCCCG 501 GCGGGCCAGG TGCTGGCCAA GGCACAGATG GCTGCCGTGG CCAAAGGTGG 551 TGTGTGAAAC CTTTTGGCGA CTGAAGTCGA CGTGCATCGG GCAGGCGTTT 601 TCGCGCTCAT CCAGGCCAGA CAGCATCTGC GGTAGCAGGA TCTGGTCACC 651 TTTCTTCAGT TGCACGCCAT GAAACTCGTA ATCGGAGGTG AGGATGCGGC 701 CATCGGCAAC CAGCGAGAAG CGCCGGAGTA GTTCCTCGCA AGCGGCTGGA 751 ATACGCTCGG GACGCTCGAT CAGCTCCTGG CGATGCTCCG GGCTTTTGGC 801 CAGGAACTCC ATGCTGAAGC TGAGGAAATT GACCACCGTA TCCAGGCCGC 851 CGACCAGTAA CAGGCCACAC ATCCTCTTGG CTTCGTCACT GGTGATCGGT 901 CGCCCATTGA CCTGGCCGTT GGCAACGATG CTGATAGCGT CGGTTCCCGG 951 CTTCTGCCTG CGTTGCTCGA TGATCGGTAT CAGATAGTC GTAGAGCGCCT 1001 CCTTGGCCTC TGCGAAGGTC ATGCTGCCAT CCGGACGGGT CATCTGATCC 1051 GTTAGGTATT TCAAGTGCGG GATATCTTCT TCCGGTAGAC CTGCGAGCAG 1101 CATGAAGATG CGTATCGGGA AGGGTTCGGC GTAGTCCTCG GTGAAGTTGC 1151 ACTGTCCTTG CGGGCGCAGG CTCTCGATCA GCGAGCAGGC CAGCTCCTGG 1201 ATCCGGTTCT CCAGCTTATC CACCACCGGC ATGCCAACCA CTTGGTTGGC 1251 CAGCGCACGA AACTGGCGCT GCTCGGGCGG ATCCATCGAG GTGGGAATGA 1301 AGTCGTAGGC TTCGCCGGCT TCACGAGGGA TGAACGGGCA CTCGCTGGAA 1351 AAGTGGCGGT AATCTTCATA GGCCTCACGG ATCAGTTGGC CGCGAGTGGC 1401 GATCCAGTGT CCGCCGTTGC AGCGAGTCCA CACCAGATCC GGTACGTTTG 1451 ATTCTTGCAG AACTGCCCAG GCCTCCTGCA CGCCGGCAGA CAGATTCGAC 1501 GGATTGTACA TGTCGAAGTC GAATACCAGG TGCTCTGGCA CATGGGGTGG 1551 CAGAGGGGCA AGATTGGCGT TGCTTTGTAT GGTTTCAGTC GTCATATGTA 1601 TATCTCCTTC TTAAAGTTAA ACAAAATTAT TTCTAGAGGG GAATTGTTAT 1651 CCGCTCACAA TTCCCCTATA GTGAGTCGTA TTAATTTCGC GGGATCGAGA 1701 TCGATCTCGA TCCTCTACGC CGGACGCATC GTGGCCGGCA TCACCGGCGC 1751 CACAGGTGCG GTTGCTGGCG CCTATATCGC CGACATCACC GATGGGGAAG 1801 ATCGGGCTCG CCACTTCGGG CTCATGAGCG CTTGTTTCGG CGTGGGTATG 
1851 GTGGCAGGCC CCGTGGCCGG GGGACTGTTG GGCGCCATCT CCTTGCATGC 1901 ACCATTCCTT GCGGCGGCGG TGCTCAACGG CCTCAACCTA CTACTGGGCT 1951 GCTTCCTAAT GCAGGAGTCG CATAAGGGAG AGCGTCGAGA TCCCGGACAC 2001 CATCGAATGG CGCAAAACCT TTCGCGGTAT GGCATGATAG CGCCCGGAAG 2051 AGAGTCAATT CAGGGTGGTG AATGTGAAAC CAGTAACGTT ATACGATGTC 2101 GCAGAGTATG CCGGTGTCTC TTATCAGACC GTTTCCCGCG TGGTGAACCA 2151 GGCCAGCCAC GTTTCTGCGA AAACGCGGGA AAAAGTGGAA GCGGCGATGG 2201 CGGAGCTGAA TTACATTCCC AACCGCGTGG CACAACAACT GGCGGGCAAA 2251 CAGTCGTTGC TGATTGGCGT TGCCACCTCC AGTCTGGCCC TGCACGCGCC 2301 GTCGCAAATT GTCGCGGCGA TTAAATCTCG CGCCGATCAA CTGGGTGCCA 2351 GCGTGGTGGT GTCGATGGTA GAACGAAGCG GCGTCGAAGC CTGTAAAGCG 2401 GCGGTGCACA ATCTTCTCGC GCAACGCGTC AGTGGGCTGA TCATTAACTA 2451 TCCGCTGGAT GACCAGGATG CCATTGCTGT GGAAGCTGCC TGCACTAATG 2501 TTCCGGCGTT ATTTCTTGAT GTCTCTGACC AGACACCCAT CAACAGTATT 2551 ATTTTCTCCC ATGAAGACGG TACGCGACTG GGCGTGGAGC ATCTGGTCGC 2601 ATTGGGTCAC CAGCAAATCG CGCTGTTAGC GGGCCCATTA AGTTCTGTCT 2651 CGGCGCGTCT GCGTCTGGCT GGCTGGCATA AATATCTCAC TCGCAATCAA 2701 ATTCAGCCGA TAGCGGAACG GGAAGGCGAC TGGAGTGCCA TGTCCGGTTT 2751 TCAACAAACC ATGCAAATGC TGAATGAGGG CATCGTTCCC ACTGCGATGC 2801 TGGTTGCCAA CGATCAGATG GCGCTGGGCG CAATGCGCGC CATTACCGAG 2851 TCCGGGCTGC GCGTTGGTGC GGACATCTCG GTAGTGGGAT ACGACGATAC 2901 CGAAGACAGC TCATGTTATA TCCCGCCGTT AACCACCATC AAACAGGATT 2951 TTCGCCTGCT GGGGCAAACC AGCGTGGACC GCTTGCTGCA ACTCTCTCAG 3001 GGCCAGGCGG TGAAGGGCAA TCAGCTGTTG CCCGTCTCAC TGGTGAAAAG 3051 AAAAACCACC CTGGCGCCCA ATACGCAAAC CGCCTCTCCC CGCGCGTTGG 3101 CCGATTCATT AATGCAGCTG GCACGACAGG TTTCCCGACT GGAAAGCGGG 3151 CAGTGAGCGC AACGCAATTA ATGTAAGTTA GCTCACTCAT TAGGCACCGG 3201 GATCTCGACC GATGCCCTTG AGAGCCTTCA ACCCAGTCAG CTCCTTCCGG 3251 TGGGCGCGGG GCATGACTAT CGTCGCCGCA CTTATGACTG TCTTCTTTAT 3301 CATGCAACTC GTAGGACAGG TGCCGGCAGC GCTCTGGGTC ATTTTCGGCG 3351 AGGACCGCTT TCGCTGGAGC GCGACGATGA TCGGCCTGTC GCTTGCGGTA 3401 TTCGGAATCT TGCACGCCCT CGCTCAAGCC TTCGTCACTG GTCCCGCCAC 3451 CAAACGTTTC GGCGAGAAGC AGGCCATTAT CGCCGGCATG GCGGCCCCAC 3501 GGGTGCGCAT GATCGTGCTC CTGTCGTTGA GGACCCGGCT AGGCTGGCGG 3551 GGTTGCCTTA CTGGTTAGCA GAATGAATCA CCGATACGCG AGCGAACGTG 3601 AAGCGACTGC TGCTGCAAAA CGTCTGCGAC CTGAGCAACA ACATGAATGG 3651 TCTTCGGTTT CCGTGTTTCG TAAAGTCTGG AAACGCGGAA GTCAGCGCCC 3701 TGCACCATTA TGTTCCGGAT CTGCATCGCA GGATGCTGCT GGCTACCCTG 3751 TGGAACACCT ACATCTGTAT TAACGAAGCG CTGGCATTGA CCCTGAGTGA 3801 TTTTTCTCTG GTCCCGCCGC ATCCATACCG CCAGTTGTTT ACCCTCACAA 
3851 CGTTCCAGTA ACCGGGCATG TTCATCATCA GTAACCCGTA TCGTGAGCAT 3901 CCTCTCTCGT TTCATCGGTA TCATTACCCC CATGAACAGA AATCCCCCTT 3951 ACACGGAGGC ATCAGTGACC AAACAGGAAA AAACCGCCCT TAACATGGCC 4001 CGCTTTATCA GAAGCCAGAC ATTAACGCTT CTGGAGAAAC TCAACGAGCT 4051 GGACGCGGAT GAACAGGCAG ACATCTGTGA ATCGCTTCAC GACCACGCTG 4101 ATGAGCTTTA CCGCAGCTGC CTCGCGCGTT TCGGTGATGA CGGTGAAAAC 4151 CTCTGACACA TGCAGCTCCC GGAGACGGTC ACAGCTTGTC TGTAAGCGGA 4201 TGCCGGGAGC AGACAAGCCC GTCAGGGCGC GTCAGCGGGT GTTGGCGGGT 4251 GTCGGGGCGC AGCCATGACC CAGTCACGTA GCGATAGCGG AGTGTATACT 4301 GGCTTAACTA TGCGGCATCA GAGCAGATTG TACTGAGAGT GCACCATATA 4351 TGCGGTGTGA AATACCGCAC AGATGCGTAA GGAGAAAATA CCGCATCAGG 4401 CGCTCTTCCG CTTCCTCGCT CACTGACTCG CTGCGCTCGG TCGTTCGGCT 4451 GCGGCGAGCG GTATCAGCTC ACTCAAAGGC GGTAATACGG TTATCCACAG 4501 AATCAGGGGA TAACGCAGGA AAGAACATGT GAGCAAAAGG CCAGCAAAAG 4551 GCCAGGAACC GTAAAAAGGC CGCGTTGCTG GCGTTTTTCC ATAGGCTCCG 4601 CCCCCCTGAC GAGCATCACA AAAATCGACG CTCAAGTCAG AGGTGGCGAA 4651 ACCCGACAGG ACTATAAAGA TACCAGGCGT TTCCCCCTGG AAGCTCCCTC 4701 GTGCGCTCTC CTGTTCCGAC CCTGCCGCTT ACCGGATACC TGTCCGCCTT 4751 TCTCCCTTCG GGAAGCGTGG CGCTTTCTCA TAGCTCACGC TGTAGGTATC 4801 TCAGTTCGGT GTAGGTCGTT CGCTCCAAGC TGGGCTGTGT GCACGAACCC 4851 CCCGTTCAGC CCGACCGCTG CGCCTTATCC GGTAACTATC GTCTTGAGTC 4901 CAACCCGGTA AGACACGACT TATCGCCACT GGCAGCAGCC ACTGGTAACA 4951 GGATTAGCAG AGCGAGGTAT GTAGGCGGTG CTACAGAGTT CTTGAAGTGG 5001 TGGCCTAACT ACGGCTACAC TAGAAGGACA GTATTTGGTA TCTGCGCTCT 5051 GCTGAAGCCA GTTACCTTCG GAAAAAGAGT TGGTAGCTCT TGATCCGGCA 5101 AACAAACCAC CGCTGGTAGC GGTGGTTTTT TTGTTTGCAA GCAGCAGATT 5151 ACGCGCAGAA AAAAAGGATC TCAAGAAGAT CCTTTGATCT TTTCTACGGG 5201 GTCTGACGCT CAGTGGAACG AAAACTCACG TTAAGGGATT TTGGTCATGA 5251 ACAATAAAAC TGTCTGCTTA CATAAACAGT AATACAAGGG GTGTTATGAG 5301 CCATATTCAA CGGGAAACGT CTTGCTCTAG GCCGCGATTA AATTCCAACA 5351 TGGATGCTGA TTTATATGGG TATAAATGGG CTCGCGATAA TGTCGGGCAA 5401 TCAGGTGCGA CAATCTATCG ATTGTATGGG AAGCCCGATG CGCCAGAGTT 5451 GTTTCTGAAA CATGGCAAAG GTAGCGTTGC CAATGATGTT ACAGATGAGA 5501 TGGTCAGACT AAACTGGCTG ACGGAATTTA TGCCTCTTCC GACCATCAAG 5551 CATTTTATCC GTACTCCTGA TGATGCATGG TTACTCACCA CTGCGATCCC 5601 CGGGAAAACA GCATTCCAGG TATTAGAAGA ATATCCTGAT TCAGGTGAAA 5651 ATATTGTTGA TGCGCTGGCA GTGTTCCTGC GCCGGTTGCA TTCGATTCCT 5701 GTTTGTAATT GTCCTTTTAA CAGCGATCGC GTATTTCGTC TCGCTCAGGC 5751 GCAATCACGA ATGAATAACG GTTTGGTTGA TGCGAGTGAT TTTGATGACG 5801 AGCGTAATGG CTGGCCTGTT GAACAAGTCT GGAAAGAAAT GCATAAACTT 
5851 TTGCCATTCT CACCGGATTC AGTCGTCACT CATGGTGATT TCTCACTTGA 5901 TAACCTTATT TTTGACGAGG GGAAATTAAT AGGTTGTATT GATGTTGGAC 5951 GAGTCGGAAT CGCAGACCGA TACCAGGATC TTGCCATCCT ATGGAACTGC 6001 CTCGGTGAGT TTTCTCCTTC ATTACAGAAA CGGCTTTTTC AAAAATATGG 6051 TATTGATAAT CCTGATATGA ATAAATTGCA GTTTCATTTG ATGCTCGATG 6101 AGTTTTTCTA AGAATTAATT CATGAGCGGA TACATATTTG AATGTATTTA 6151 GAAAAATAAA CAAATAGGGG TTCCGCGCAC ATTTCCCCGA AAAGTGCCAC 6201 CTGAAATTGT AAACGTTAAT ATTTTGTTAA AATTCGCGTT AAATTTTTGT 6251 TAAATCAGCT CATTTTTTAA CCAATAGGCC GAAATCGGCA AAATCCCTTA 6301 TAAATCAAAA GAATAGACCG AGATAGGGTT GAGTGTTGTT CCAGTTTGGA 6351 ACAAGAGTCC ACTATTAAAG AACGTGGACT CCAACGTCAA AGGGCGAAAA 6401 ACCGTCTATC AGGGCGATGG CCCACTACGT GAACCATCAC CCTAATCAAG 6451 TTTTTTGGGG TCGAGGTGCC GTAAAGCACT AAATCGGAAC CCTAAAGGGA 6501 GCCCCCGATT TAGAGCTTGA CGGGGAAAGC CGGCGAACGT GGCGAGAAAG 6551 GAAGGGAAGA AAGCGAAAGG AGCGGGCGCT AGGGCGCTGG CAAGTGTAGC 6601 GGTCACGCTG CGCGTAACCA CCACACCCGC CGCGCTTAAT GCGCCGCTAC 6651 AGGGCGCGTC CCATTCGCCA 


\section{APPENDIX B}

\section{Sequences of Sperm Whale Myoglobin and pET-30a (+) Vector}

\section{General information}

The sperm whale myoglobin includes 154 amino acids.

Figure B.1 lists the amino acid sequence of full-length, wild-type sperm whale myoglobin.

Figure B.1 lists the nucleotide sequence of the synthesized sperm whale myoglobin gene as designed from the amio acid sequence including the ribosome binding site. (Springer, B. et al, Proc Natl Acad Sci USA, 1987, 84, 8961-8965)

Figure B.3 displays a map of plasmid pET-30a $(+)$ _Mb, which is the pET-30a $(+)$ vector with the sperm whale myoglobin gene cloned in.

Figure B.4 lists the nucleotide sequence of plasmid pET-30a $(+)$ _Mb containing vector pET-30a(+) and wild-type sperm whale myoglobin gene cloned into NdeI (5'-end, nucleotide position 713 in the pET-30a $(+)$ vector sequence) and KpNI (3'-end, nucleotide position 238 in the pET-30a $(+)$ vector sequence). The numbering is correspondent to that of Figure B.4. 
Figure B.1 Amino acid sequence of full-length of sperm whale myoglobin (154aa).

1 MVLSEGEWQL VLHVWAKVEA DVAGHGQDIL IRLFKSHPET LEKFDRFKHL 51 KTEAEMKASE DLKKHGVTVL TALGAILKKK GHHEAELKPL AQSHATKHKI 101 PIKYLEFISE AIIHVLHSRH PGNFGADAQG AMNKALELFR KDIAAKYKEL 151 GYQG

Figure B.2 Nucleotide sequence of full-length sperm whale myoglobin (507bp).

1 CTGCAGATAA CTAACTAAAG GAGAACAACA ACAATGGTTC TGTCTGAAGG 51 TGAATGGCAG CTGGTTCTGC ATGTTTGGGC TAAAGTTGAA GCTGACGTCG 101 CTGGTCATGG TCAGGACATC TTGATTCGAC TGTTCAAATC TCATCCGGAA 151 ACTCTGGAAA AATTCGATCG TTTCAAACAT CTGAAAACTG AAGCTGAAAT 201 GAAAGCTTCT GAAGATCTGA AAAAACATGG TGTTACCGTG TTAACTGCCC 251 TAGGTGCTAT CCTTAAGAAA AAAGGGCATC ATGAAGCTGA GCTCAAACCG 301 CTTGCGCAAT CGCATGCTAC TAAACATAAG ATCCCGATCA AATACCTGGA 351 ATTCATCTCT GAAGCGATCA TCCATGTTCT GCATTCTAGA CATCCAGGTA 401 ACTTCGGTGC TGACGCTCAG GGTGCTATGA ACAAAGCTCT CGAGCTGTTC 451 CGTAAAGATA TCGCTGCTAA GTACAAAGAA CTGGGTTACC AGGGTTAATG 501 AGGTACC 


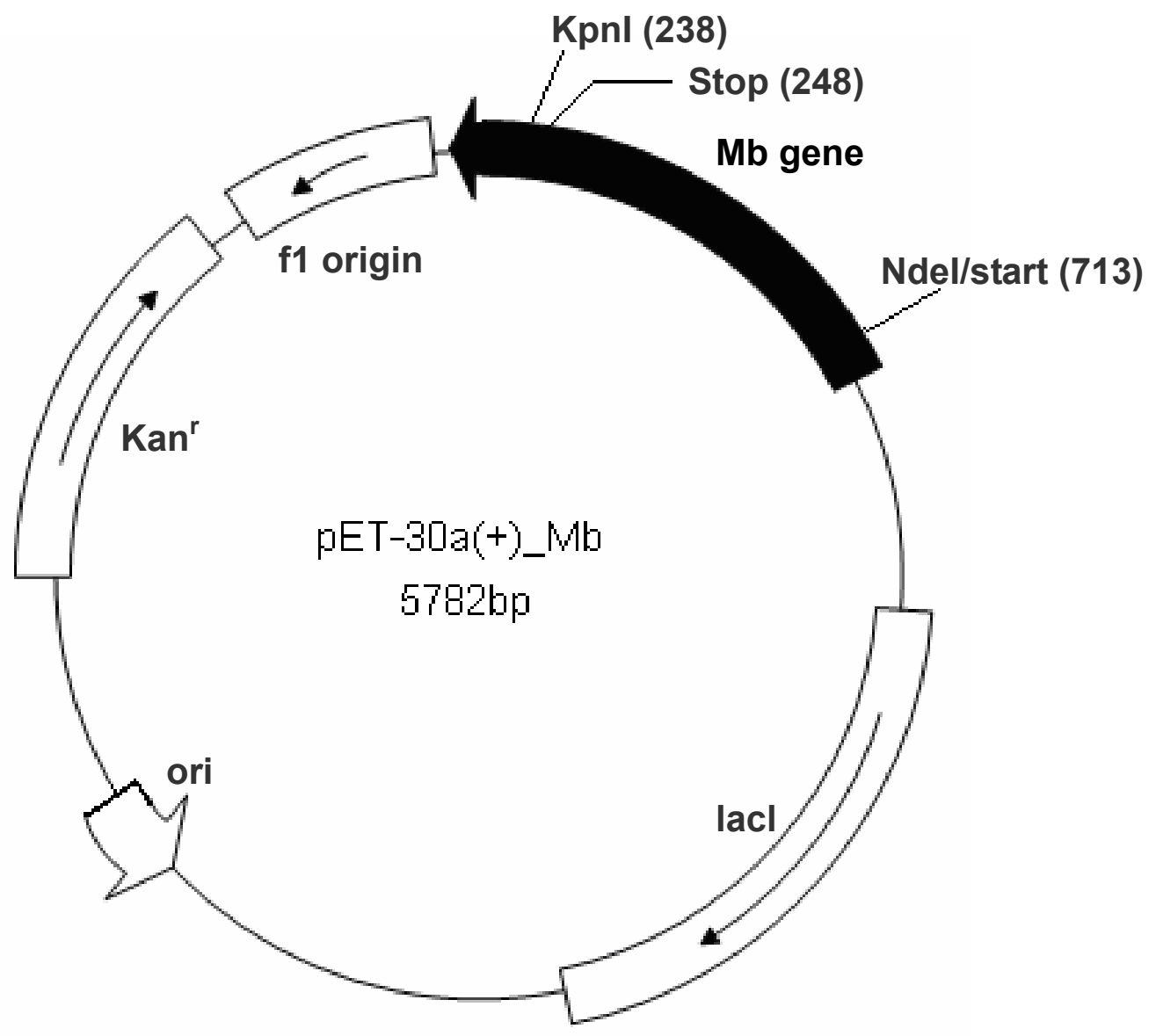

Figure B.3 Plasmid map of pET-30a (+)_Mb. 
Figure B.4 Nucleotide sequence of plasmid containing vector pET-30a (+) and sperm whale myglobin gene (5782bp).

1 ATCCGGATAT AGTTCCTCCT TTCAGCAAAA AACCCCTCAA GACCCGTTTA 51 GAGGCCCCAA GGGGTTATGC TAGTTATTGC TCAGCGGTGG CAGCAGCCAA 101 CTCAGCTTCC TTTCGGGCTT TGTTAGCAGC CGGATCTCAG TGGTGGTGGT 151 GGTGGTGCTC GAGTGCGGCC GCAAGCTTGT CGACGGAGCT CGAATTCGGA 201 TCCGATATCA GCCATGGCCT TGTCGTCGTC GTCGGTACCT CATTA ACCCT 251 GGTAACCCAG TTCTTTGTAC TTAGCAGCGA TATCTTTACG GAACAGCTCG 301 AGAGCTTTGT TCATAGCACC CTGAGCGTCA GCACCGAAGT CACCTGGATG 351 TCTAGAATGC AGAACATGGA TGATCGCTTC AGAGATGAAT TCCAGGTATT 401 TGATCGGGAT CTTATGTTTA GTAGCATGCG ATTGCGCAAG CGGTTTGAGC 451 TCAGCTTCAT GATGCCCTTT TTTCTTAAGG ATAGCACCTA GGGCAGTTAA 501 CACGGTAACA CCATGTTTTT TCAGATCTTC AGAAGCTTTC ATTTCAGCTT 551 CAGTTTTCAG ATGTTTGAAA CGATCGAATT TTTCCAGAGT TTCCGGATGA 601 GATTTGAACA GTCGAATCAA GATGTCCTGA CCATGACCAG CGACGTCAGC 651 TTCAACTTTA GCCCAAACAT GCAGAACCAG CTGCCATTCA CCTTCAGACA 701 GAACCATATG TATATCTCCT TCTTAAAGTT AAACAAAATT ATTTCTAGAG 751 GGGAATTGTT ATCCGCTCAC AATTCCCCTA TAGTGAGTCG TATTAATTTC 801 GCGGGATCGA GATCGATCTC GATCCTCTAC GCCGGACGCA TCGTGGCCGG 851 CATCACCGGC GCCACAGGTG CGGTTGCTGG CGCCTATATC GCCGACATCA 901 CCGATGGGGA AGATCGGGCT CGCCACTTCG GGCTCATGAG CGCTTGTTTC 951 GGCGTGGGTA TGGTGGCAGG CCCCGTGGCC GGGGGACTGT TGGGCGCCAT 1001 CTCCTTGCAT GCACCATTCC TTGCGGCGGC GGTGCTCAAC GGCCTCAACC 1051 TACTACTGGG CTGCTTCCTA ATGCAGGAGT CGCATAAGGG AGAGCGTCGA 1101 GATCCCGGAC ACCATCGAAT GGCGCAAAAC CTTTCGCGGT ATGGCATGAT 1151 AGCGCCCGGA AGAGAGTCAA TTCAGGGTGG TGAATGTGAA ACCAGTAACG 1201 TTATACGATG TCGCAGAGTA TGCCGGTGTC TCTTATCAGA CCGTTTCCCG 1251CGTGGTGAAC CAGGCCAGCC ACGTTTCTGC GAAAACGCGG GAAAAAGTGG 1301 AAGCGGCGAT GGCGGAGCTG AATTACATTC CCAACCGCGT GGCACAACAA 1351 CTGGCGGGCA AACAGTCGTT GCTGATTGGC GTTGCCACCT CCAGTCTGGC 1401 CCTGCACGCG CCGTCGCAAA TTGTCGCGGC GATTAAATCT CGCGCCGATC 1451 AACTGGGTGC CAGCGTGGTG GTGTCGATGG TAGAACGAAG CGGCGTCGAA 1501 GCCTGTAAAG CGGCGGTGCA CAATCTTCTC GCGCAACGCG TCAGTGGGCT 1551 GATCATTAAC TATCCGCTGG ATGACCAGGA TGCCATTGCT GTGGAAGCTG 1601 CCTGCACTAA TGTTCCGGCG TTATTTCTTG ATGTCTCTGA CCAGACACCC 1651 ATCAACAGTA TTATTTTCTC CCATGAAGAC GGTACGCGAC TGGGCGTGGA 1701 GCATCTGGTC GCATTGGGTC ACCAGCAAAT CGCGCTGTTA GCGGGCCCAT 1751 TAAGTTCTGT CTCGGCGCGT CTGCGTCTGG CTGGCTGGCA TAAATATCTC 1801 ACTCGCAATC AAATTCAGCC GATAGCGGAA CGGGAAGGCG ACTGGAGTGC 
1851 CATGTCCGGT TTTCAACAAA CCATGCAAAT GCTGAATGAG GGCATCGTTC 1901 CCACTGCGAT GCTGGTTGCC AACGATCAGA TGGCGCTGGG CGCAATGCGC 1951 GCCATTACCG AGTCCGGGCT GCGCGTTGGT GCGGACATCT CGGTAGTGGG 2001 ATACGACGAT ACCGAAGACA GCTCATGTTA TATCCCGCCG TTAACCACCA 2051 TCAAACAGGA TTTTCGCCTG CTGGGGCAAA CCAGCGTGGA CCGCTTGCTG 2101 CAACTCTCTC AGGGCCAGGC GGTGAAGGGC AATCAGCTGT TGCCCGTCTC 2151 ACTGGTGAAA AGAAAAACCA CCCTGGCGCC CAATACGCAA ACCGCCTCTC 2201 CCCGCGCGTT GGCCGATTCA TTAATGCAGC TGGCACGACA GGTTTCCCGA 2251 CTGGAAAGCG GGCAGTGAGC GCAACGCAAT TAATGTAAGT TAGCTCACTC 2301 ATTAGGCACC GGGATCTCGA CCGATGCCCT TGAGAGCCTT CAACCCAGTC 2351 AGCTCCTTCC GGTGGGCGCG GGGCATGACT ATCGTCGCCG CACTTATGAC 2401 TGTCTTCTTT ATCATGCAAC TCGTAGGACA GGTGCCGGCA GCGCTCTGGG 2451 TCATTTTCGG CGAGGACCGC TTTCGCTGGA GCGCGACGAT GATCGGCCTG 2501 TCGCTTGCGG TATTCGGAAT CTTGCACGCC CTCGCTCAAG CCTTCGTCAC 2551 TGGTCCCGCC ACCAAACGTT TCGGCGAGAA GCAGGCCATT ATCGCCGGCA 2601 TGGCGGCCCC ACGGGTGCGC ATGATCGTGC TCCTGTCGTT GAGGACCCGG 2651 CTAGGCTGGC GGGGTTGCCT TACTGGTTAG CAGAATGAAT CACCGATACG 2701 CGAGCGAACG TGAAGCGACT GCTGCTGCAA AACGTCTGCG ACCTGAGCAA 2751 CAACATGAAT GGTCTTCGGT TTCCGTGTTT CGTAAAGTCT GGAAACGCGG 2801 AAGTCAGCGC CCTGCACCAT TATGTTCCGG ATCTGCATCG CAGGATGCTG 2851 CTGGCTACCC TGTGGAACAC CTACATCTGT ATTAACGAAG CGCTGGCATT 2901 GACCCTGAGT GATTTTTCTC TGGTCCCGCC GCATCCATAC CGCCAGTTGT 2951 TTACCCTCAC AACGTTCCAG TAACCGGGCA TGTTCATCAT CAGTAACCCG 3001 TATCGTGAGC ATCCTCTCTC GTTTCATCGG TATCATTACC CCCATGAACA 3051 GAAATCCCCC TTACACGGAG GCATCAGTGA CCAAACAGGA AAAAACCGCC 3101 CTTAACATGG CCCGCTTTAT CAGAAGCCAG ACATTAACGC TTCTGGAGAA 3151 ACTCAACGAG CTGGACGCGG ATGAACAGGC AGACATCTGT GAATCGCTTC 3201 ACGACCACGC TGATGAGCTT TACCGCAGCT GCCTCGCGCG TTTCGGTGAT 3251 GACGGTGAAA ACCTCTGACA CATGCAGCTC CCGGAGACGG TCACAGCTTG 3301 TCTGTAAGCG GATGCCGGGA GCAGACAAGC CCGTCAGGGC GCGTCAGCGG 3351 GTGTTGGCGG GTGTCGGGGC GCAGCCATGA CCCAGTCACG TAGCGATAGC 3401 GGAGTGTATA CTGGCTTAAC TATGCGGCAT CAGAGCAGAT TGTACTGAGA 3451 GTGCACCATA TATGCGGTGT GAAATACCGC ACAGATGCGT AAGGAGAAAA 3501 TACCGCATCA GGCGCTCTTC CGCTTCCTCG CTCACTGACT CGCTGCGCTC 3551 GGTCGTTCGG CTGCGGCGAG CGGTATCAGC TCACTCAAAG GCGGTAATAC 3601 GGTTATCCAC AGAATCAGGGGATAACGCAG GAAAGAACATGTGAGCAAAA 3651 GGCCAGCAAA AGGCCAGGAA CCGTAAAAAG GCCGCGTTGC TGGCGTTTTT 3701 CCATAGGCTC CGCCCCCCTG ACGAGCATCA CAAAAATCGA CGCTCAAGTC 3751 AGAGGTGGCG AAACCCGACA GGACTATAAA GATACCAGGC GTTTCCCCCT 3801 GGAAGCTCCC TCGTGCGCTC TCCTGTTCCG ACCCTGCCGC TTACCGGATA 
3851 CCTGTCCGCC TTTCTCCCTT CGGGAAGCGT GGCGCTTTCT CATAGCTCAC 3901 GCTGTAGGTA TCTCAGTTCG GTGTAGGTCG TTCGCTCCAA GCTGGGCTGT 3951 GTGCACGAAC CCCCCGTTCA GCCCGACCGC TGCGCCTTAT CCGGTAACTA 4001 TCGTCTTGAG TCCAACCCGG TAAGACACGA CTTATCGCCA CTGGCAGCAG 4051 CCACTGGTAA CAGGATTAGC AGAGCGAGGT ATGTAGGCGG TGCTACAGAG 4101 TTCTTGAAGT GGTGGCCTAA CTACGGCTAC ACTAGAAGGA CAGTATTTGG 4151 TATCTGCGCT CTGCTGAAGC CAGTTACCTT CGGAAAAAGA GTTGGTAGCT 4201 CTTGATCCGG CAAACAAACC ACCGCTGGTA GCGGTGGTTT TTTTGTTTGC 4251 AAGCAGCAGA TTACGCGCAG AAAAAAAGGA TCTCAAGAAG ATCCTTTGAT 4301 CTTTTCTACG GGGTCTGACG CTCAGTGGAA CGAAAACTCA CGTTAAGGGA 4351 TTTTGGTCAT GAACAATAAA ACTGTCTGCT TACATAAACA GTAATACAAG 4401 GGGTGTTATG AGCCATATTC AACGGGAAAC GTCTTGCTCT AGGCCGCGAT 4451 TAAATTCCAA CATGGATGCT GATTTATATG GGTATAAATG GGCTCGCGAT 4501 AATGTCGGGC AATCAGGTGC GACAATCTAT CGATTGTATG GGAAGCCCGA 4551 TGCGCCAGAG TTGTTTCTGA AACATGGCAA AGGTAGCGTT GCCAATGATG 4601 TTACAGATGA GATGGTCAGA CTAAACTGGC TGACGGAATT TATGCCTCTT 4651 CCGACCATCA AGCATTTTAT CCGTACTCCT GATGATGCAT GGTTACTCAC 4701 CACTGCGATC CCCGGGAAAA CAGCATTCCA GGTATTAGAA GAATATCCTG 4751 ATTCAGGTGA AAATATTGTT GATGCGCTGG CAGTGTTCCT GCGCCGGTTG 4801 CATTCGATTC CTGTTTGTAA TTGTCCTTTT AACAGCGATC GCGTATTTCG 4851 TCTCGCTCAG GCGCAATCAC GAATGAATAA CGGTTTGGTT GATGCGAGTG 4901 ATTTTGATGA CGAGCGTAAT GGCTGGCCTG TTGAACAAGT CTGGAAAGAA 4951 ATGCATAAAC TTTTGCCATT CTCACCGGAT TCAGTCGTCA CTCATGGTGA 5001 TTTCTCACTT GATAACCTTA TTTTTGACGA GGGGAAATTA ATAGGTTGTA 5051 TTGATGTTGG ACGAGTCGGA ATCGCAGACC GATACCAGGA TCTTGCCATC 5101 CTATGGAACT GCCTCGGTGA GTTTTCTCCT TCATTACAGA AACGGCTTTT 5151 TCAAAAATAT GGTATTGATA ATCCTGATAT GAATAAATTG CAGTTTCATT 5201 TGATGCTCGA TGAGTTTTTC TAAGAATTAA TTCATGAGCG GATACATATT 5251 TGAATGTATT TAGAAAAATA AACAAATAGG GGTTCCGCGC ACATTTCCCC 5301 GAAAAGTGCC ACCTGAAATT GTAAACGTTA ATATTTTGTT AAAATTCGCG 5351 TTAAATTTTT GTTAAATCAG CTCATTTTTT AACCAATAGG CCGAAATCGG 5401 CAAAATCCCT TATAAATCAA AAGAATAGAC CGAGATAGGG TTGAGTGTTG 5451 TTCCAGTTTG GAACAAGAGT CCACTATTAA AGAACGTGGA CTCCAACGTC 5501 AAAGGGCGAA AAACCGTCTA TCAGGGCGAT GGCCCACTAC GTGAACCATC 5551 ACCCTAATCA AGTTTTTTGG GGTCGAGGTG CCGTAAAGCA CTAAATCGGA 5601 ACCCTAAAGG GAGCCCCCGA TTTAGAGCTT GACGGGGAAA GCCGGCGAAC 5651GTGGCGAGAAAGGAAGGGAAGAAAGCGAAA GGAGCGGGCG CTAGGGCGCT 5701 GCAAGTGTA GCGGTCACGC TGCGCGTAAC CACCACACCC GCCGCGCTTA 5751 ATGCGCCGCT ACAGGGCGCG TCCCATTCGC CA 


\section{APPENDIX C}

\section{General information}

Table C1 and C2 list all the primers designed for wild type cytochrome P450cam and sperm whale myoglobin and their mutants in this dissertation study. 


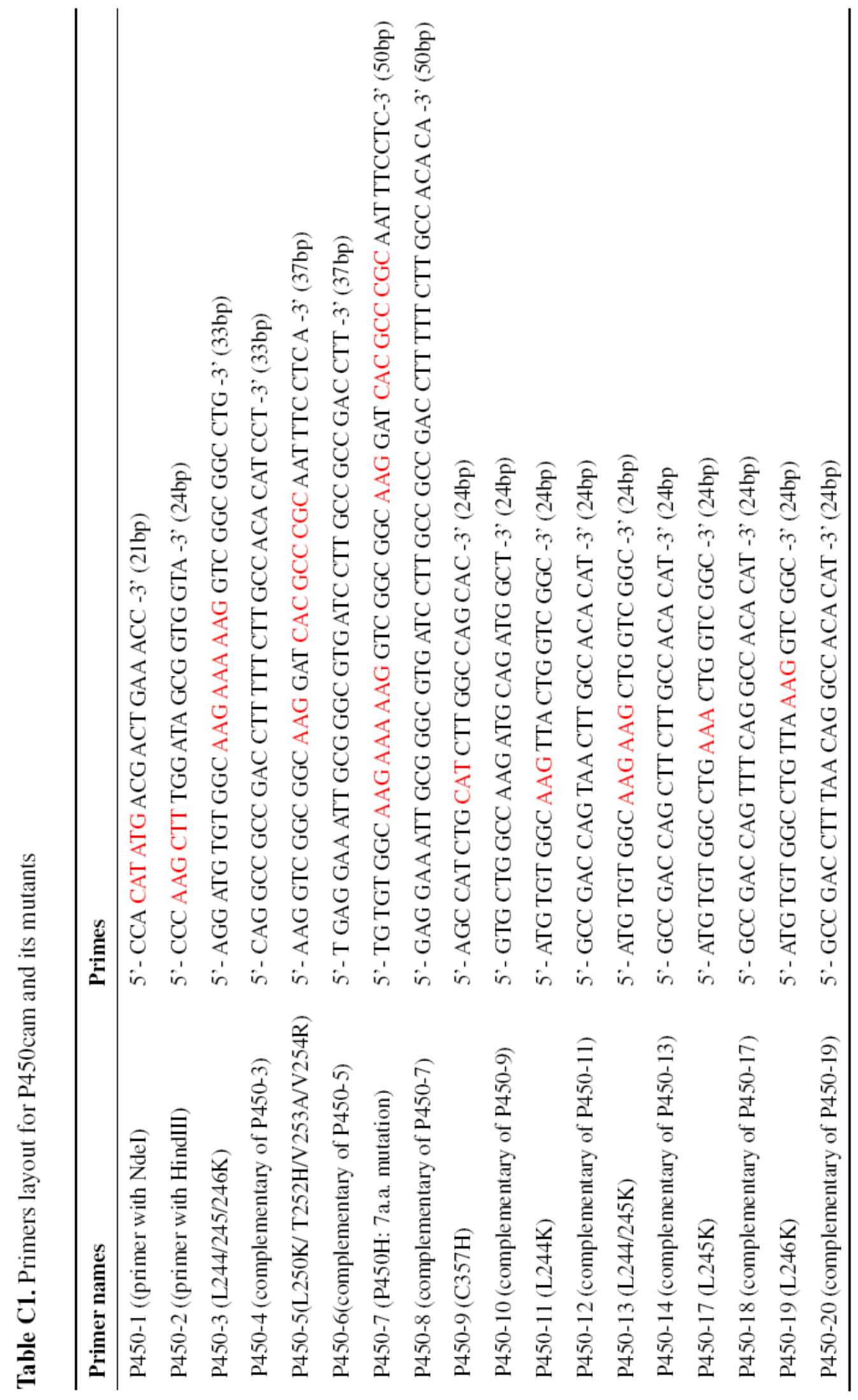




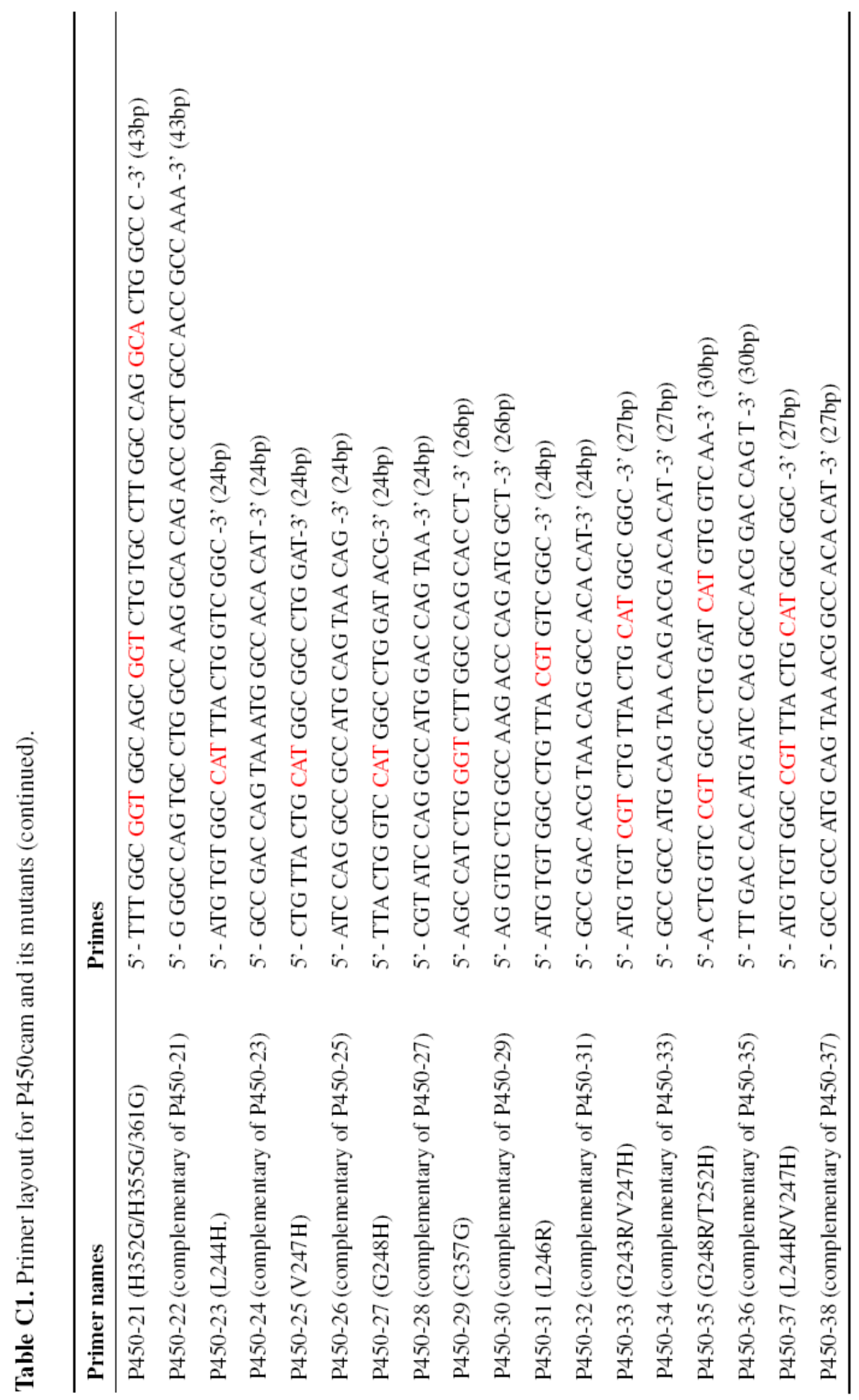




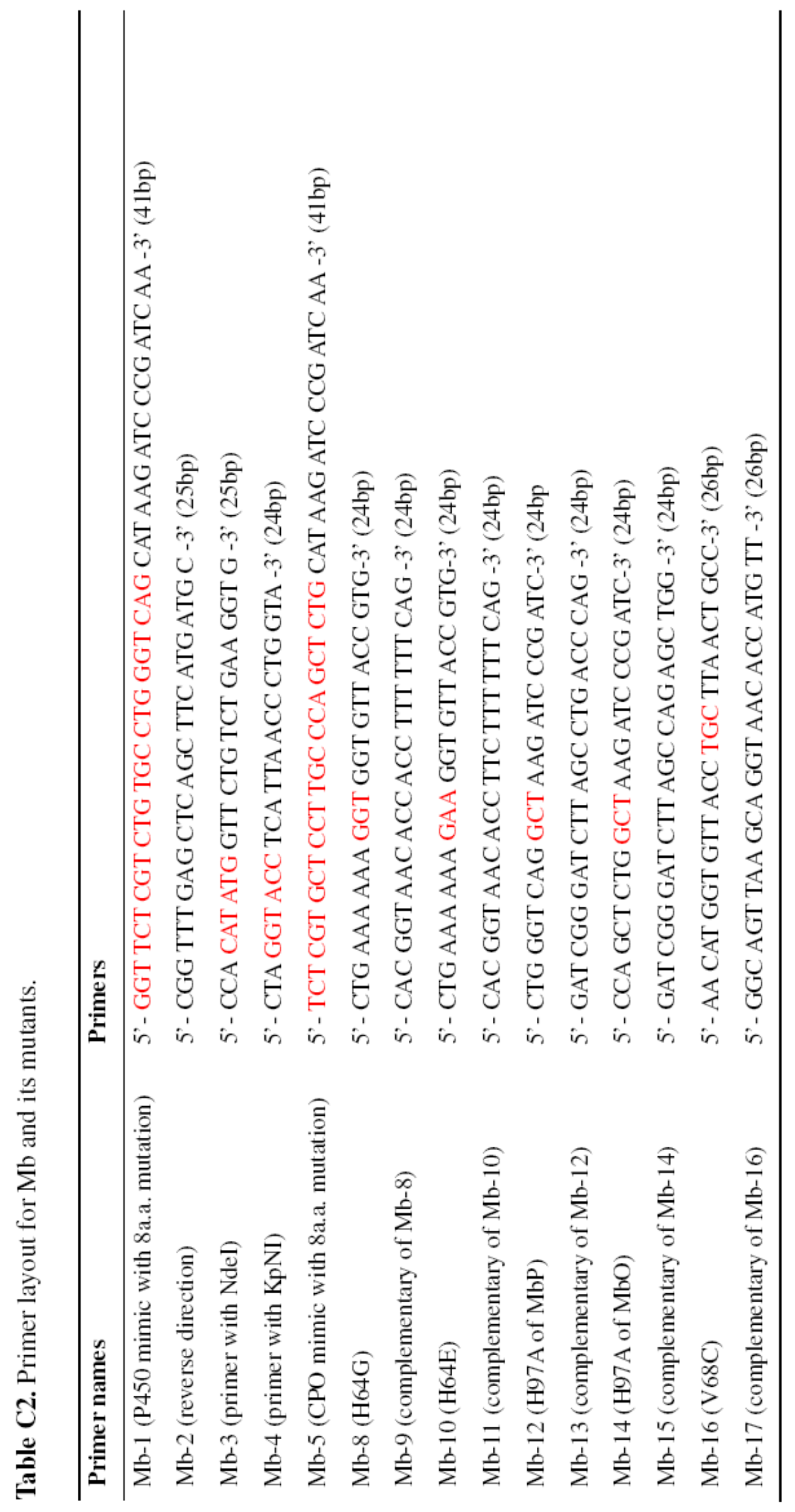


VITA

\section{HUI TIAN}

May 19, 1981

1999-2003

2010
Born, Hebei, China

Bachelor, Chemical Engineering and Technology

Hebei University of Technology, China

Doctoral Candidate in Chemistry

Florida International University

Miami, Florida

\section{PUBLICATIONS AND PRESENTATIONS}

Tian, Hui; Wang, Zhonghua; Terentis, Andrew; Wang, Xiaotang. Relative stability of iron-sulfur bond vs iron-nitrogen bond in heme thiolate protein: Evidence from an engineered cytochrome P450cam with a novel heme iron ligand set. Abstracts of Papers, 237th ACS National Meeting, Washington D.C., United States, August 17-22, 2009

Tian, Hui; Wang, Zhonghua; Terentis, Andrew; Wang, Xiaotang. Single amino acid switch turns P450cam from a monooxygenase to an efficient peroxidase. Abstracts of Papers, 237th ACS National Meeting, Salt Lake City, UT, United States, March 22-26, 2009

Tian, Hui; Jiang, Yucheng; Wang, Xiaotang. Engineering P450 into P420 with catalytic activities. Abstracts of Papers, 235th ACS National Meeting, New Orleans, LA, United States, April 6-10, 2008

Mulchandani, Avani; Tian, Hui; Wang, Xiaotang; Li, Chenzhong. Nanomaterials functionalized device for redox studies of cytochrome P450cam L246K. Abstracts of Papers, Biotech 2008, Miami, FL, United States, April 24, 2008

Wang, Xiaotang; Wang, Zheng; Jiang, Yucheng; Tian, Hui. Catalytic roles of Phe103 at the substrate-binding pocket of chloroperoxidase: Remote control of heme chemistry in heme-thiolate proteins. Abstracts of Papers, 234th ACS National Meeting, Boston, MA, United States, August 19-23, 2007 
Wang, Xiaotang; Jiang, Yucheng; Zhang, Chengxiao; Wang, Zheng; Tian, Hui. Structure of the green prosthetic group in chloroperoxidase following mechanism-based modification with terminal alkenes. Abstracts of Papers, 232nd ACS National Meeting, San Francisco, CA, United States, Sept. 10-14, 2006 